Supporting information for

\title{
An Air-Stable Organic Radical from a Controllable Photoinduced Domino Reaction of a Hexa-aryl Substituted Anthracene
}

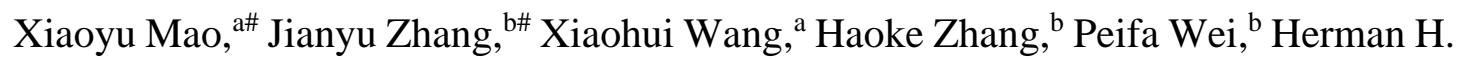
Y. Sung, ${ }^{\mathrm{b}}$ Ian D. Williams, ${ }^{\mathrm{b}}$ Xing Feng, ${ }^{* a}$ Xin-Long Ni, ${ }^{\mathrm{c}}$ Carl Redshaw, ${ }^{\mathrm{d}}$ Mark R. J. Elsegood, ${ }^{\mathrm{e}}$ Jacky W. Y. Lam, ${ }^{\mathrm{b}}$ Ben Zhong Tang, ${ }^{* \mathrm{~b}}$

${ }^{\mathrm{a}}$ Guangdong Provincial Key Laboratory of Functional Soft Condensed Matter, School of Material and Energy, Guangdong University of Technology, Guangzhou 510006, P. R. China. Email: hyxhn@sina.com (X.Feng)

${ }^{b}$ Department of Chemistry, The Hong Kong Branch of Chinese National Engineering Research Center for Tissue Restoration and Reconstruction, Institute for Advanced Study, Department of Chemical and Biological Engineering, Division of Life Science, State Key Laboratory of Molecular Neuroscience, The Hong Kong University of Science and Technology, Clear Water Bay, Kowloon, Hong Kong, China. Email: tangbenz@ust.hk (B.Z. Tang)

${ }^{\mathrm{c} C o l l e g e}$ of Chemistry and Chemical Engineering, Key Laboratory of the Assembly and Application of Organic Functional Molecules of Hunan Province, Hunan Normal University, Changsha 410081, P.R. China. E-mail: longni333@163.com (X.L. Ni)

${ }^{\mathrm{d} D e p a r t m e n t}$ of Chemistry, University of Hull, Cottingham Road, Hull, Yorkshire HU6 7RX, UK.

${ }^{\mathrm{e}}$ Chemistry Department, Loughborough University, Loughborough LE11 3TU, UK. 


\section{Table of Content}

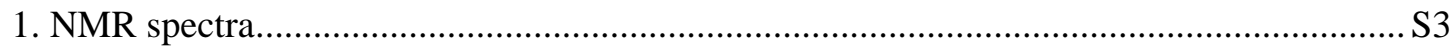

1.1 The NMR spectrum of synthesized compounds ......................................................... 3

1.2 Irradiation Time-Dependent ${ }^{1}$ H NMR Spectrum................................................... 223

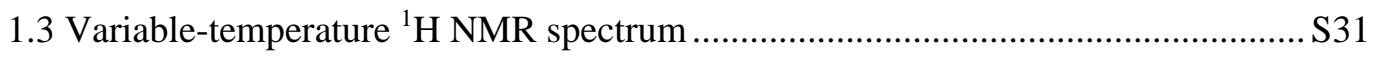

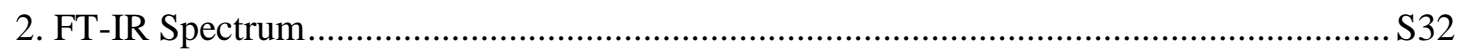

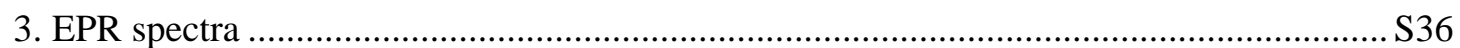

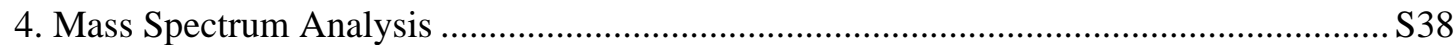

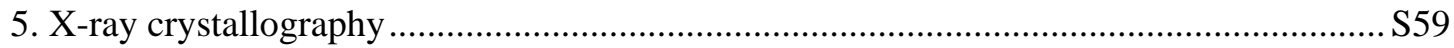

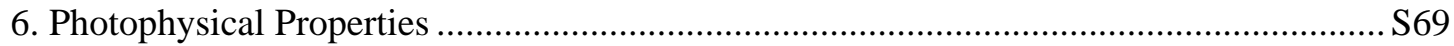

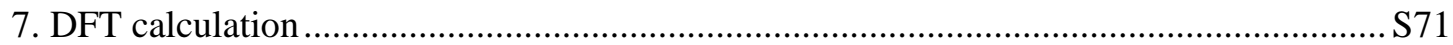




\section{NMR spectra}

\subsection{The NMR spectrum of synthesized compounds}

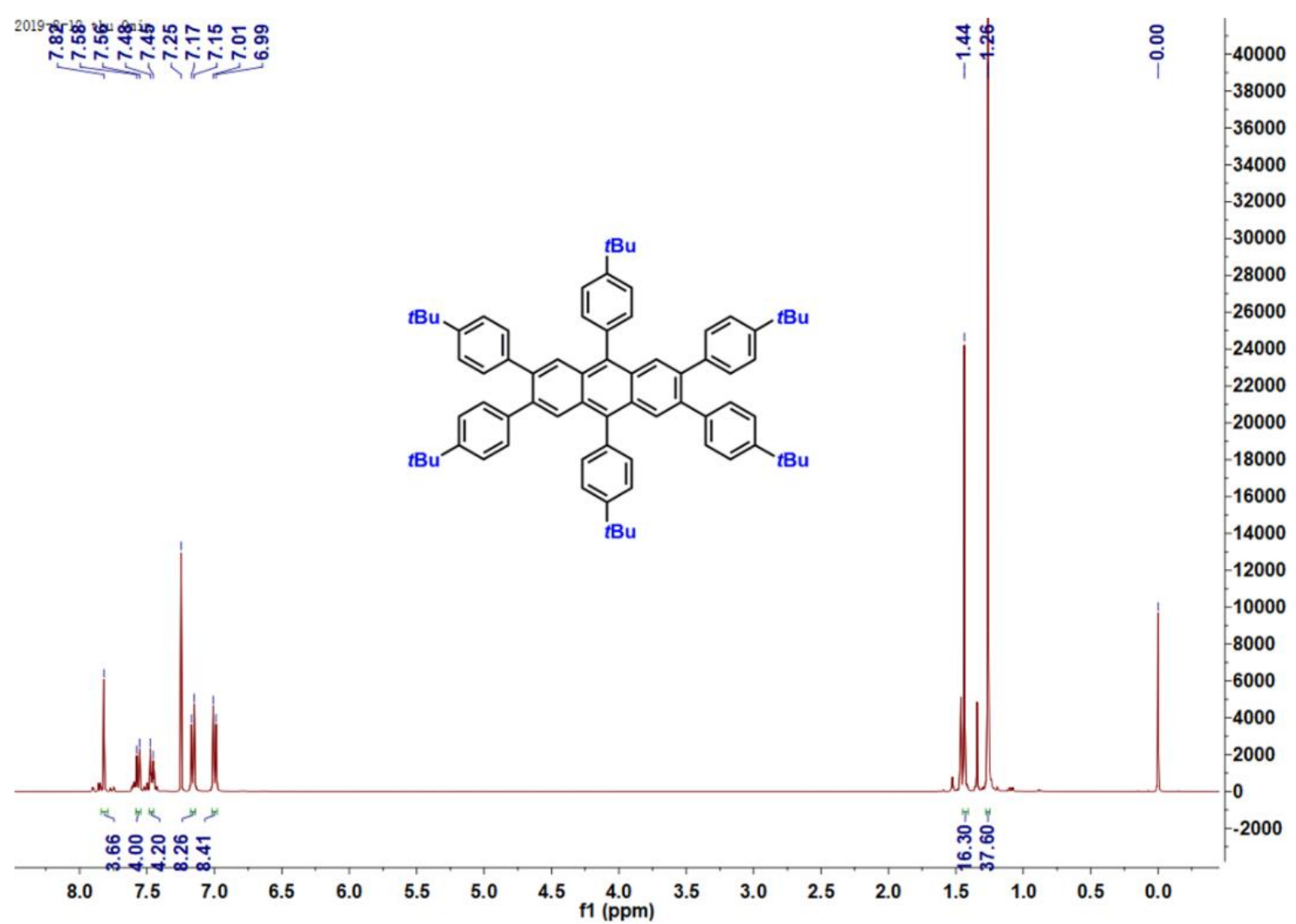

Figure S1 ${ }^{1} \mathrm{H}$ NMR spectrum $\left(400 \mathrm{MHz}, 293 \mathrm{~K},{ }^{*} \mathrm{CDCl}_{3}\right)$ for 2,3,6,7,9,10-hexa-( $p$-tert-butyl phenyl) anthracene $\mathbf{2 a}$ 


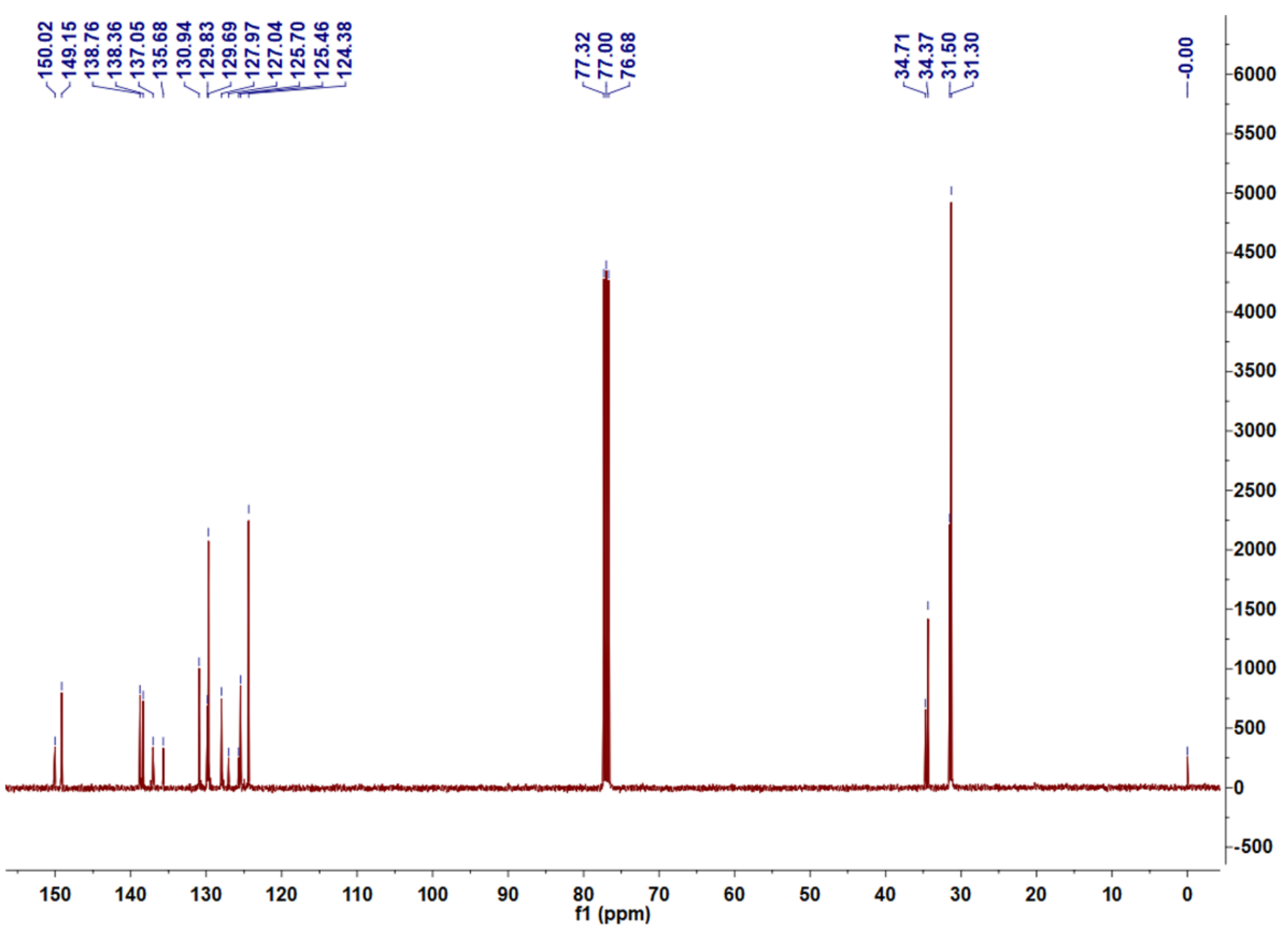

Figure S2 ${ }^{13} \mathrm{C}$ NMR spectrum $\left(100 \mathrm{MHz}, 293 \mathrm{~K},{ }^{*} \mathrm{CDCl}_{3}\right)$ for 2,3,6,7,9,10-hexa-(p-tert-butyl phenyl) anthracene 2a 


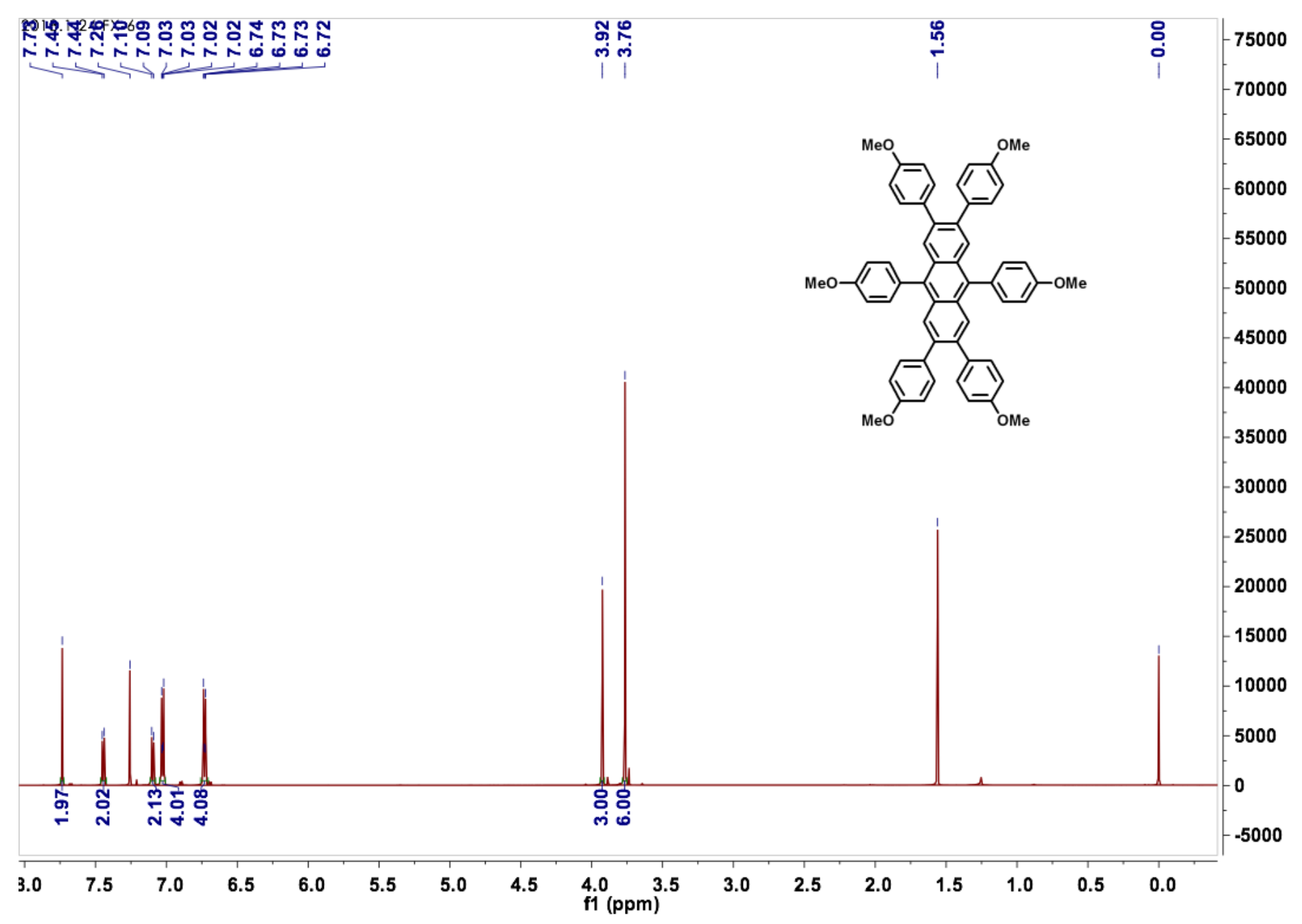

Figure $\mathbf{S 3}{ }^{1} \mathrm{H}$ NMR spectrum $\left(400 \mathrm{MHz}, 293 \mathrm{~K},{ }^{*} \mathrm{CDCl}_{3}\right)$ for 2,3,6,7,9,10-hexa-( $p$-methoxyl-phenyl) anthracene $\mathbf{2 b .}$ 


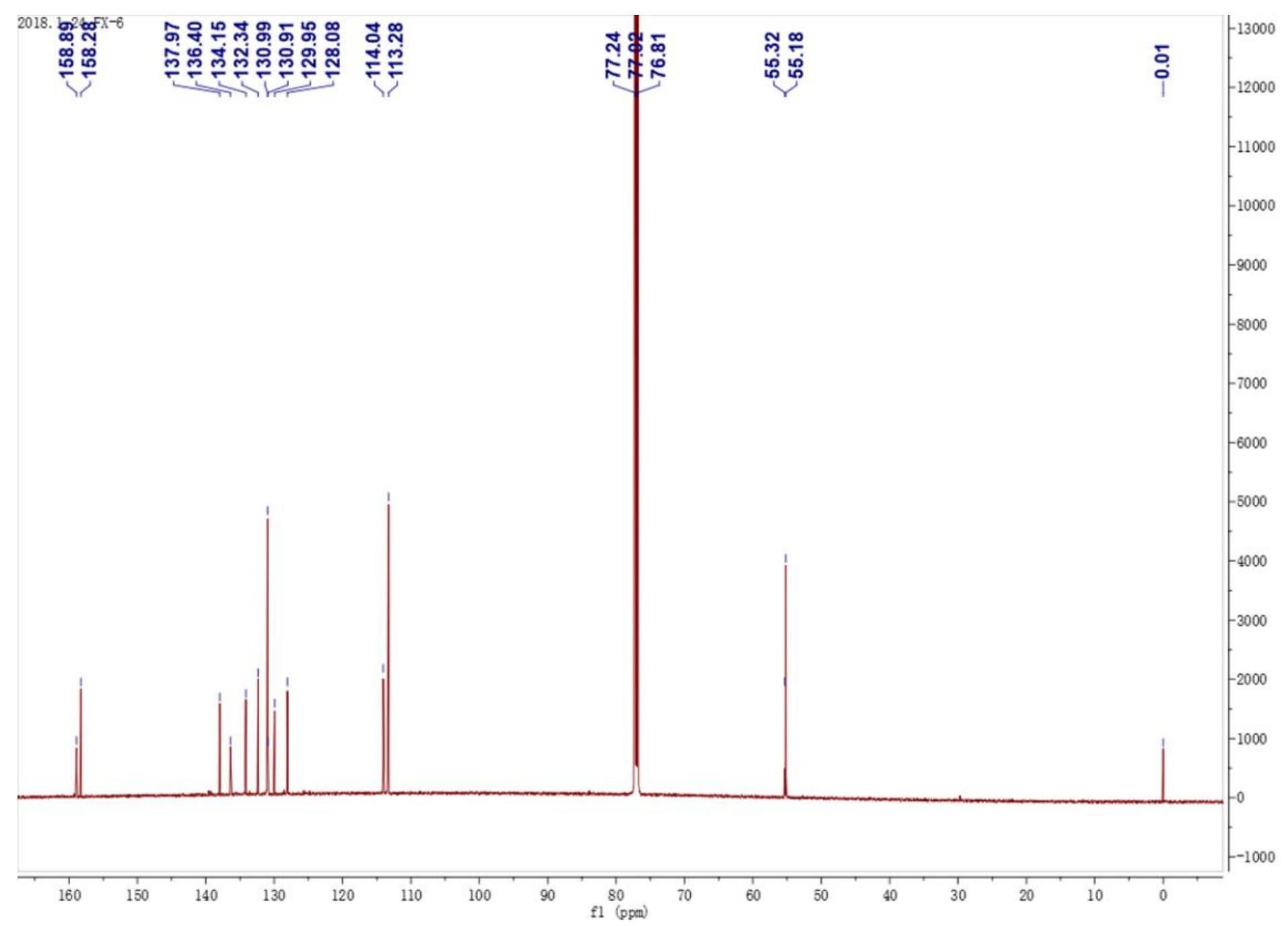

Figure $\mathbf{S 4}{ }^{13} \mathrm{C}$ NMR spectrum $\left(100 \mathrm{MHz}, 293 \mathrm{~K},{ }^{*} \mathrm{CDCl}_{3}\right)$ for 2,3,6,7,9,10-hexa-( $p$-methoxylphenyl) anthracene $\mathbf{2 b}$. 


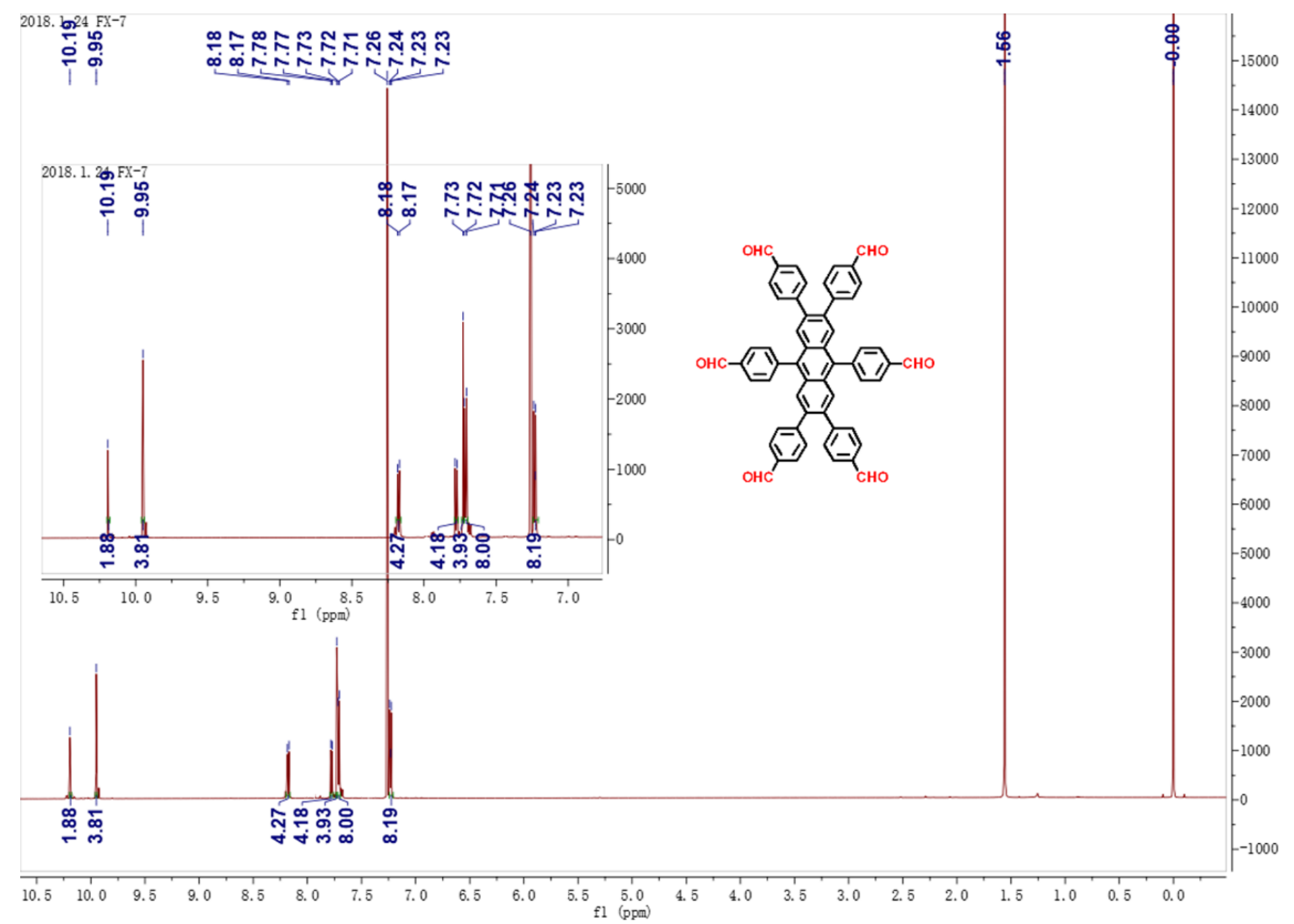

Figure S5 ${ }^{1} \mathrm{H}$ NMR spectrum $\left(400 \mathrm{MHz}, 293 \mathrm{~K},{ }^{*} \mathrm{CDCl}_{3}\right)$ for 2,3,6,7,9,10-hexa-( $p$-formyl-phenyl) anthracene $2 \mathbf{c}$. 


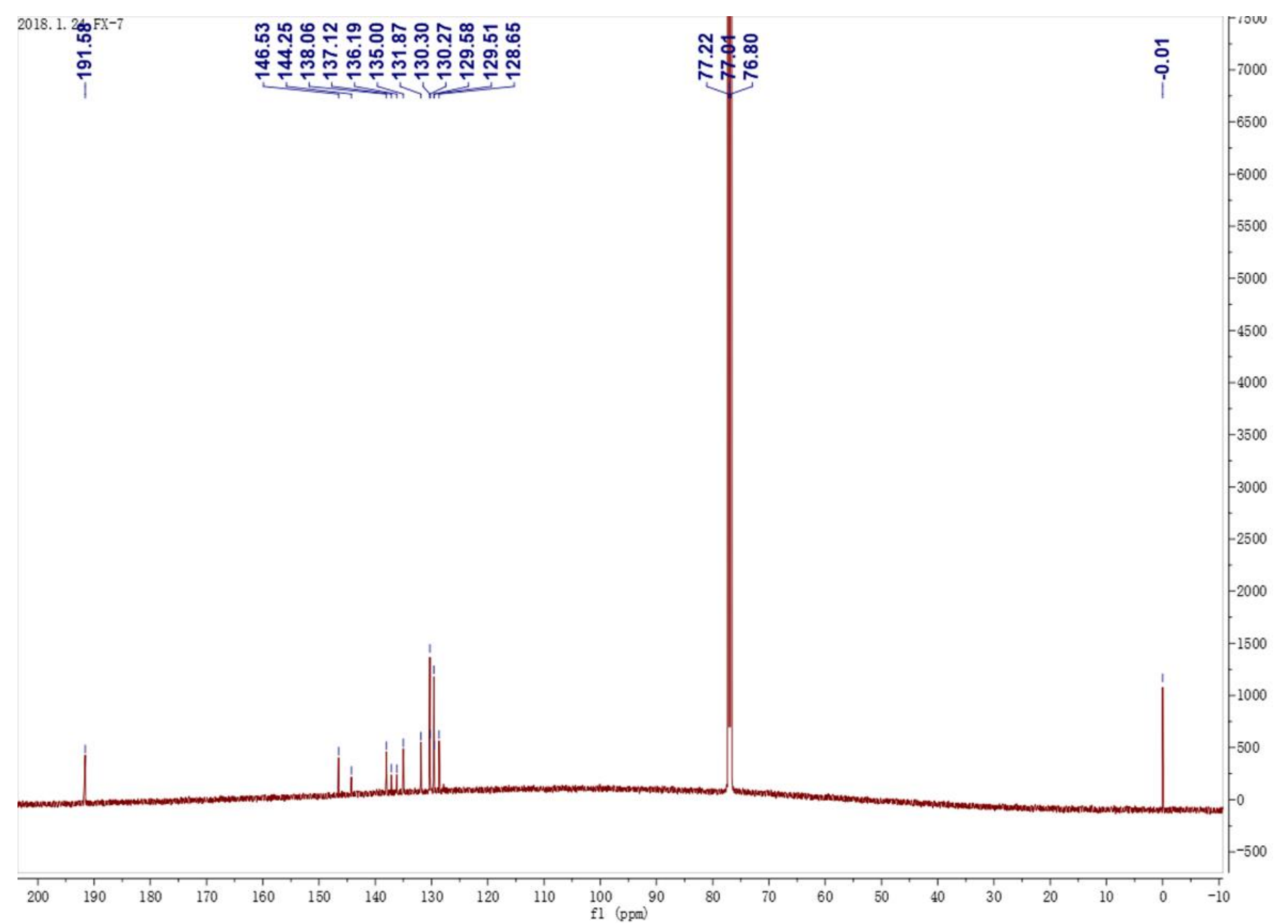

Figure S6 ${ }^{13} \mathrm{C}$ NMR spectrum $\left(100 \mathrm{MHz}, 293 \mathrm{~K},{ }^{*} \mathrm{CDCl}_{3}\right)$ for 2,3,6,7,9,10-hexa-( $p$-formylphenyl) anthracene $2 \mathrm{c}$. 


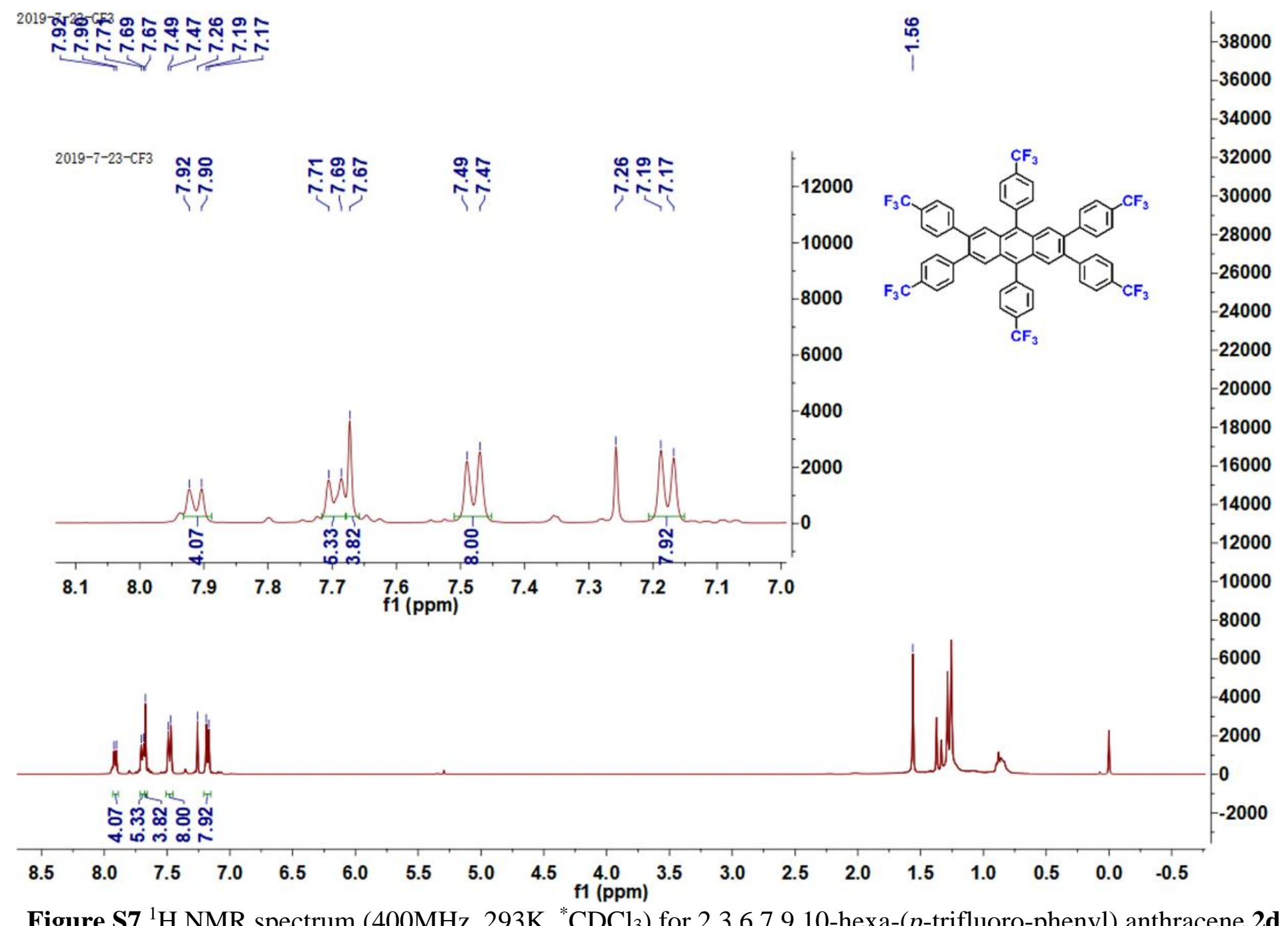




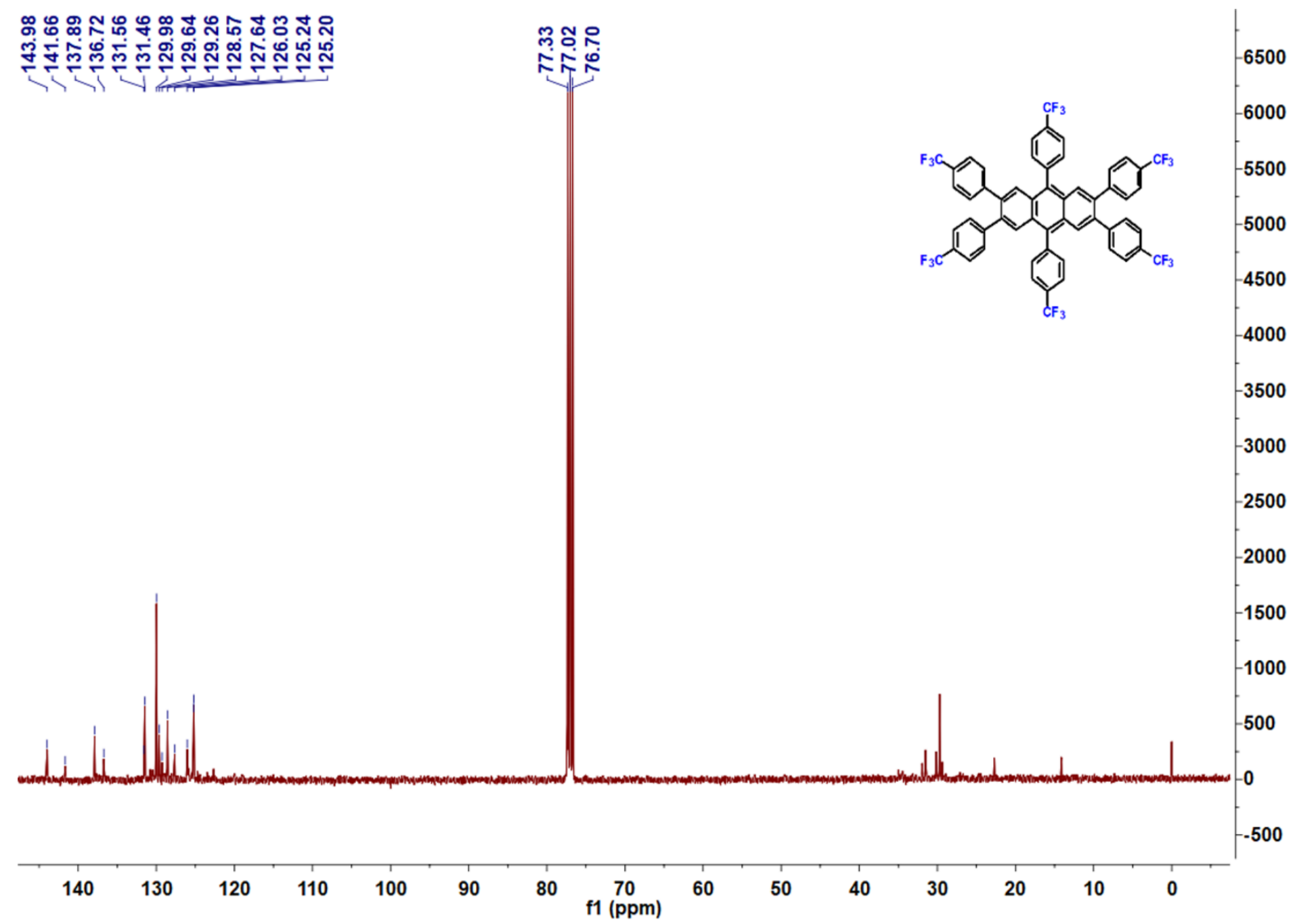

Figure S8 ${ }^{13} \mathrm{C}$ NMR spectrum $\left(100 \mathrm{MHz}, 293 \mathrm{~K},{ }^{*} \mathrm{CDCl}_{3}\right)$ for 2,3,6,7,9,10-hexa-( $p$-trifluoro-phenyl) anthracene $2 \mathbf{d}$. 


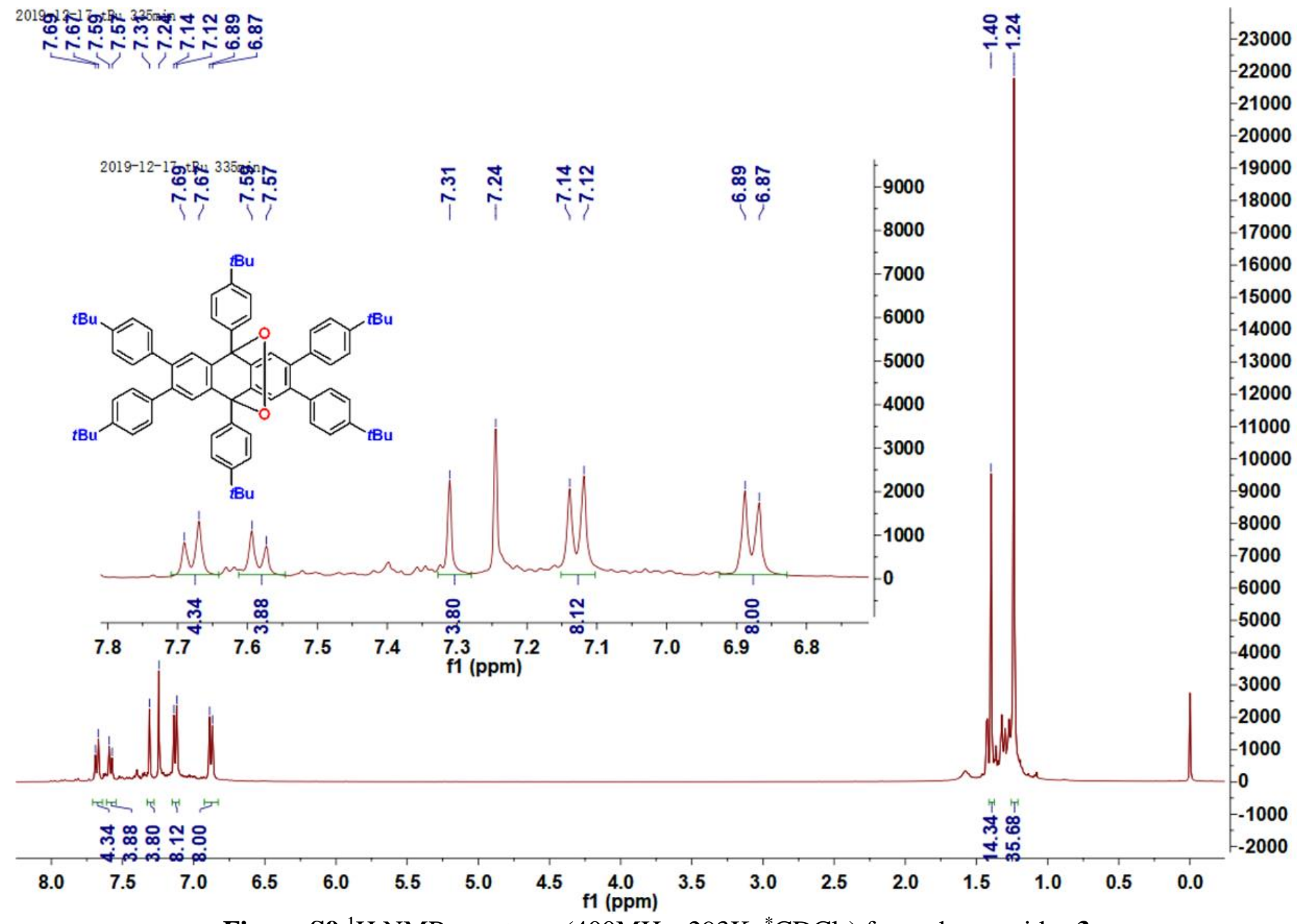

Figure S9 ${ }^{1} \mathrm{H}$ NMR spectrum $\left(400 \mathrm{MHz}, 293 \mathrm{~K},{ }^{*} \mathrm{CDCl}_{3}\right)$ for endoperoxides $3 \mathbf{a}$ 


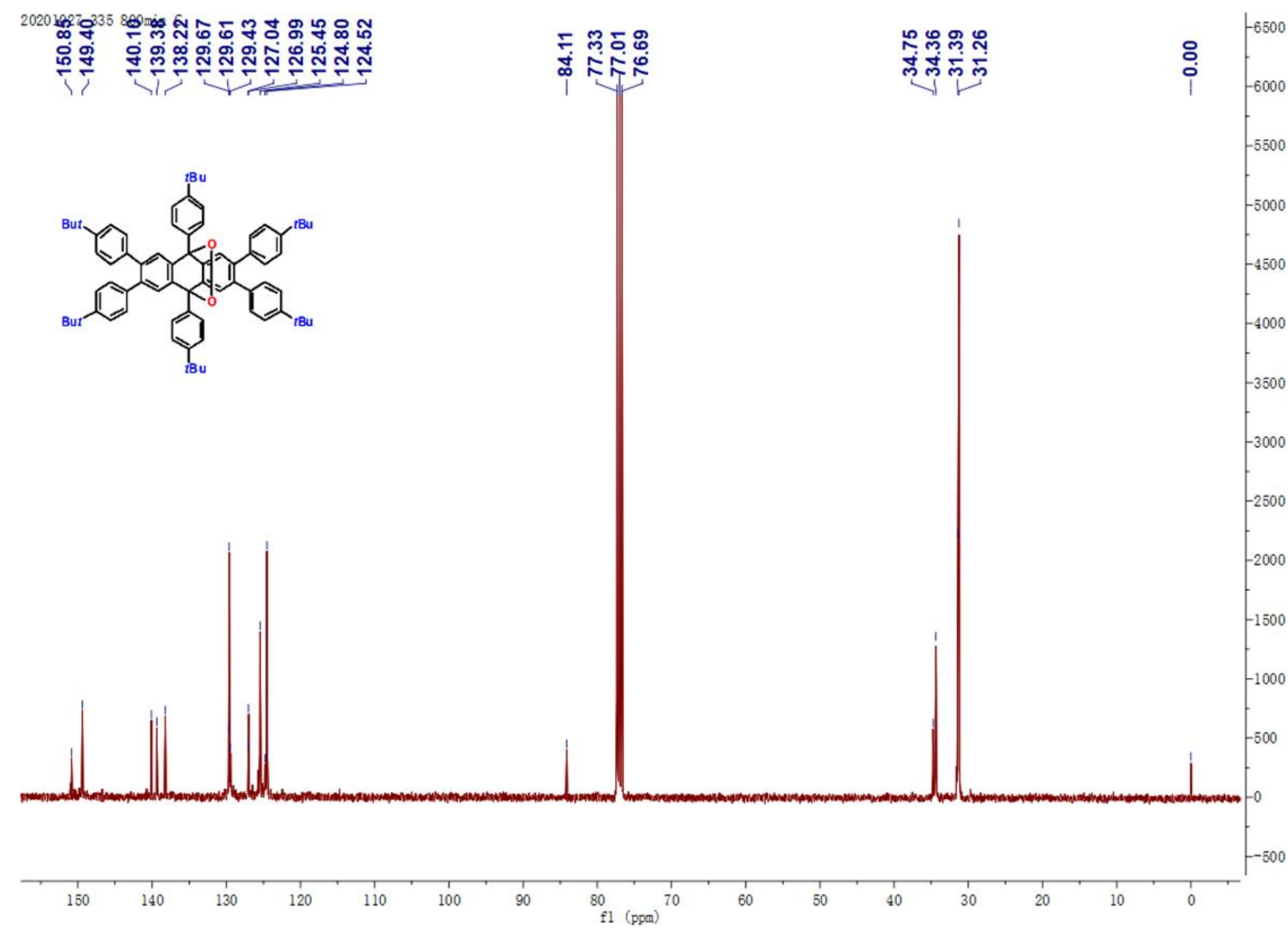

Figure $\mathbf{S 1 0}{ }^{13} \mathrm{C}$ NMR spectrum $\left(100 \mathrm{MHz}, 293 \mathrm{~K},{ }^{*} \mathrm{CDCl}_{3}\right)$ for endoperoxides $\mathbf{3 a}$ 


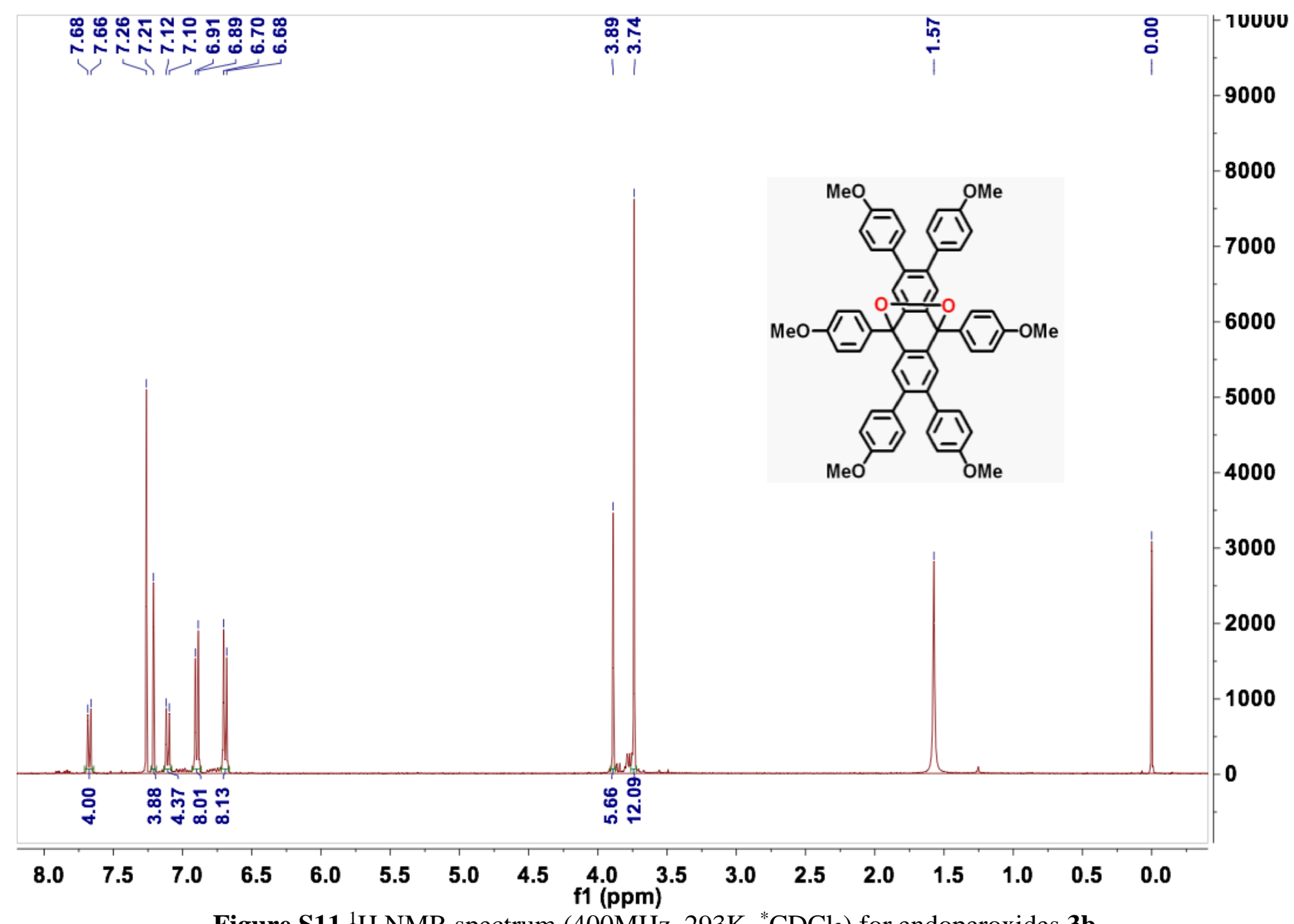

Figure $\mathbf{S 1 1}{ }^{1} \mathrm{H}$ NMR spectrum $\left(400 \mathrm{MHz}, 293 \mathrm{~K},{ }^{*} \mathrm{CDCl}_{3}\right)$ for endoperoxides $\mathbf{3 b}$ 


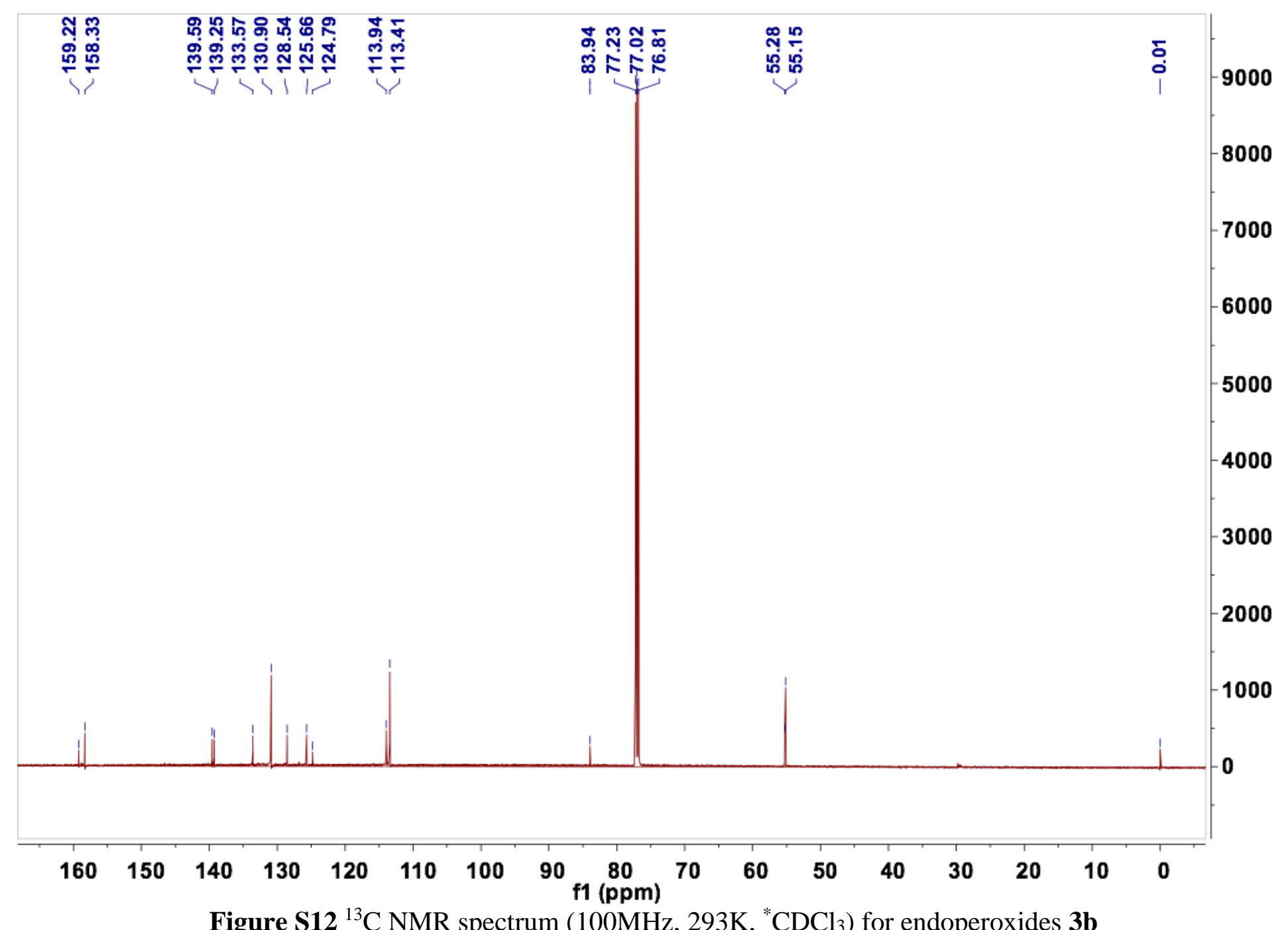

Figure S12 ${ }^{13} \mathrm{C}$ NMR spectrum $\left(100 \mathrm{MHz}, 293 \mathrm{~K},{ }^{*} \mathrm{CDCl}_{3}\right)$ for endoperoxides $\mathbf{3 b}$ 


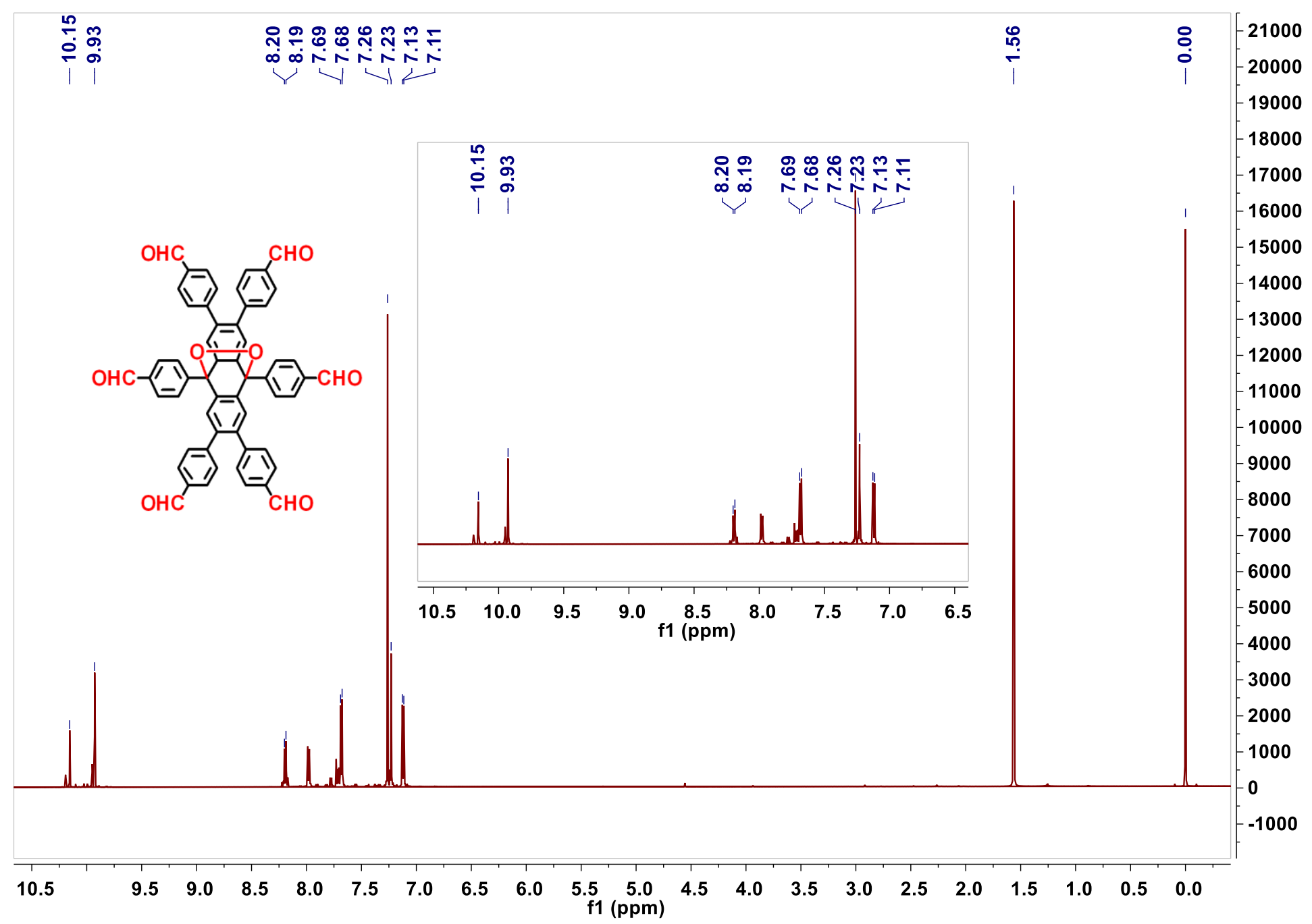

Figure $\mathbf{S 1 3}{ }^{1} \mathrm{H}$ NMR spectrum $\left(400 \mathrm{MHz}, 293 \mathrm{~K},{ }^{*} \mathrm{CDCl}_{3}\right)$ for endoperoxides 3c. 


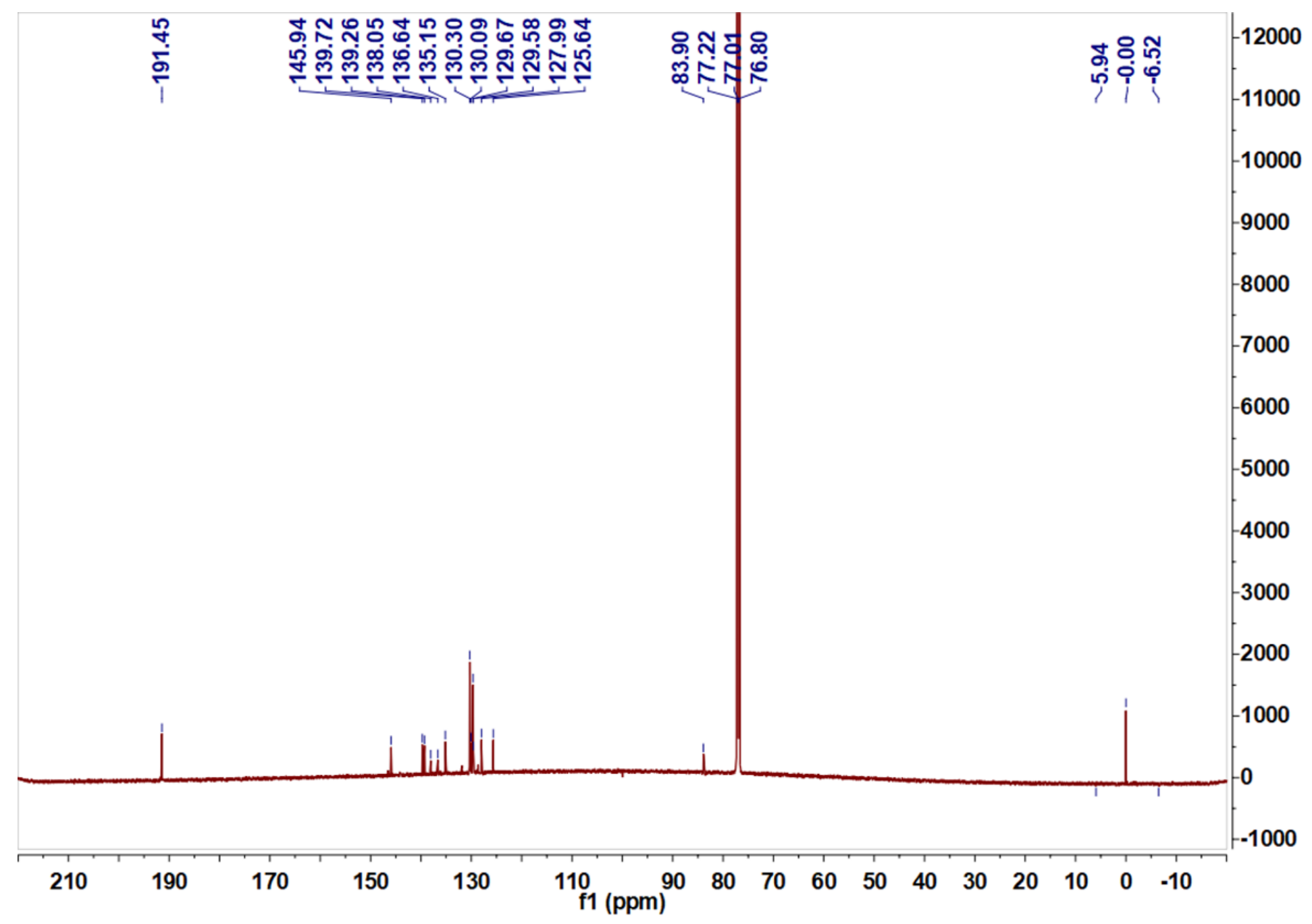

Figure $\mathbf{S 1 4}{ }^{13} \mathrm{C}$ NMR spectrum $\left(100 \mathrm{MHz}, 293 \mathrm{~K},{ }^{*} \mathrm{CDCl}_{3}\right)$ for endoperoxides $3 \mathbf{c}$. 


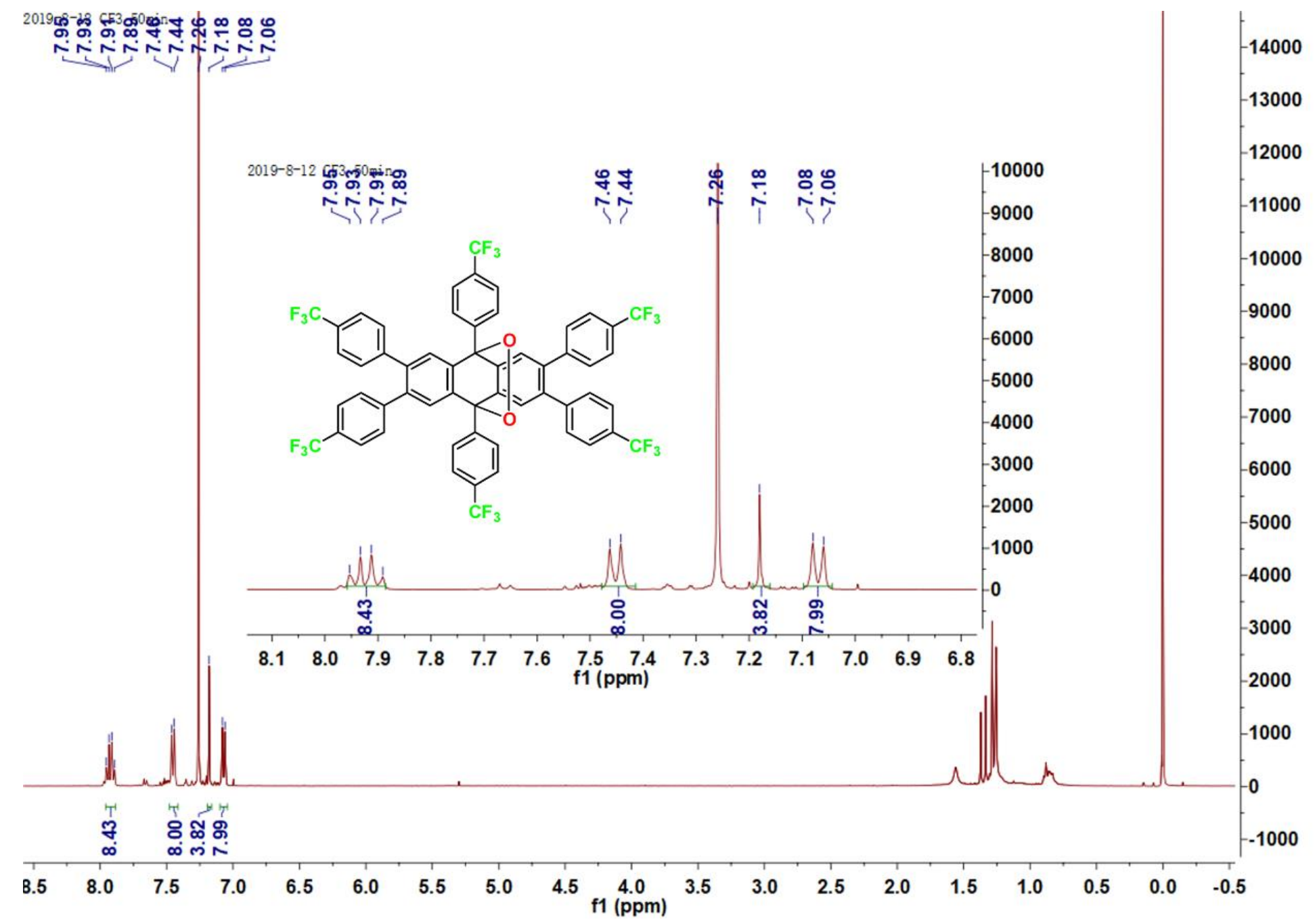

Figure $\mathbf{S 1 5}{ }^{1} \mathrm{H}$ NMR spectrum $\left(400 \mathrm{MHz}, 293 \mathrm{~K},{ }^{*} \mathrm{CDCl}_{3}\right)$ for endoperoxides $\mathbf{3 d}$ 


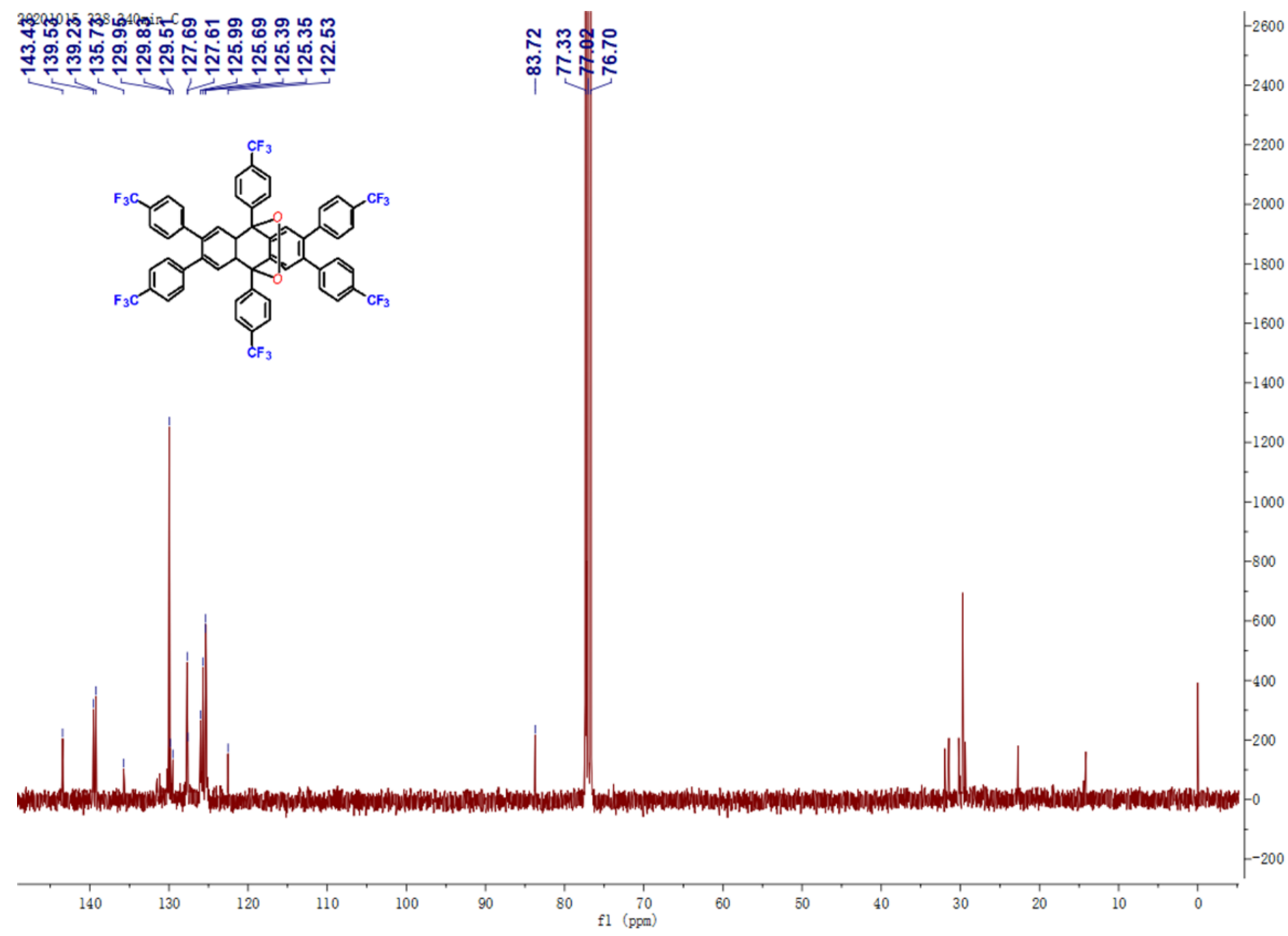

Figure S16 ${ }^{13} \mathrm{C}$ NMR spectrum $\left(100 \mathrm{MHz}, 293 \mathrm{~K},{ }^{*} \mathrm{CDCl}_{3}\right)$ for endoperoxides $\mathbf{3 d}$ 


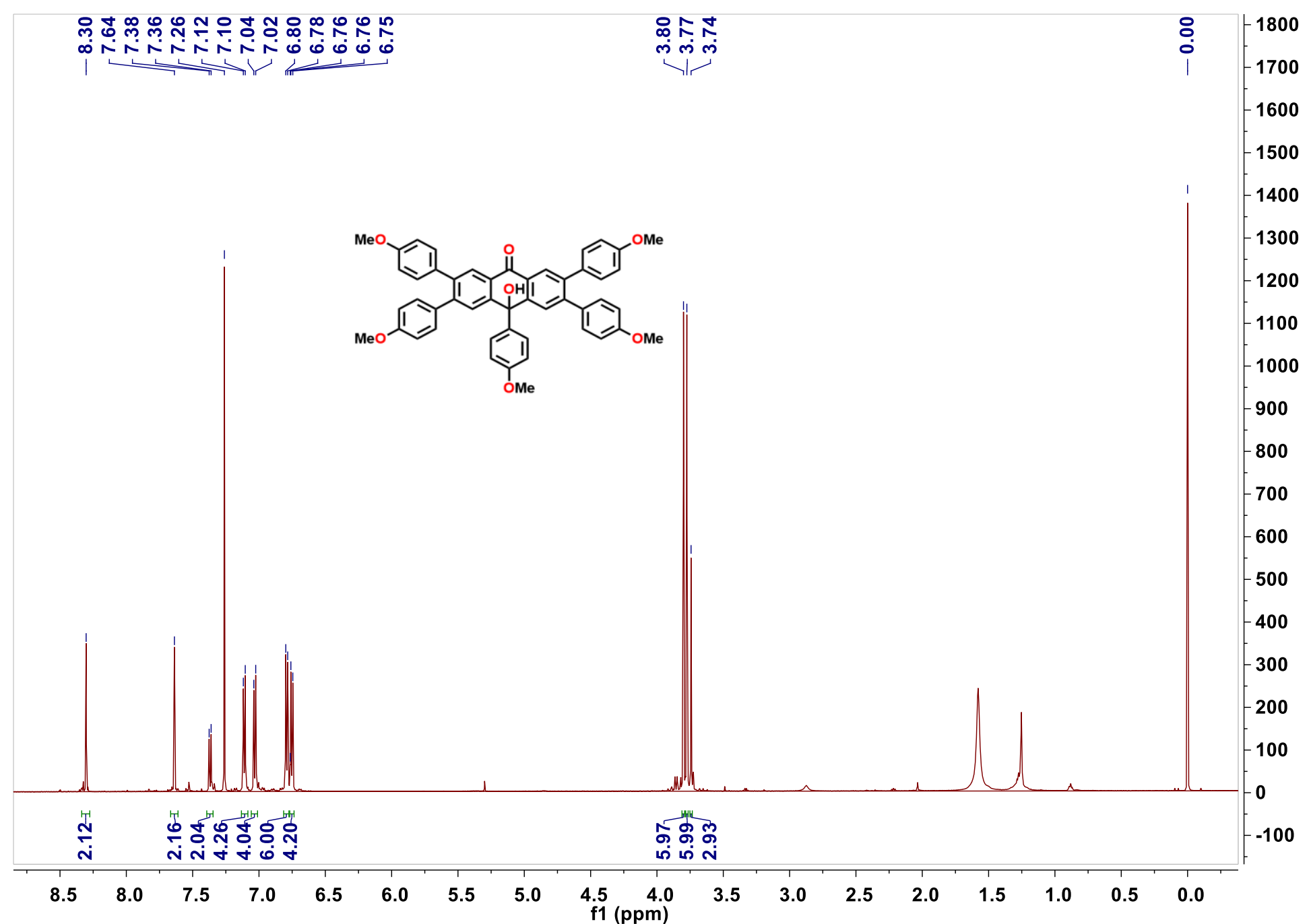

Figure $\mathbf{S 1 7}{ }^{1} \mathrm{H}$ NMR spectrum $\left(400 \mathrm{MHz}, 293 \mathrm{~K},{ }^{*} \mathrm{CDCl}_{3}\right)$ for radical $\mathbf{4 b}$ 


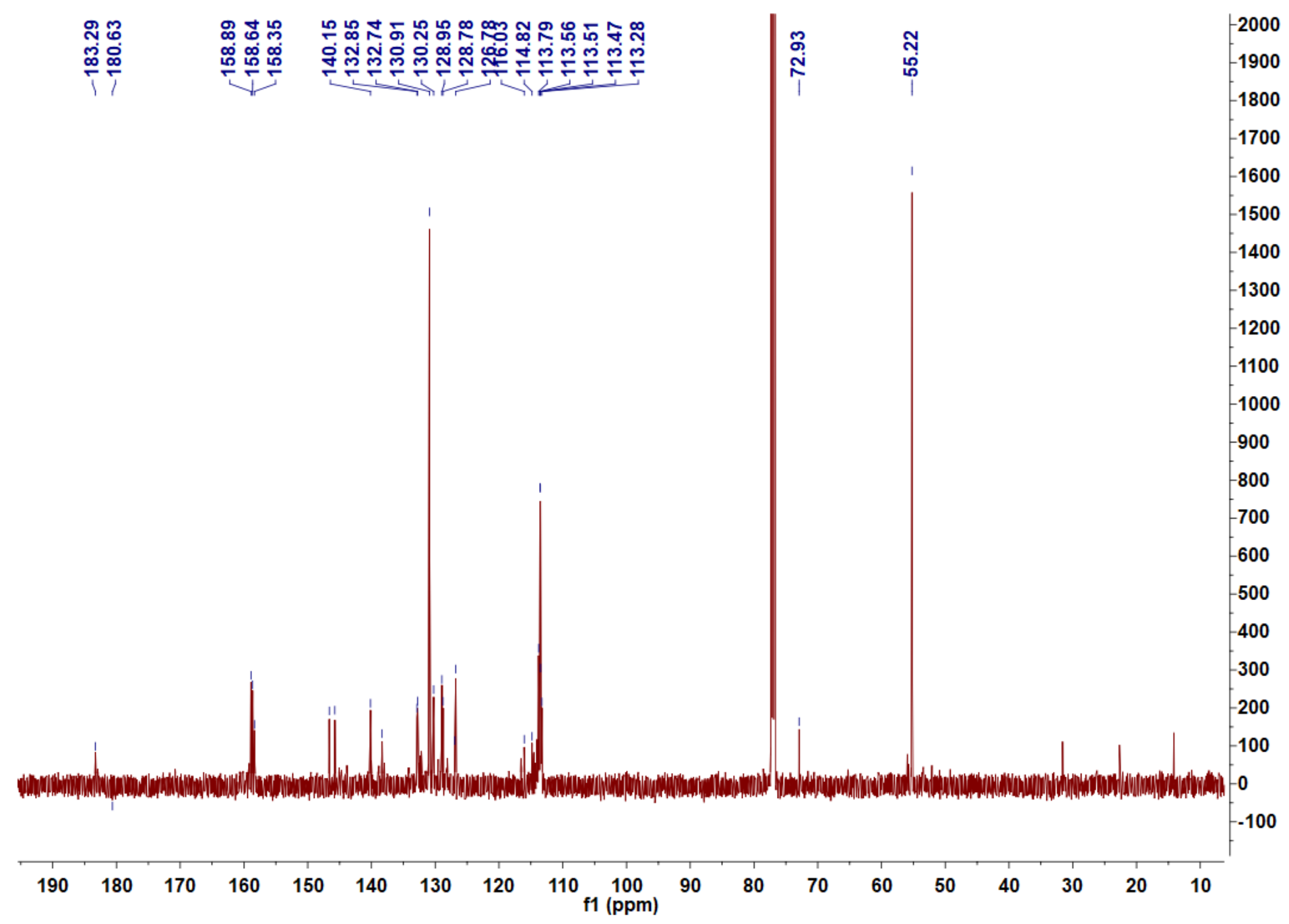

Figure S18 ${ }^{13} \mathrm{C}$ NMR spectrum $\left(100 \mathrm{MHz}, 293 \mathrm{~K},{ }^{*} \mathrm{CDCl}_{3}\right)$ for radical $\mathbf{4 b}$ 


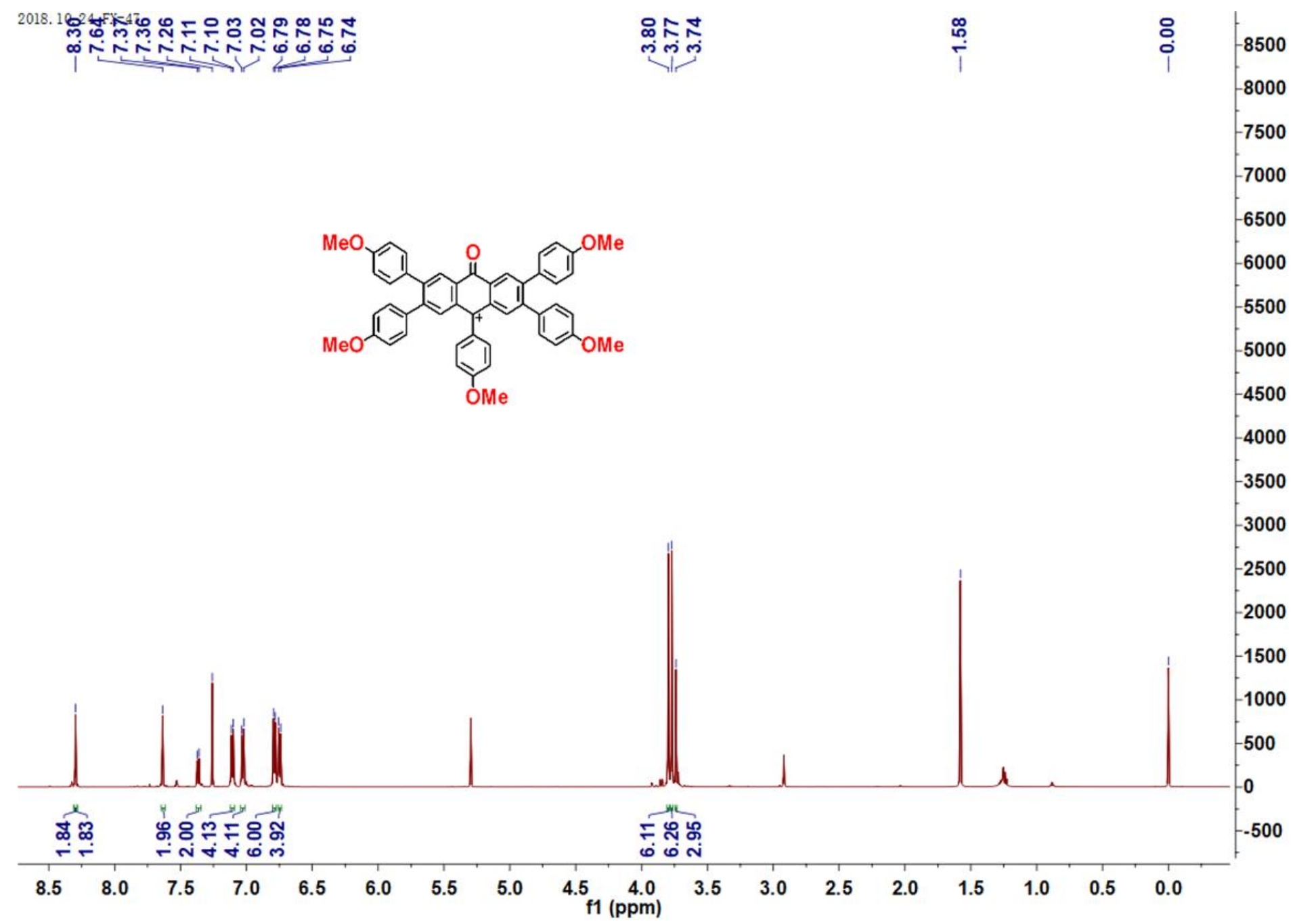

Figure S19 ${ }^{1} \mathrm{H}$ NMR spectrum $\left(400 \mathrm{MHz}, 293 \mathrm{~K},{ }^{*} \mathrm{CDCl}_{3}\right)$ for radical $\mathbf{5 b}$ 


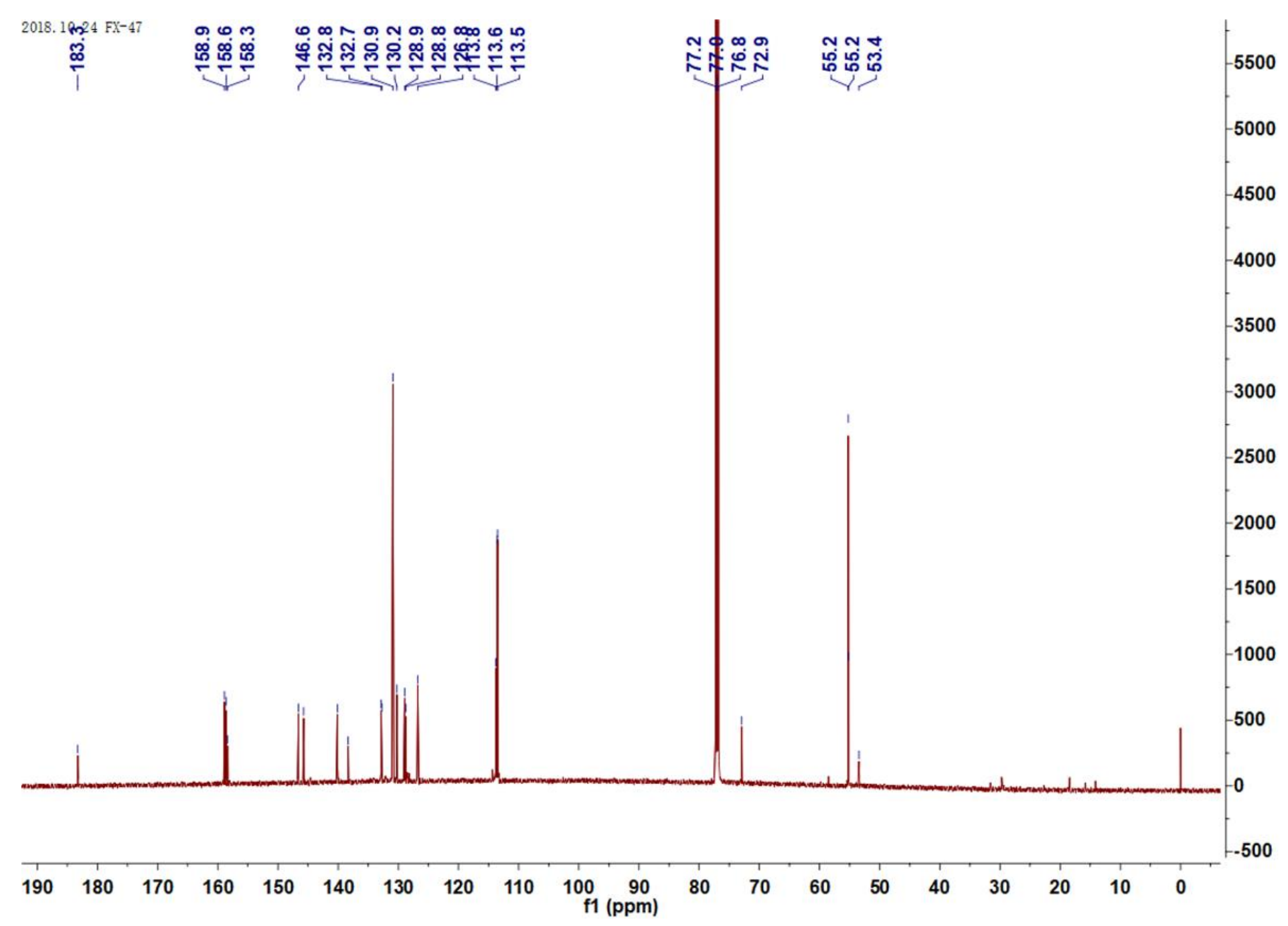

Figure S20 ${ }^{13} \mathrm{C}$ NMR spectrum $\left(100 \mathrm{MHz}, 293 \mathrm{~K},{ }^{*} \mathrm{CDCl}_{3}\right)$ for radical $\mathbf{5 b}$ 


\subsection{Irradiation Time-Dependent ${ }^{1} \mathrm{H}$ NMR Spectrum}

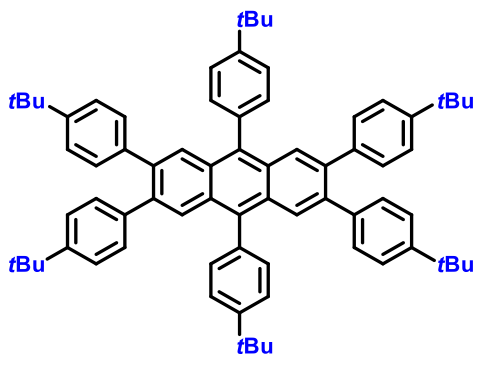

$2 \mathbf{a}$

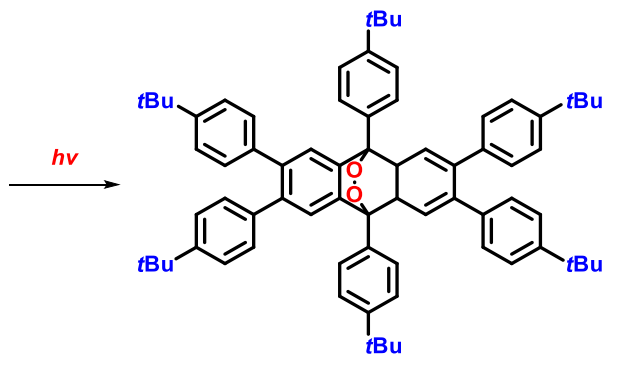

3a

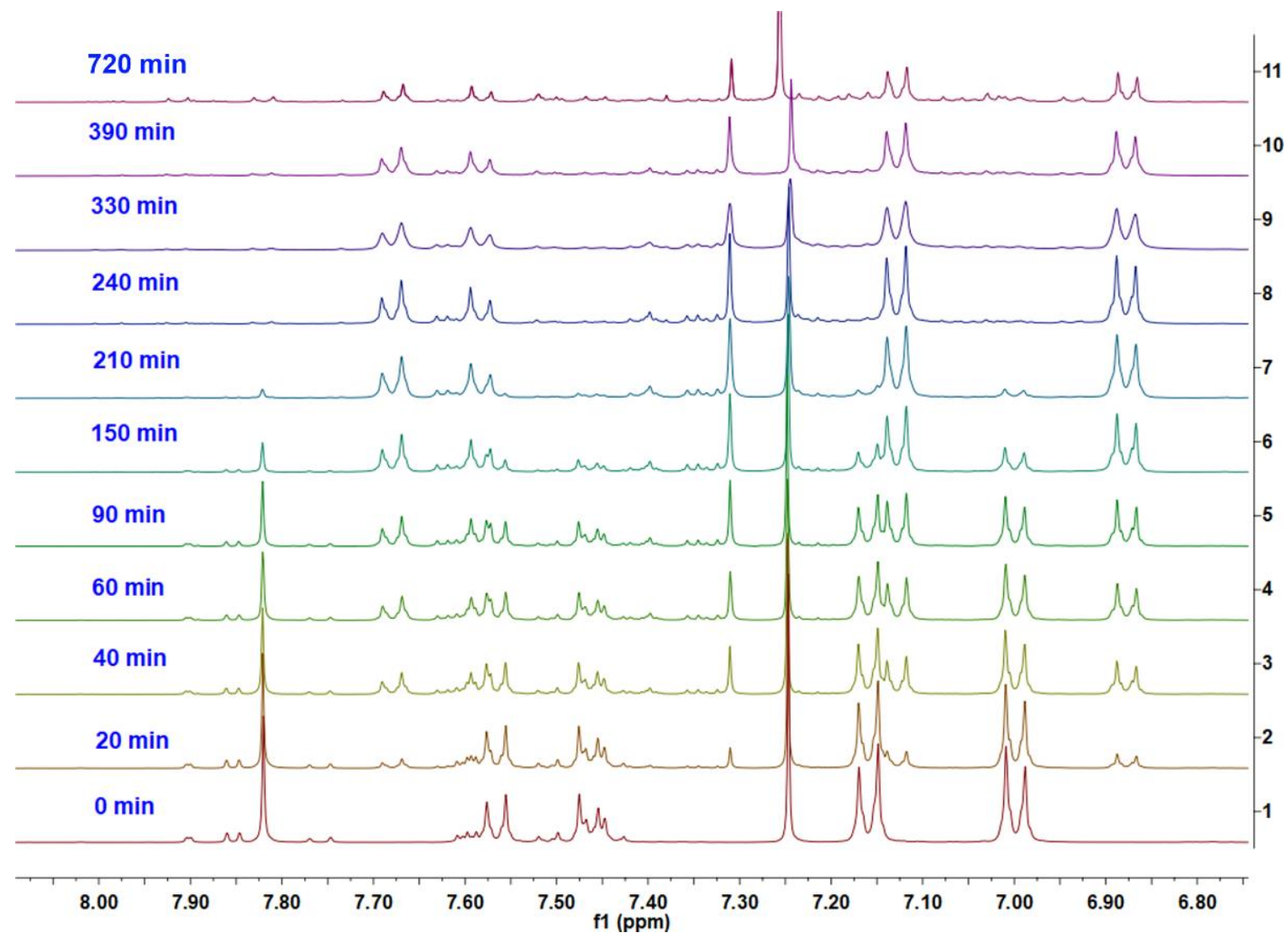

Figure S21 ${ }^{1} \mathrm{H}$ NMR spectrum $\left(400 \mathrm{MHz}, 293 \mathrm{~K},{ }^{*} \mathrm{CDCl}_{3}\right)$ of compound 2a to 3a under UV irradiation $\left(\lambda_{\mathrm{ex}}=365 \mathrm{~nm}\right)$ with different time. 


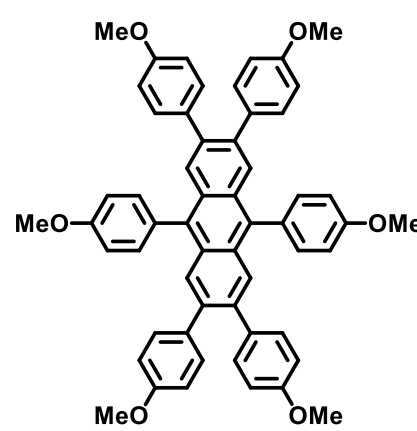

2b

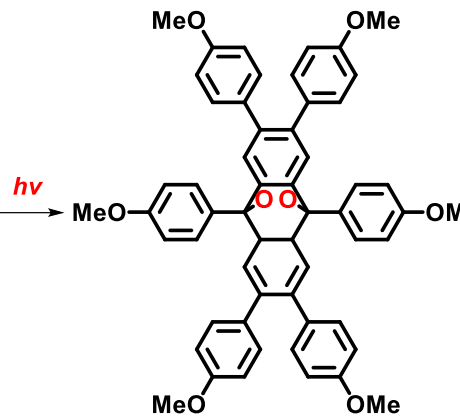

$3 \mathbf{b}$

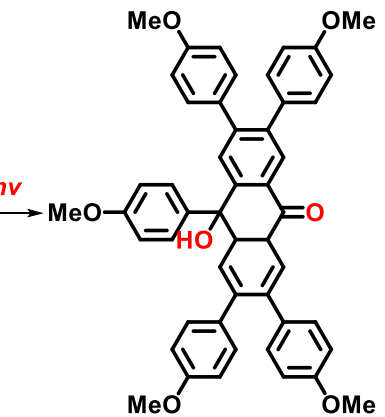

$4 b$

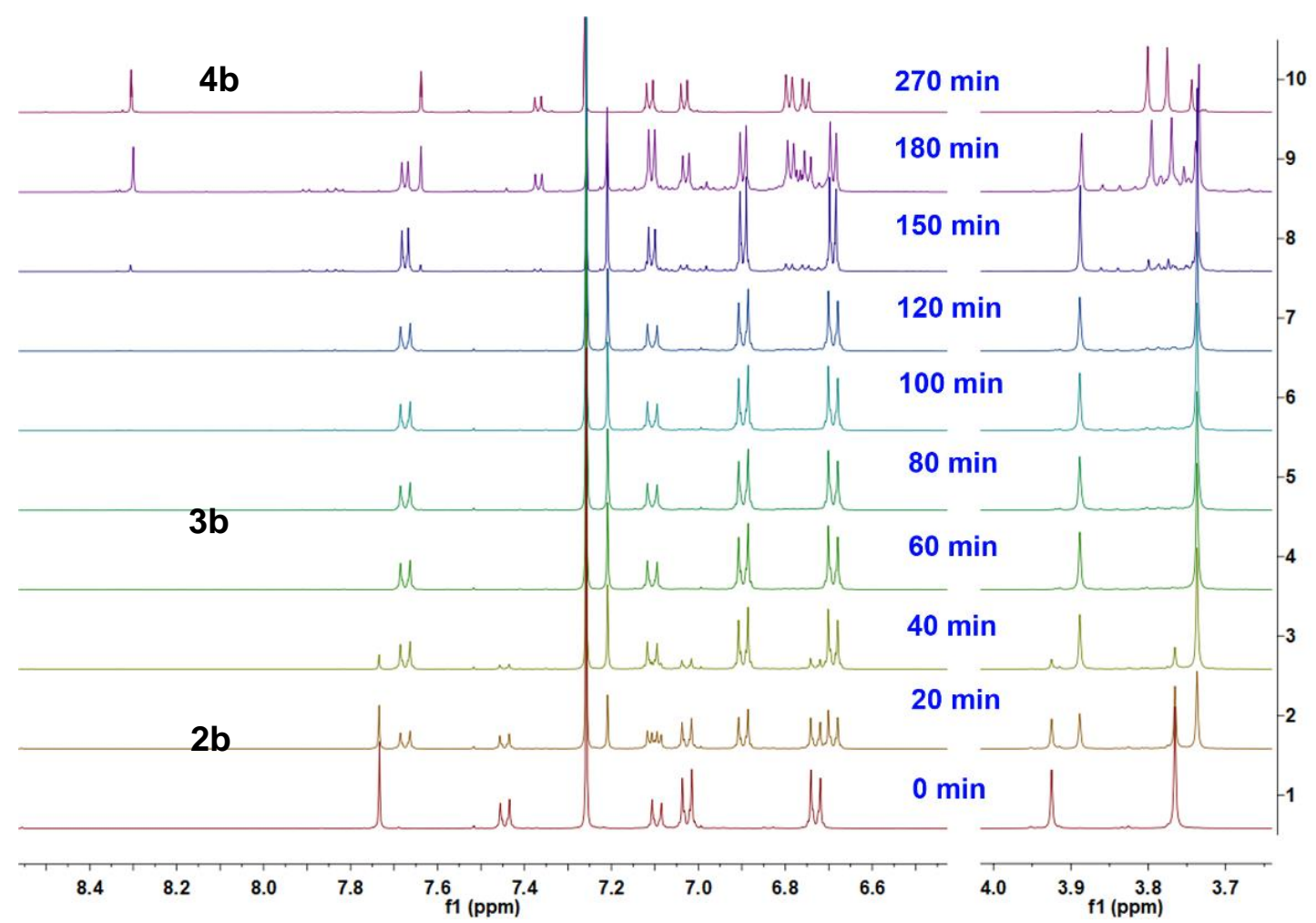

Figure $\mathbf{S 2 2}{ }^{1} \mathrm{H}$ NMR spectrum $\left(400 \mathrm{MHz}, 293 \mathrm{~K},{ }^{*} \mathrm{CDCl}_{3}\right)$ of compound $\mathbf{2 b}$ to $\mathbf{4 b}$ under UV irradiation $\left(\lambda_{\mathrm{ex}}=365 \mathrm{~nm}, 6 \mathrm{~W}\right)$ with different time. 


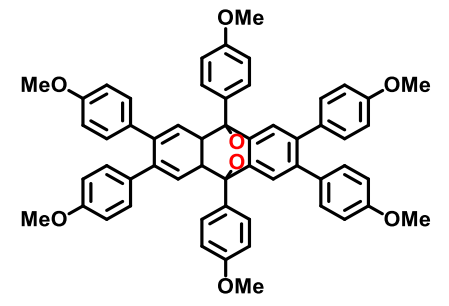

$\mathbf{3 b}$

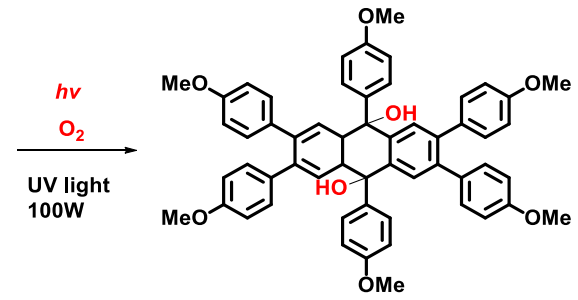

4b'

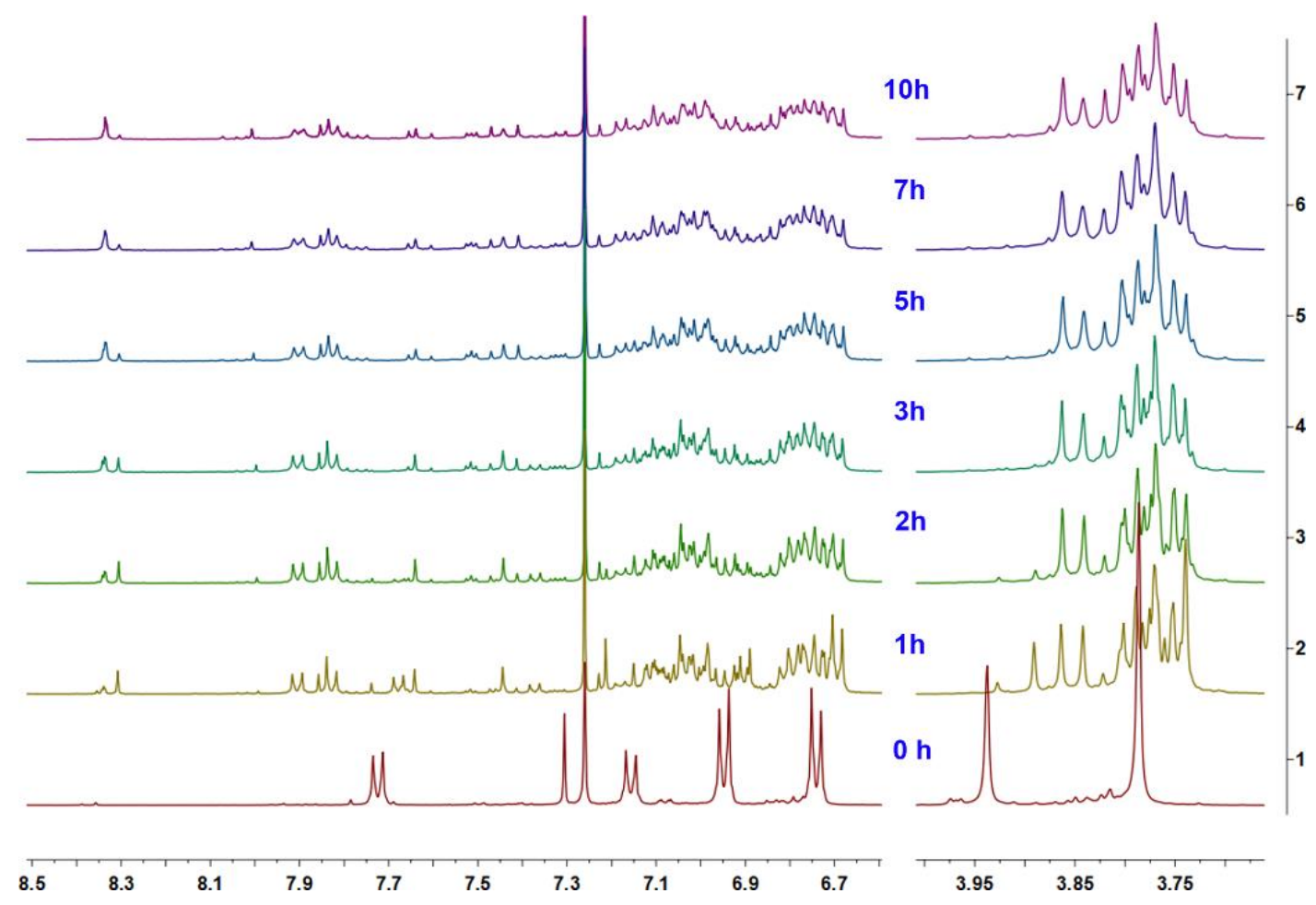

Figure $\mathbf{S 2 3}{ }^{1} \mathrm{H}$ NMR spectrum $\left(400 \mathrm{MHz}, 293 \mathrm{~K},{ }^{*} \mathrm{CDCl}_{3}\right)$ of compound $\mathbf{3 b}$ to $\mathbf{4 b}$ ' under UV irradiation $\left(\lambda_{\mathrm{ex}}=365 \mathrm{~nm}, 6 \mathrm{~W}\right)$ with different time in the presence of $\mathrm{O}_{2}$.

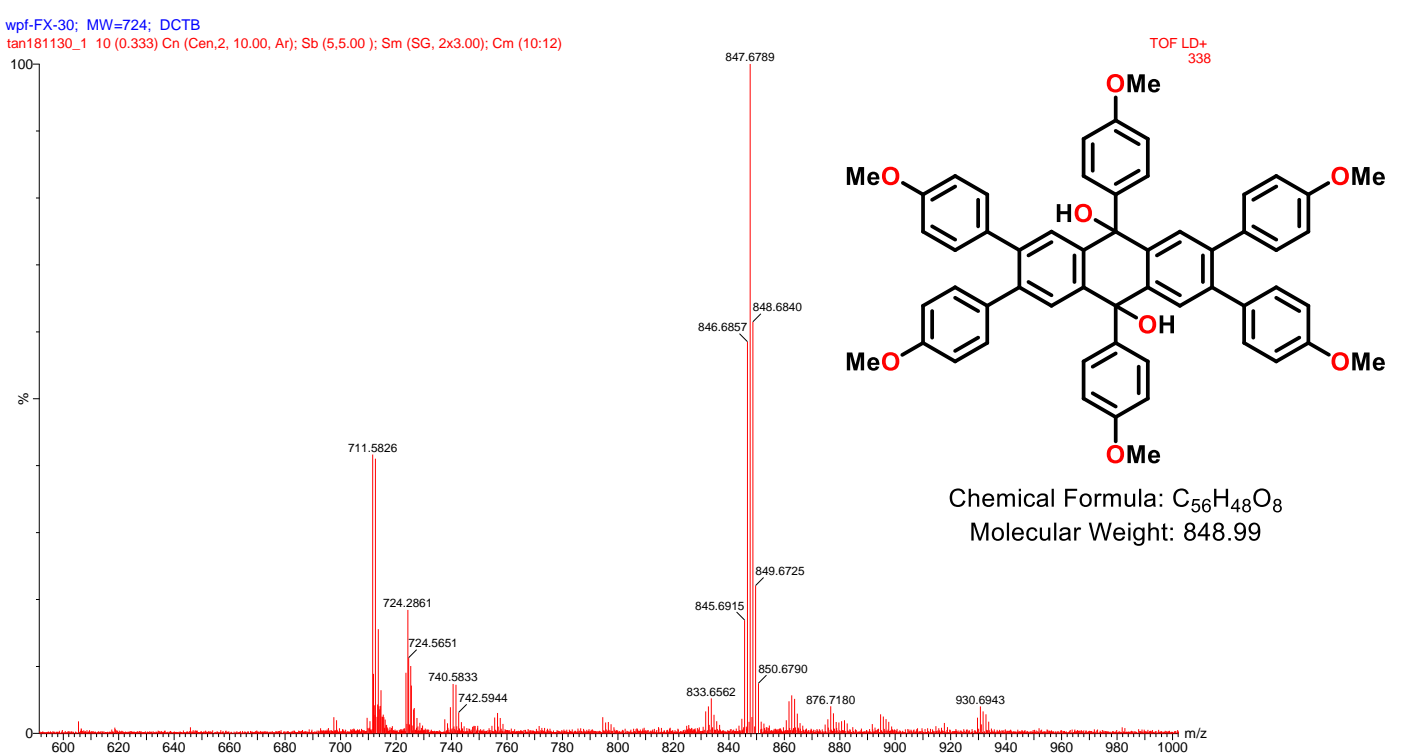

Figure S24 the HRMS spectra of endoperoxides $4 \mathbf{b}$ ' under UV-irradiation in the presence of $\mathrm{O}_{2}$. 


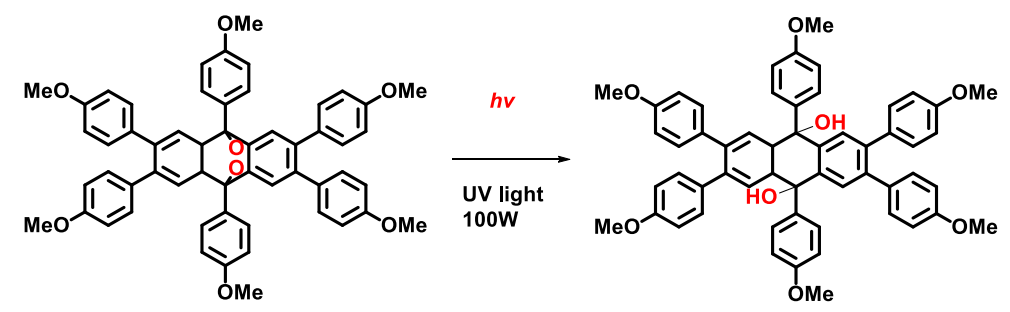

3b

$4 \mathbf{b}^{\prime}$

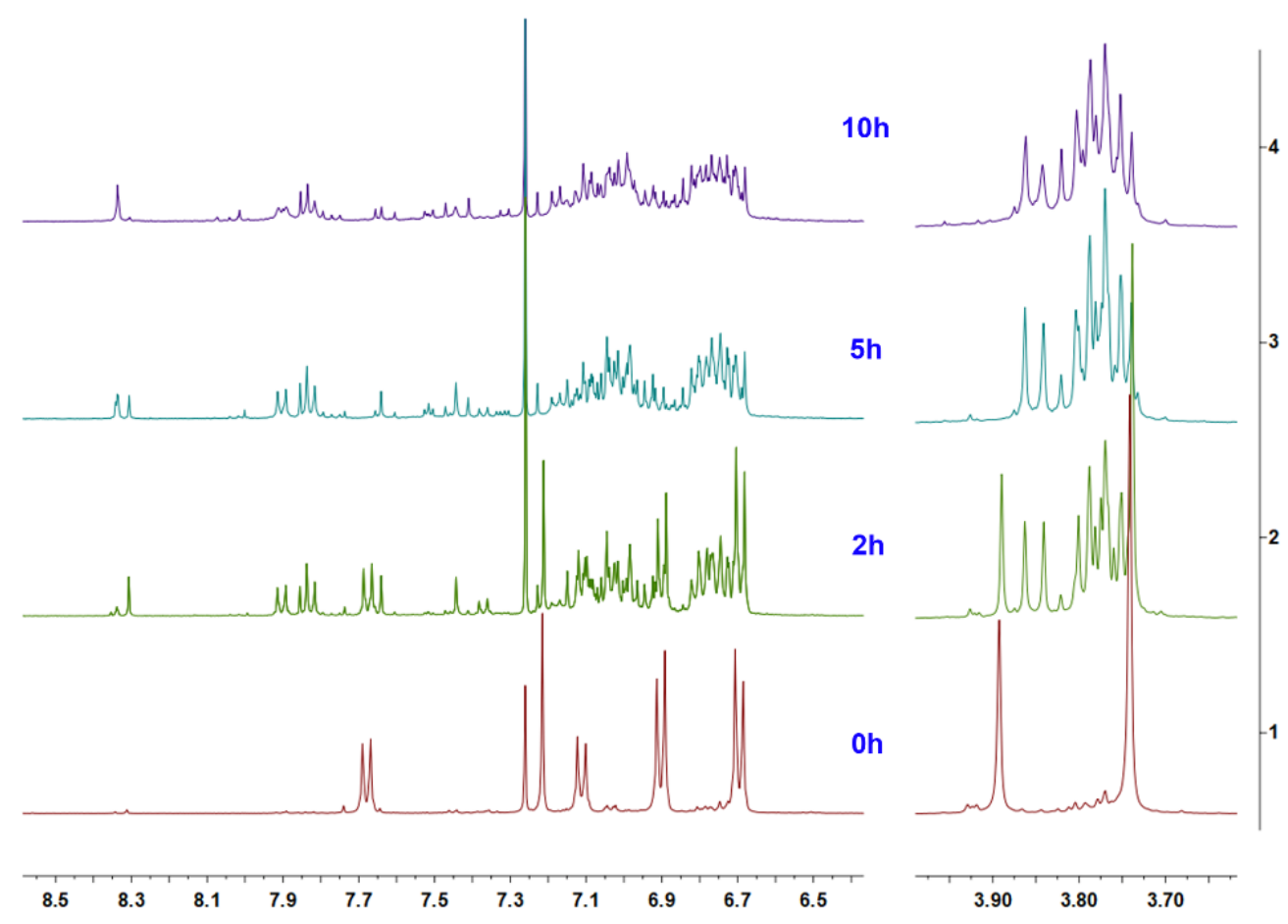

Figure S25 ${ }^{1} \mathrm{H}$ NMR spectrum $\left(400 \mathrm{MHz}, 293 \mathrm{~K},{ }^{*} \mathrm{CDCl}_{3}\right)$ of compound $\mathbf{3 b}$ to $\mathbf{4 b}$ ' under UV irradiation $\left(\lambda_{\mathrm{ex}}=365 \mathrm{~nm}, 6 \mathrm{~W}\right)$ with different time in the absence of $\mathrm{O}_{2}$.

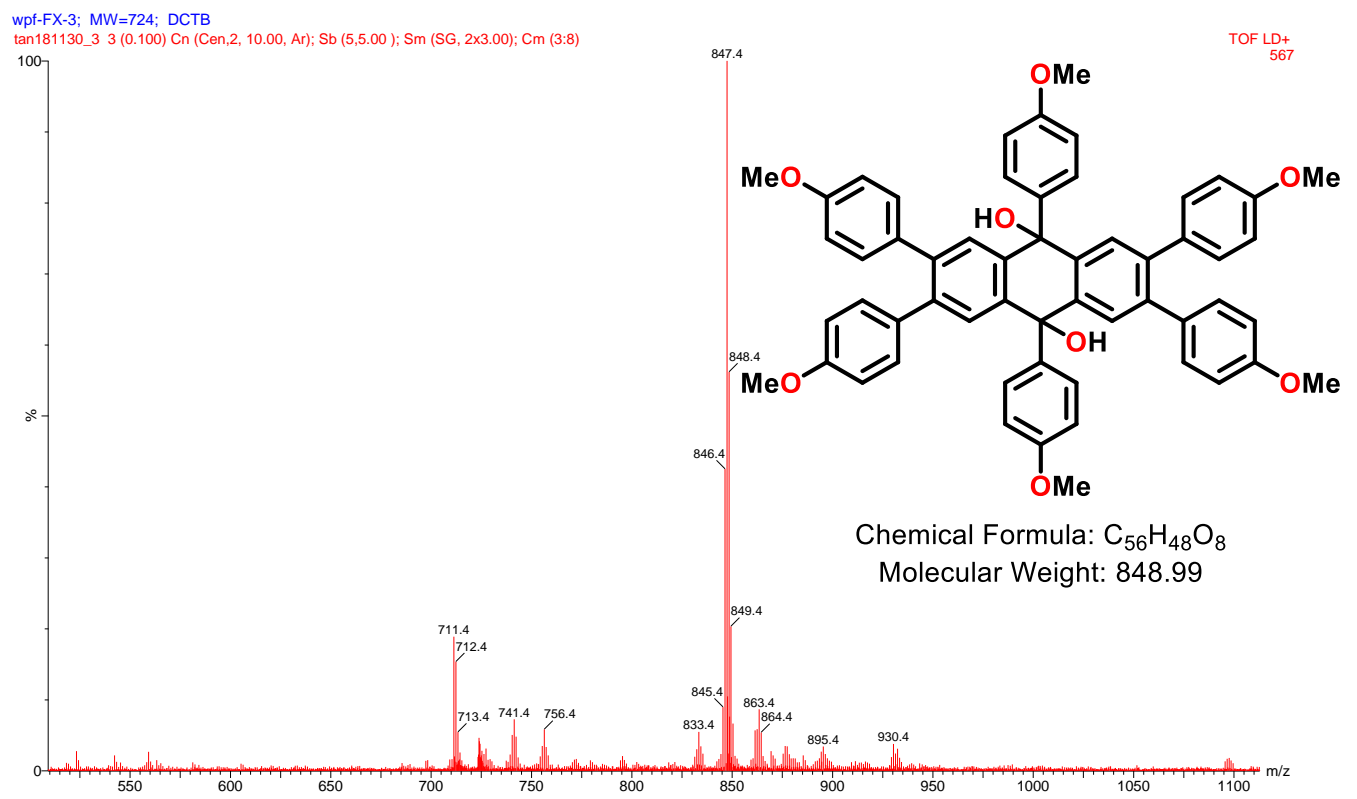

Figure S26 the HRMS spectra of endoperoxides $\mathbf{3 b}$ under UV-irradiation in the absence of $\mathrm{O}_{2}$. 


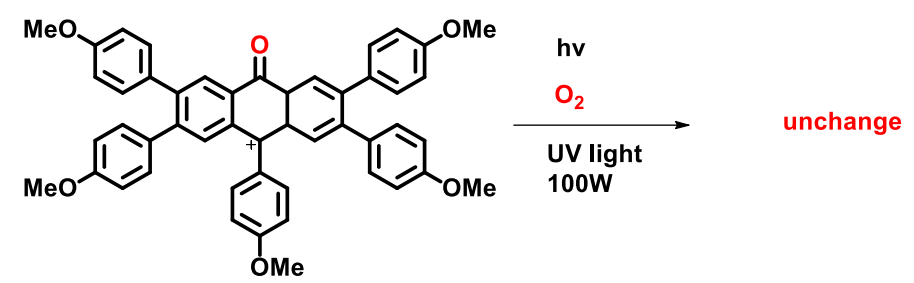

$5 \mathbf{b}$

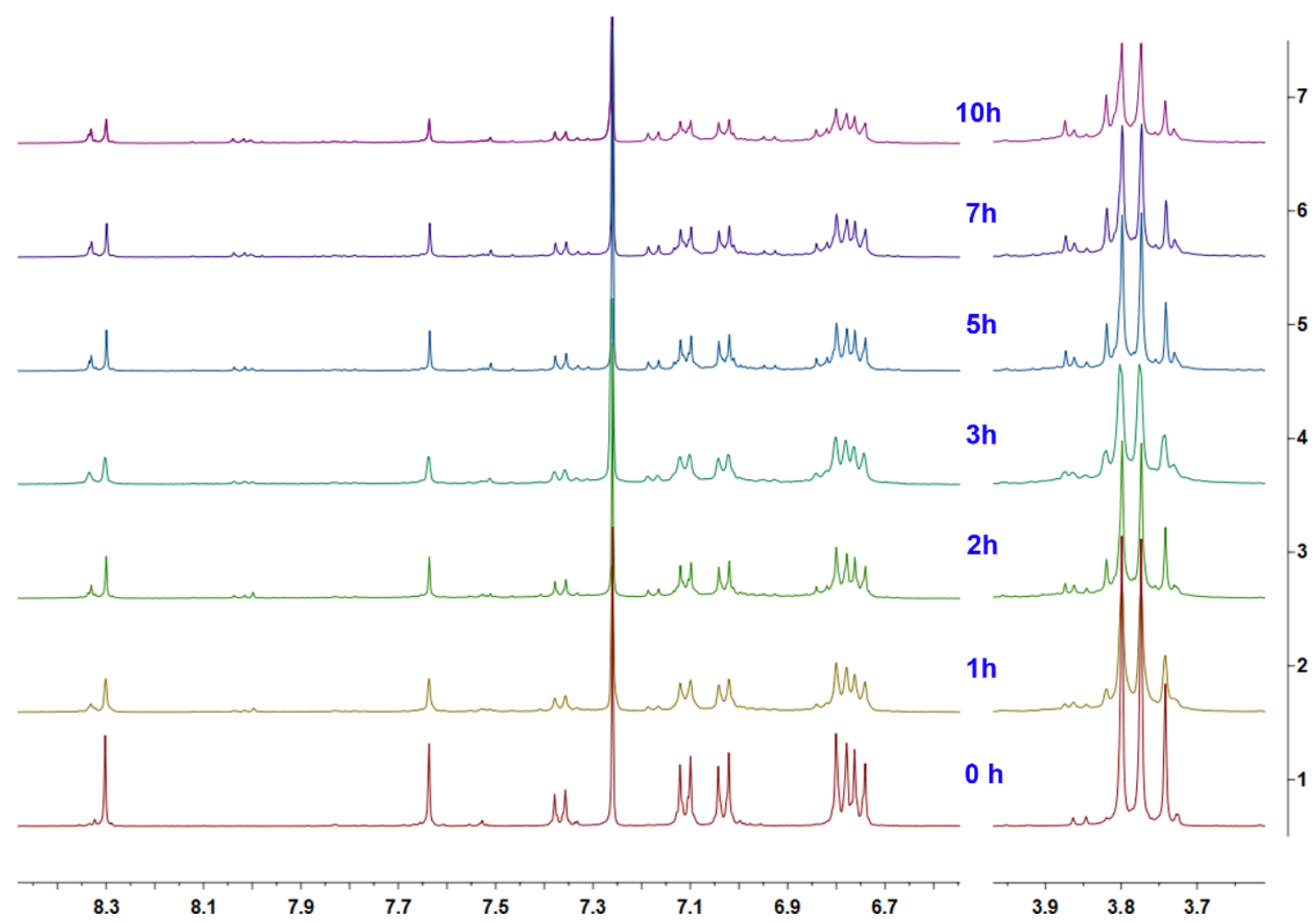

Figure S27 ${ }^{1} \mathrm{H}$ NMR spectrum $\left(400 \mathrm{MHz}, 293 \mathrm{~K},{ }^{*} \mathrm{CDCl}_{3}\right)$ of compound $\mathbf{5 b}$ under UV irradiation $\left(\lambda_{\mathrm{ex}}=365 \mathrm{~nm}, 100 \mathrm{~W}\right)$ with different time.

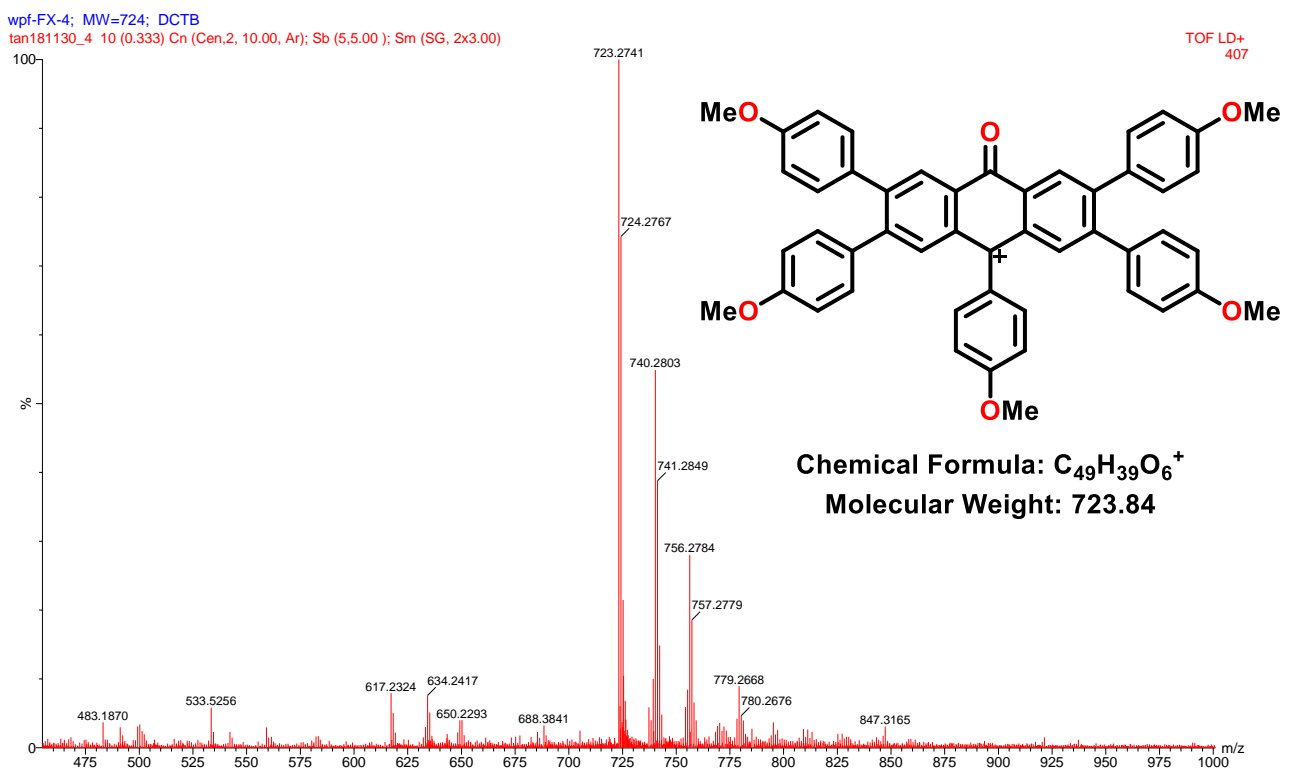

Figure S28 the HRMS spectra of semiquinone radical 5b under UV-irradiation in the presence of $\mathrm{O}_{2}$ 


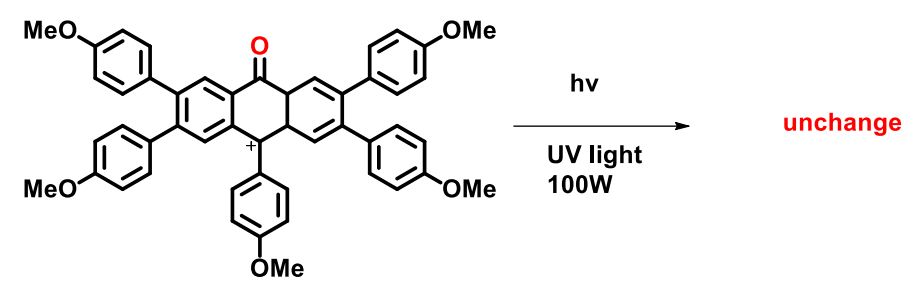

4b

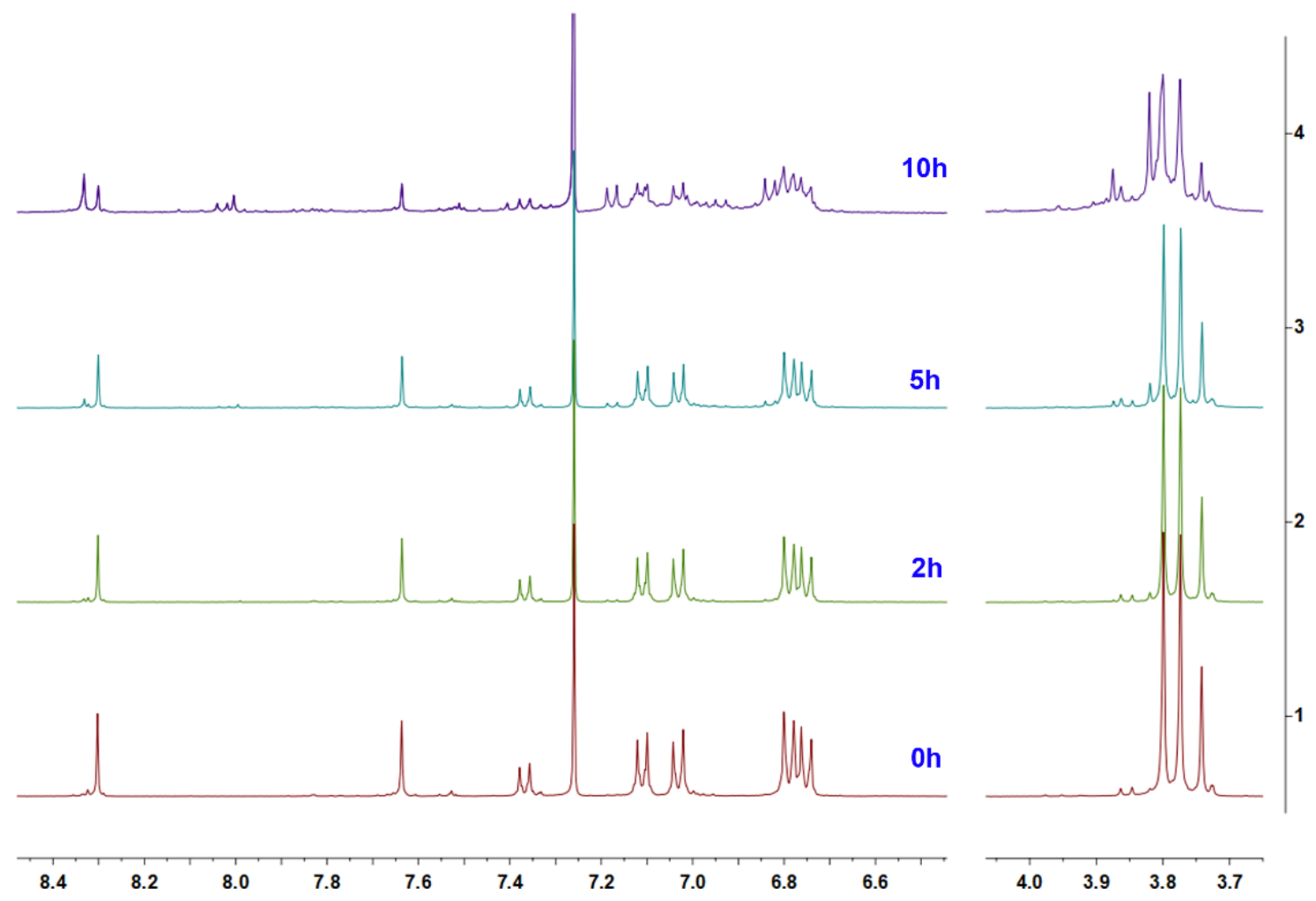

Figure S29 the HRMS spectra of semiquinone radical $\mathbf{5 b}$ under UV-irradiation in the absence of $\mathrm{O}_{2}$

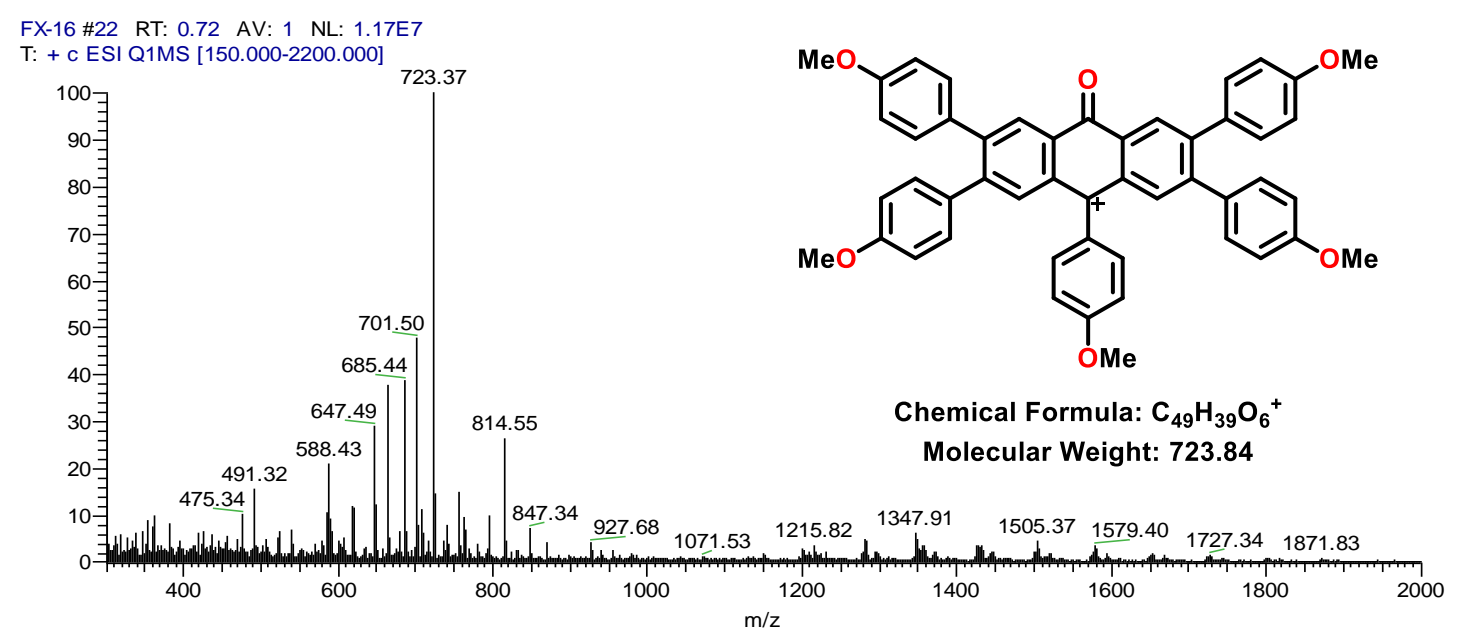

Figure S30 the HRMS spectra of semiquinone radical $\mathbf{5 b}$ under UV-irradiation in the absence of $\mathrm{O}_{2}$ 


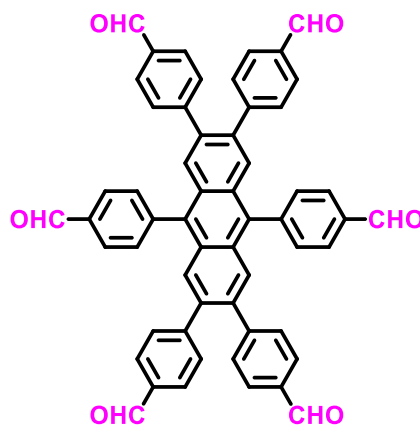

2c

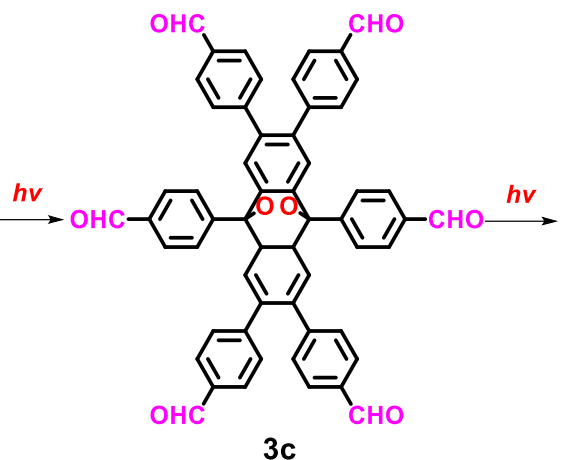

$3 c$

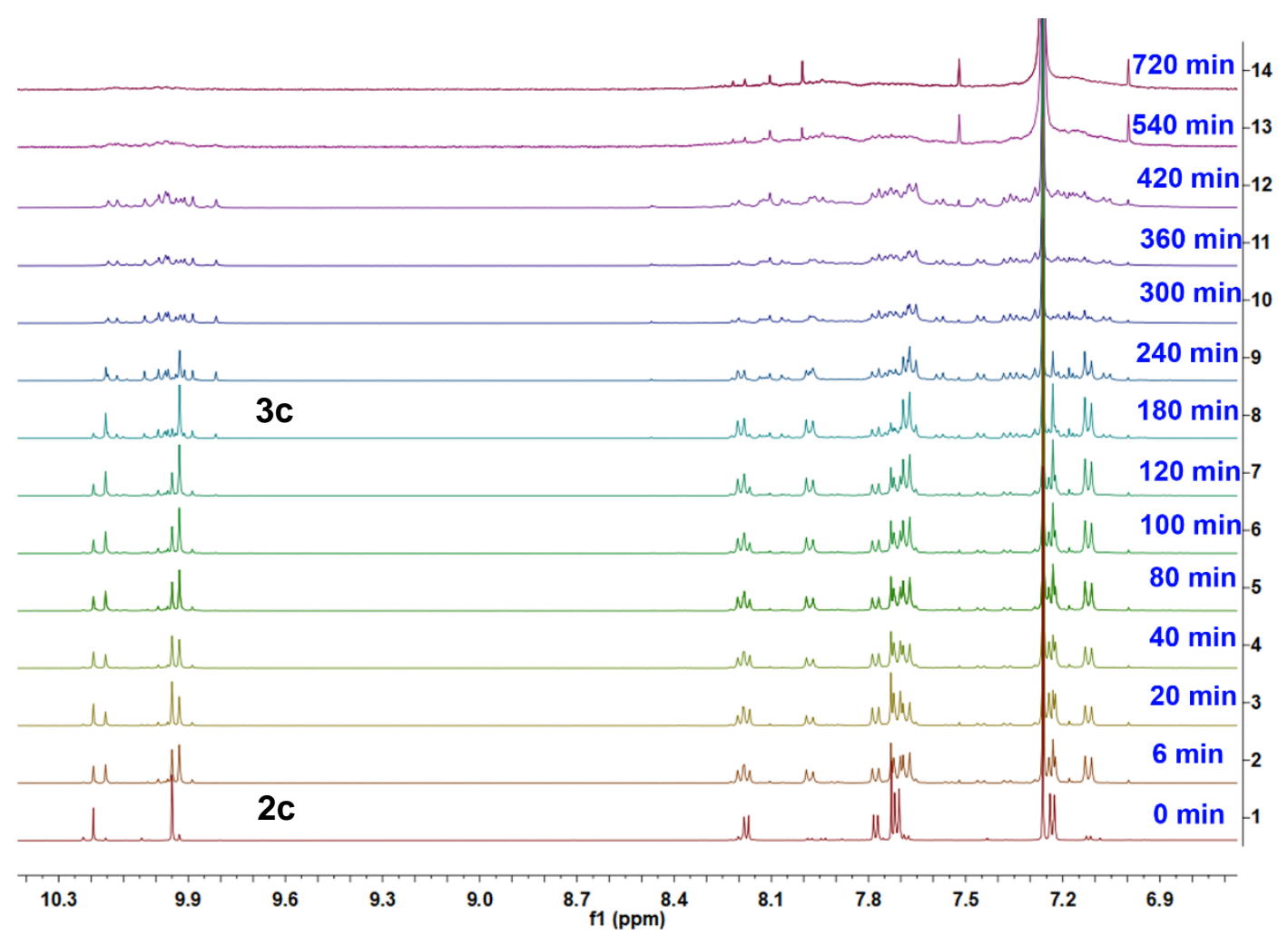

Figure S31 ${ }^{1} \mathrm{H}$ NMR spectrum $\left(400 \mathrm{MHz}, 293 \mathrm{~K},{ }^{*} \mathrm{CDCl}_{3}\right)$ of compound $\mathbf{2 c}$ under UV irradiation $\left(\lambda_{\mathrm{ex}}=365 \mathrm{~nm}\right)$ with different time. 


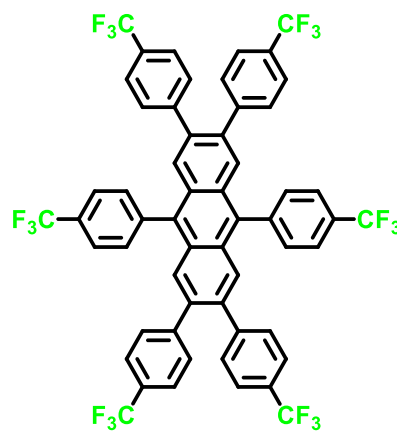

2d

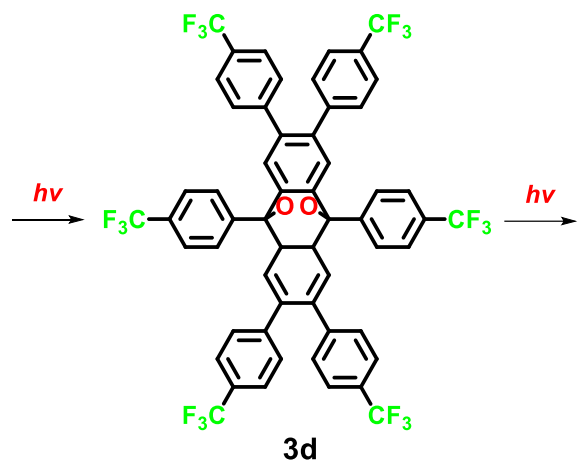

$3 d$

decomposition

\section{$3 d$}

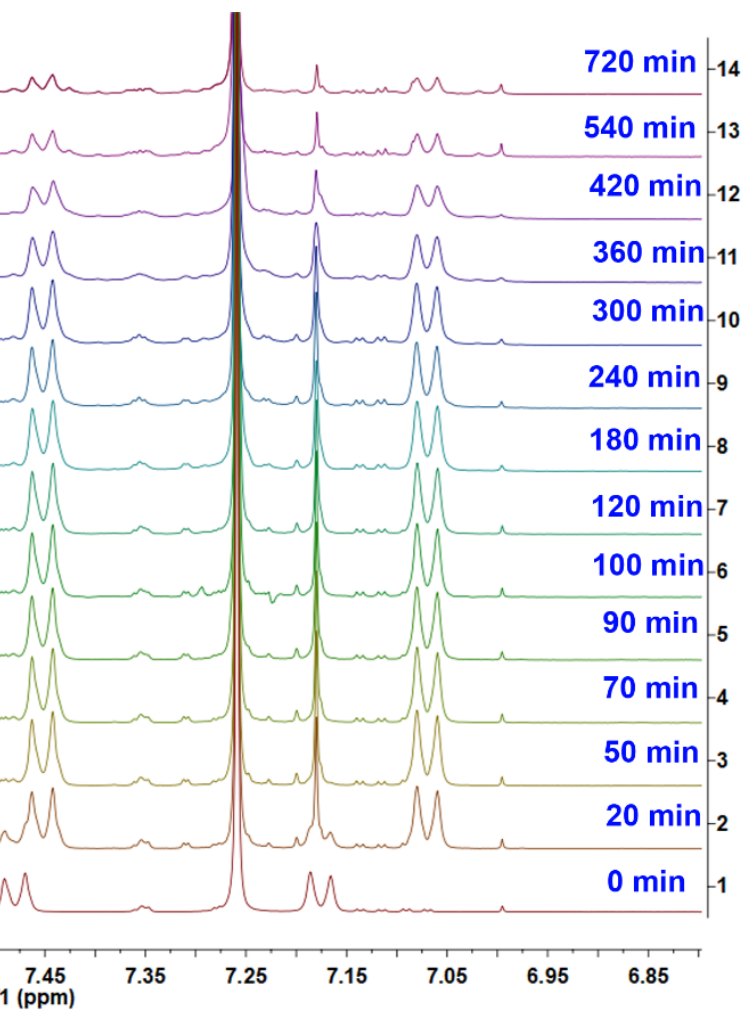

Figure S32 ${ }^{1} \mathrm{H}$ NMR spectrum $\left(400 \mathrm{MHz}, 293 \mathrm{~K},{ }^{*} \mathrm{CDCl}_{3}\right)$ of compound $\mathbf{2 d}$ under UV irradiation $\left(\lambda_{\mathrm{ex}}=365 \mathrm{~nm}\right)$ with different time 


\subsection{Variable-temperature ${ }^{1} \mathrm{H}$ NMR spectrum}

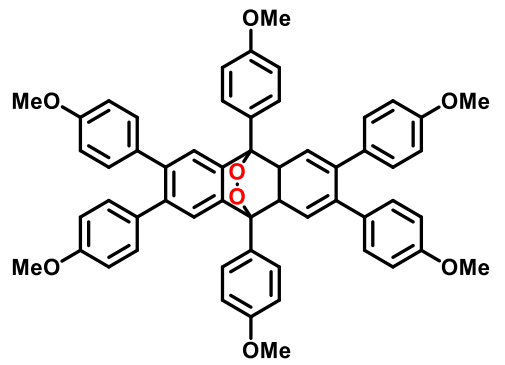

3b

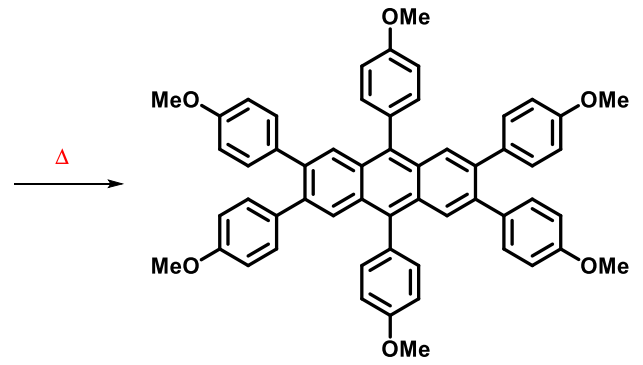

2b

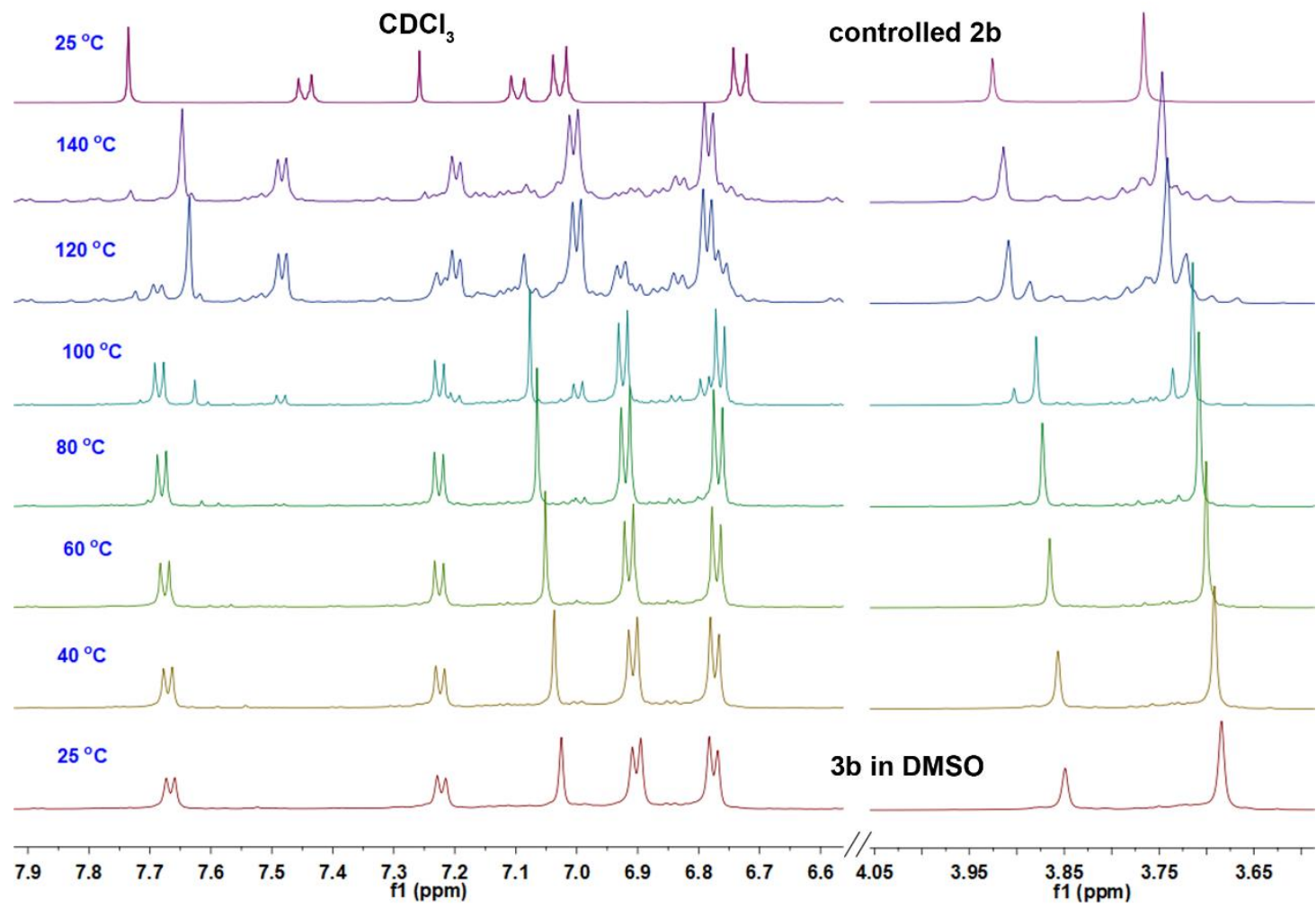

Figure S33 Variable-temperature ${ }^{1} \mathrm{H}$ NMR $\left(100 \mathrm{MHz},{ }^{*} \mathrm{DMSO}\right)$ for endoperoxides $\mathbf{3 b}$ from $25^{\circ} \mathrm{C}$ to $140{ }^{\circ} \mathrm{C}$. 


\section{FT-IR Spectrum}

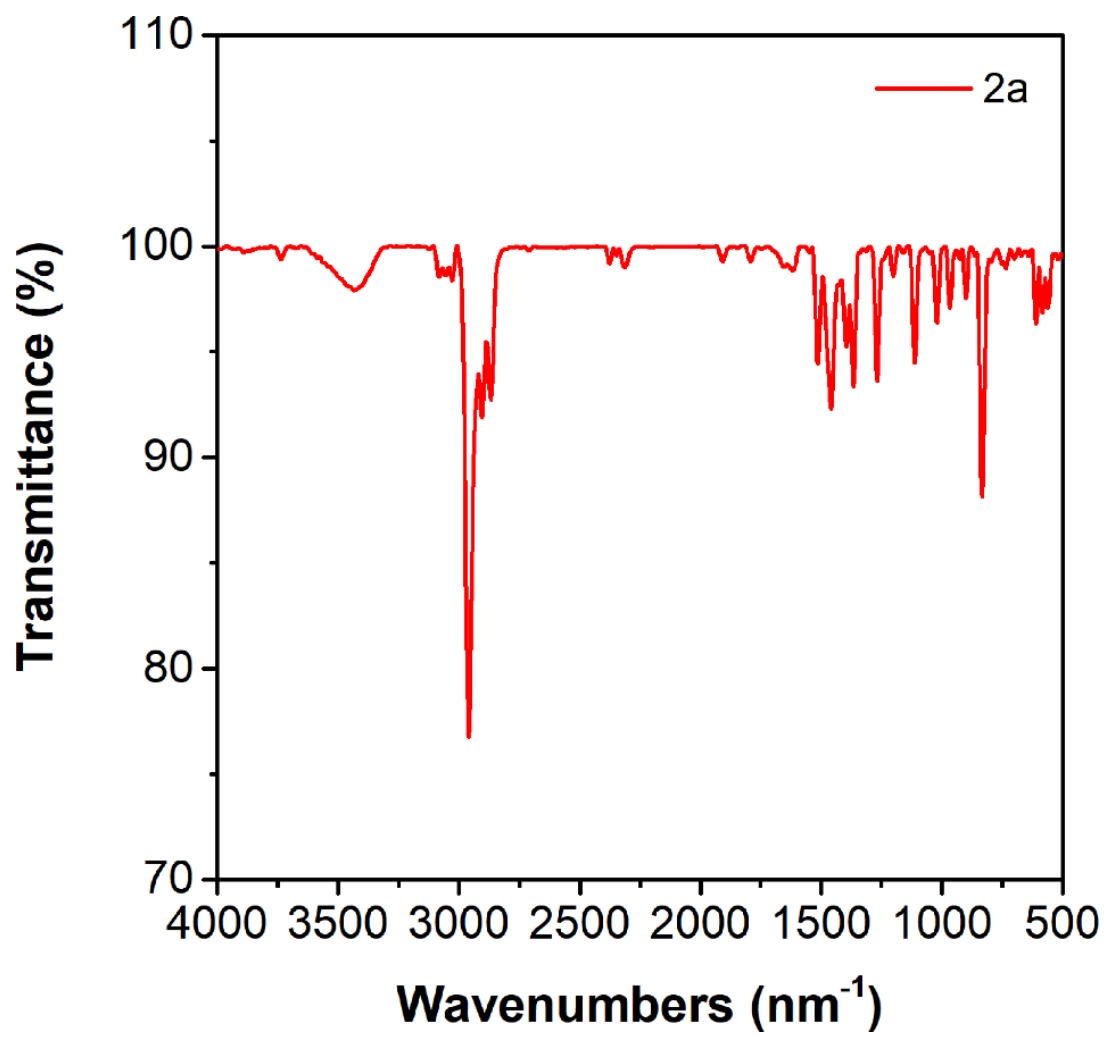

Figure S34 FT-IR spectra of compound 2a

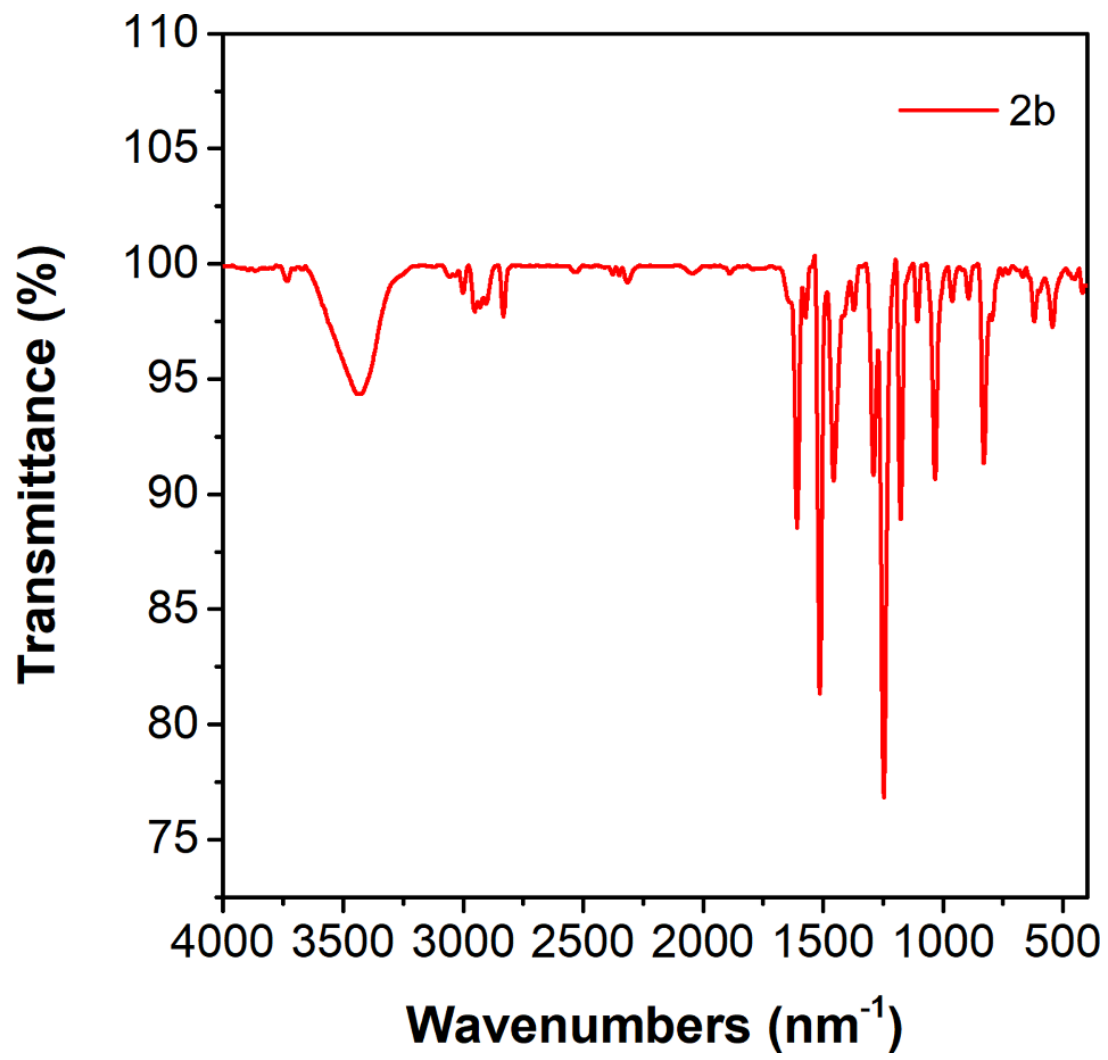

Figure S35 FT-IR spectra of compound 2b 


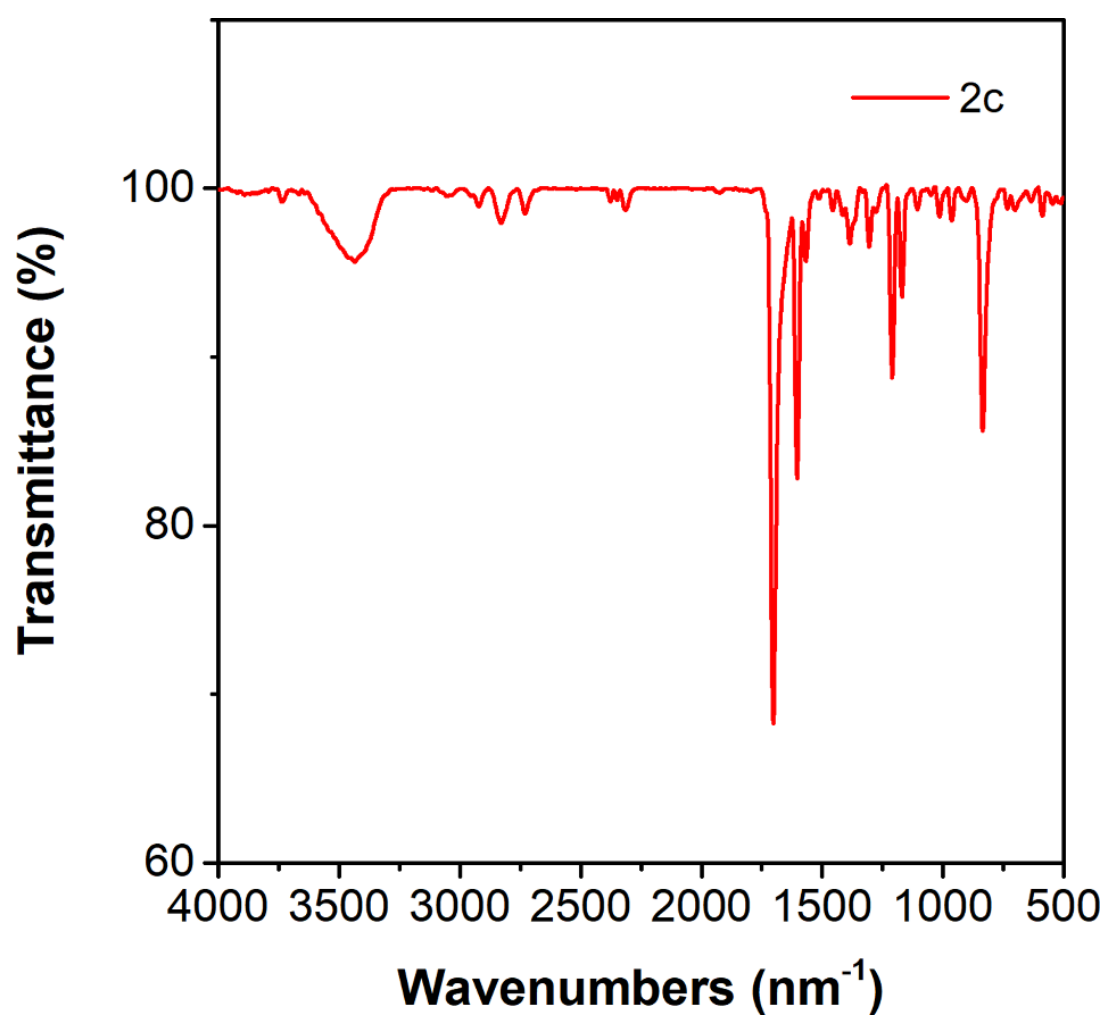

Figure S36 FT-IR spectra of compound 2c

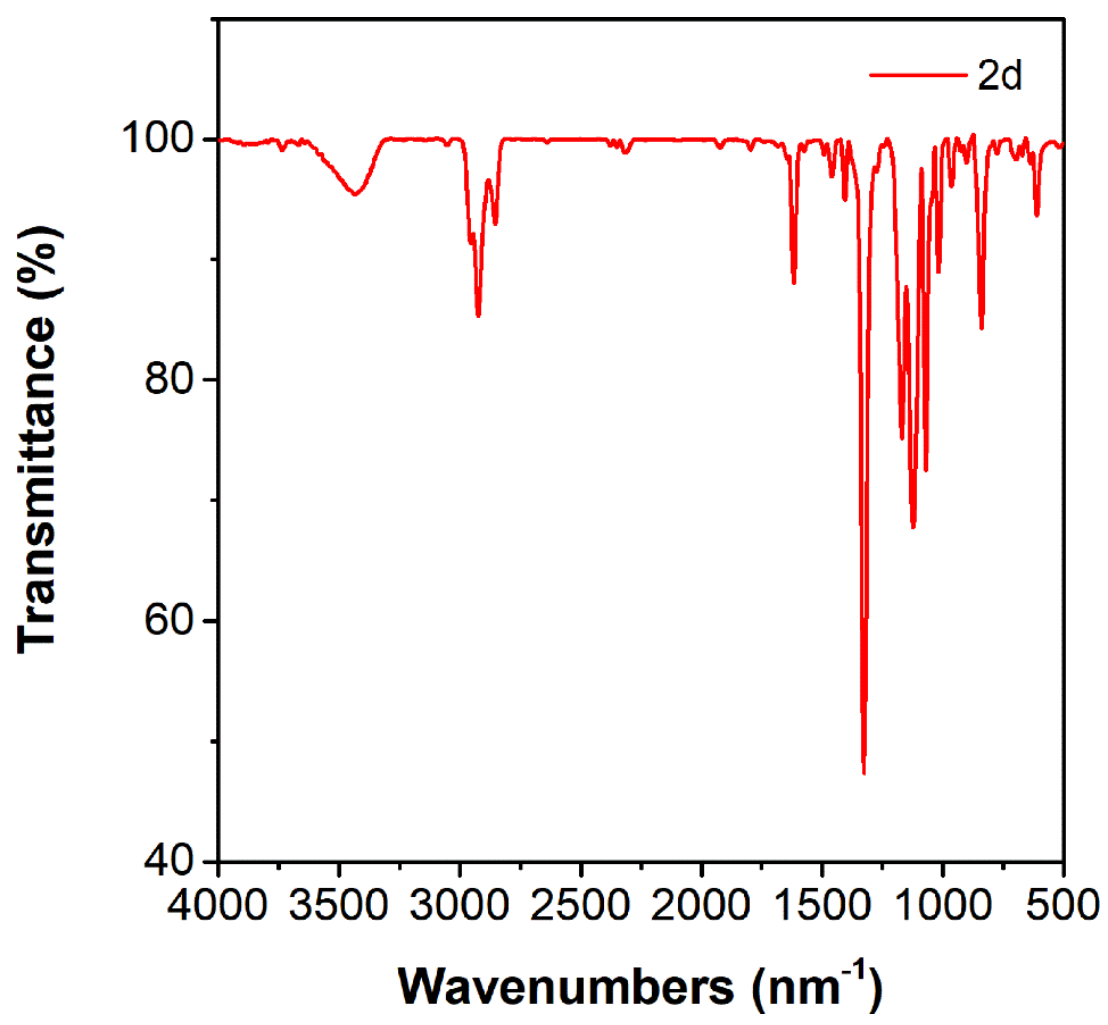

Figure S37 FT-IR spectra of compound 2d 


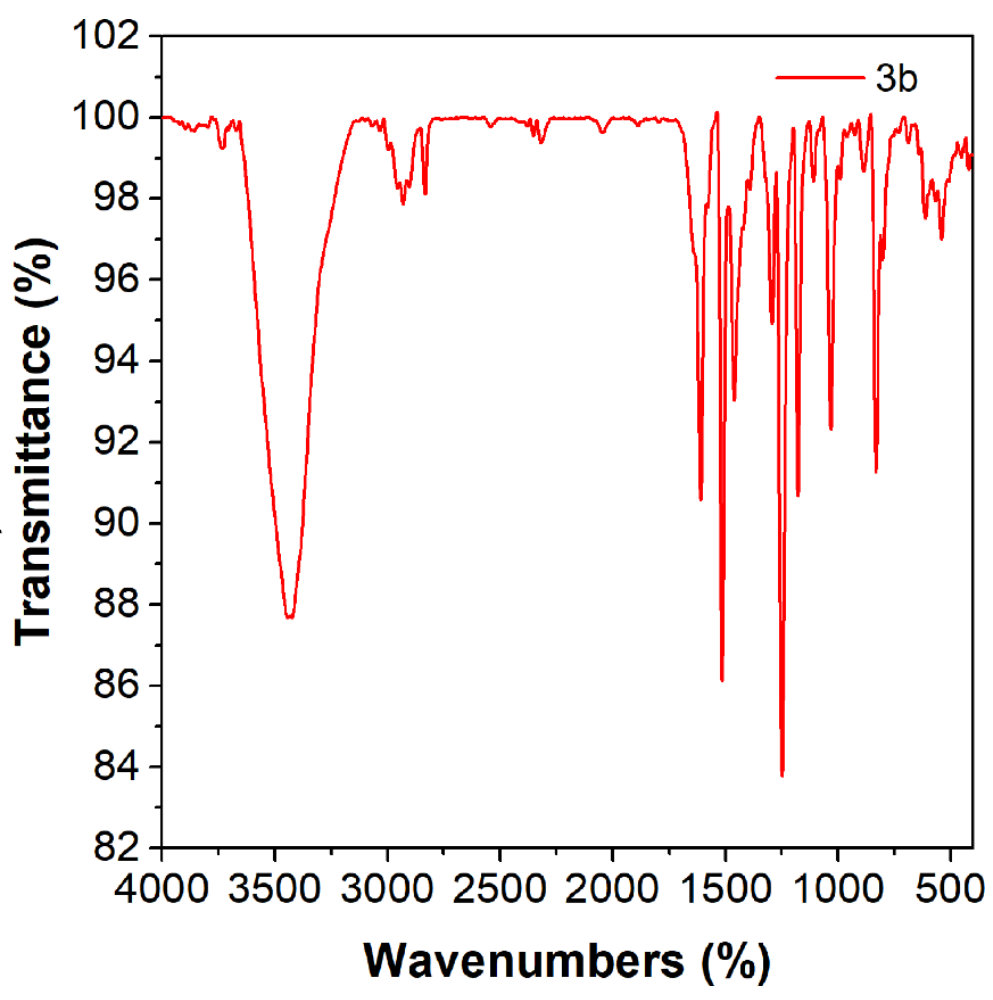

Figure S38 FT-IR spectra of compound $\mathbf{3 b}$.

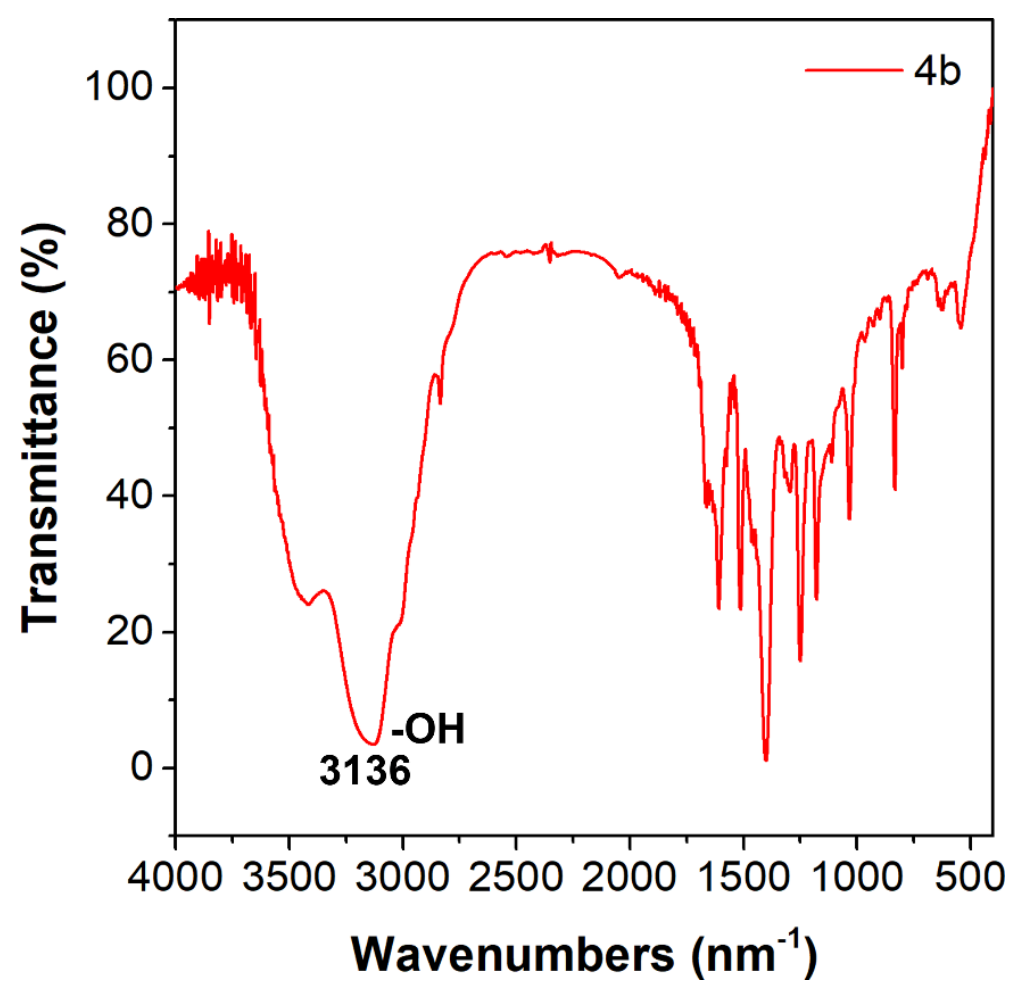

Figure S39 FT-IR spectra of compound $\mathbf{4 b}$. 


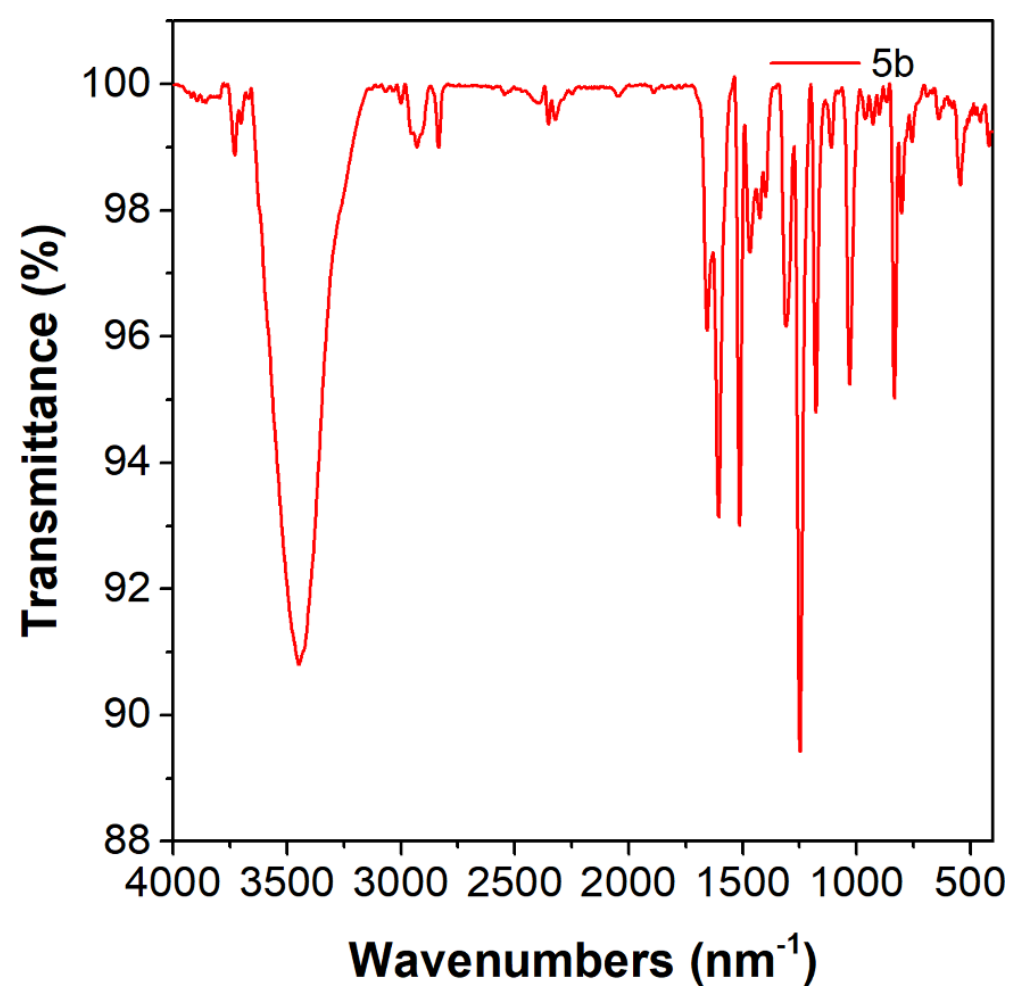

Figure S40 FT-IR spectra of compound $\mathbf{5 b}$.

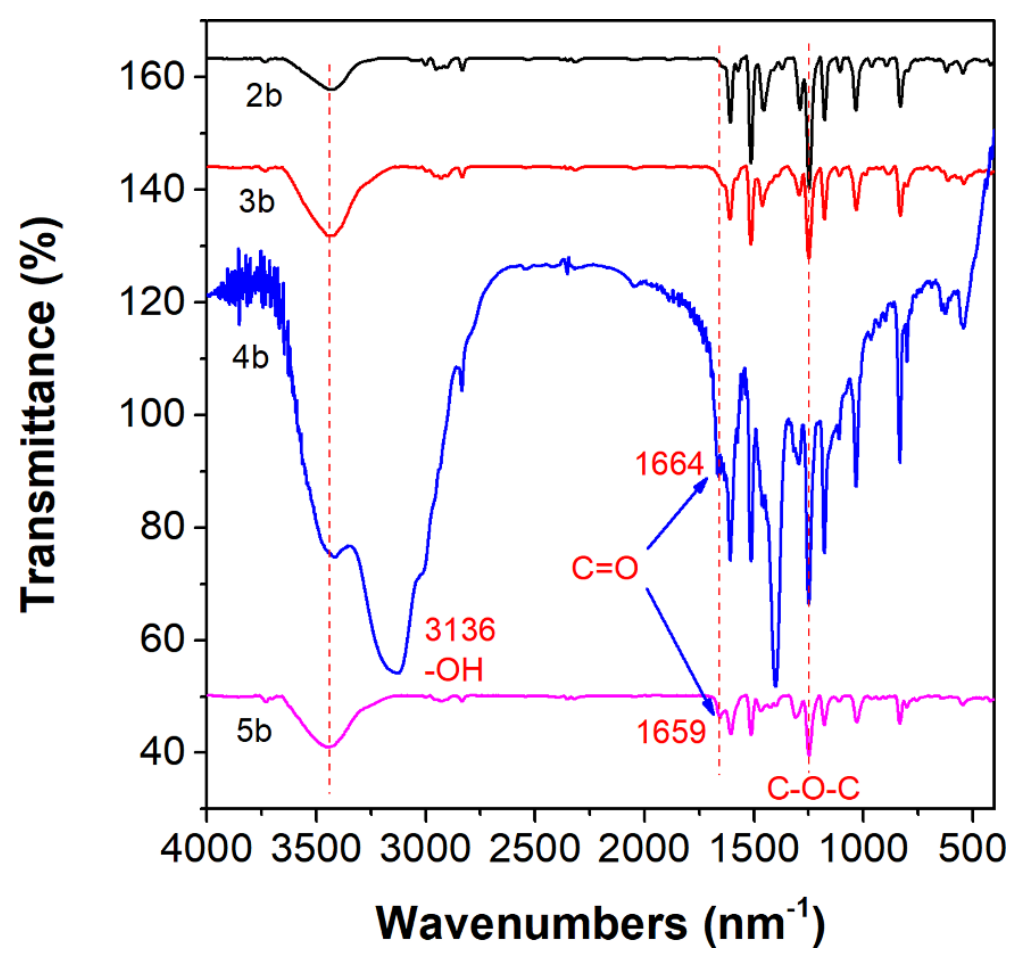

Figure S41 FT-IR spectra of compounds $\mathbf{2 b}, \mathbf{3 b}, \mathbf{4 b}$ and $\mathbf{5 b}$. 


\section{EPR spectra}

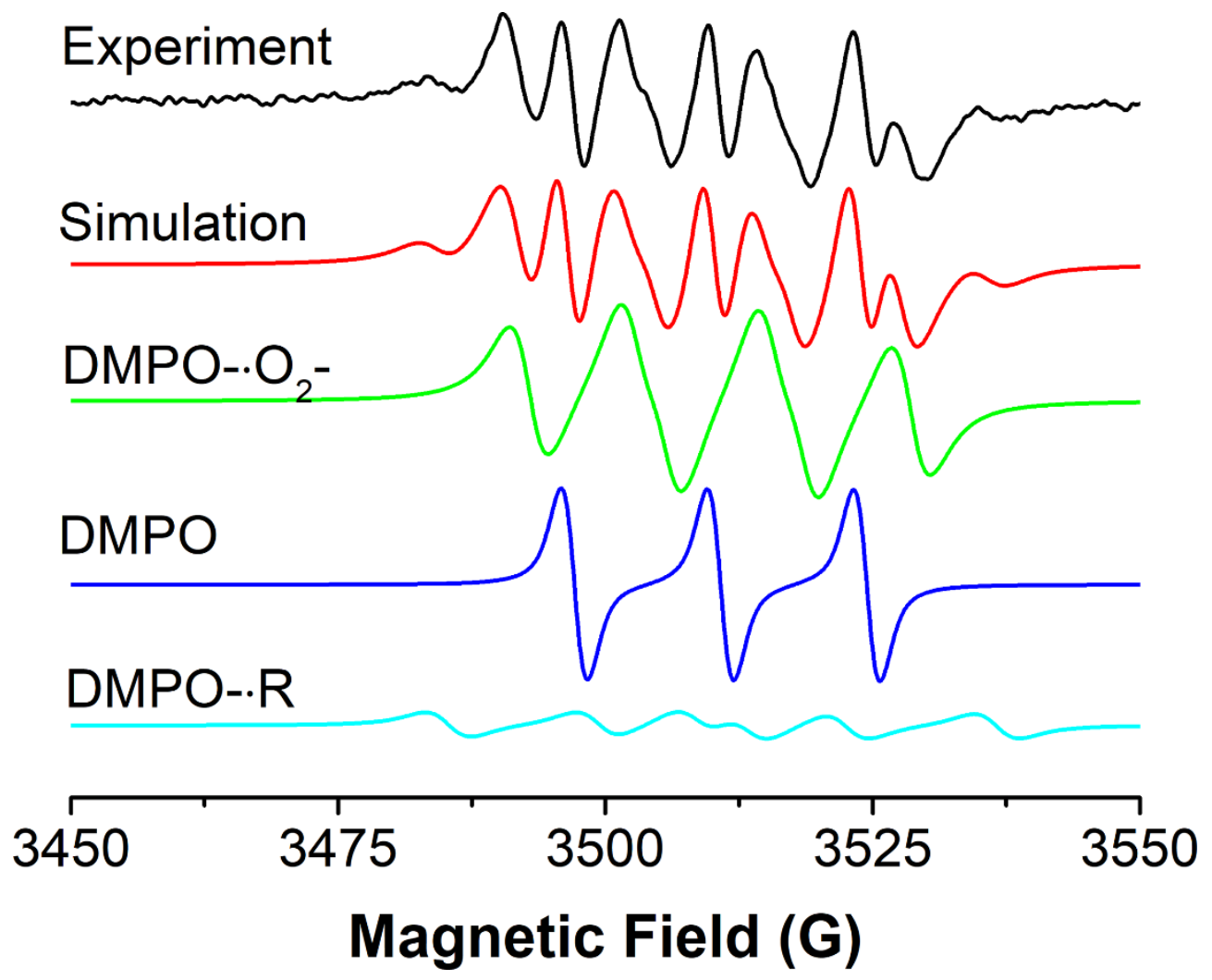

Figure S42 EPR signals to evaluate the production of superoxide radical (O2-), selfphotolysis of DMPO radical, and an alkyl radical for $\mathbf{3 b}$ in toluene under UV irradiation. 


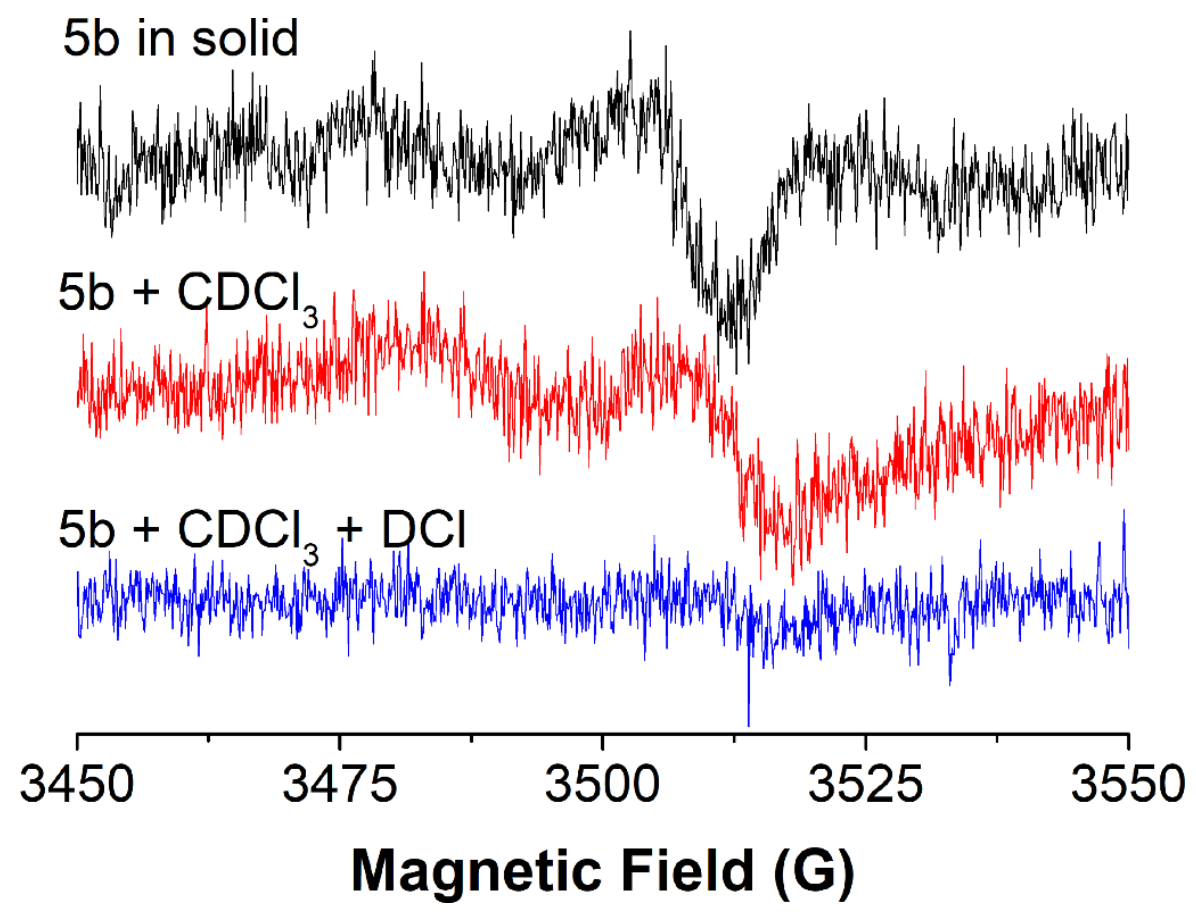

Figure $\mathrm{S43}$ EPR spectra of $\mathbf{5 b}$ in the solid state, $\mathrm{CDCl}_{3}$ and mixture solution of $\mathrm{CDCl}_{3}$ and $\mathrm{DCl}\left(\mathrm{DCl}, 35 \%\right.$ in $\left.\mathrm{D}_{2} \mathrm{O}\right)$. 


\section{Mass Spectrum Analysis}

fx-72, MW=655; $\mathrm{CH}_{4}$

$\tan 170214 \_984(1.400) \mathrm{Cm}(83: 86-(1: 80+88: 118))$

100

662.7216

TOF MS Cl+

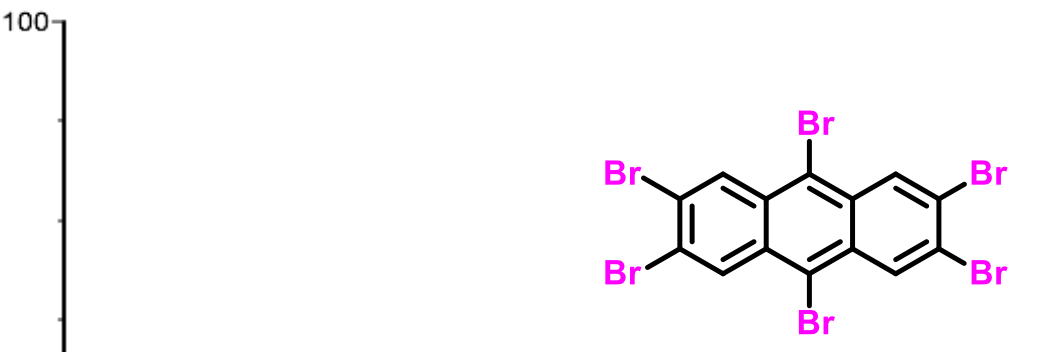

Chemical Formula: $\mathrm{C}_{14} \mathrm{H}_{4} \mathrm{Br}_{6}$

Molecular Weight: 651.61

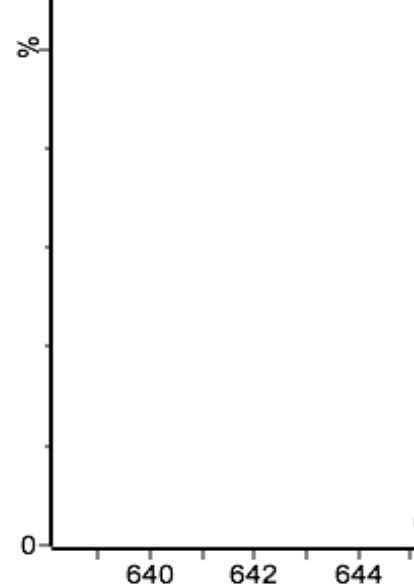

647.6909

655.8383

$653.8282 \quad 657.837$

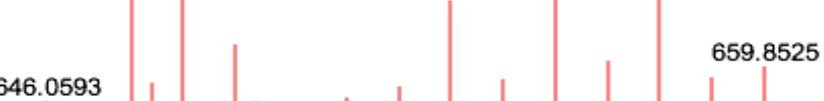

$\begin{array}{llllllllllll}640 & 642 & 644 & 646 & 648 & 650 & 652 & 654 & 656 & 658 & 660 & 662\end{array}$

Figure S44 the HRMS spectra of 2,3,6,7,9,10-hexa-bromo anthracene 1 
FX95 \#9 RT: $0.08 \quad$ AV: $1 \quad$ NL: $2.21 \mathrm{E} 7$

T: FTMS + p ESI Full ms [150.0000-1500.0000]

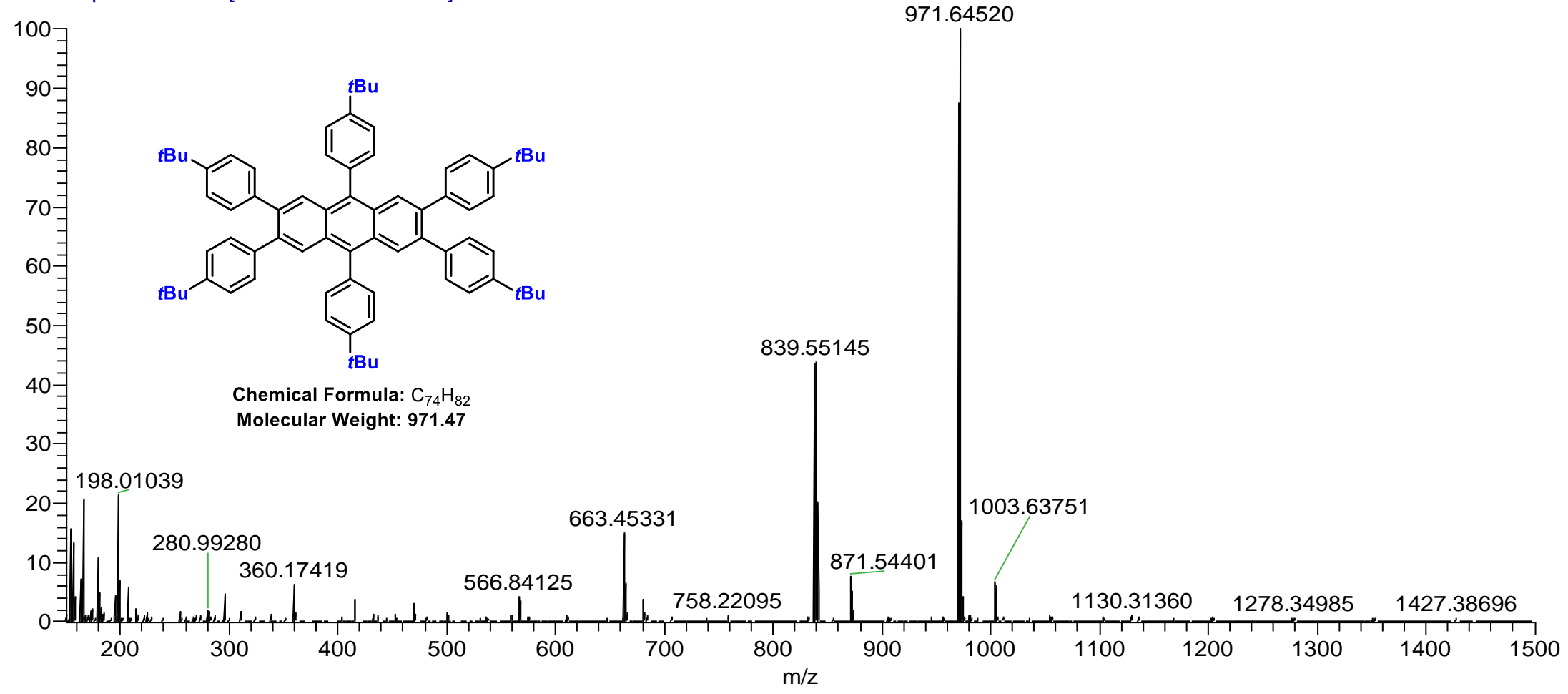

Figure $\mathbf{S 4 5}$ the HRMS spectra of 2,3,6,7,9,10-hexa-(4-tert-butyl-phenyl) anthracene 2a 
$\begin{array}{llllll}\text { FX115 \#13 RT: } 0.12 \text { AV: } 1 & \text { SB: } 1 & 0.04 & \text { NL: } 2.73 E 7\end{array}$ T: FTMS + p ESI Full ms [150.0000-1500.0

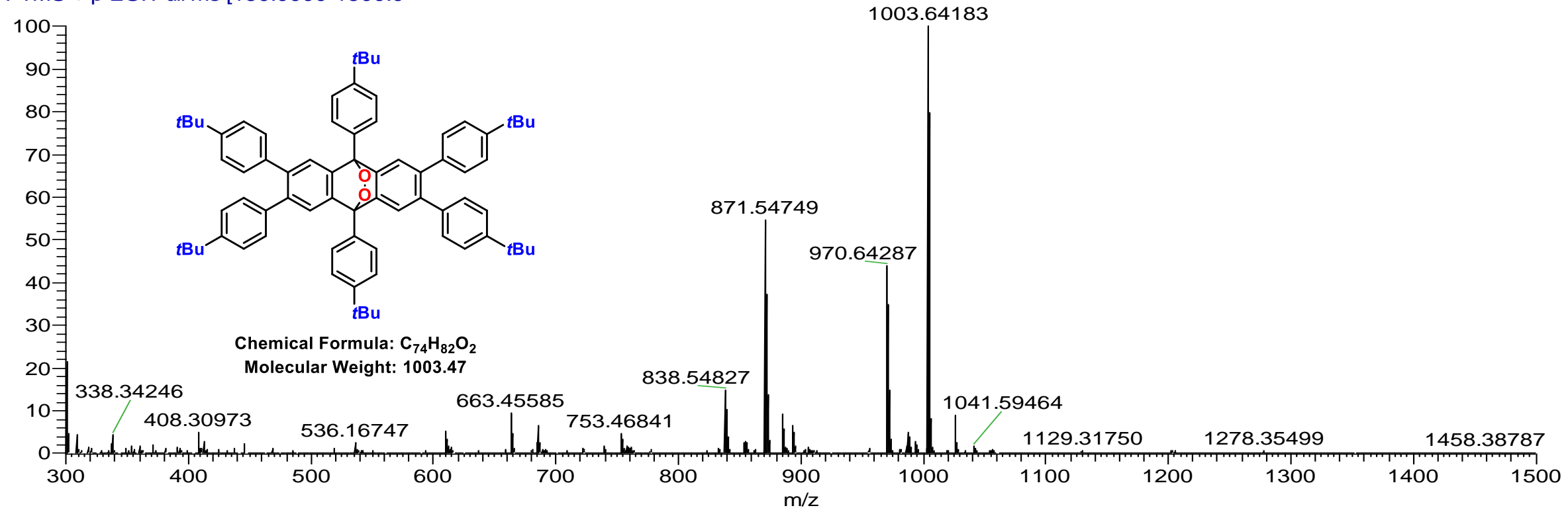

Figure S46 the HRMS spectra of endoperoxides 3a. 
FX-tBu_20191226144529 \#9 RT: 0.09 AV: $1 \quad$ NL: 2.38E7 T: FTMS + p APCl corona Full ms [200.0000-1500.

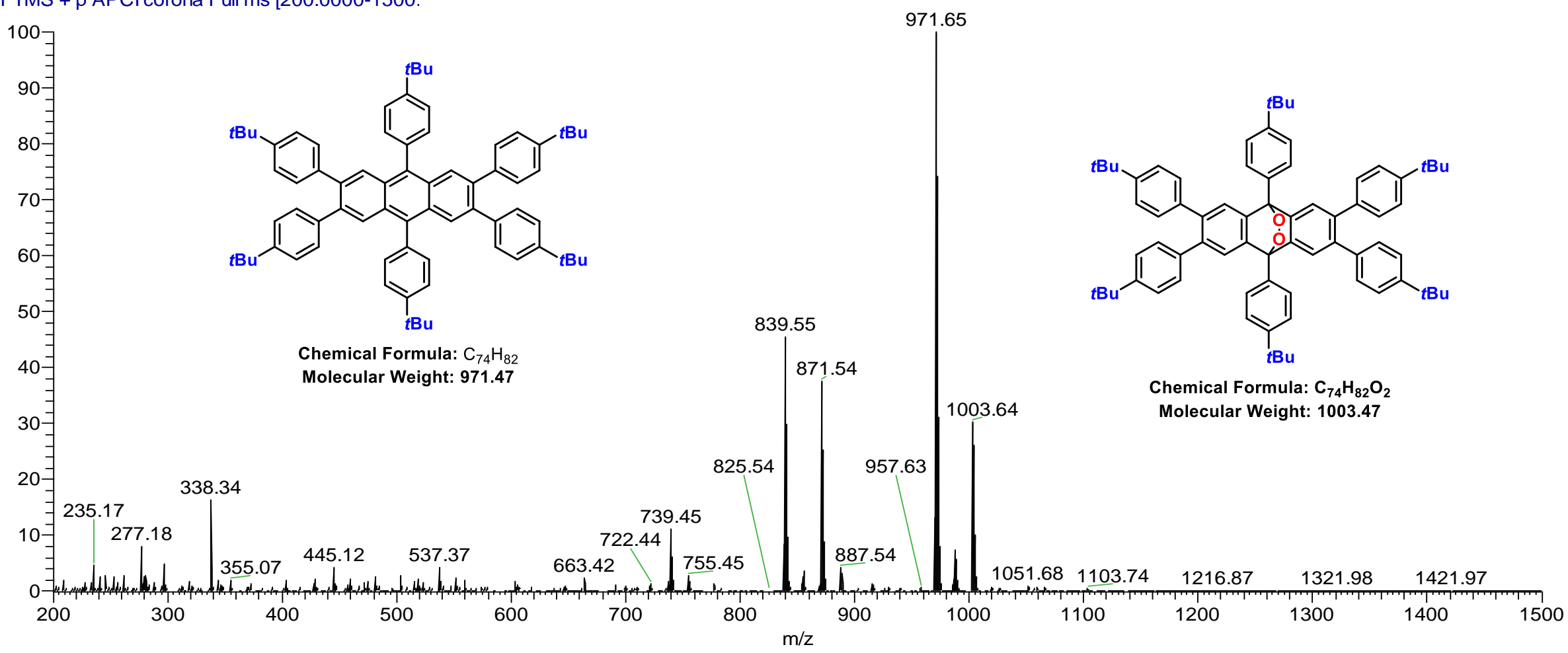

Figure S47 the HRMS spectra of 2,3,6,7,9,10-hexa-(4-tert-butyl-phenyl) anthracene 2a under UV irradiation 360 min. 
tBu-2 \#7 RT: $0.07 \quad$ AV: $1 \quad \mathrm{NL}: 1.72 \mathrm{E} 7$

T: FTMS + p APCI corona Full ms [200.0000-1500.

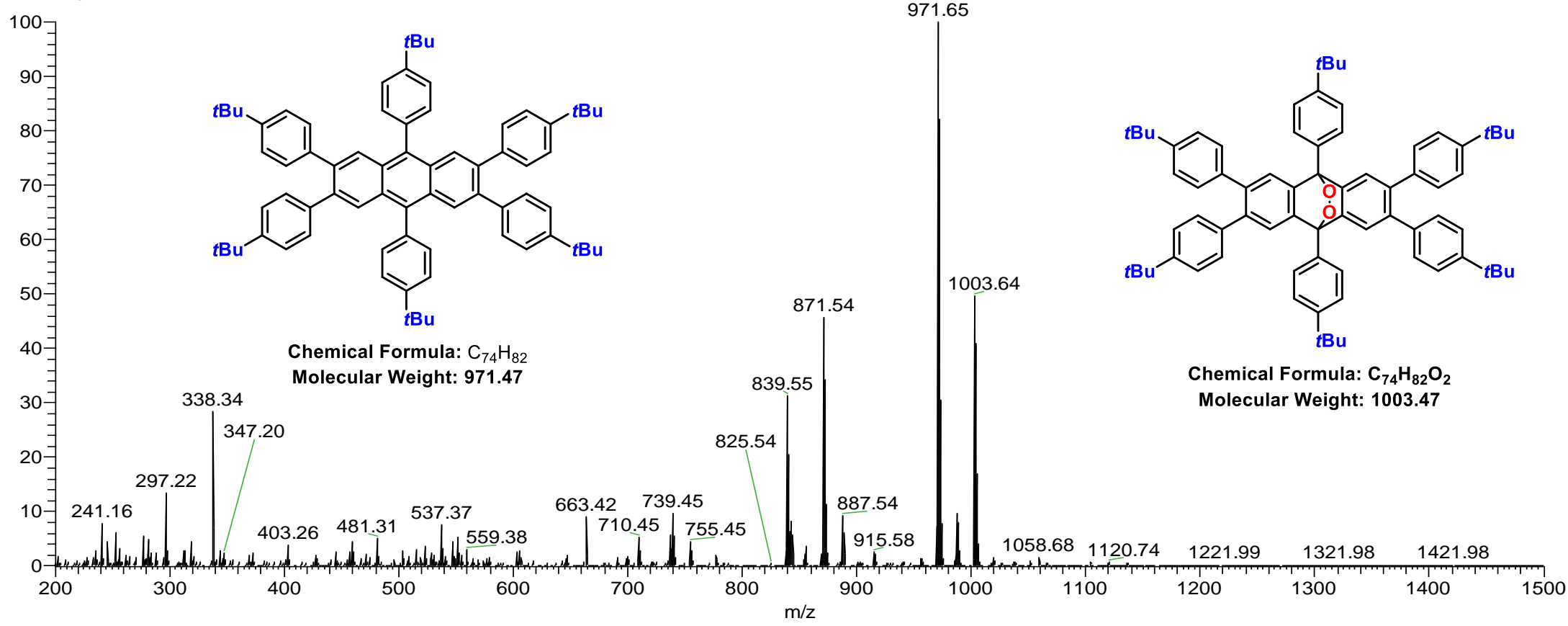

Figure S48 the HRMS spectra of 2,3,6,7,9,10-hexa-(4-tert-butyl-phenyl) anthracene 2a under UV irradiation 720 min. 
fx-73; MW=814; DCTB

$\tan 170216 \_810(0.331) \mathrm{Cn}(\mathrm{Cen}, 4,90.00, \mathrm{Ar}) ; \mathrm{Sb}(15,10.00) ; \mathrm{Sm}(\mathrm{SG}, 2 \times 3.00) ; \mathrm{Cm}(8: 10)$

814.3319

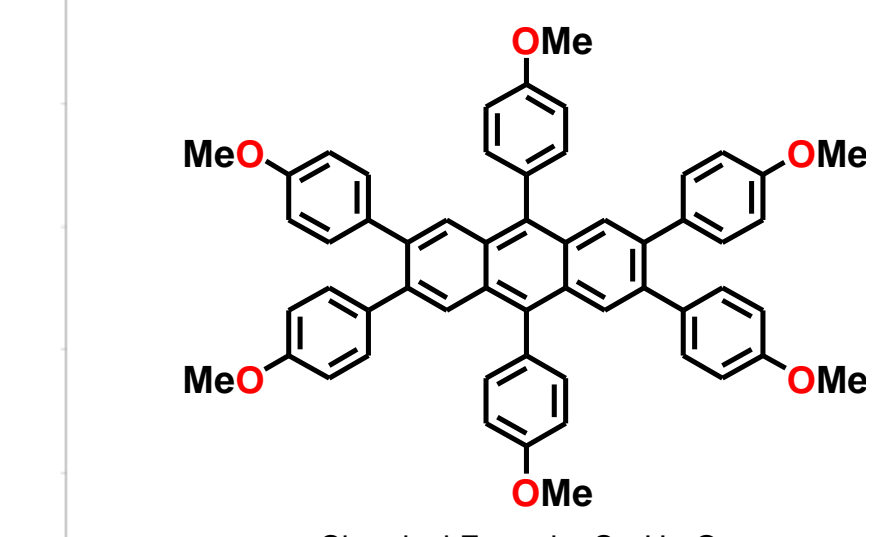

Chemical Formula: $\mathrm{C}_{56} \mathrm{H}_{46} \mathrm{O}_{6}$

$\therefore$

Molecular Weight: 814.98

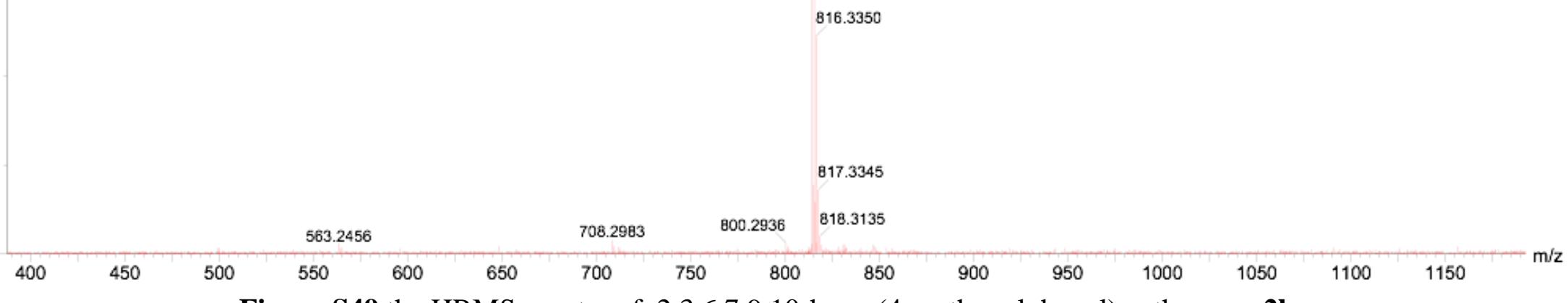

Figure S49 the HRMS spectra of 2,3,6,7,9,10-hexa-(4-methoxylphenyl) anthracene $\mathbf{2 b}$ 
fx-76; $M W=814 / 1628 ;$ DCTB

$\tan 170216 \_76$ (0.199) Cn (Cen,4, 90.00, Ar); Sb (15,10.00); Sm (SG, 2x3.00); Cm (2:6)

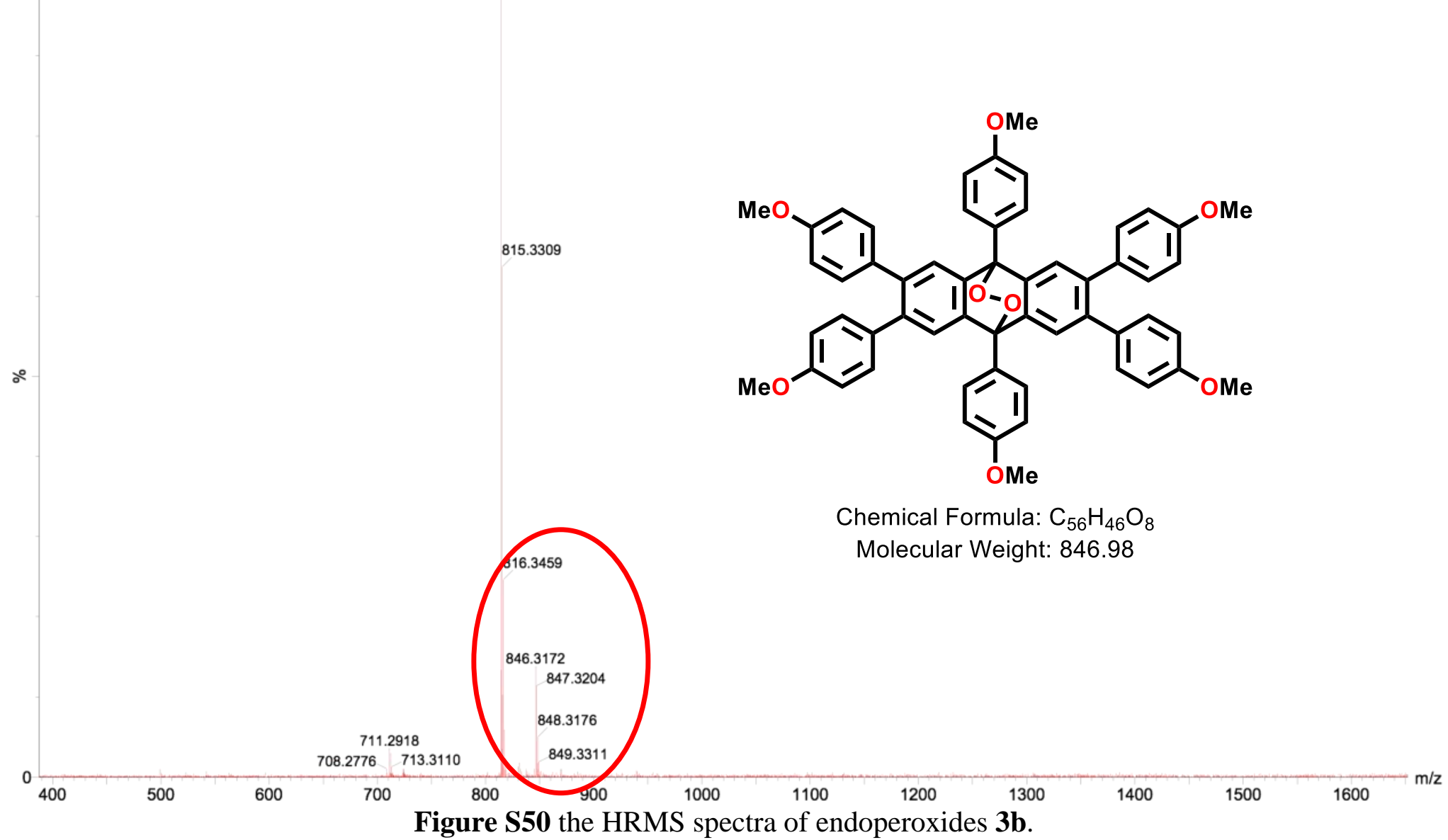


wpf-FX-4; MW=724; DCTB

tan181130_2 4 (0.133) Cn (Cen,2, 10.00, Ar); Sb (5,5.00); Sm (SG, 2x3.00); Cm (4:11)

740.2774

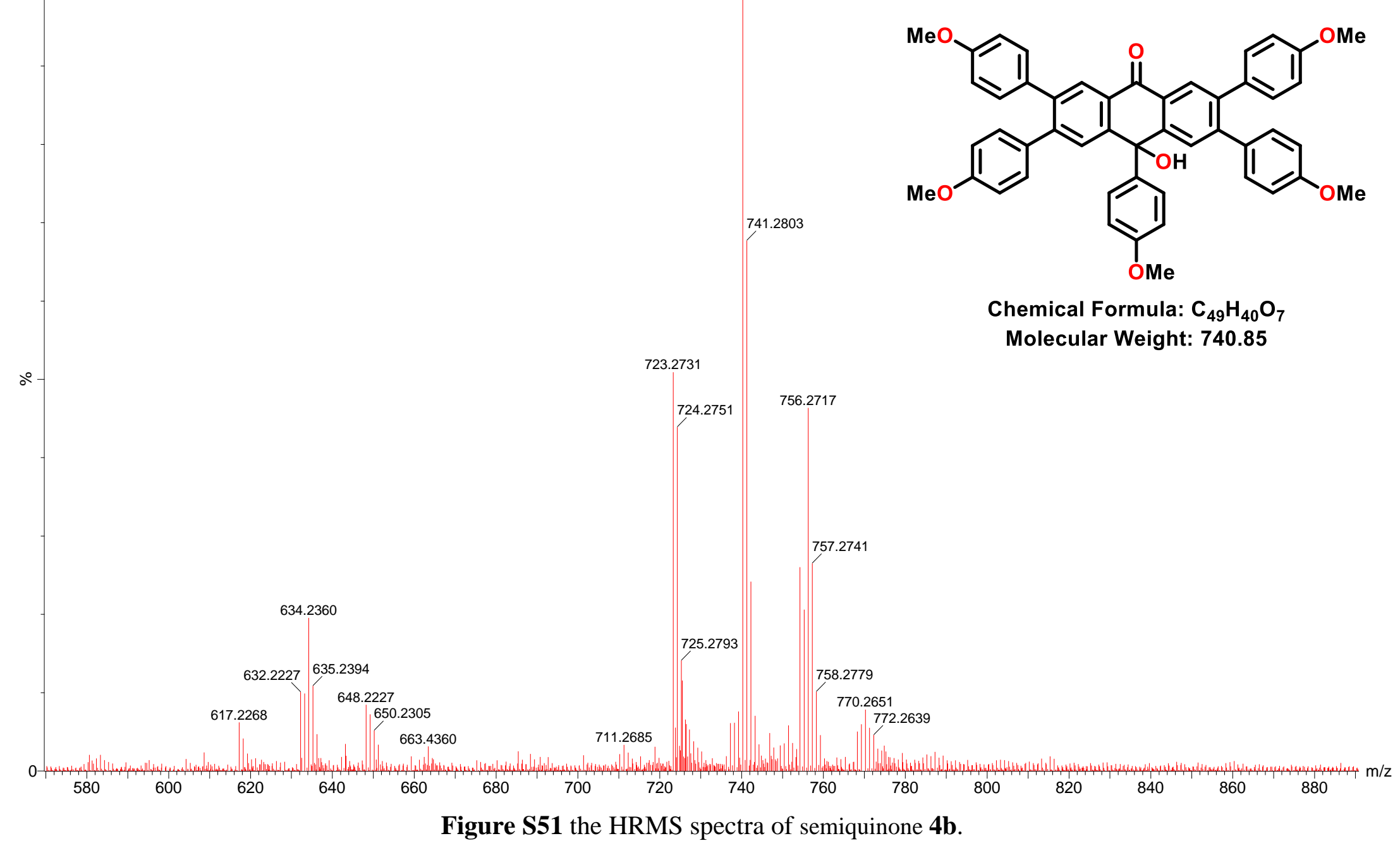


wpf-FX-4; MW=724; DCTB

$\tan 181130 \_410(0.333) \mathrm{Cn}(\mathrm{Cen}, 2,10.00, \mathrm{Ar})$; Sb (5,5.00); Sm (SG, 2×3.00)

100

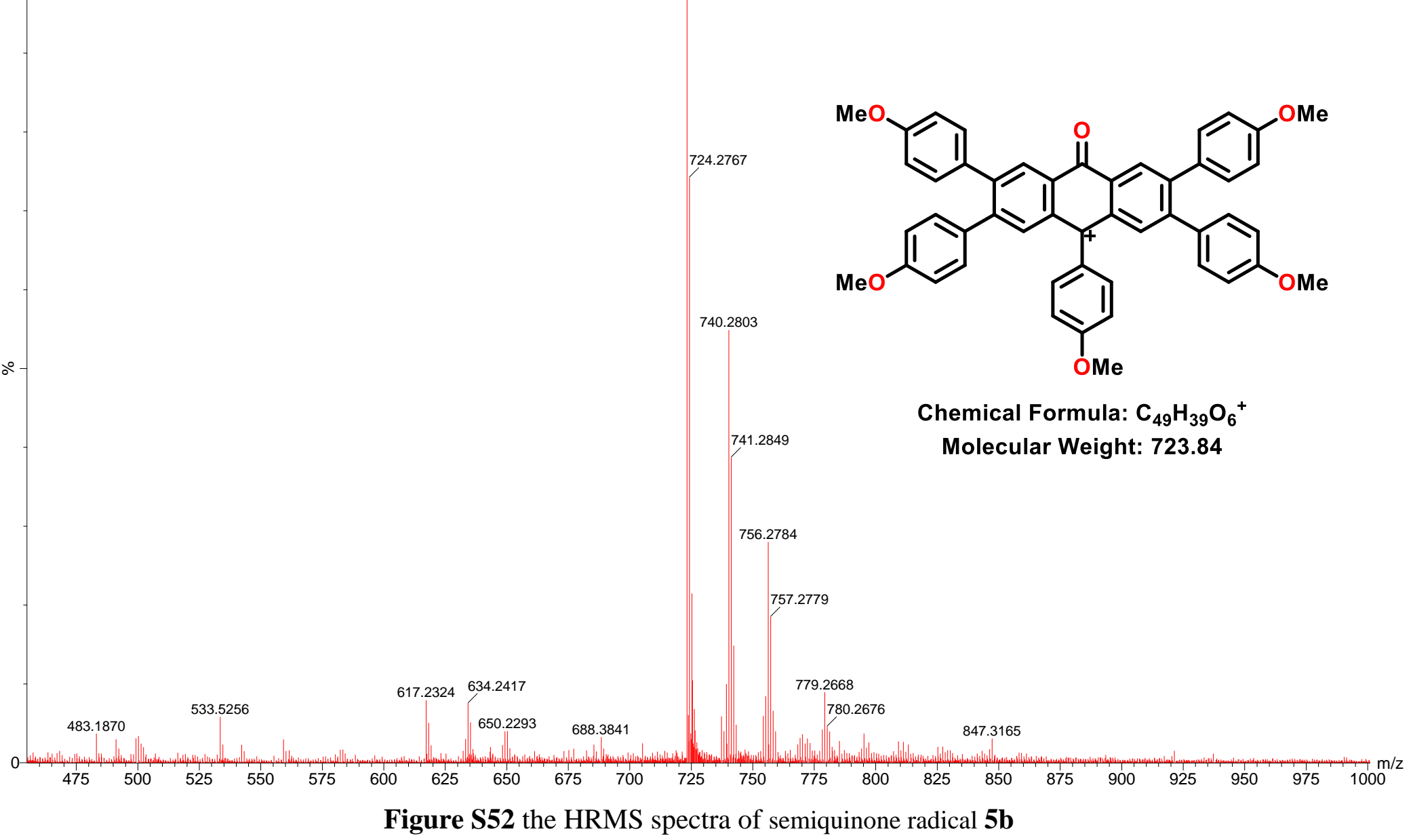

Figure S52 the HRMS spectra of semiquinone radical $\mathbf{5 b}$ 


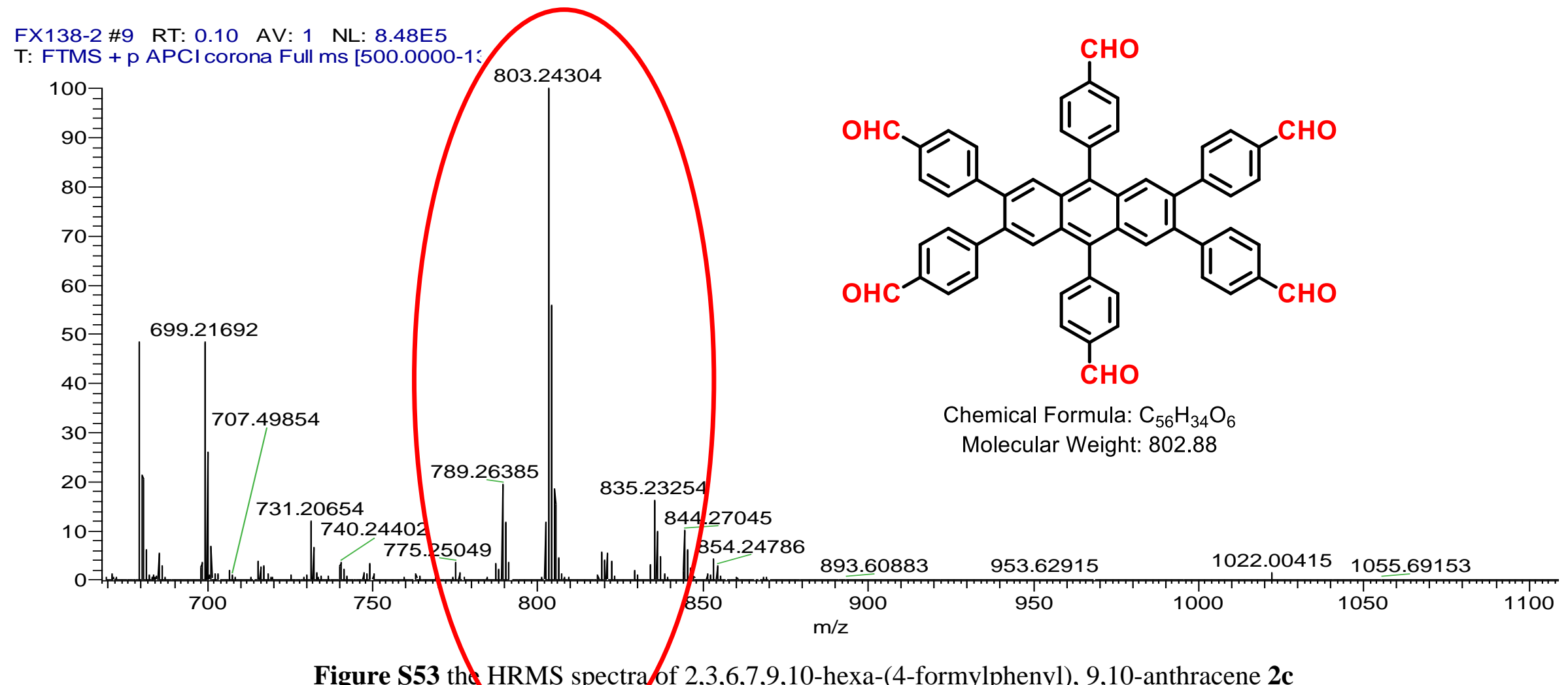

Figure S53 thd HRMS spectra of 2,3,6,7,9,10-hexa-(4-formylphenyl), 9,10-anthracene 2c 
FX-8_20180523033924 \#27 RT: 0.26 AV: 1 NL: $1.34 E 6$

$\mathrm{T}:$ + C APCIQ1MS [400.000-1000.000]

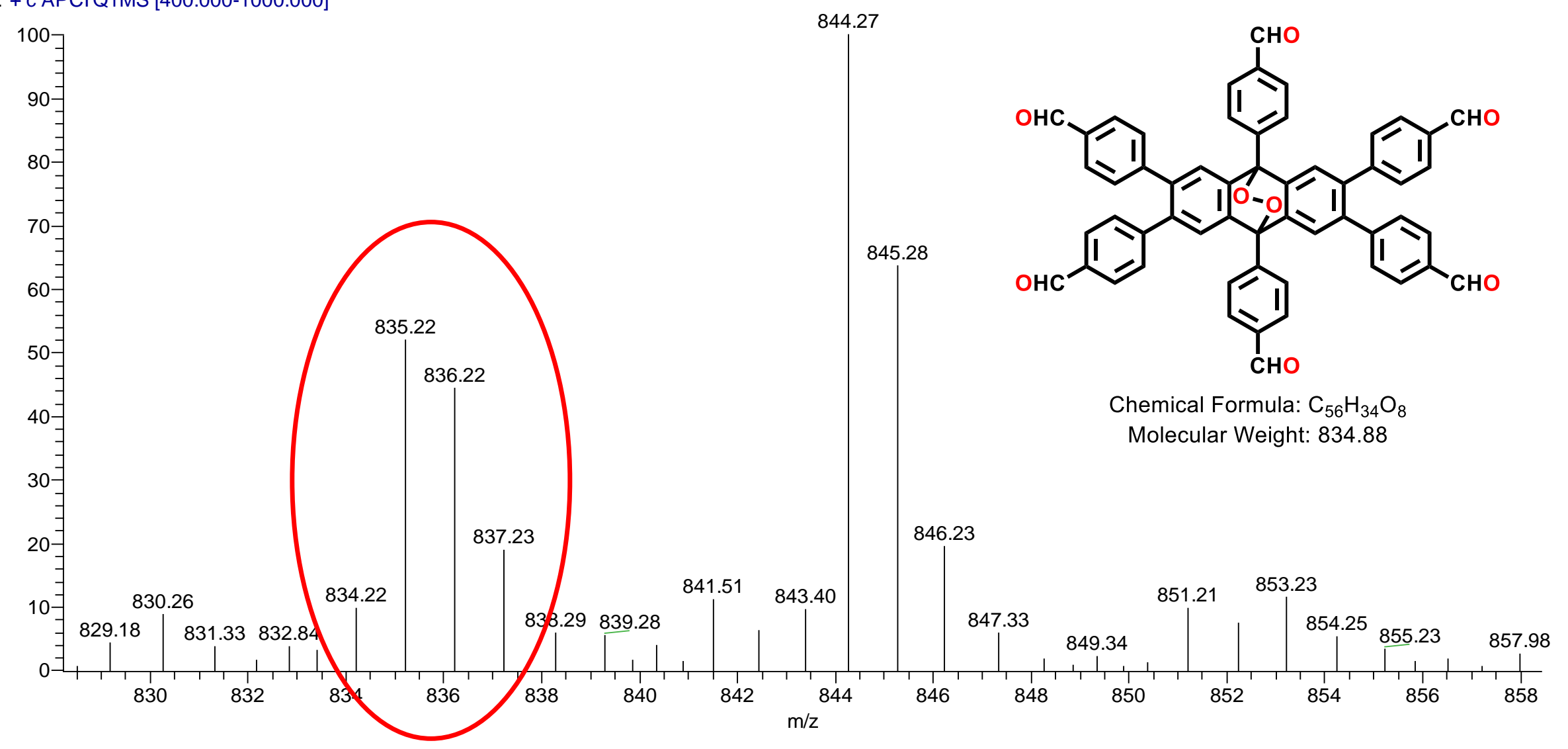

Figure S54 the HRMS spectra of endoperoxides 3c 
CHO-1 \#7-10 RT: $0.07-0.09$ AV: 2 SB: 1 0.05 NL: 1.87E6
T: FTMS + p APCI corona Full ms [200.0000-1500.

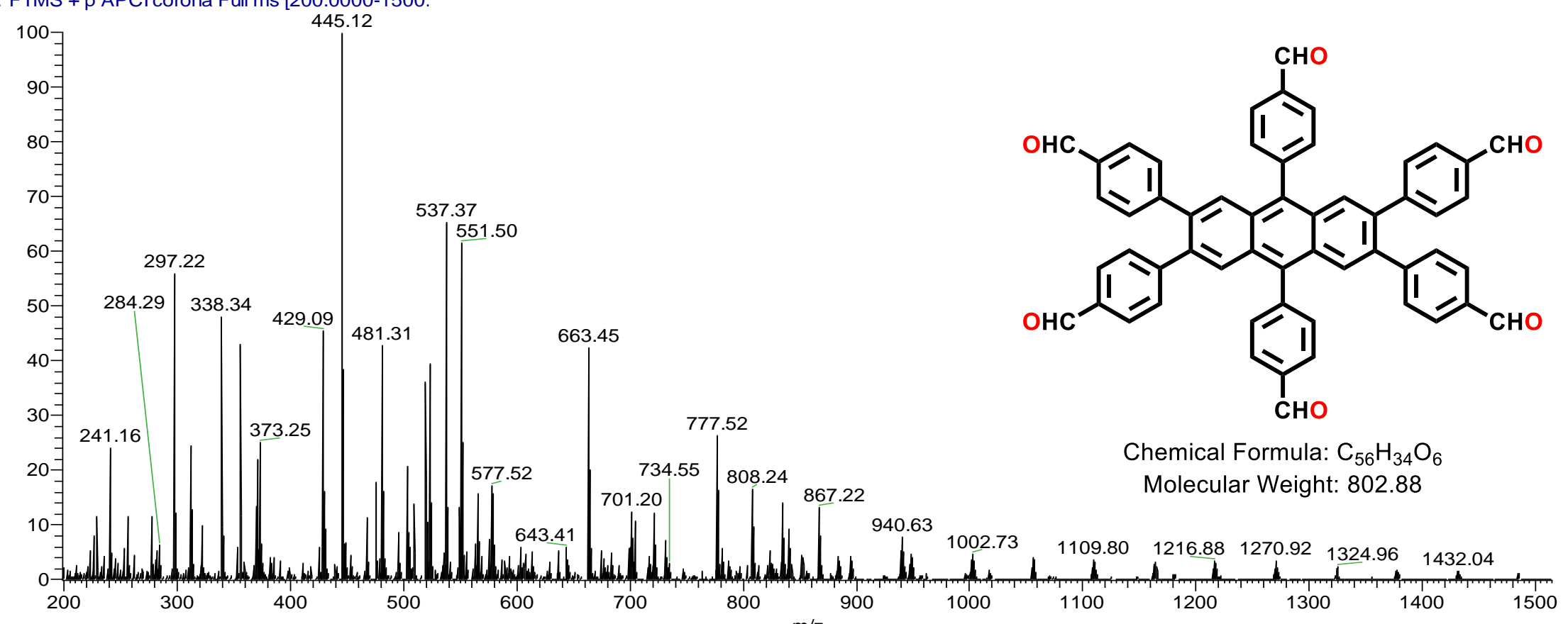

Figure S55 the HRMS spectra of 2c under UV irradiation $360 \mathrm{~min}$. 
CHO-2 \#7 RT: 0.07 AV: 1 SB: 10.05 NL: 1.46E6

T: FTMS + p APCI corona Full ms [200.0000-1500.

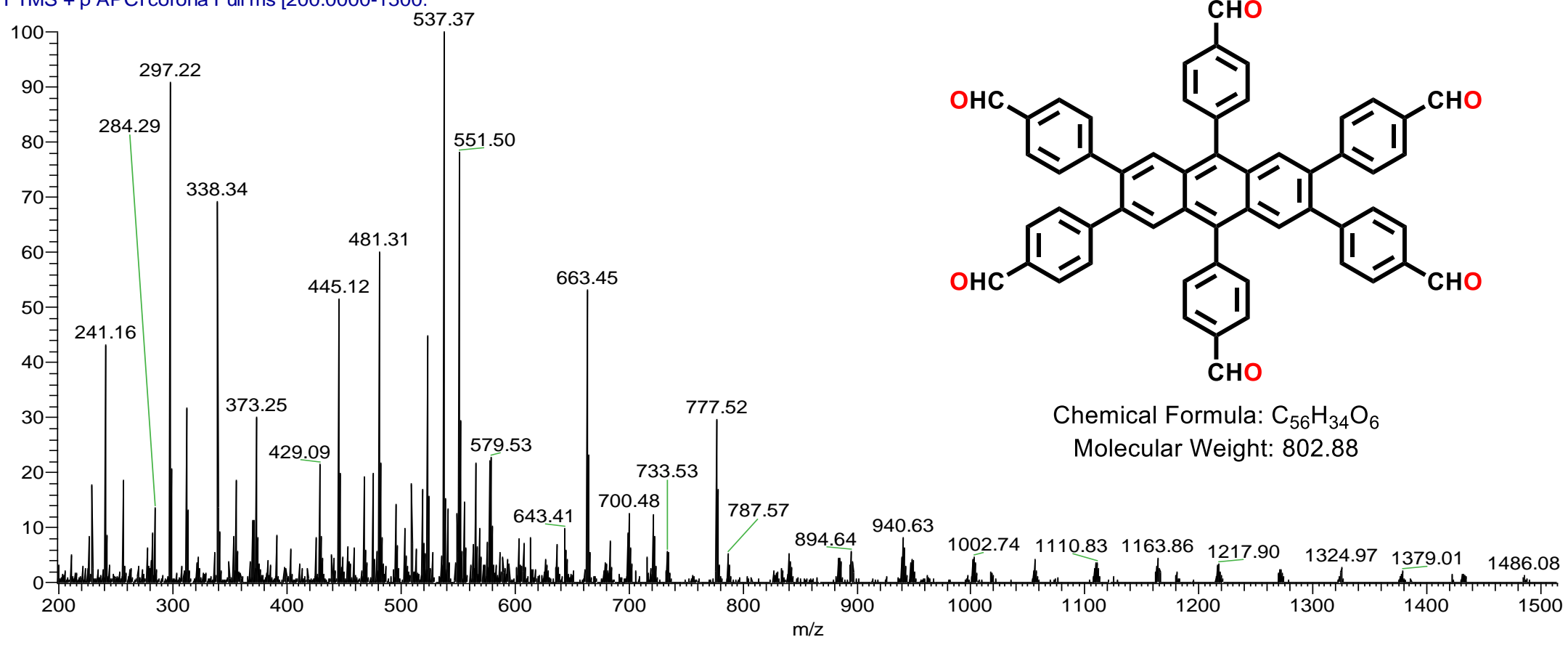

Figure S56 the HRMS spectra of 2c under UV irradiation $720 \mathrm{~min}$. 
FX101 \#10 RT: 0.10 AV: 1 NL: 1.04E8

T: FTMS - p APCl corona Full ms [150.0000-20

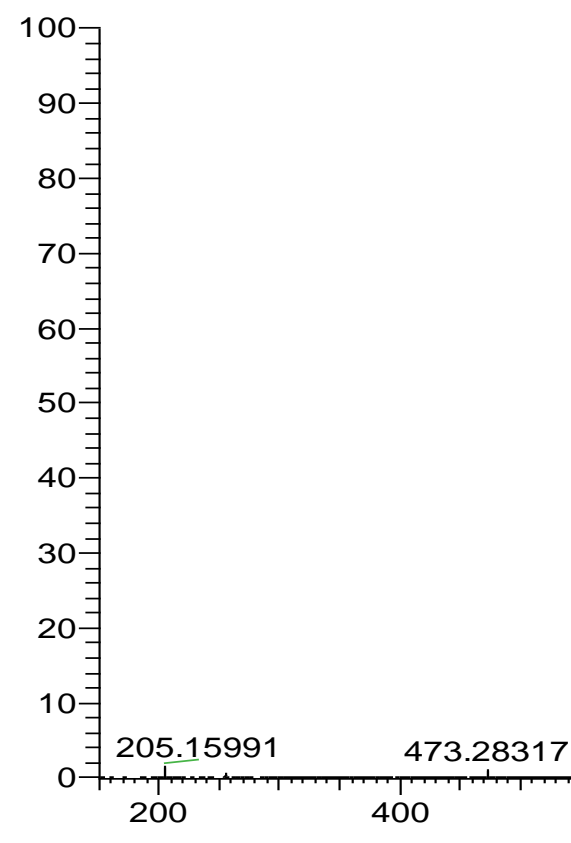

20

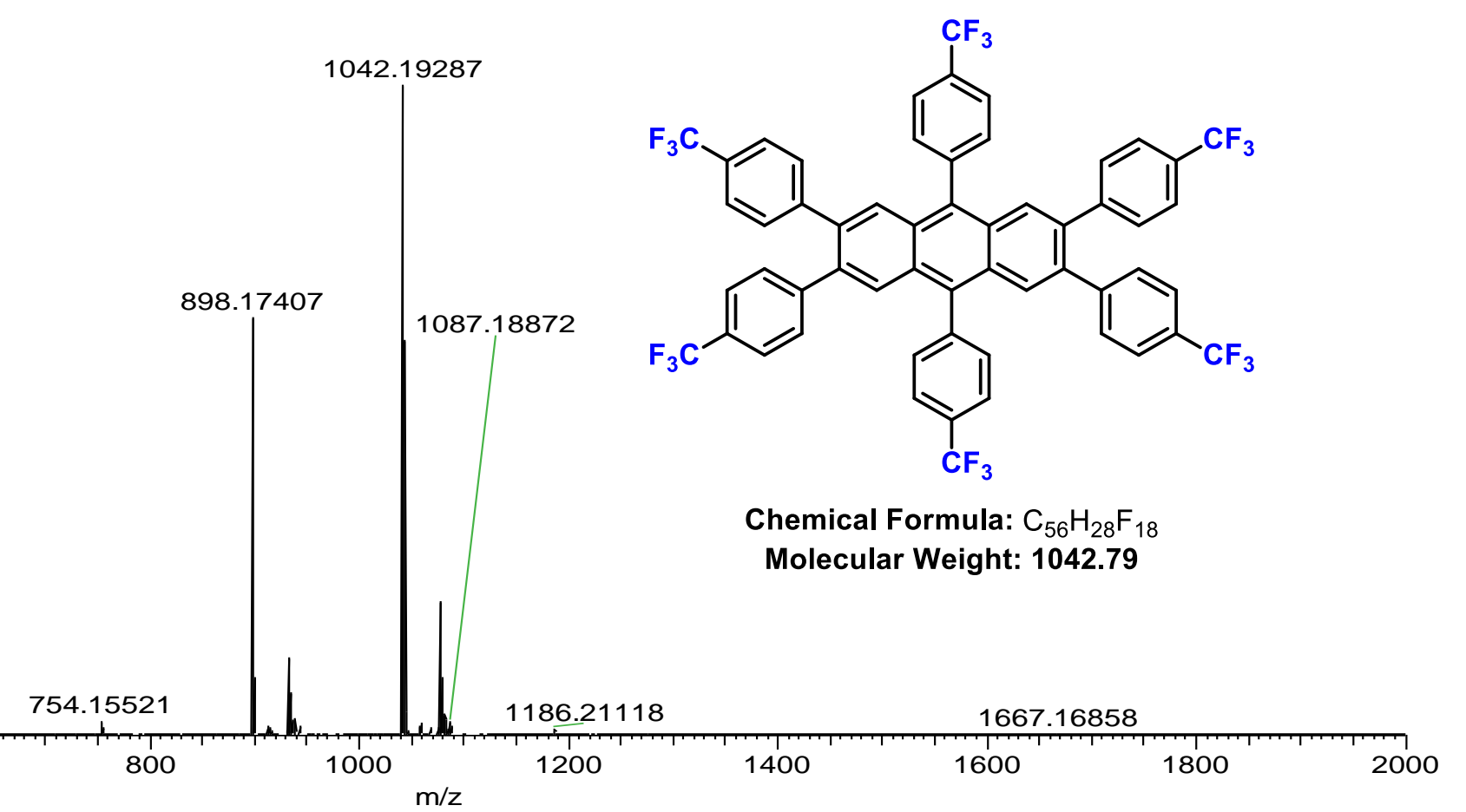

Figure S57 the HRMS spectra of 2d. 
FX-109 \#6 RT: 0.07 AV: 1 NL: 6.61E5

T: FTMS - p APCl corona Full ms [150.0000-1500.0000]

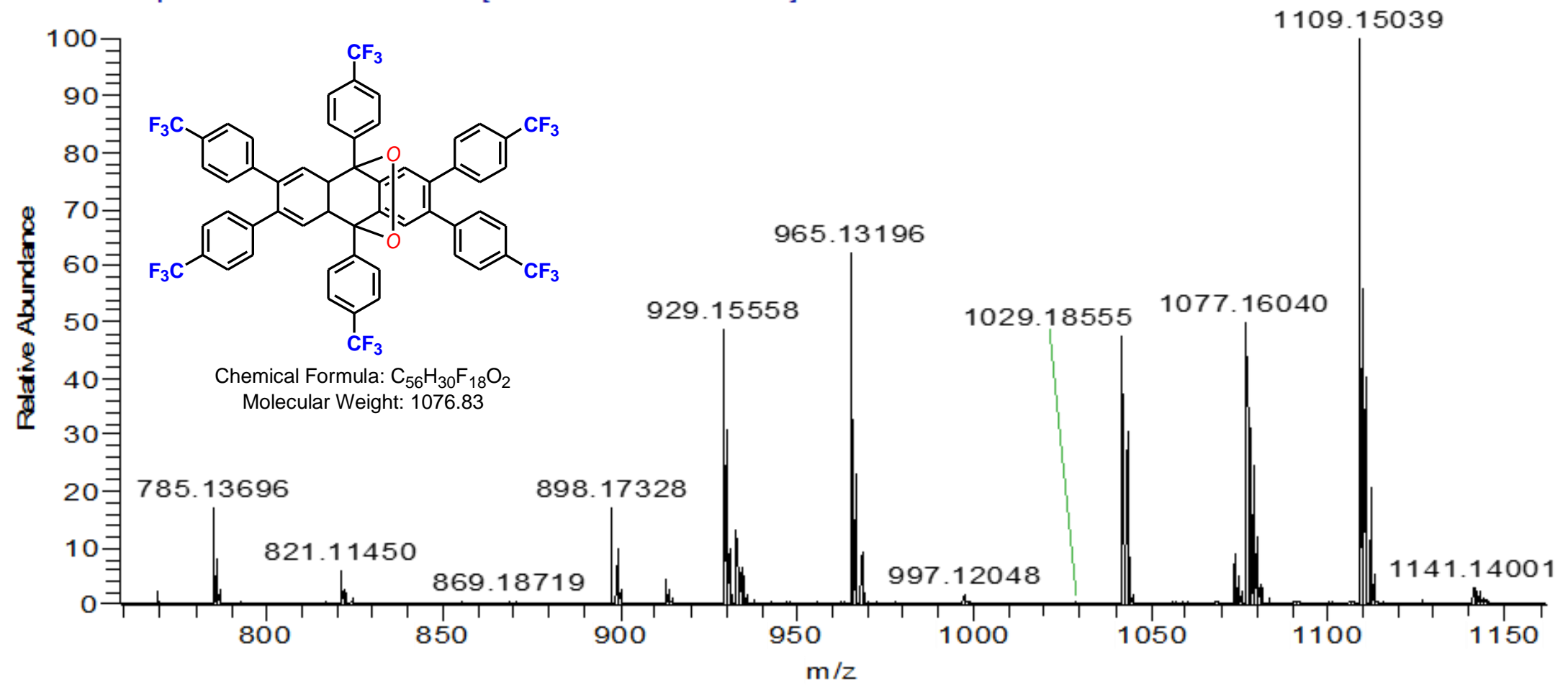

Figure S58 the HRMS spectra of $\mathbf{2 d}$ under UV irradiation $720 \mathrm{~min}$. 
FX-CF3_20191226144402\#10 RT: 0.10 AV: 1 SB: 10.04 NL: 3.23E7

T: FTMS - p APCI corona Full ms [200.0000-1500.C

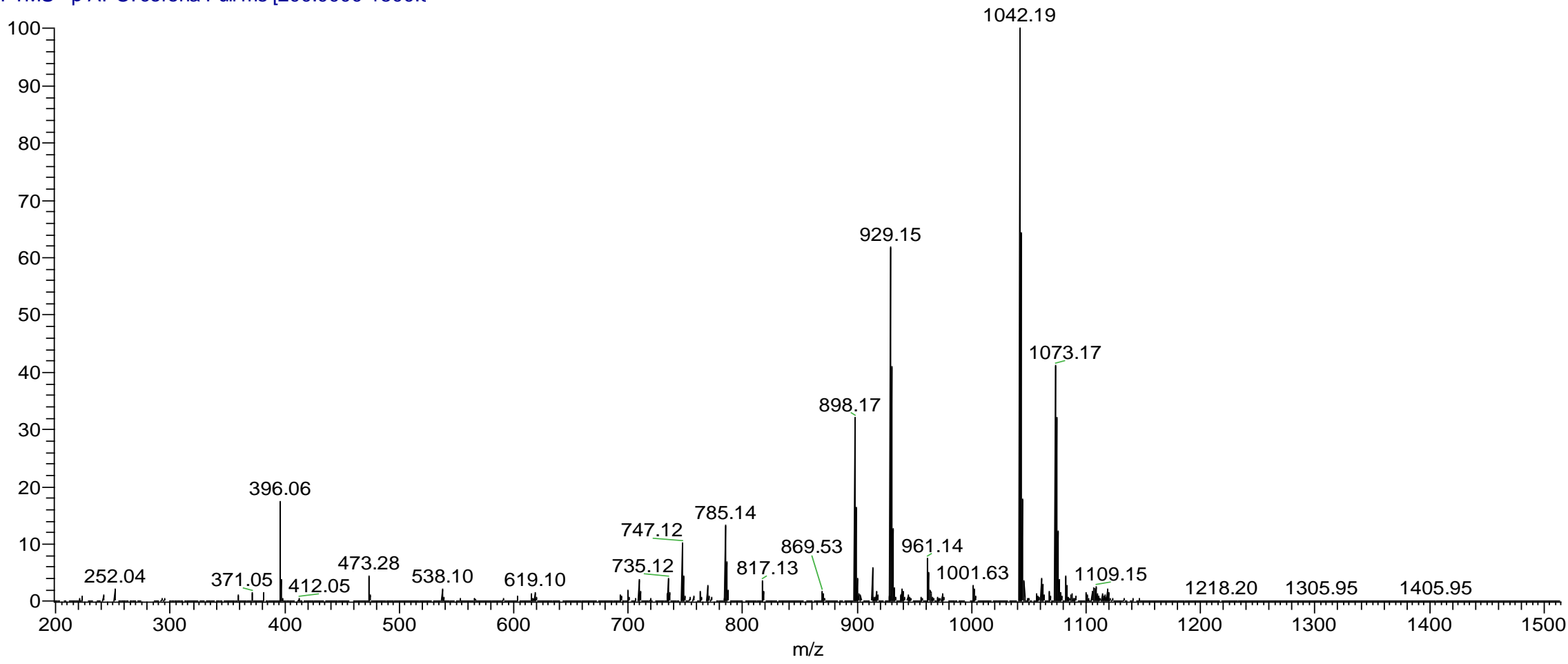

Figure S59 the HRMS spectra of 2d under UV irradiation 360 min. 
CF3-2 \#7 RT: $0.07 \quad$ AV: 1 NL: 1.99E8

T: FTMS + p APCI corona Full ms [200.0000-1500

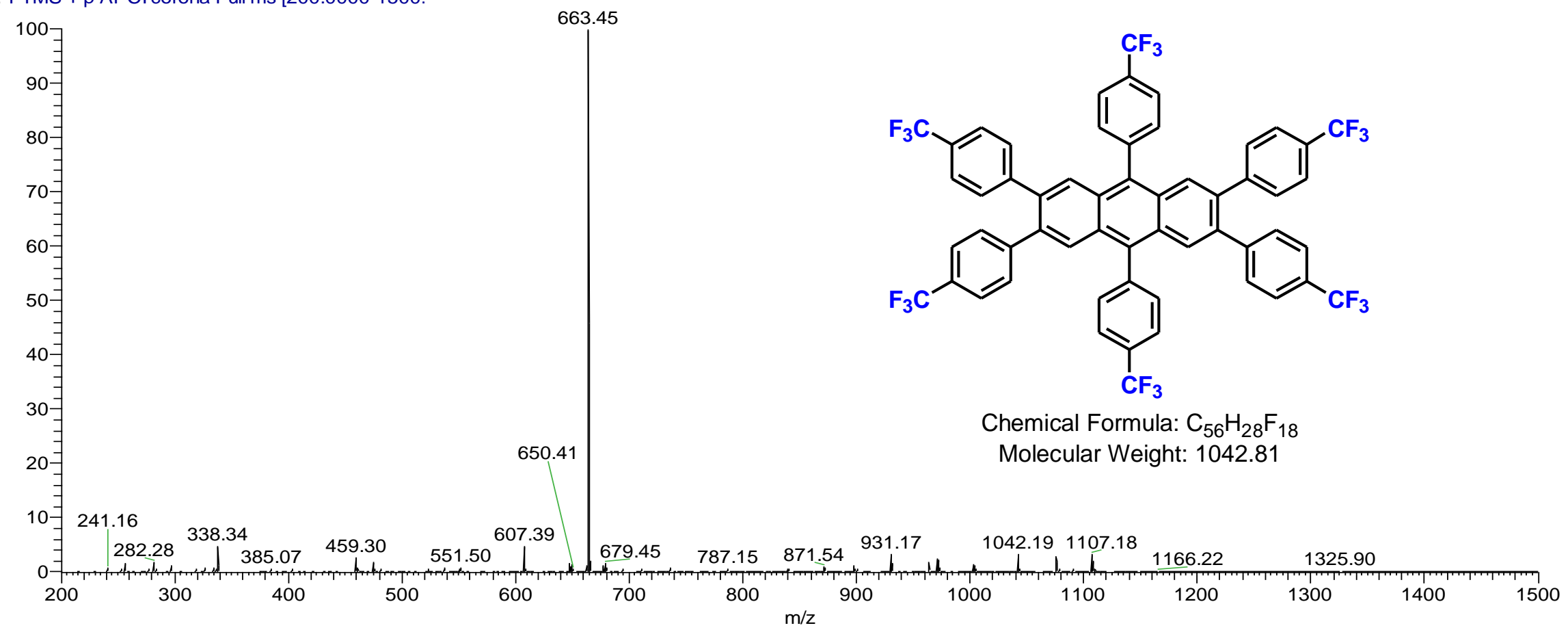

Figure S60 the HRMS spectra of $\mathbf{2 d}$ under UV irradiation $720 \mathrm{~min}$. 
FX-64 \#9 RT: $0.09 \quad$ AV: 1 NL: $4.02 E 7$

T: FTMS + p APCl corona Full ms [100.0000-1200

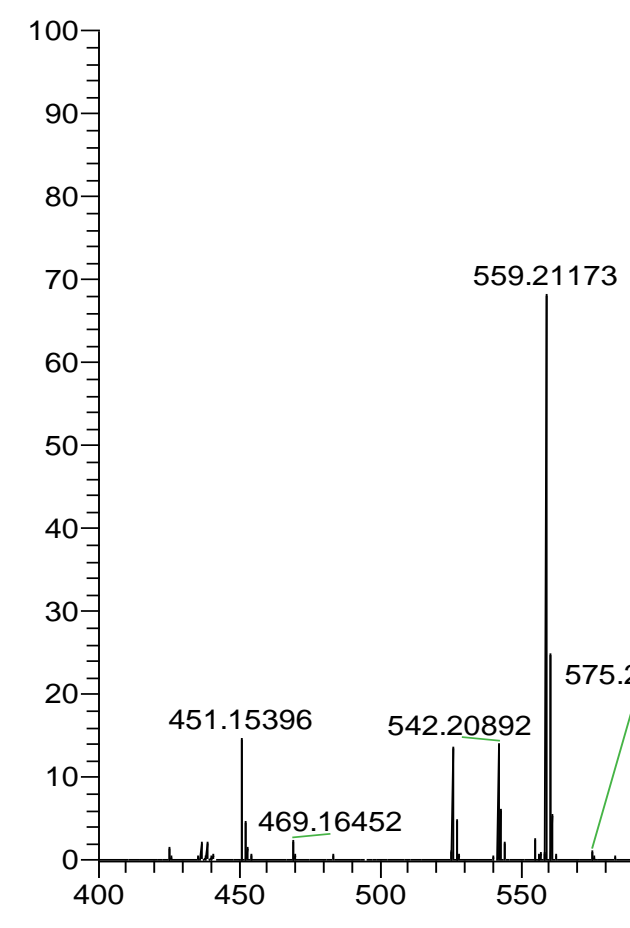

Figure S61 the HRMS spectra of the products of thermolysis of EPO $3 \mathbf{b}$ at $140{ }^{\circ} \mathbf{C}$.
847.32666

831.33173

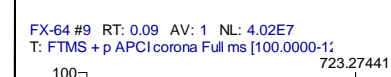

\section{8} 663.45398 617.23242

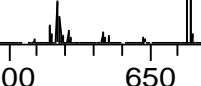

650

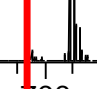

(1) 
Table S1 The possible products of thermolysis of EPO $3 \mathbf{b}$ at $140 \mathrm{oC}$

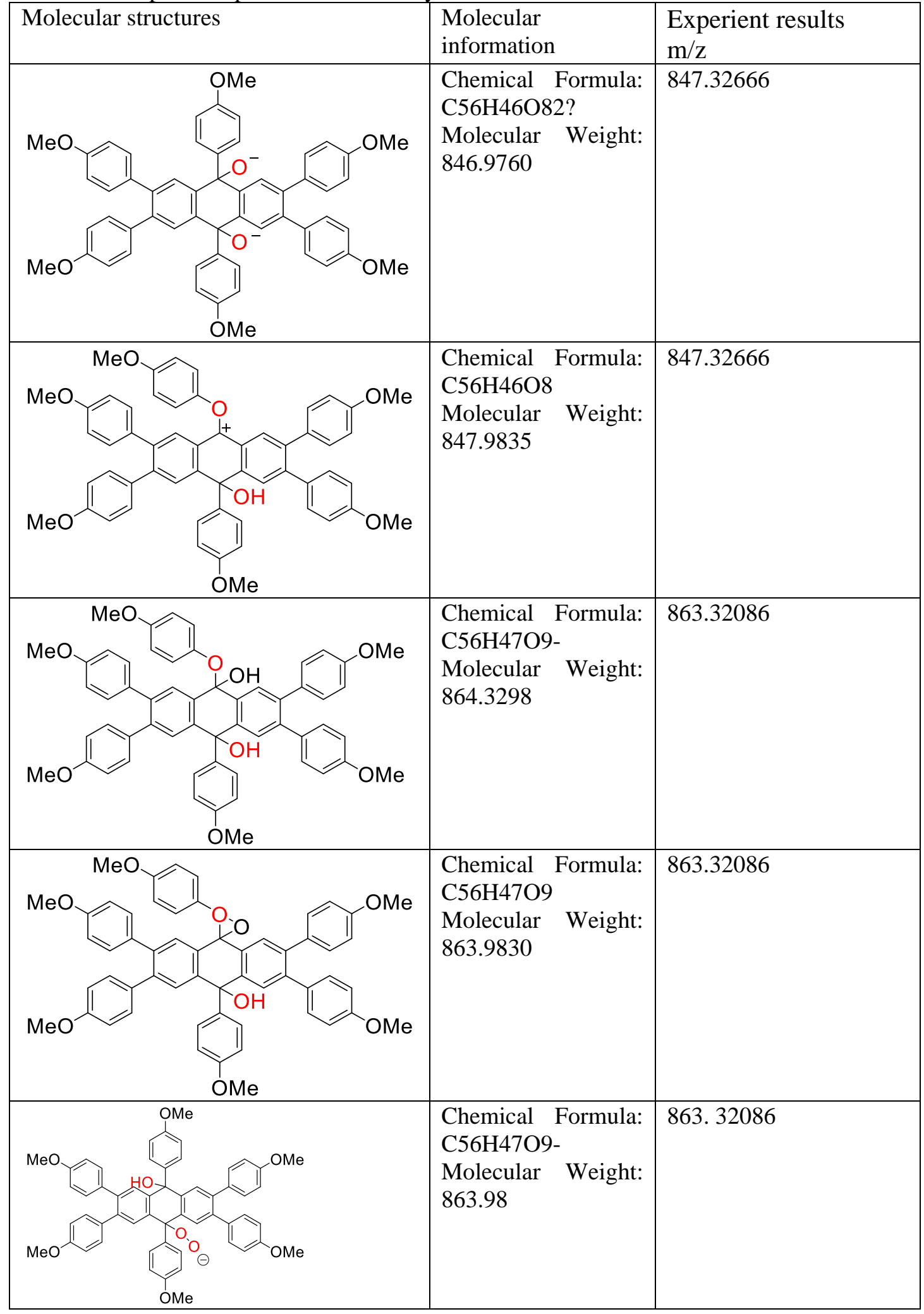



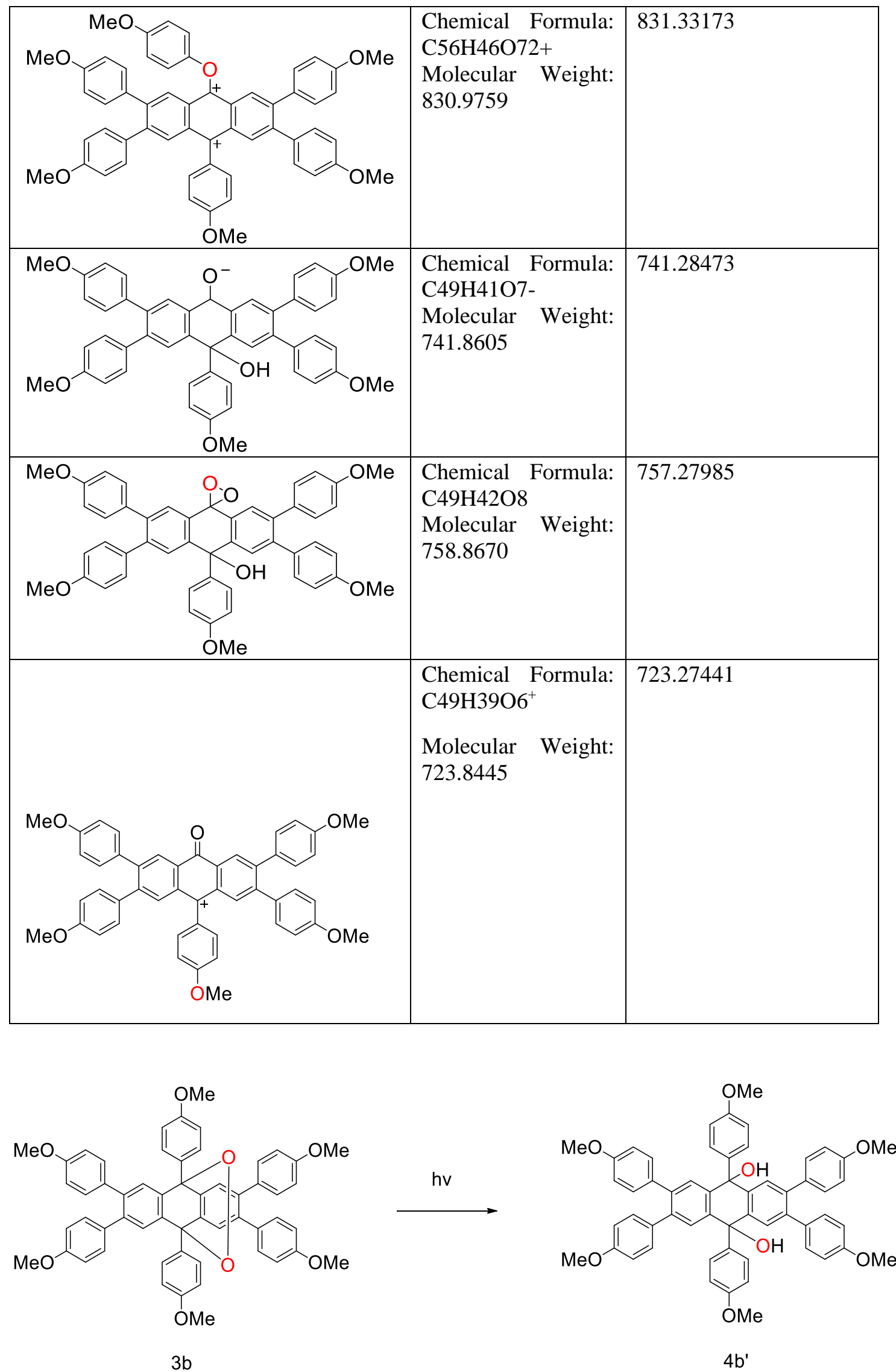

Scheme S1 A controllable photo-induced photochemical reaction from hexa-aryl substituted anthracenes $\mathbf{2 b}$ to $\mathbf{4 b}$ ' under UV-irradiation $\left(\lambda_{\mathrm{ex}}=365 \mathrm{~nm}, 100 \mathrm{~W}\right)$. 
Type III

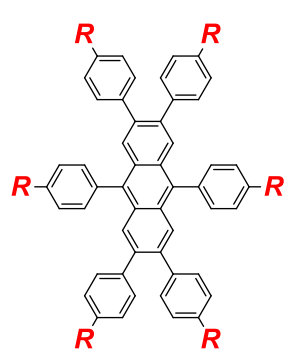

$\left[\mathrm{O}_{2}\right]$
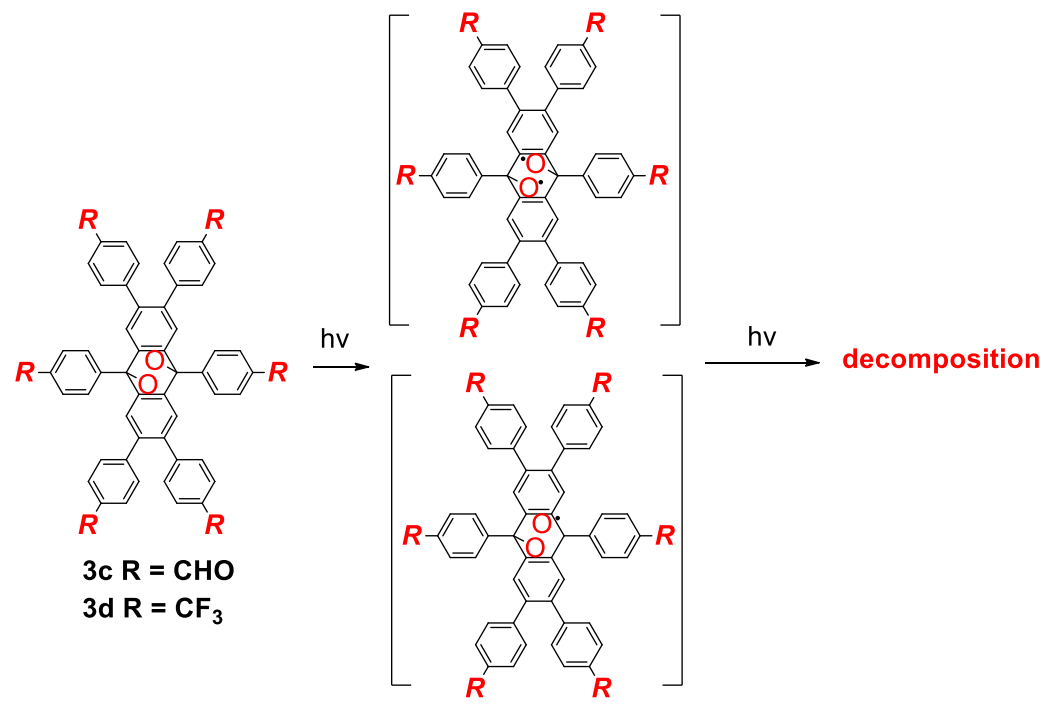

intermediation

Scheme S2 A controllable photo-induced photochemical reaction from hexa-aryl substituted anthracenes $\mathbf{2 c}$ and $\mathbf{2 d}$ to decomposition under UV-irradiation $\left(\lambda_{\mathrm{ex}}=365\right.$ nm, 100W). 


\section{X-ray crystallography}

Table S2 Summary of crystal data for hexa-aryl anthracenes

\begin{tabular}{|c|c|c|c|c|c|}
\hline Parameter & $\mathbf{2 a}$ & $\mathbf{2 b}$ & $2 b^{\prime}$ & $\mathbf{3 b}$ & $4 b$ \\
\hline Empirical formula & $\mathrm{C}_{74} \mathrm{H}_{82}$ & $\mathrm{C}_{56} \mathrm{H}_{46} \mathrm{O}_{6}$ & $\mathrm{C}_{62} \mathrm{H}_{60} \mathrm{O}_{6}$ & $\mathrm{C}_{56} \mathrm{H}_{46} \mathrm{O}_{8} \cdot \mathrm{H}_{2} \mathrm{O}$ & $\mathrm{C}_{49} \mathrm{H}_{40} \mathrm{O}_{7}$ \\
\hline CCDC Number & 2052694 & 1844506 & 1844505 & 2052692 & 2052693 \\
\hline $\begin{array}{l}\text { Formula weight } \\
{\left[\mathrm{g} \mathrm{mol}^{-1}\right]}\end{array}$ & 971.39 & 814.93 & 901.10 & 882.96 & 740.81 \\
\hline Crystal system & monoclinic & orthorhombic & monoclinic & Monoclinic & monoclinic \\
\hline Space group & $P 12 / n 1$ & Pbcn & $P 2{ }_{1} / c$ & $\mathrm{C} 2 / \mathrm{c}$ & $P 12 / n 1$ \\
\hline$A[\AA ̊ \AA]$ & $23.2737(7)$ & $15.9153(9)$ & $5.8920(4)$ & $25.5338(6)$ & $9.2242(3)$ \\
\hline$B[\AA]$ & $6.3735(2)$ & $26.2137(9)$ & $16.2457(11)$ & $9.1535(2)$ & $28.8242(10)$ \\
\hline$C[\AA]$ & $23.7760(8)$ & $10.0287(9)$ & $25.139(3)$ & $22.2922(6)$ & $15.0769(4)$ \\
\hline $\boldsymbol{\alpha}\left[{ }^{\circ}\right]$ & -- & -- & -- & -- & -- \\
\hline$\beta\left[^{\circ}\right]$ & $119.0880(10)$ & & $94.700(8)$ & $113.752(2)$ & $106.7980(10)$ \\
\hline$\gamma\left[^{\circ}\right]$ & -- & -- & -- & -- & -- \\
\hline Volume $\left[\AA^{3}\right]$ & $3081.99(17)$ & $4184.0(5)$ & 2398.2(3) & $4768.9(2)$ & $3837.6(2)$ \\
\hline $\mathbf{Z}$ & 2 & 8 & 4 & 4 & 4 \\
\hline Density, calcd $\left[\mathrm{g} \mathrm{m}^{-3}\right]$ & 1.047 & 1.248 & 1.248 & 1.230 & 1.282 \\
\hline Temperature [K] & $225(2)$ & $99.97(11)$ & $99.94(15)$ & $293(2)$ & $230(2)$ \\
\hline Unique reflns & 26234 & 12204 & 12208 & 9085 & 25176 \\
\hline Obsdreflns & 4808 & 3704 & 4159 & 4660 & 10478 \\
\hline Parameters & 413 & 283 & 311 & 289 & 511 \\
\hline$R_{\text {int }}$ & 0.1244 & 0.0357 & 0.0913 & 0.0901 & 0.0892 \\
\hline $\mathrm{R}[\mathrm{I}>\mathbf{2 \sigma}(\mathrm{I})]^{\mathrm{a}}$ & 0.2669 & 0.0664 & 0.1079 & 0.0753 & 0.2293 \\
\hline $\mathrm{wR}[\mathrm{I}>\mathbf{2 \sigma}(\mathrm{I})]^{\mathrm{b}}$ & 0.2933 & 0.1633 & 0.2667 & 0.2211 & 0.2820 \\
\hline GOF on F2 & 1.098 & 1.007 & 1.008 & 1.005 & 1.029 \\
\hline
\end{tabular}

a Conventional $R$ on $\mathrm{F}_{\mathrm{hkl}}: \Sigma|| \mathrm{F}_{\mathrm{o}}|-| \mathrm{F}_{\mathrm{c}}|| / \sigma\left|\mathrm{F}_{\mathrm{o}}\right|$. ${ }^{\mathrm{b}}$ Weighted $R$ on $\left|\mathrm{F}_{\mathrm{hkl}}\right|^{2}: \Sigma\left[\mathrm{w}\left(\mathrm{F}_{\mathrm{o}}{ }^{2}-\right.\right.$ $\left.\left.\mathrm{F}_{\mathrm{c}}^{2}\right)^{2}\right] / \Sigma\left[\mathrm{w}\left(\mathrm{F}_{\mathrm{o}}^{2}\right)^{2}\right]^{1 / 2}$ 
Both crystals crystallized in the monoclinic system, space group is $\mathrm{P} 2 / \mathrm{n}$ for $\mathbf{2 a}$ and $\mathrm{P} 21 / \mathrm{c}$ for $\mathbf{2 b}$, respectively. Noticeable, the aryl group at the 9,10-positions was nearly perpendicular $\left(\mathrm{ca} .87^{\circ}\right)$ to the anthracene core in the crystal, while the other substituents at the 2-, 3-, 6- and 7-positions adopted a twisted conformation with dihedral angles in the range $41-66^{\circ}$. In addition, the anthracene core displayed a non-planar structure with a dihedral angle of $<4^{\circ}$, presumably due to the six substituents at the bay positions. Both crystals $\mathbf{2 a}$ and $\mathbf{2 b}$ also adopted to similar packing mode, and no $\pi-\pi$ stacking was observed in the crystal packing structure, which was attributed to the steric hindrance of the hexa-aryl substituents groups. Both packing structures can be described as a herringbone motif structure with center-to-center distances of 4.39-4.54 $\AA$ connected by several intermolecular C-H $\cdots \pi$ interactions (Figure S62-S73). Furthermore, based on density functional theory (DFT) calculations (at the B3LYP/6-31+G** level), the optimized structures of $\mathbf{2} \mathbf{a}$ and $\mathbf{2} \mathbf{b}$ are in good agreement with their crystal structures (see Figure S70). 


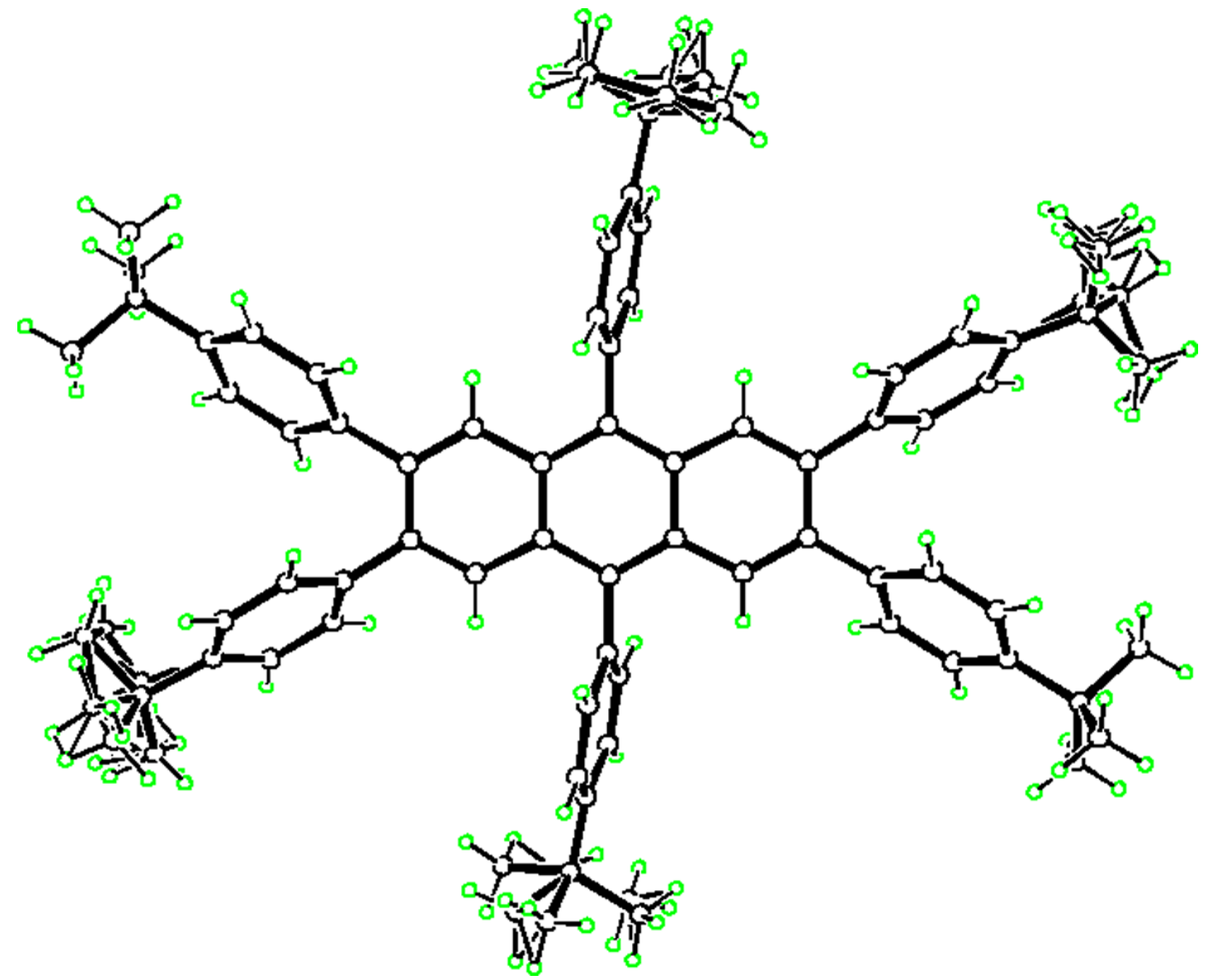

Figure S62 single crystal structures of 2a, in ORTEP drawing with thermal ellipsoids at $30 \%$ probability. 


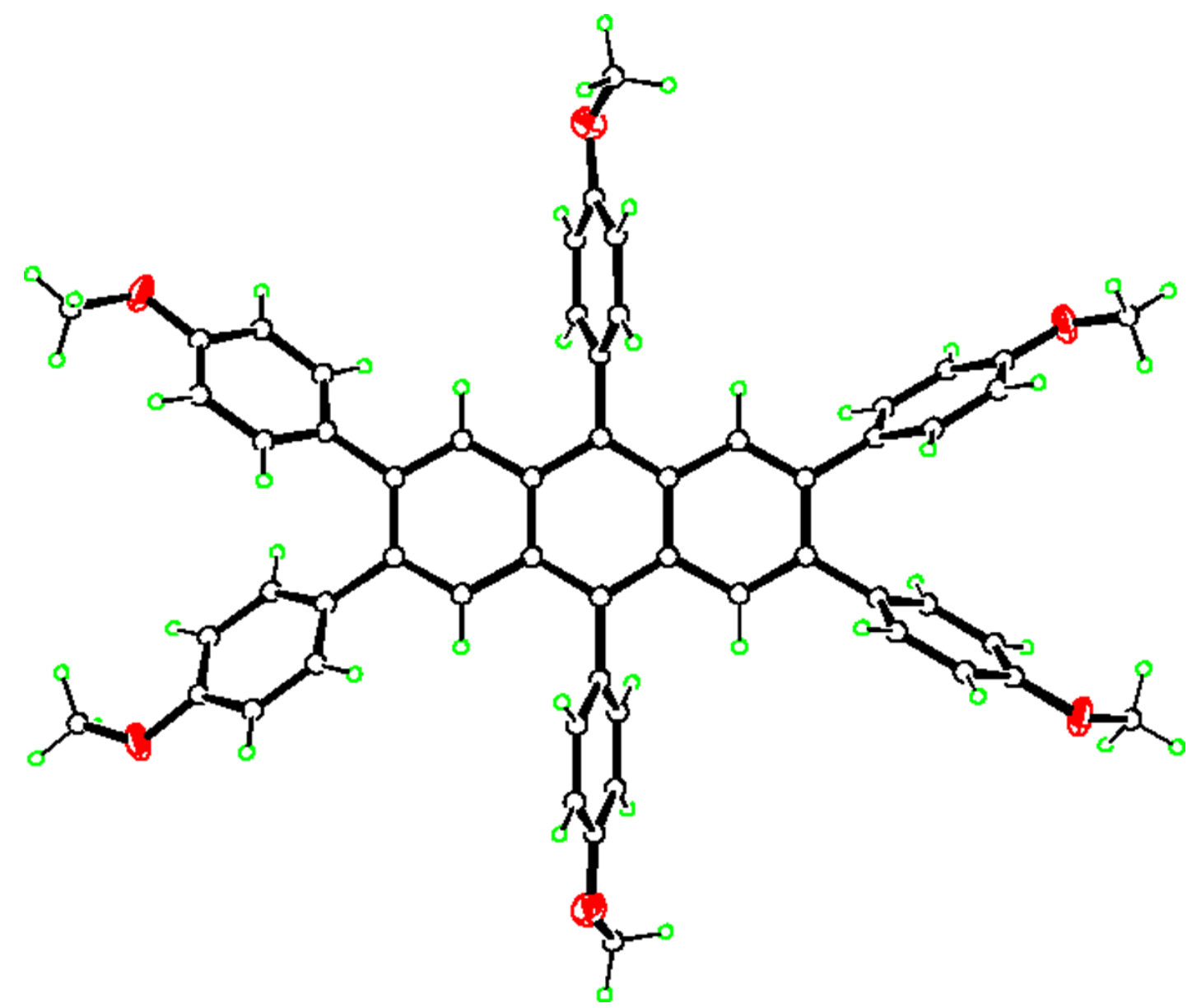

Figure S63 single crystal structures of $\mathbf{2 b}$, in ORTEP drawing with thermal ellipsoids at $30 \%$ probability. 


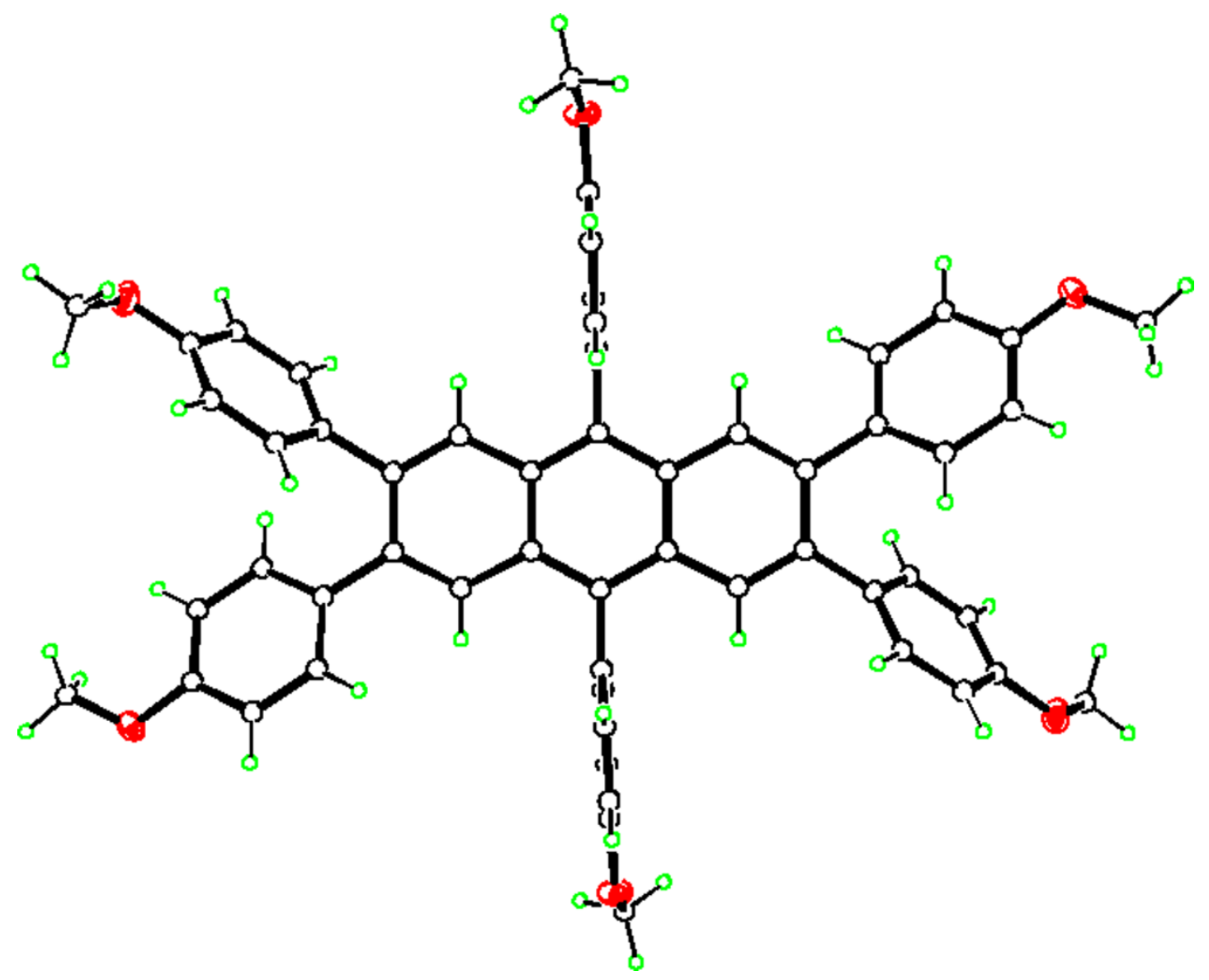

Figure S64 single crystal structures of 2b', in ORTEP drawing with thermal ellipsoids at $30 \%$ probability. 


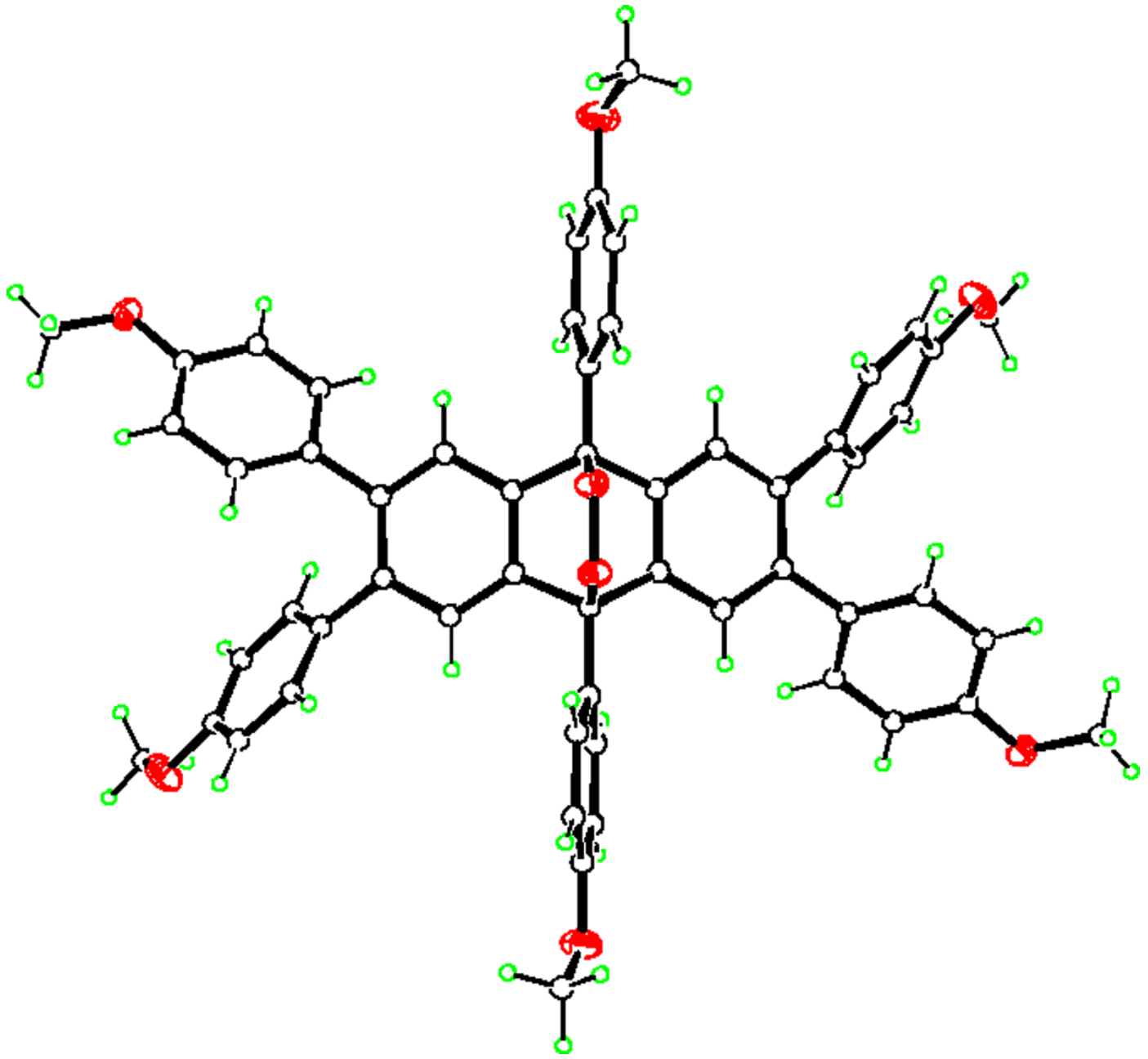

Figure S65 single crystal structures of $\mathbf{3 b}$, in ORTEP drawing with thermal ellipsoids at $30 \%$ probability. 


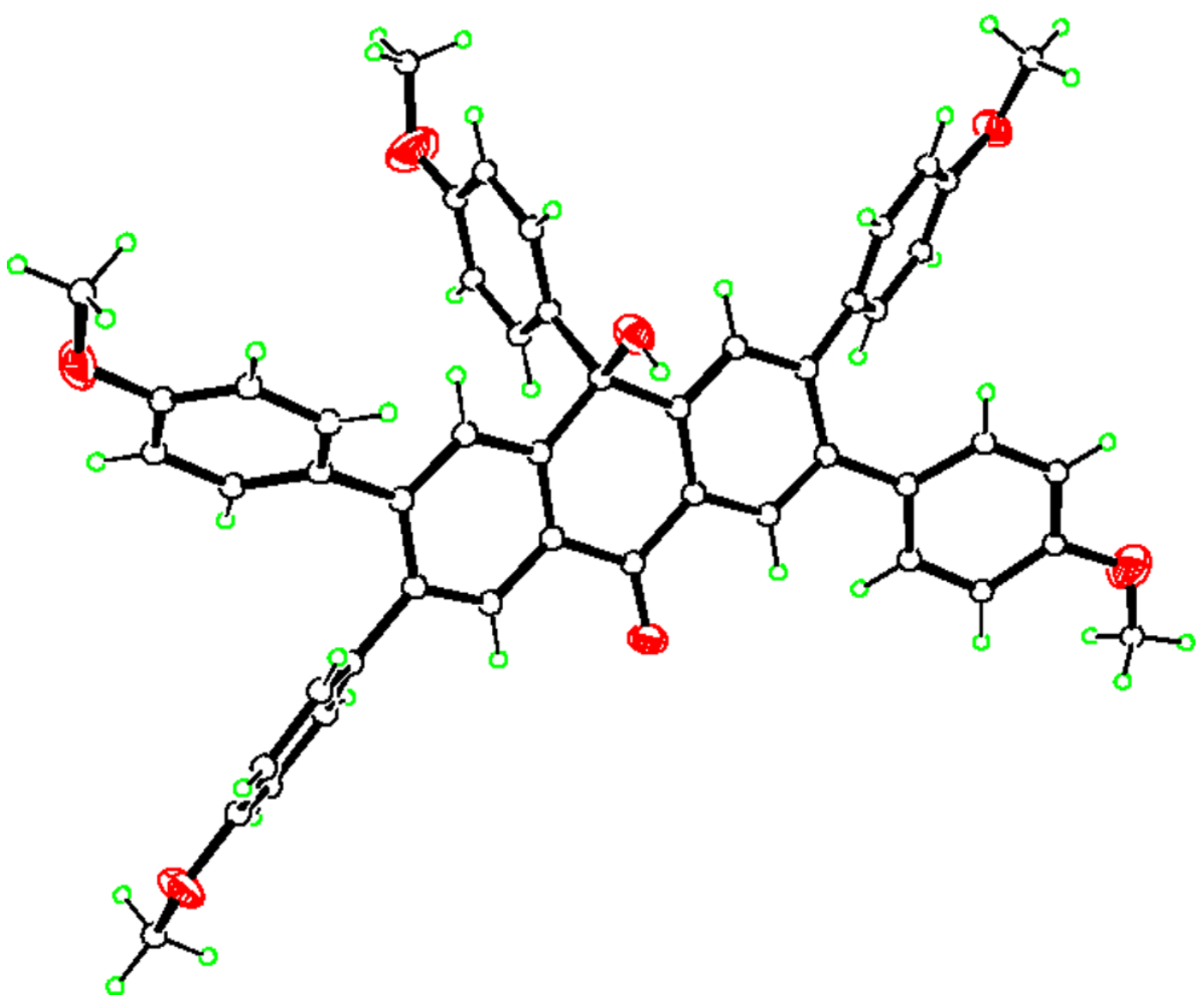

Figure S66 single crystal structures of $\mathbf{4 b}$, in ORTEP drawing with thermal ellipsoids at $30 \%$ probability. 


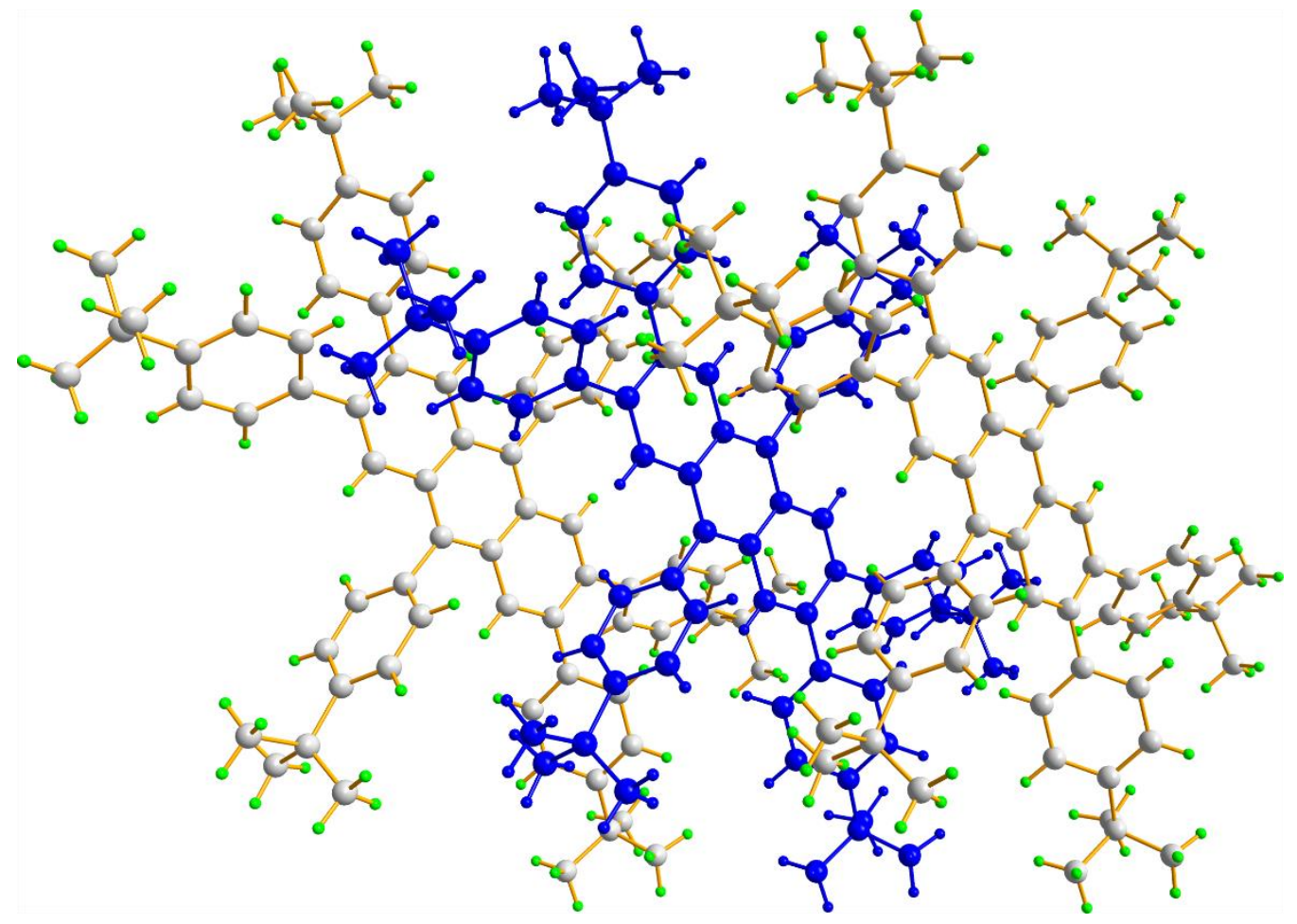

Figure S67 the crystal packing of 2,3,6,7,9,10-hexa-(4-tert-butyl-phenyl) anthracene 2a

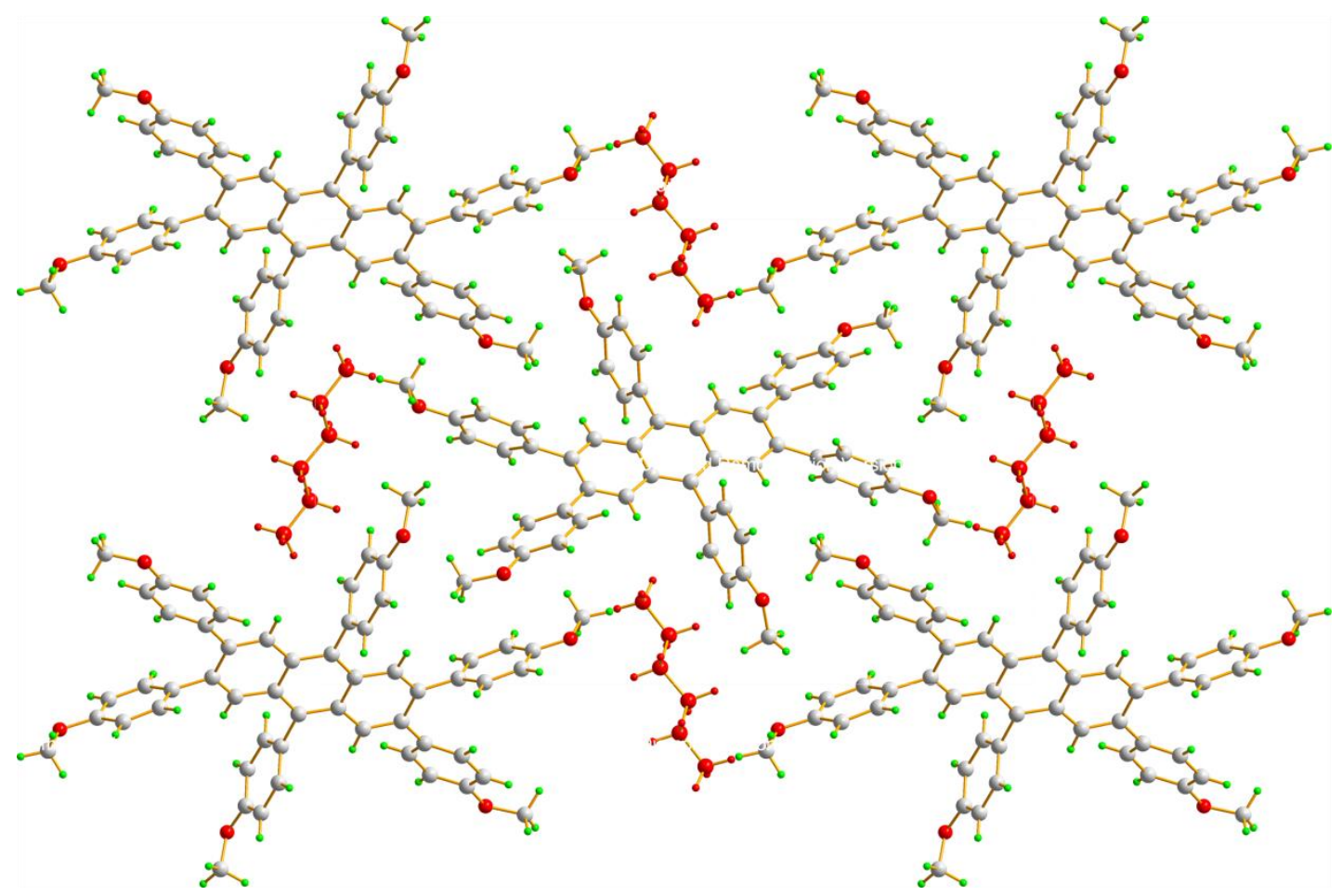

Figure S68 the packing structure of $\mathbf{2 b}$ 


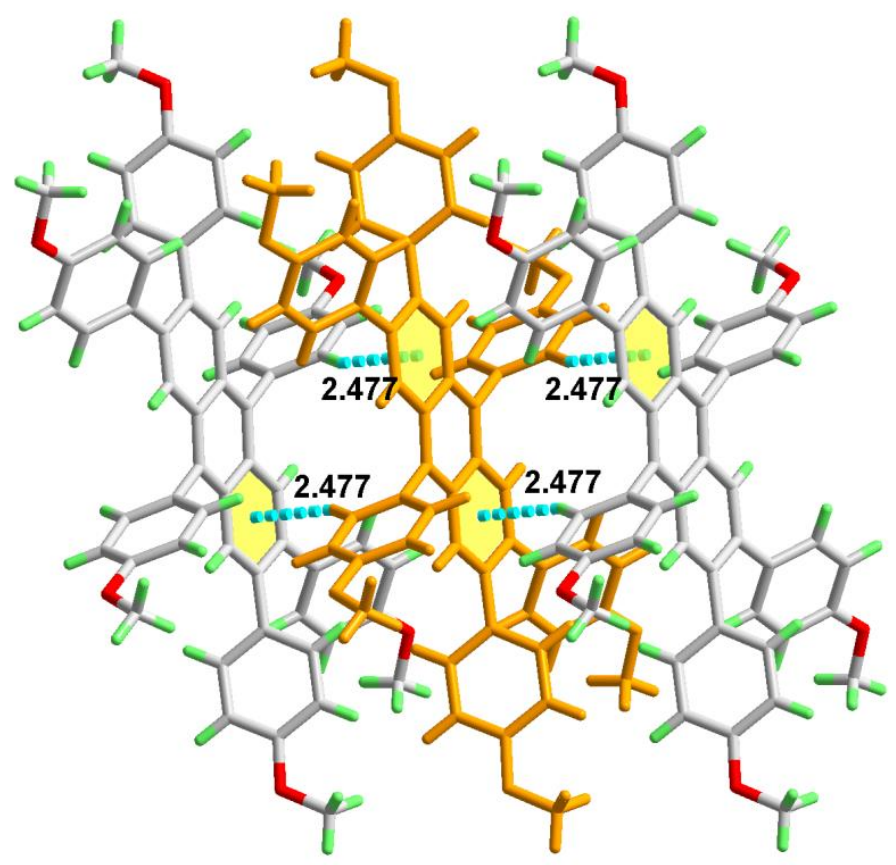

Figure $\mathbf{S 6 9}$ the packing structure of $\mathbf{2 b}$

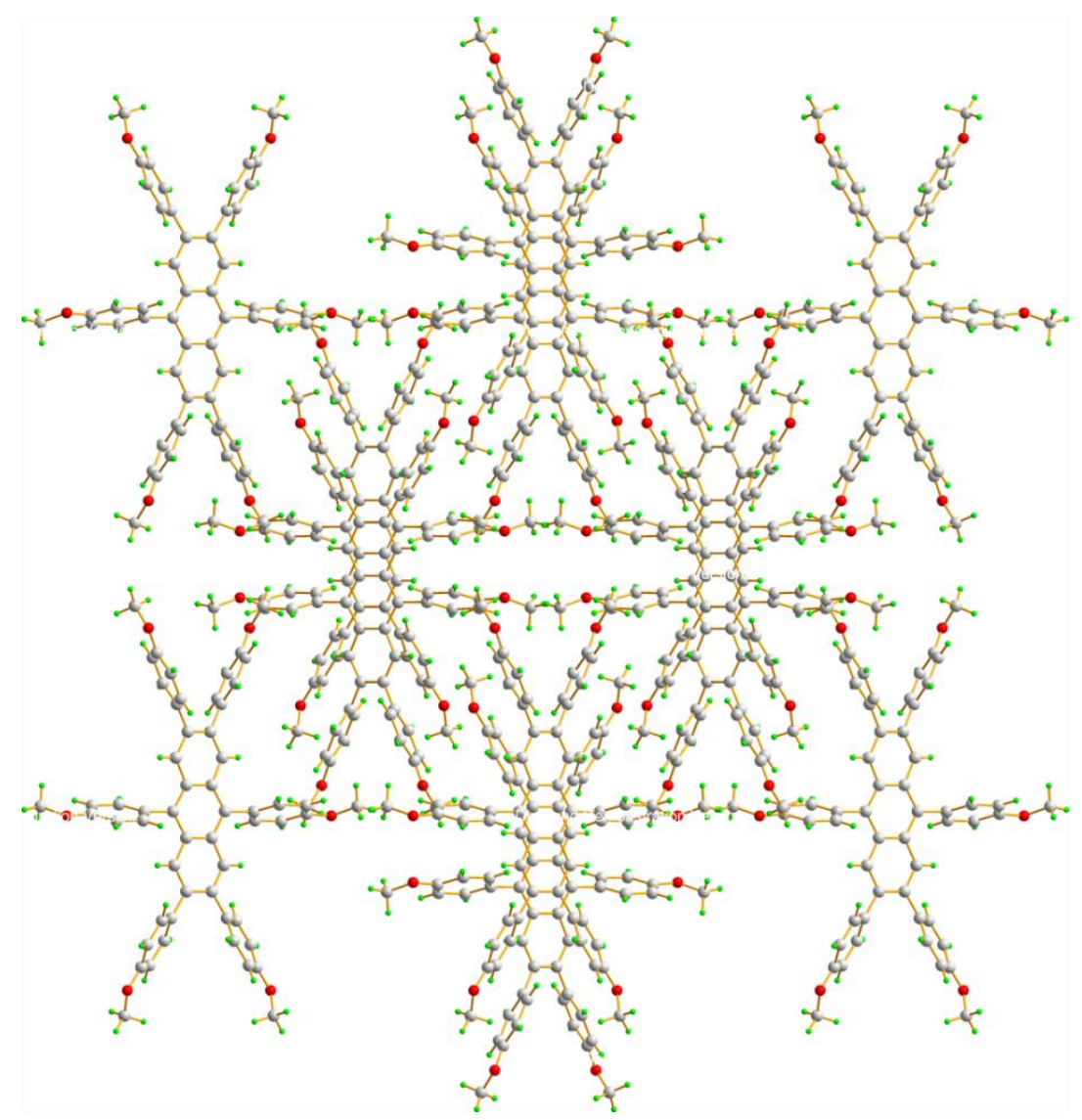

Figure S70 the packing structure of $\mathbf{2 b}$ 


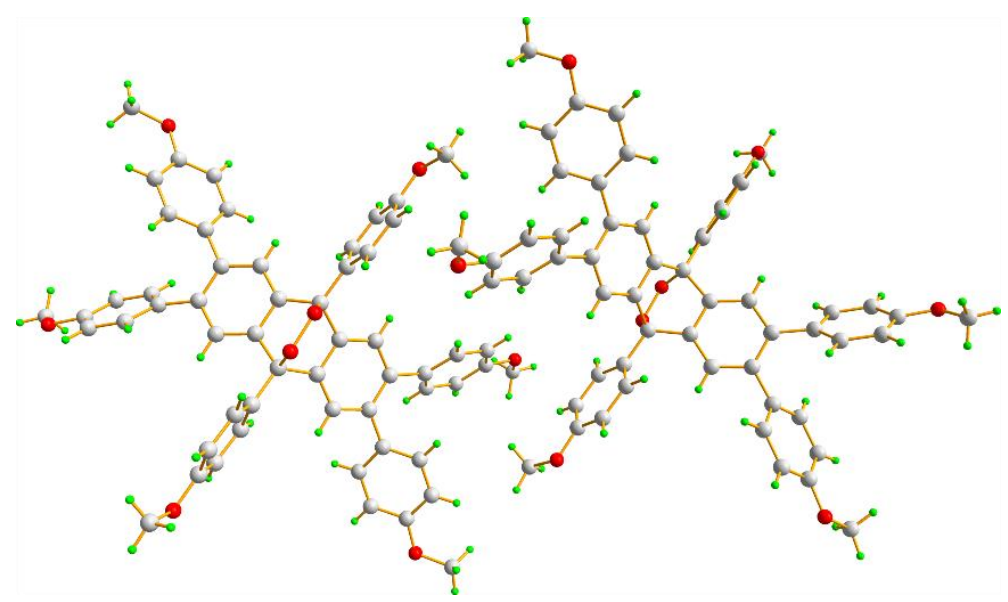

Figure S71 the packing structure of $\mathbf{3 b}$

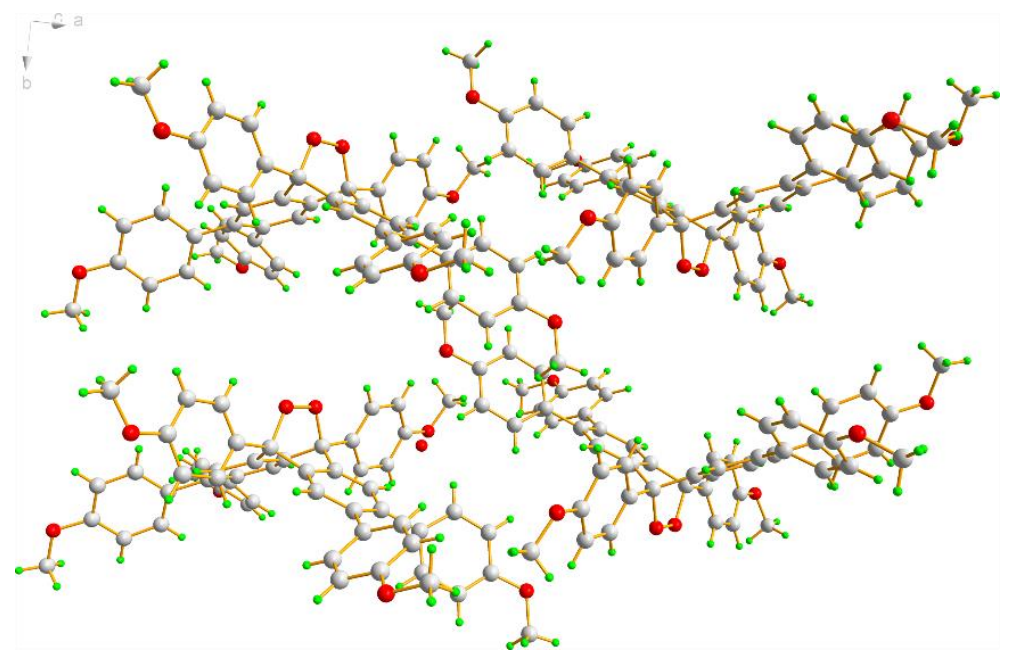

Figure S72 the packing structure of $\mathbf{3 b}$

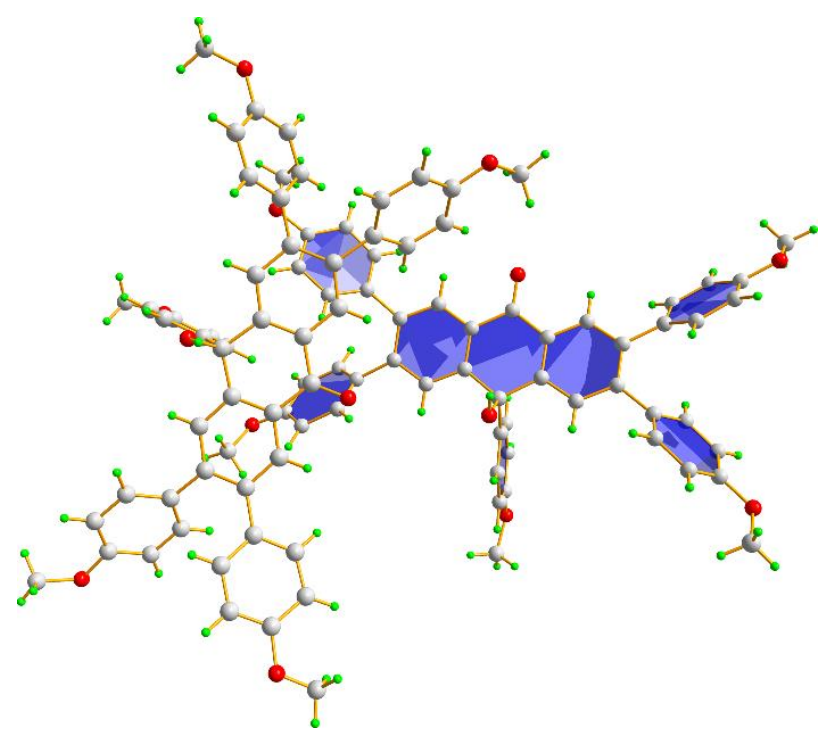

Figure S73 the packing structure of $\mathbf{4 b}$ 


\section{Photophysical Properties}

Table S3 The photophysical and electrochemical properties of compounds 2a-2d

\begin{tabular}{lllllll}
\hline $\mathrm{R}$ & $\begin{array}{l}\lambda_{\max \text { abs }}(\mathrm{nm}) \\
\left(\varepsilon \mathrm{M}^{-1} \mathrm{~cm}^{-1}\right)\end{array}$ & $\begin{array}{l}\lambda_{\operatorname{maxPL}} \\
(\mathrm{nm})\end{array}$ & $\Phi_{f}{ }^{a}$ & $\begin{array}{l}\mathrm{HOMO} \\
(\mathrm{eV})\end{array}$ & LUMO(eV) & $\begin{array}{l}\text { Energy gap } \\
(\mathrm{eV})\end{array}$ \\
\hline $\mathbf{2 a}(\mathbf{t B u})$ & $\begin{array}{l}317 \\
\left(2.03 \times 10^{5}\right)\end{array}$ & 445,467 & 0.59 & -5.12 & -1.88 & 3.24 \\
$\mathbf{2 b}(\mathbf{O M e})$ & $\begin{array}{l}322 \\
\left(1.81 \times 10^{5}\right)\end{array}$ & 448,470 & 0.46 & -4.95 & -1.74 & 3.21 \\
$\mathbf{2 c}(\mathbf{C H O})$ & $\begin{array}{l}334 \\
\left(5.95 \times 10^{4}\right)\end{array}$ & 458 & 0.48 & -6.12 & -2.94 & 3.18 \\
$\mathbf{2 d}\left(\mathbf{C F}_{3}\right)$ & $\begin{array}{l}313 \\
\left(1.18 \times 10^{5}\right)\end{array}$ & 438,461 & 0.52 & -6.15 & -2.88 & 3.27
\end{tabular}

${ }^{\text {a }}$ Measured in $\mathrm{CH}_{2} \mathrm{Cl}_{2}$ at room temperature DFT/B3LYP/6-31G* using Gaussian. ${ }^{\mathrm{c}}$ Calculated from the empirical formulae $\mathrm{HOMO}=-\left(\mathrm{E}_{\mathrm{ox}}+4.8\right),{ }_{\mathrm{L}}^{\mathrm{d}} \mathrm{LMO}=\mathrm{HOMO}+\mathrm{E}_{\mathrm{g}},{ }^{\mathrm{e}} \mathrm{C}$ Calculated from $\lambda_{\text {edge }}$.
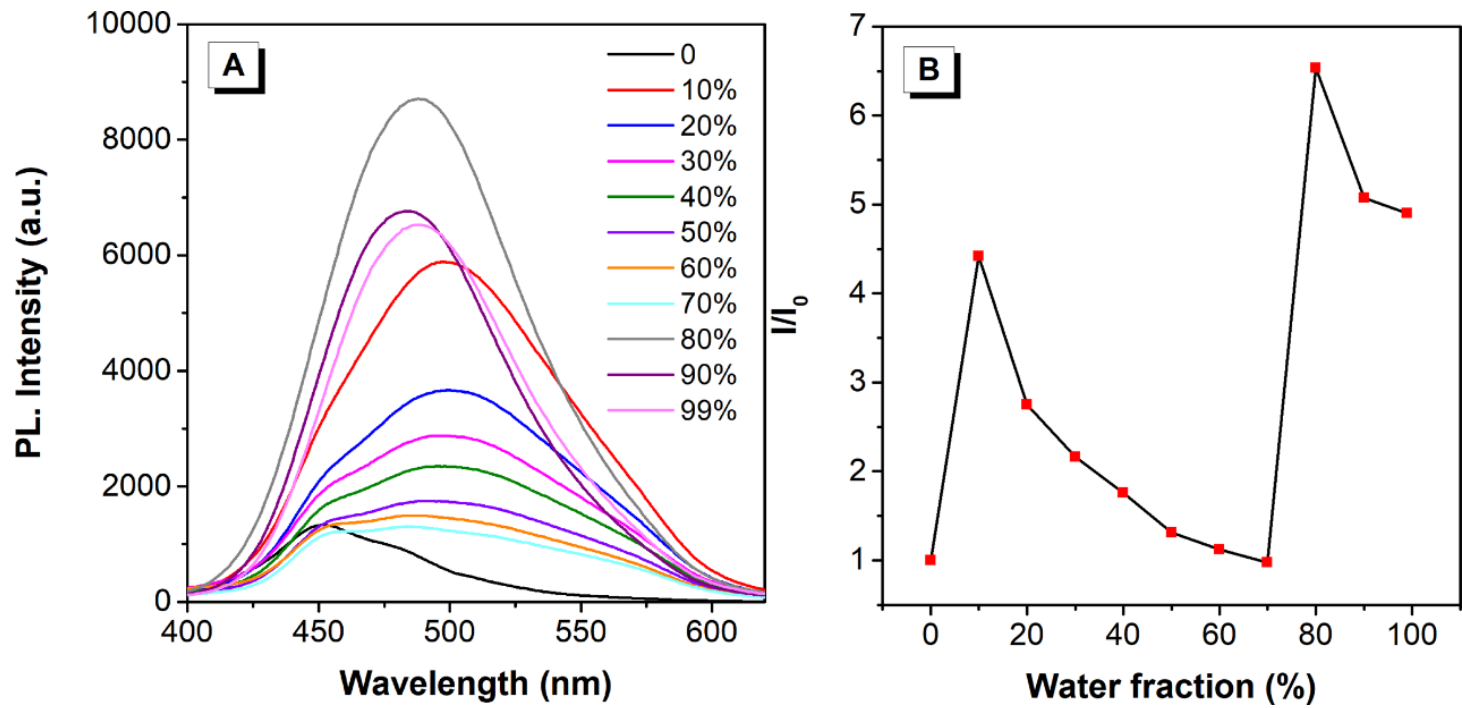

Figure S74 (A) PL spectra of $\mathbf{5 b}$ in THF/water mixtures with different water fractions $\left(f_{\mathrm{w}}\right)$; (B) plot of relative PL intensity $\left(\mathrm{I} / \mathrm{I}_{0}\right)$ and versus the composition of the THF/water mixture of $\mathbf{5 b}$. 


\section{Thermal Properties}

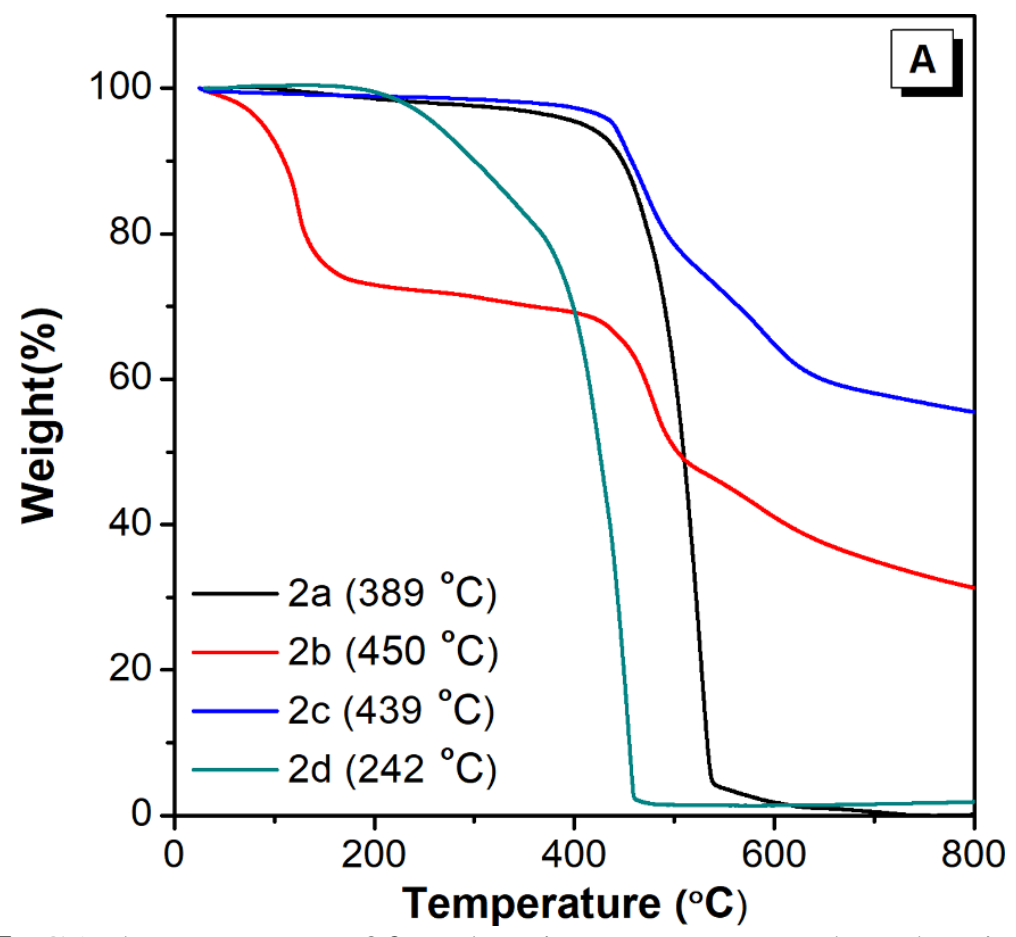

Figure S75TGA thermograms of $\mathbf{2}$ under nitrogen at atmosphere heating rate of 10 ${ }^{\circ} \mathrm{C} / \mathrm{min}$. 


\section{DFT calculation}

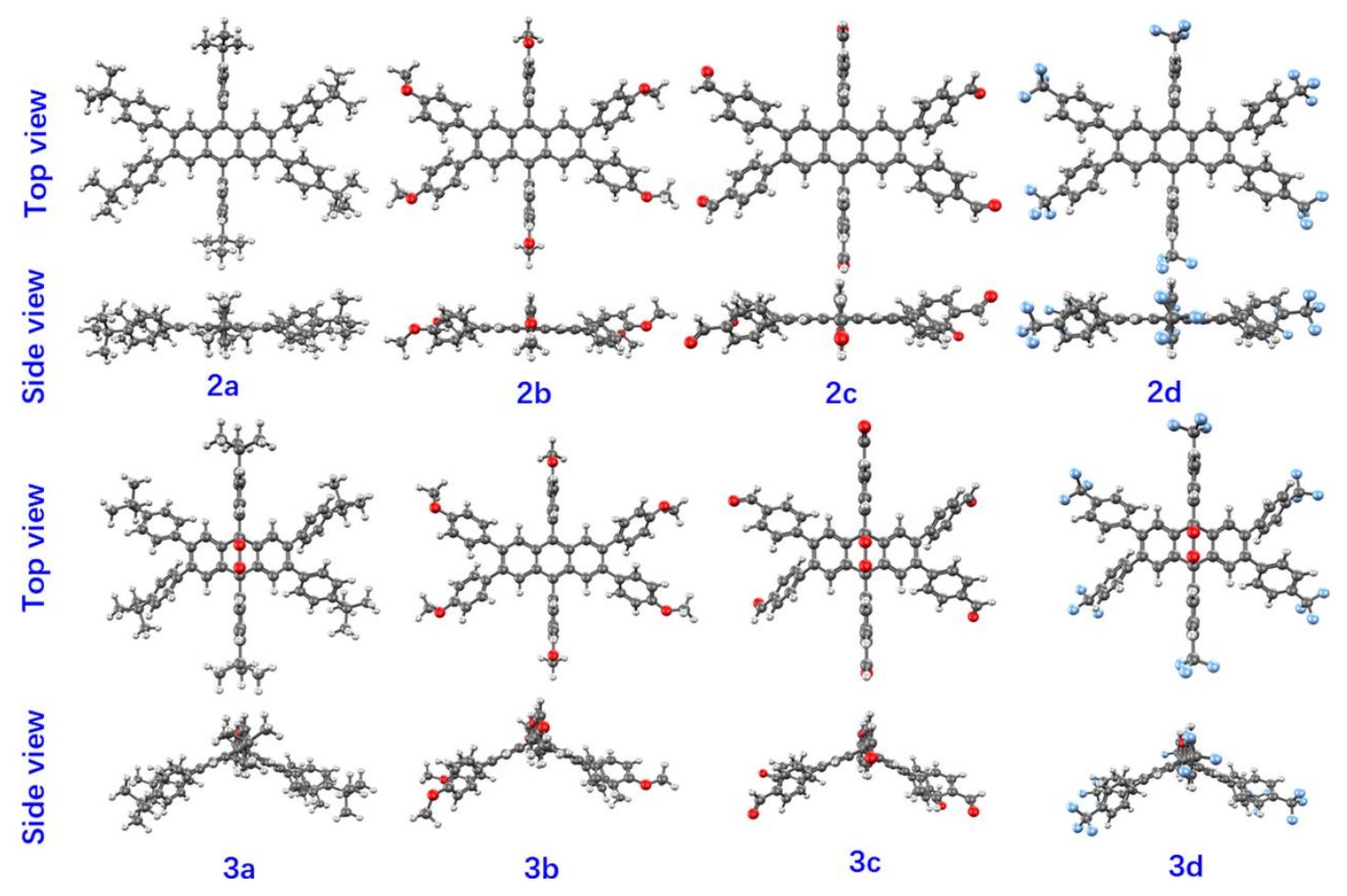

Figure S76 DFT calculation of the optimized molecular of 2 and 3 (B3LYP/6-31+G**) 
Table S4 Gibbs Free Energies (in $\mathrm{kcal} / \mathrm{mol}$ ) of reactions of Hexaaryl-substituted Anthracenes 2 and Corresponding EPOs, and their Energy Levels of HOMO and LUMO, calculated at B3LYP-D3/6-311G(d,p)/SMD(Chloroform) level, Gaussian 09.

\begin{tabular}{|c|c|c|c|c|c|}
\hline & \multirow{2}{*}{$\Delta G^{\mathrm{a}}$} & \multicolumn{2}{|c|}{2} & \multicolumn{2}{|c|}{3} \\
\hline & & HOMO & LUMO & HOMO & LUMO \\
\hline $2 a$ & -23.66 & -5.203 & -1.952 & -5.779 & -1.315 \\
\hline $2 b$ & -24.58 & -5.111 & -1.899 & -5.481 & -1.217 \\
\hline 2c & -21.43 & -5.722 & -2.560 & -6.435 & -2.317 \\
\hline $2 d$ & -19.60 & -5.714 & -2.454 & -6.468 & -1.923 \\
\hline
\end{tabular}

Table S5 The Absolute Energies of All Optimized Structures (in Hartree).

\begin{tabular}{cccccccc}
\hline Species & $\begin{array}{c}\text { Thermal } \\
\text { correction to } \\
\text { Energy }\end{array}$ & $\begin{array}{c}\text { Thermal } \\
\text { correction to } \\
\text { Enthalpy }\end{array}$ & $\begin{array}{c}\text { Thermal } \\
\text { correction to } \\
\text { Gibbs Free } \\
\text { Energy }\end{array}$ & $\begin{array}{c}\text { Single-point } \\
\text { energy }\end{array}$ & Energy & $\begin{array}{c}\text { Enthalpy } \\
\text { Gibbs Free } \\
\text { Energy }\end{array}$ \\
\hline $\mathbf{1} \mathbf{O}_{\mathbf{2}}$ & 0.006071 & 0.007015 & -0.015221 & -150.308500 & -150.302429 & -150.301485 & -150.323721 \\
$\mathbf{2 a}$ & 1.420238 & 1.421183 & 1.238250 & -2870.338490 & -2868.918252 & -2868.917307 & -2869.100240 \\
$\mathbf{2 b}$ & 0.924263 & 0.925207 & 0.776741 & -2613.825272 & -2612.901009 & -2612.900065 & -2613.048531 \\
$\mathbf{2 c}$ & 0.782292 & 0.783236 & 0.639955 & -2606.638554 & -2605.856262 & -2605.855318 & -2605.998599 \\
$\mathbf{2 d}$ & 0.762832 & 0.763776 & 0.590928 & -3949.383562 & -3948.620730 & -3948.619786 & -3948.792634 \\
$\mathbf{3 a}$ & 1.429689 & 1.430633 & 1.246629 & -3020.708290 & -3019.278601 & -3019.277657 & -3019.461661 \\
$\mathbf{3 b}$ & 0.933808 & 0.934752 & 0.784293 & -2764.195723 & -2763.261915 & -2763.260971 & -2763.411430 \\
$\mathbf{3 c}$ & 0.792361 & 0.793305 & 0.650013 & -2757.006477 & -2756.214116 & -2756.213172 & -2756.356464 \\
$\mathbf{3 d}$ & 0.773007 & 0.773951 & 0.604298 & -4099.751890 & -4098.978883 & -4098.977939 & -4099.147592 \\
\hline
\end{tabular}



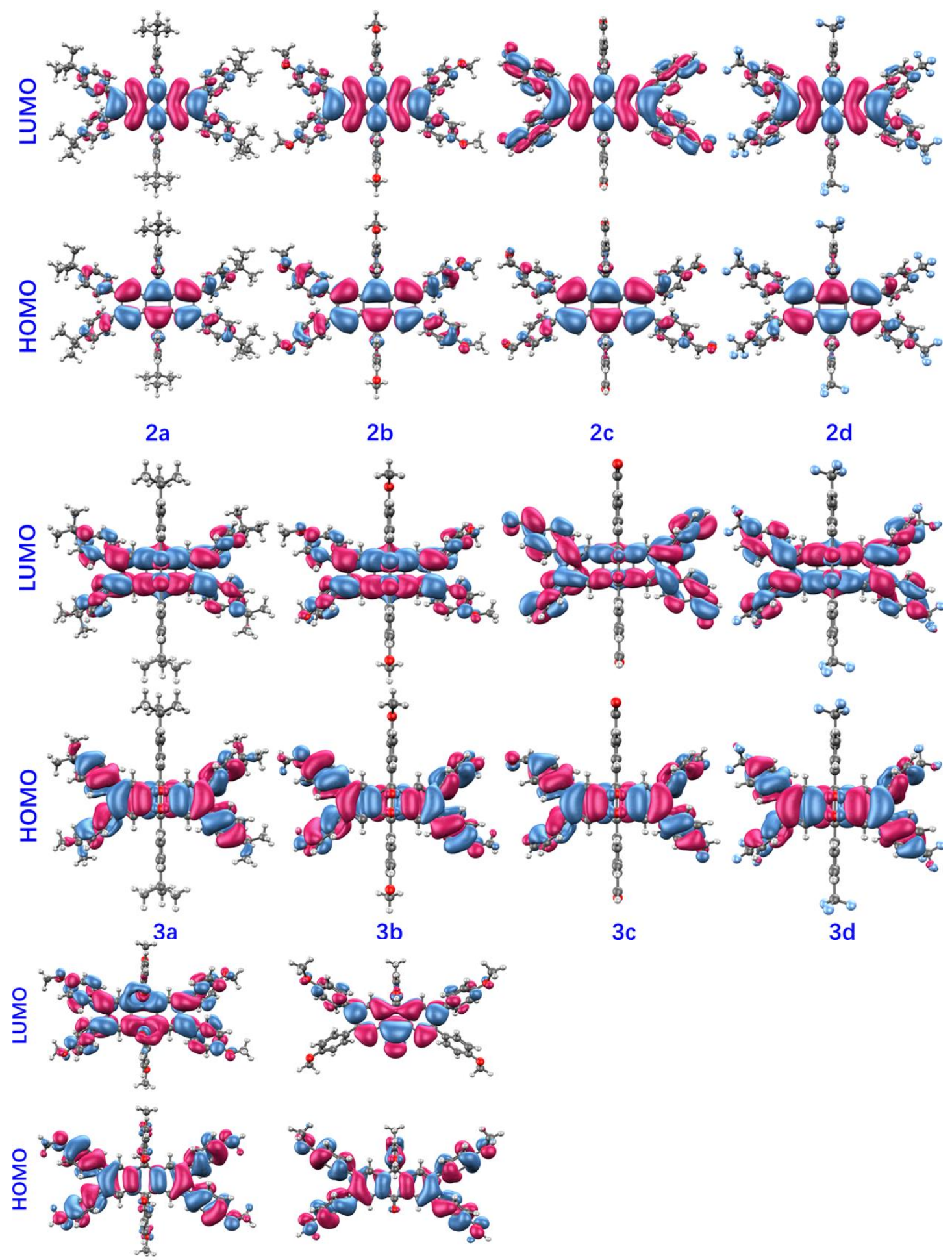

4b' 4b

Figure S77Frontier molecular orbitals of the highest occupied molecular orbital (HOMO) and the lowest unoccupied molecular orbital (LUMO) of compound 2-4. 


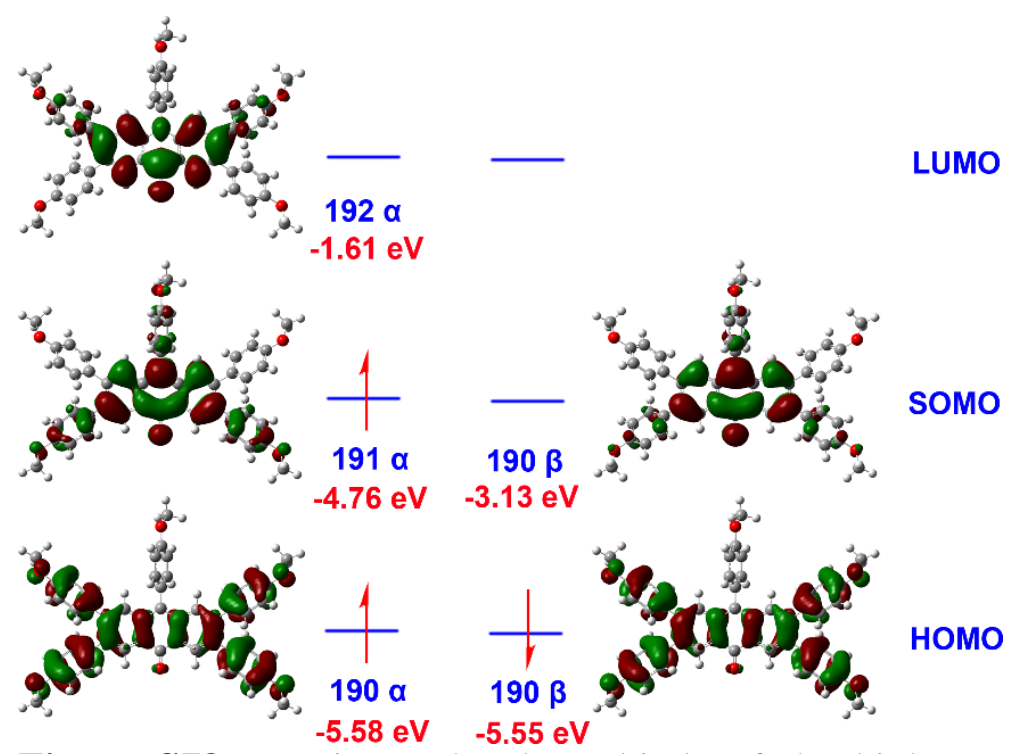

Figure S78 Frontier molecular orbitals of the highest occupied molecular orbital (HOMO) and the lowest unoccupied molecular orbital (LUMO) of compound $\mathbf{5 b}$. 
Table S6 Atom coordinates and absolute energies for $\mathbf{2 a}(t \mathbf{B u})$ Standard orientation

\begin{tabular}{|c|c|c|c|}
\hline Atomic Type & $\begin{array}{l}\text { Coordinates } \\
\text { (Angstroms) }\end{array}$ & Atomic Type & $\begin{array}{l}\text { Coordinates } \\
\text { (Angstroms) }\end{array}$ \\
\hline $\mathrm{C}$ & 3.677585 & 0.731677 & -0.020214 \\
\hline $\mathrm{C}$ & 3.681059 & -0.713026 & 0.042341 \\
\hline $\mathrm{C}$ & 2.480844 & -1.382062 & 0.045822 \\
\hline $\mathrm{C}$ & 1.219686 & -0.716312 & 0.017995 \\
\hline $\mathrm{C}$ & 1.216136 & 0.724012 & 0.012036 \\
\hline $\mathrm{C}$ & 2.474342 & 1.395475 & -0.019492 \\
\hline $\mathrm{C}$ & 0.003575 & -1.426528 & 0.015175 \\
\hline $\mathrm{C}$ & -1.216082 & -0.722478 & 0.014346 \\
\hline $\mathrm{C}$ & -1.219953 & 0.717917 & 0.023505 \\
\hline $\mathrm{C}$ & -0.003677 & 1.428114 & 0.018247 \\
\hline $\mathrm{C}$ & -2.473736 & -1.394845 & -0.014825 \\
\hline $\mathrm{C}$ & -3.677183 & -0.731904 & -0.008461 \\
\hline $\mathrm{C}$ & -3.681082 & 0.712617 & 0.059209 \\
\hline $\mathrm{C}$ & -2.481661 & 1.382966 & 0.057468 \\
\hline $\mathrm{C}$ & -0.007824 & 2.921225 & 0.017376 \\
\hline $\mathrm{C}$ & 0.007656 & -2.919632 & 0.010645 \\
\hline $\mathrm{C}$ & -4.941186 & 1.495231 & 0.170150 \\
\hline $\mathrm{C}$ & -4.931879 & -1.524406 & -0.110136 \\
\hline $\mathrm{C}$ & 4.932534 & 1.523539 & -0.123350 \\
\hline $\mathrm{C}$ & 4.939237 & -1.500383 & 0.141218 \\
\hline $\mathrm{C}$ & 5.166728 & -2.595401 & -0.696927 \\
\hline $\mathrm{C}$ & 6.337949 & -3.347660 & -0.602400 \\
\hline $\mathrm{C}$ & 7.327859 & -3.040948 & 0.336271 \\
\hline $\mathrm{C}$ & 7.085676 & -1.946929 & 1.183754 \\
\hline $\mathrm{C}$ & 5.925052 & -1.192220 & 1.090298 \\
\hline $\mathrm{C}$ & 5.160595 & 2.617837 & 0.715500 \\
\hline $\mathrm{C}$ & 6.330309 & 3.372165 & 0.618380 \\
\hline $\mathrm{C}$ & 7.317900 & 3.068343 & -0.323687 \\
\hline $\mathrm{C}$ & 7.074682 & 1.975484 & -1.172362 \\
\hline $\mathrm{C}$ & 5.915642 & 1.218738 & -1.076330 \\
\hline $\mathrm{C}$ & -0.041321 & -3.643272 & 1.202486 \\
\hline $\mathrm{C}$ & -0.035552 & -5.038788 & 1.195788 \\
\hline $\mathrm{C}$ & 0.016593 & -5.762888 & -0.000062 \\
\hline $\mathrm{C}$ & 0.065755 & -5.024154 & -1.192941 \\
\hline $\mathrm{C}$ & 0.062012 & -3.633939 & -1.191520 \\
\hline $\mathrm{C}$ & 0.040081 & 3.641872 & 1.211087 \\
\hline $\mathrm{C}$ & 0.033779 & 5.037392 & 1.207928 \\
\hline $\mathrm{C}$ & -0.017890 & 5.764507 & 0.013880 \\
\hline $\mathrm{C}$ & -0.065825 & 5.028774 & -1.180908 \\
\hline $\mathrm{C}$ & -0.061507 & 3.638550 & -1.183009 \\
\hline $\mathrm{C}$ & -5.913754 & 1.183027 & 1.125156 \\
\hline $\mathrm{C}$ & -7.087192 & 1.926035 & 1.228384 \\
\hline $\mathrm{C}$ & -7.346020 & 3.008611 & 0.379473 \\
\hline $\mathrm{C}$ & -6.360427 & 3.323561 & -0.568806 \\
\hline $\mathrm{C}$ & -5.184488 & 2.589401 & -0.671313 \\
\hline $\mathrm{C}$ & -5.156645 & -2.621654 & 0.725618 \\
\hline $\mathrm{C}$ & -6.325605 & -3.377259 & 0.628559 \\
\hline
\end{tabular}




\begin{tabular}{|c|c|c|c|}
\hline $\mathrm{C}$ & -7.315428 & -3.071900 & -0.310593 \\
\hline $\mathrm{C}$ & -7.075432 & -1.976001 & -1.156329 \\
\hline $\mathrm{C}$ & -5.917444 & -1.217755 & -1.059930 \\
\hline $\mathrm{C}$ & -8.644389 & 3.830034 & 0.439220 \\
\hline $\mathrm{C}$ & -9.593169 & 3.347547 & 1.549971 \\
\hline $\mathrm{C}$ & -9.382857 & 3.708407 & -0.913032 \\
\hline $\mathrm{C}$ & -8.305401 & 5.314045 & 0.703995 \\
\hline $\mathrm{C}$ & -8.626238 & -3.864358 & -0.439132 \\
\hline $\mathrm{C}$ & -8.704642 & -5.033512 & 0.557134 \\
\hline $\mathrm{C}$ & -8.741597 & -4.440367 & -1.868269 \\
\hline $\mathrm{C}$ & -9.819296 & -2.919398 & -0.170306 \\
\hline $\mathrm{C}$ & 0.034115 & -7.299855 & -0.048690 \\
\hline $\mathrm{C}$ & -0.079398 & -7.930360 & 1.349915 \\
\hline $\mathrm{C}$ & 1.360916 & -7.771204 & -0.686364 \\
\hline $\mathrm{C}$ & -1.149824 & -7.805859 & -0.903238 \\
\hline $\mathrm{C}$ & -0.036278 & 7.301592 & -0.030805 \\
\hline $\mathrm{C}$ & 0.075597 & 7.928541 & 1.369532 \\
\hline $\mathrm{C}$ & -1.362800 & 7.773762 & -0.668449 \\
\hline $\mathrm{C}$ & 1.148109 & 7.810589 & -0.882926 \\
\hline $\mathrm{C}$ & 8.629706 & 3.859186 & -0.452073 \\
\hline $\mathrm{C}$ & 8.711234 & 5.025769 & 0.546968 \\
\hline $\mathrm{C}$ & 8.744025 & 4.438508 & -1.879960 \\
\hline $\mathrm{C}$ & 9.821641 & 2.911718 & -0.187259 \\
\hline $\mathrm{C}$ & 8.640509 & -3.830683 & 0.462790 \\
\hline $\mathrm{C}$ & 8.720926 & -4.998094 & -0.535393 \\
\hline $\mathrm{C}$ & 8.757665 & -4.408788 & 1.890954 \\
\hline $\mathrm{C}$ & 9.831452 & -2.882814 & 0.195057 \\
\hline $\mathrm{H}$ & 2.486144 & -2.462447 & 0.110974 \\
\hline $\mathrm{H}$ & 2.474742 & 2.475968 & -0.083820 \\
\hline $\mathrm{H}$ & -2.473519 & -2.475088 & -0.082879 \\
\hline $\mathrm{H}$ & -2.487686 & 2.463324 & 0.124328 \\
\hline $\mathrm{H}$ & 4.427226 & -2.853855 & -1.447166 \\
\hline $\mathrm{H}$ & 6.466569 & -4.178152 & -1.283917 \\
\hline $\mathrm{H}$ & 7.820056 & -1.673825 & 1.932565 \\
\hline $\mathrm{H}$ & 5.779626 & -0.352918 & 1.759883 \\
\hline $\mathrm{H}$ & 4.423166 & 2.873601 & 1.468704 \\
\hline $\mathrm{H}$ & 6.459850 & 4.201758 & 1.300825 \\
\hline $\mathrm{H}$ & 7.807204 & 1.704643 & -1.923809 \\
\hline $\mathrm{H}$ & 5.769486 & 0.380038 & -1.746518 \\
\hline $\mathrm{H}$ & -0.081648 & -3.112475 & 2.147722 \\
\hline $\mathrm{H}$ & -0.073258 & -5.553739 & 2.146626 \\
\hline $\mathrm{H}$ & 0.109428 & -5.539718 & -2.145362 \\
\hline $\mathrm{H}$ & 0.099269 & -3.093941 & -2.131623 \\
\hline $\mathrm{H}$ & 0.079853 & 3.108677 & 2.155000 \\
\hline $\mathrm{H}$ & 0.070596 & 5.549928 & 2.160106 \\
\hline $\mathrm{H}$ & -0.109085 & 5.546708 & -2.132067 \\
\hline $\mathrm{H}$ & -0.097909 & 3.100919 & -2.124499 \\
\hline $\mathrm{H}$ & -5.755821 & 0.346889 & 1.795966 \\
\hline $\mathrm{H}$ & -7.804808 & 1.639884 & 1.985638 \\
\hline $\mathrm{H}$ & -6.512555 & 4.153158 & -1.249777 \\
\hline
\end{tabular}




\begin{tabular}{|c|c|c|c|}
\hline $\mathrm{H}$ & -4.452173 & 2.852845 & -1.426858 \\
\hline $\mathrm{H}$ & -4.417200 & -2.878966 & 1.476319 \\
\hline $\mathrm{H}$ & -6.452617 & -4.209176 & 1.308646 \\
\hline $\mathrm{H}$ & -7.809625 & -1.704139 & -1.905800 \\
\hline $\mathrm{H}$ & -5.773707 & -0.376691 & -1.727711 \\
\hline $\mathrm{H}$ & -9.134715 & 3.427551 & 2.539720 \\
\hline $\mathrm{H}$ & -10.496935 & 3.963547 & 1.554419 \\
\hline $\mathrm{H}$ & -9.902713 & 2.309766 & 1.397925 \\
\hline $\mathrm{H}$ & -8.780293 & 4.092373 & -1.739863 \\
\hline $\mathrm{H}$ & -10.317848 & 4.277616 & -0.889083 \\
\hline $\mathrm{H}$ & -9.625946 & 2.664195 & -1.130952 \\
\hline $\mathrm{H}$ & -9.222346 & 5.910113 & 0.750235 \\
\hline $\mathrm{H}$ & -7.776941 & 5.430156 & 1.654722 \\
\hline $\mathrm{H}$ & -7.675022 & 5.733778 & -0.083632 \\
\hline $\mathrm{H}$ & -8.661654 & -4.687890 & 1.593934 \\
\hline $\mathrm{H}$ & -9.651585 & -5.564495 & 0.424963 \\
\hline $\mathrm{H}$ & -7.897077 & -5.754541 & 0.401561 \\
\hline $\mathrm{H}$ & -8.744352 & -3.652787 & -2.625563 \\
\hline $\mathrm{H}$ & -9.671694 & -5.008048 & -1.973593 \\
\hline $\mathrm{H}$ & -7.906788 & -5.113104 & -2.085721 \\
\hline $\mathrm{H}$ & -10.763855 & -3.466600 & -0.253815 \\
\hline $\mathrm{H}$ & -9.760178 & -2.494880 & 0.836240 \\
\hline $\mathrm{H}$ & -9.849921 & -2.090812 & -0.882184 \\
\hline $\mathrm{H}$ & -1.007798 & -7.641875 & 1.851153 \\
\hline $\mathrm{H}$ & -0.075405 & -9.020386 & 1.261903 \\
\hline $\mathrm{H}$ & 0.759606 & -7.649473 & 1.992745 \\
\hline $\mathrm{H}$ & 1.476245 & -7.393853 & -1.705457 \\
\hline $\mathrm{H}$ & 1.394603 & -8.864603 & -0.728416 \\
\hline $\mathrm{H}$ & 2.218729 & -7.426309 & -0.101408 \\
\hline $\mathrm{H}$ & -1.148971 & -8.899890 & -0.939031 \\
\hline $\mathrm{H}$ & -2.105154 & -7.480918 & -0.480849 \\
\hline $\mathrm{H}$ & -1.096451 & -7.440662 & -1.931727 \\
\hline $\mathrm{H}$ & 1.003691 & 7.639253 & 1.870876 \\
\hline $\mathrm{H}$ & 0.071112 & 9.018792 & 1.284357 \\
\hline $\mathrm{H}$ & -0.763845 & 7.645551 & 2.010859 \\
\hline $\mathrm{H}$ & -1.476975 & 7.398888 & -1.688590 \\
\hline $\mathrm{H}$ & -1.397088 & 8.867244 & -0.707787 \\
\hline $\mathrm{H}$ & -2.220920 & 7.426887 & -0.085124 \\
\hline $\mathrm{H}$ & 1.146652 & 8.904718 & -0.915766 \\
\hline $\mathrm{H}$ & 2.103261 & 7.485060 & -0.460582 \\
\hline $\mathrm{H}$ & 1.095871 & 7.448192 & -1.912459 \\
\hline $\mathrm{H}$ & 8.669327 & 4.677624 & 1.582972 \\
\hline $\mathrm{H}$ & 9.658744 & 5.555736 & 0.414753 \\
\hline $\mathrm{H}$ & 7.904472 & 5.748372 & 0.394483 \\
\hline $\mathrm{H}$ & 8.745305 & 3.652683 & -2.639075 \\
\hline $\mathrm{H}$ & 9.674560 & 5.005527 & -1.984948 \\
\hline $\mathrm{H}$ & 7.909641 & 5.112550 & -2.094988 \\
\hline $\mathrm{H}$ & 10.766934 & 3.457628 & -0.270924 \\
\hline $\mathrm{H}$ & 9.763361 & 2.484935 & 0.818369 \\
\hline $\mathrm{H}$ & 9.849777 & 2.084731 & -0.901042 \\
\hline
\end{tabular}




\begin{tabular}{llll}
\hline $\mathrm{H}$ & 8.676684 & -4.650903 & -1.571619 \\
$\mathrm{H}$ & 9.669089 & -5.527236 & -0.404562 \\
$\mathrm{H}$ & 7.915032 & -5.721180 & -0.380648 \\
$\mathrm{H}$ & 8.759352 & -3.622370 & 2.649455 \\
$\mathrm{H}$ & 9.688878 & -4.974888 & 1.994910 \\
$\mathrm{H}$ & 7.924241 & -5.083454 & 2.107784 \\
$\mathrm{H}$ & 10.777143 & -3.428251 & 0.277260 \\
$\mathrm{H}$ & 9.771031 & -2.456732 & -0.810745 \\
$\mathrm{H}$ & 9.860657 & -2.055336 & 0.908228 \\
\hline
\end{tabular}


Table S7 Atom coordinates and absolute energies for 2b (OMe) Standard orientation

\begin{tabular}{|c|c|c|c|}
\hline Atomic Type & $\begin{array}{l}\text { Coordinates } \\
\text { (Angstroms) }\end{array}$ & Atomic Type & $\begin{array}{l}\text { Coordinates } \\
\text { (Angstroms) }\end{array}$ \\
\hline $\mathrm{C}$ & 3.659668 & 0.732505 & 0.014278 \\
\hline $\mathrm{C}$ & 3.674163 & -0.713552 & 0.041897 \\
\hline $\mathrm{C}$ & 2.477624 & -1.390517 & 0.042484 \\
\hline $\mathrm{C}$ & 1.210882 & -0.734996 & 0.037853 \\
\hline $\mathrm{C}$ & 1.196635 & 0.705424 & 0.052503 \\
\hline $\mathrm{C}$ & 2.450094 & 1.385606 & 0.031283 \\
\hline $\mathrm{C}$ & -0.000101 & -1.454715 & 0.041560 \\
\hline $\mathrm{C}$ & -1.224554 & -0.758244 & 0.056674 \\
\hline $\mathrm{C}$ & -1.239064 & 0.682049 & 0.074009 \\
\hline $\mathrm{C}$ & -0.028038 & 1.401799 & 0.070098 \\
\hline $\mathrm{C}$ & -2.478134 & -1.438219 & 0.039809 \\
\hline $\mathrm{C}$ & -3.687678 & -0.785678 & 0.060031 \\
\hline $\mathrm{C}$ & -3.702350 & 0.659457 & 0.127478 \\
\hline $\mathrm{C}$ & -2.505589 & 1.336585 & 0.119479 \\
\hline $\mathrm{C}$ & -0.040834 & 2.895055 & 0.090194 \\
\hline $\mathrm{C}$ & 0.012220 & -2.948009 & 0.043226 \\
\hline $\mathrm{C}$ & -4.963852 & 1.438096 & 0.247797 \\
\hline $\mathrm{C}$ & -4.934104 & -1.593913 & -0.016755 \\
\hline $\mathrm{C}$ & 4.905300 & 1.541848 & -0.065832 \\
\hline $\mathrm{C}$ & 4.937252 & -1.497179 & 0.102028 \\
\hline $\mathrm{C}$ & 5.155555 & -2.568401 & -0.779667 \\
\hline $\mathrm{C}$ & 6.316361 & -3.325099 & -0.721458 \\
\hline $\mathrm{C}$ & 7.299444 & -3.035446 & 0.232753 \\
\hline $\mathrm{C}$ & 7.097168 & -1.978249 & 1.126420 \\
\hline $\mathrm{C}$ & 5.928846 & -1.222861 & 1.049501 \\
\hline $\mathrm{C}$ & 5.112317 & 2.621664 & 0.808204 \\
\hline $\mathrm{C}$ & 6.254852 & 3.403775 & 0.730079 \\
\hline $\mathrm{C}$ & 7.230500 & 3.131932 & -0.237010 \\
\hline $\mathrm{C}$ & 7.039487 & 2.066078 & -1.122914 \\
\hline $\mathrm{C}$ & 5.889626 & 1.285001 & -1.025867 \\
\hline $\mathrm{C}$ & -0.009028 & -3.661180 & 1.250623 \\
\hline $\mathrm{C}$ & -0.001970 & -5.047939 & 1.262520 \\
\hline $\mathrm{C}$ & 0.023929 & -5.764048 & 0.058433 \\
\hline $\mathrm{C}$ & 0.046228 & -5.068925 & -1.154439 \\
\hline $\mathrm{C}$ & 0.041176 & -3.672520 & -1.148528 \\
\hline $\mathrm{C}$ & -0.024721 & 3.597093 & 1.304086 \\
\hline $\mathrm{C}$ & -0.032525 & 4.983852 & 1.328475 \\
\hline $\mathrm{C}$ & -0.054165 & 5.710875 & 0.130884 \\
\hline $\mathrm{C}$ & -0.071336 & 5.026852 & -1.088402 \\
\hline $\mathrm{C}$ & -0.065476 & 3.630558 & -1.095017 \\
\hline $\mathrm{C}$ & -5.941317 & 1.109163 & 1.203542 \\
\hline $\mathrm{C}$ & -7.095847 & 1.861137 & 1.337782 \\
\hline $\mathrm{C}$ & -7.318273 & 2.972273 & 0.512225 \\
\hline $\mathrm{C}$ & -6.359461 & 3.317744 & -0.443741 \\
\hline $\mathrm{C}$ & -5.198648 & 2.551923 & -0.563344 \\
\hline $\mathrm{C}$ & -5.126485 & -2.691197 & 0.838724 \\
\hline $\mathrm{C}$ & -6.272185 & -3.468938 & 0.766648 \\
\hline
\end{tabular}




\begin{tabular}{|c|c|c|c|}
\hline $\mathrm{C}$ & -7.265505 & -3.175193 & -0.175746 \\
\hline $\mathrm{C}$ & -7.088374 & -2.092838 & -1.044224 \\
\hline $\mathrm{C}$ & -5.934987 & -1.316101 & -0.953104 \\
\hline $\mathrm{O}$ & -8.487704 & 3.639510 & 0.718257 \\
\hline $\mathrm{C}$ & -8.755363 & 4.801910 & -0.065431 \\
\hline $\mathrm{O}$ & -8.354985 & -3.992298 & -0.164400 \\
\hline $\mathrm{C}$ & -9.386987 & -3.764978 & -1.123575 \\
\hline $\mathrm{O}$ & 0.025039 & -7.120720 & 0.177521 \\
\hline $\mathrm{C}$ & 0.034651 & -7.909837 & -1.011826 \\
\hline $\mathrm{O}$ & -0.056739 & 7.066640 & 0.261792 \\
\hline $\mathrm{C}$ & -0.062624 & 7.865293 & -0.921114 \\
\hline $\mathrm{O}$ & 8.317787 & 3.952330 & -0.231718 \\
\hline $\mathrm{C}$ & 9.334013 & 3.741666 & -1.211368 \\
\hline $\mathrm{O}$ & 8.405232 & -3.830255 & 0.207641 \\
\hline $\mathrm{C}$ & 9.423075 & -3.610414 & 1.183763 \\
\hline $\mathrm{H}$ & 2.490820 & -2.472070 & 0.084886 \\
\hline $\mathrm{H}$ & 2.441417 & 2.467187 & -0.010280 \\
\hline $\mathrm{H}$ & -2.470060 & -2.518436 & -0.028594 \\
\hline $\mathrm{H}$ & -2.517176 & 2.416387 & 0.193337 \\
\hline $\mathrm{H}$ & 4.407933 & -2.800244 & -1.530057 \\
\hline $\mathrm{H}$ & 6.486582 & -4.145299 & -1.409203 \\
\hline $\mathrm{H}$ & 7.834939 & -1.734831 & 1.879019 \\
\hline $\mathrm{H}$ & 5.789895 & -0.405473 & 1.746709 \\
\hline $\mathrm{H}$ & 4.371097 & 2.839831 & 1.568927 \\
\hline $\mathrm{H}$ & 6.416538 & 4.230269 & 1.412315 \\
\hline $\mathrm{H}$ & 7.771513 & 1.835790 & -1.885260 \\
\hline $\mathrm{H}$ & 5.759220 & 0.461112 & -1.717048 \\
\hline $\mathrm{H}$ & -0.029975 & -3.119191 & 2.189758 \\
\hline $\mathrm{H}$ & -0.017521 & -5.601515 & 2.194211 \\
\hline $\mathrm{H}$ & 0.066786 & -5.594285 & -2.099863 \\
\hline $\mathrm{H}$ & 0.056452 & -3.141647 & -2.094254 \\
\hline $\mathrm{H}$ & -0.007485 & 3.046652 & 2.238353 \\
\hline $\mathrm{H}$ & -0.020759 & 5.528870 & 2.265261 \\
\hline $\mathrm{H}$ & -0.087752 & 5.560732 & -2.029195 \\
\hline $\mathrm{H}$ & -0.076441 & 3.108119 & -2.045473 \\
\hline $\mathrm{H}$ & -5.789032 & 0.255680 & 1.852688 \\
\hline $\mathrm{H}$ & -7.844438 & 1.609283 & 2.079877 \\
\hline $\mathrm{H}$ & -6.503025 & 4.167212 & -1.097530 \\
\hline $\mathrm{H}$ & -4.467549 & 2.823712 & -1.316788 \\
\hline $\mathrm{H}$ & -4.371672 & -2.925897 & 1.580921 \\
\hline $\mathrm{H}$ & -6.423438 & -4.308373 & 1.435302 \\
\hline $\mathrm{H}$ & -7.834657 & -1.845586 & -1.787173 \\
\hline $\mathrm{H}$ & -5.815446 & -0.478220 & -1.629431 \\
\hline $\mathrm{H}$ & -9.724809 & 5.169624 & 0.269495 \\
\hline $\mathrm{H}$ & -7.999835 & 5.578396 & 0.098311 \\
\hline $\mathrm{H}$ & -8.811460 & 4.563774 & -1.133388 \\
\hline $\mathrm{H}$ & -10.140807 & -4.528596 & -0.934698 \\
\hline $\mathrm{H}$ & -9.840457 & -2.775139 & -1.000975 \\
\hline $\mathrm{H}$ & -9.013487 & -3.873601 & -2.147845 \\
\hline $\mathrm{H}$ & 0.026452 & -8.947416 & -0.679845 \\
\hline
\end{tabular}




\begin{tabular}{cccc}
\hline $\mathrm{H}$ & -0.852863 & -7.720936 & -1.625698 \\
$\mathrm{H}$ & 0.937086 & -7.729602 & -1.606251 \\
$\mathrm{H}$ & -0.056401 & 8.900210 & -0.580906 \\
$\mathrm{H}$ & 0.827183 & 7.682042 & -1.533302 \\
$\mathrm{H}$ & -0.962753 & 7.689050 & -1.520248 \\
$\mathrm{H}$ & 10.089943 & 4.503387 & -1.023254 \\
$\mathrm{H}$ & 9.790599 & 2.750653 & -1.111756 \\
$\mathrm{H}$ & 8.943589 & 3.865605 & -2.227531 \\
$\mathrm{H}$ & 10.192714 & -4.354941 & 0.982971 \\
$\mathrm{H}$ & 9.859656 & -2.609634 & 1.092187 \\
$\mathrm{H}$ & 9.040895 & -3.752457 & 2.200661 \\
\hline
\end{tabular}


Table S8 Atom coordinates and absolute energies for 2c (CHO) Standard orientation

\begin{tabular}{|c|c|c|c|}
\hline \multirow{2}{*}{ Atomic Type } & \multicolumn{3}{|c|}{ Coordinates (Angstroms) } \\
\hline & $\mathbf{X}$ & $\mathbf{Y}$ & $\mathbf{Z}$ \\
\hline $\mathrm{C}$ & 3.660302 & 0.744418 & 0.025811 \\
\hline $\mathrm{C}$ & 3.675891 & -0.699419 & 0.071413 \\
\hline $\mathrm{C}$ & 2.483212 & -1.380702 & 0.082389 \\
\hline $\mathrm{C}$ & 1.217967 & -0.723192 & 0.079146 \\
\hline $\mathrm{C}$ & 1.202790 & 0.716686 & 0.088153 \\
\hline $\mathrm{C}$ & 2.453651 & 1.400637 & 0.051864 \\
\hline $\mathrm{C}$ & 0.007238 & -1.440965 & 0.080548 \\
\hline $\mathrm{C}$ & -1.218274 & -0.749253 & 0.095584 \\
\hline $\mathrm{C}$ & -1.233805 & 0.690398 & 0.117351 \\
\hline $\mathrm{C}$ & -0.022940 & 1.408410 & 0.109393 \\
\hline $\mathrm{C}$ & -2.469137 & -1.433155 & 0.068250 \\
\hline $\mathrm{C}$ & -3.676235 & -0.777866 & 0.078832 \\
\hline $\mathrm{C}$ & -3.692210 & 0.665136 & 0.154911 \\
\hline $\mathrm{C}$ & -2.499223 & 1.346624 & 0.156441 \\
\hline $\mathrm{C}$ & -0.040139 & 2.901530 & 0.110219 \\
\hline $\mathrm{C}$ & 0.024283 & -2.933881 & 0.055507 \\
\hline $\mathrm{C}$ & -4.955325 & 1.438152 & 0.282744 \\
\hline $\mathrm{C}$ & -4.920517 & -1.581117 & -0.050551 \\
\hline $\mathrm{C}$ & 4.903899 & 1.546882 & -0.113344 \\
\hline $\mathrm{C}$ & 4.939745 & -1.476392 & 0.162713 \\
\hline $\mathrm{C}$ & 5.162682 & -2.567201 & -0.689241 \\
\hline $\mathrm{C}$ & 6.328913 & -3.316241 & -0.586030 \\
\hline $\mathrm{C}$ & 7.290885 & -2.993966 & 0.376566 \\
\hline $\mathrm{C}$ & 7.071076 & -1.907471 & 1.235385 \\
\hline $\mathrm{C}$ & 5.913837 & -1.156169 & 1.125795 \\
\hline $\mathrm{C}$ & 5.136096 & 2.645487 & 0.726042 \\
\hline $\mathrm{C}$ & 6.281412 & 3.418513 & 0.575976 \\
\hline $\mathrm{C}$ & 7.213376 & 3.112068 & -0.420907 \\
\hline $\mathrm{C}$ & 6.983834 & 2.018028 & -1.267431 \\
\hline $\mathrm{C}$ & 5.847377 & 1.243647 & -1.111917 \\
\hline $\mathrm{C}$ & 0.019506 & -3.663005 & 1.249661 \\
\hline $\mathrm{C}$ & 0.043838 & -5.053701 & 1.221814 \\
\hline $\mathrm{C}$ & 0.069091 & -5.732370 & -0.000947 \\
\hline $\mathrm{C}$ & 0.070527 & -5.004742 & -1.198469 \\
\hline $\mathrm{C}$ & 0.049240 & -3.618612 & -1.169263 \\
\hline $\mathrm{C}$ & -0.039007 & 3.611484 & 1.315849 \\
\hline $\mathrm{C}$ & -0.063283 & 5.002496 & 1.309904 \\
\hline $\mathrm{C}$ & -0.084934 & 5.700534 & 0.098067 \\
\hline $\mathrm{C}$ & -0.082808 & 4.992025 & -1.110843 \\
\hline $\mathrm{C}$ & -0.061454 & 3.605641 & -1.103537 \\
\hline $\mathrm{C}$ & -5.923257 & 1.085651 & 1.235028 \\
\hline $\mathrm{C}$ & -7.089676 & 1.827476 & 1.361846 \\
\hline $\mathrm{C}$ & -7.316502 & 2.935190 & 0.537084 \\
\hline $\mathrm{C}$ & -6.351259 & 3.297366 & -0.411701 \\
\hline $\mathrm{C}$ & -5.185537 & 2.558506 & -0.535241 \\
\hline $\mathrm{C}$ & -5.135873 & -2.690406 & 0.779198 \\
\hline $\mathrm{C}$ & -6.283123 & -3.462840 & 0.641067 \\
\hline
\end{tabular}




\begin{tabular}{|c|c|c|c|}
\hline $\mathrm{C}$ & -7.233699 & -3.145181 & -0.334400 \\
\hline $\mathrm{C}$ & -7.020465 & -2.040916 & -1.172107 \\
\hline $\mathrm{C}$ & -5.882100 & -1.267025 & -1.028165 \\
\hline $\mathrm{C}$ & -8.566291 & 3.709247 & 0.670780 \\
\hline $\mathrm{O}$ & -8.849327 & 4.685436 & 0.005592 \\
\hline $\mathrm{C}$ & -8.447020 & -3.973406 & -0.476315 \\
\hline $\mathrm{O}$ & -9.319535 & -3.785921 & -1.300361 \\
\hline $\mathrm{C}$ & 0.097096 & -7.209854 & -0.021196 \\
\hline $\mathrm{O}$ & 0.114783 & -7.885392 & -1.029676 \\
\hline $\mathrm{C}$ & -0.113267 & 7.178253 & 0.101192 \\
\hline $\mathrm{O}$ & -0.124440 & 7.869551 & -0.896555 \\
\hline $\mathrm{C}$ & 8.424586 & 3.941092 & -0.576169 \\
\hline $\mathrm{O}$ & 9.280735 & 3.762884 & -1.419211 \\
\hline $\mathrm{C}$ & 8.522071 & -3.800869 & 0.485128 \\
\hline $\mathrm{O}$ & 9.399866 & -3.614993 & 1.303951 \\
\hline $\mathrm{H}$ & 2.499768 & -2.461558 & 0.135130 \\
\hline $\mathrm{H}$ & 2.445648 & 2.481505 & -0.003097 \\
\hline $\mathrm{H}$ & -2.461503 & -2.512581 & -0.009767 \\
\hline $\mathrm{H}$ & -2.514247 & 2.426260 & 0.230452 \\
\hline $\mathrm{H}$ & 4.424757 & -2.813604 & -1.443665 \\
\hline $\mathrm{H}$ & 6.497564 & -4.153167 & -1.256469 \\
\hline $\mathrm{H}$ & 7.820146 & -1.669930 & 1.981640 \\
\hline $\mathrm{H}$ & 5.749753 & -0.318262 & 1.792222 \\
\hline $\mathrm{H}$ & 4.422122 & 2.879420 & 1.507069 \\
\hline $\mathrm{H}$ & 6.457631 & 4.262067 & 1.236136 \\
\hline $\mathrm{H}$ & 7.708557 & 1.793107 & -2.041130 \\
\hline $\mathrm{H}$ & 5.675719 & 0.400730 & -1.769872 \\
\hline $\mathrm{H}$ & 0.001316 & -3.136232 & 2.196772 \\
\hline $\mathrm{H}$ & 0.043000 & -5.616711 & 2.149892 \\
\hline $\mathrm{H}$ & 0.089000 & -5.542716 & -2.139108 \\
\hline $\mathrm{H}$ & 0.050438 & -3.053730 & -2.094481 \\
\hline $\mathrm{H}$ & -0.023516 & 3.069883 & 2.254636 \\
\hline $\mathrm{H}$ & -0.065069 & 5.550455 & 2.246851 \\
\hline $\mathrm{H}$ & -0.099202 & 5.544844 & -2.042866 \\
\hline $\mathrm{H}$ & -0.060191 & 3.055361 & -2.037515 \\
\hline $\mathrm{H}$ & -5.756241 & 0.230427 & 1.877928 \\
\hline $\mathrm{H}$ & -7.831503 & 1.548221 & 2.103278 \\
\hline $\mathrm{H}$ & -6.537066 & 4.155125 & -1.047514 \\
\hline $\mathrm{H}$ & -4.448353 & 2.831200 & -1.281503 \\
\hline $\mathrm{H}$ & -4.407089 & -2.933418 & 1.543630 \\
\hline $\mathrm{H}$ & -6.446116 & -4.314757 & 1.293860 \\
\hline $\mathrm{H}$ & -7.759284 & -1.807578 & -1.929829 \\
\hline $\mathrm{H}$ & -5.723634 & -0.415414 & -1.678274 \\
\hline $\mathrm{H}$ & -9.264273 & 3.332785 & 1.446733 \\
\hline $\mathrm{H}$ & -8.520864 & -4.815691 & 0.242130 \\
\hline $\mathrm{H}$ & 0.102006 & -7.687228 & 0.979855 \\
\hline $\mathrm{H}$ & -0.125299 & 7.639634 & 1.109627 \\
\hline $\mathrm{H}$ & 8.513341 & 4.774395 & 0.150884 \\
\hline $\mathrm{H}$ & 8.603666 & -4.625302 & -0.252829 \\
\hline
\end{tabular}


Table S9 Atom coordinates and absolute energies for $\mathbf{2 d}\left(\mathbf{C F}_{3}\right)$ Standard orientation

\begin{tabular}{|c|c|c|c|}
\hline \multirow{2}{*}{ Atomic Type } & \multicolumn{3}{|c|}{ Coordinates (Angstroms) } \\
\hline & $\mathbf{X}$ & $\mathbf{Y}$ & $\mathbf{Z}$ \\
\hline $\mathrm{C}$ & -3.670408 & 0.719475 & 0.034935 \\
\hline $\mathrm{C}$ & -3.672593 & -0.723787 & -0.021781 \\
\hline $\mathrm{C}$ & -2.474524 & -1.395007 & -0.017384 \\
\hline $\mathrm{C}$ & -1.214826 & -0.726668 & 0.011229 \\
\hline $\mathrm{C}$ & -1.212697 & 0.713398 & 0.018952 \\
\hline $\mathrm{C}$ & -2.469985 & 1.386316 & 0.040115 \\
\hline $\mathrm{C}$ & 0.002615 & -1.433412 & 0.015267 \\
\hline $\mathrm{C}$ & 1.222249 & -0.730743 & 0.019714 \\
\hline $\mathrm{C}$ & 1.223987 & 0.709313 & 0.016398 \\
\hline $\mathrm{C}$ & 0.006729 & 1.416054 & 0.019279 \\
\hline $\mathrm{C}$ & 2.480075 & -1.402986 & 0.041303 \\
\hline $\mathrm{C}$ & 3.680320 & -0.735317 & 0.039158 \\
\hline $\mathrm{C}$ & 3.681516 & 0.707861 & -0.013551 \\
\hline $\mathrm{C}$ & 2.483164 & 1.378320 & -0.008094 \\
\hline $\mathrm{C}$ & 0.010671 & 2.909486 & 0.020469 \\
\hline $\mathrm{C}$ & -0.001284 & -2.926836 & 0.013214 \\
\hline $\mathrm{C}$ & 4.937536 & 1.497532 & -0.124504 \\
\hline $\mathrm{C}$ & 4.938527 & -1.522960 & 0.135144 \\
\hline $\mathrm{C}$ & -4.927482 & 1.509139 & 0.131447 \\
\hline $\mathrm{C}$ & -4.931458 & -1.509094 & -0.130223 \\
\hline $\mathrm{C}$ & -5.169118 & -2.587281 & 0.731760 \\
\hline $\mathrm{C}$ & -6.338558 & -3.333974 & 0.634491 \\
\hline $\mathrm{C}$ & -7.286541 & -3.008098 & -0.333119 \\
\hline $\mathrm{C}$ & -7.059098 & -1.946186 & -1.210561 \\
\hline $\mathrm{C}$ & -5.892304 & -1.202500 & -1.105268 \\
\hline $\mathrm{C}$ & -5.152767 & 2.588427 & -0.732580 \\
\hline $\mathrm{C}$ & -6.320466 & 3.339137 & -0.646873 \\
\hline $\mathrm{C}$ & -7.279331 & 3.016176 & 0.310968 \\
\hline $\mathrm{C}$ & -7.064491 & 1.953164 & 1.190168 \\
\hline $\mathrm{C}$ & -5.899133 & 1.205552 & 1.096558 \\
\hline $\mathrm{C}$ & 0.003279 & -3.633759 & -1.194070 \\
\hline $\mathrm{C}$ & -0.010634 & -5.024552 & -1.199722 \\
\hline $\mathrm{C}$ & -0.029840 & -5.718828 & 0.009330 \\
\hline $\mathrm{C}$ & -0.029062 & -5.027669 & 1.220419 \\
\hline $\mathrm{C}$ & -0.016967 & -3.636907 & 1.218620 \\
\hline $\mathrm{C}$ & 0.006337 & 3.618739 & -1.185501 \\
\hline $\mathrm{C}$ & 0.020491 & 5.009487 & -1.188491 \\
\hline $\mathrm{C}$ & 0.039567 & 5.701459 & 0.021940 \\
\hline $\mathrm{C}$ & 0.038472 & 5.008009 & 1.231651 \\
\hline $\mathrm{C}$ & 0.026266 & 3.617209 & 1.227193 \\
\hline $\mathrm{C}$ & 5.890269 & 1.204632 & -1.109589 \\
\hline $\mathrm{C}$ & 7.046567 & 1.965469 & -1.229468 \\
\hline $\mathrm{C}$ & 7.270267 & 3.027163 & -0.353652 \\
\hline $\mathrm{C}$ & 6.331876 & 3.334431 & 0.631900 \\
\hline $\mathrm{C}$ & 5.174315 & 2.574255 & 0.741721 \\
\hline $\mathrm{C}$ & 5.164400 & -2.604224 & -0.726283 \\
\hline
\end{tabular}




\begin{tabular}{|c|c|c|c|}
\hline $\mathrm{C}$ & 6.333047 & -3.353369 & -0.639552 \\
\hline $\mathrm{C}$ & 7.292408 & -3.026749 & 0.316547 \\
\hline $\mathrm{C}$ & 7.077367 & -1.961317 & 1.192762 \\
\hline $\mathrm{C}$ & 5.911088 & -1.215322 & 1.098135 \\
\hline $\mathrm{C}$ & 8.493527 & 3.883556 & -0.490609 \\
\hline $\mathrm{F}$ & 9.477242 & 3.280934 & -1.194641 \\
\hline $\mathrm{F}$ & 9.017925 & 4.223064 & 0.712033 \\
\hline $\mathrm{F}$ & 8.224596 & 5.053677 & -1.127626 \\
\hline $\mathrm{C}$ & 8.543960 & -3.843361 & 0.443279 \\
\hline $\mathrm{F}$ & 8.854278 & -4.498568 & -0.698415 \\
\hline $\mathrm{F}$ & 8.441302 & -4.789716 & 1.412902 \\
\hline $\mathrm{F}$ & 9.618244 & -3.085419 & 0.768851 \\
\hline $\mathrm{C}$ & 0.006118 & -7.218771 & 0.008301 \\
\hline $\mathrm{F}$ & -0.581609 & -7.747945 & -1.089163 \\
\hline $\mathrm{F}$ & -0.615622 & -7.749655 & 1.086339 \\
\hline $\mathrm{F}$ & 1.278238 & -7.694962 & 0.027821 \\
\hline $\mathrm{C}$ & 0.003832 & 7.201410 & 0.022945 \\
\hline $\mathrm{F}$ & 0.601146 & 7.731931 & -1.068762 \\
\hline $\mathrm{F}$ & 0.616213 & 7.730783 & 1.106954 \\
\hline $\mathrm{F}$ & -1.268356 & 7.677766 & 0.031831 \\
\hline $\mathrm{C}$ & -8.530115 & 3.834244 & 0.435917 \\
\hline $\mathrm{F}$ & -8.841595 & 4.484520 & -0.708330 \\
\hline $\mathrm{F}$ & -8.425322 & 4.784966 & 1.401018 \\
\hline $\mathrm{F}$ & -9.604434 & 3.078590 & 0.766422 \\
\hline $\mathrm{C}$ & -8.539190 & -3.821228 & -0.470917 \\
\hline $\mathrm{F}$ & -8.860194 & -4.477636 & 0.667067 \\
\hline $\mathrm{F}$ & -8.431567 & -4.766051 & -1.441556 \\
\hline $\mathrm{F}$ & -9.608863 & -3.059785 & -0.803466 \\
\hline $\mathrm{H}$ & -2.481300 & -2.475586 & -0.077459 \\
\hline $\mathrm{H}$ & -2.472829 & 2.466938 & 0.100268 \\
\hline $\mathrm{H}$ & 2.483441 & -2.483770 & 0.098808 \\
\hline $\mathrm{H}$ & 2.489423 & 2.459040 & -0.065272 \\
\hline $\mathrm{H}$ & -4.441928 & -2.828772 & 1.497912 \\
\hline $\mathrm{H}$ & -6.517809 & -4.155394 & 1.316606 \\
\hline $\mathrm{H}$ & -7.796361 & -1.697319 & -1.963885 \\
\hline $\mathrm{H}$ & -5.723969 & -0.375423 & -1.783521 \\
\hline $\mathrm{H}$ & -4.417094 & 2.827783 & -1.491253 \\
\hline $\mathrm{H}$ & -6.489885 & 4.161566 & -1.330296 \\
\hline $\mathrm{H}$ & -7.810371 & 1.706411 & 1.935639 \\
\hline $\mathrm{H}$ & -5.740519 & 0.377631 & 1.776129 \\
\hline $\mathrm{H}$ & 0.011259 & -3.091031 & -2.132092 \\
\hline $\mathrm{H}$ & -0.014322 & -5.566516 & -2.137186 \\
\hline $\mathrm{H}$ & -0.045091 & -5.572143 & 2.156339 \\
\hline $\mathrm{H}$ & -0.021104 & -3.096629 & 2.158075 \\
\hline $\mathrm{H}$ & -0.001574 & 3.077776 & -2.124542 \\
\hline $\mathrm{H}$ & 0.024540 & 5.553271 & -2.124925 \\
\hline $\mathrm{H}$ & 0.054316 & 5.550675 & 2.168597 \\
\hline $\mathrm{H}$ & 0.030228 & 3.075171 & 2.165635 \\
\hline $\mathrm{H}$ & 5.723557 & 0.378251 & -1.789171 \\
\hline $\mathrm{H}$ & 7.773812 & 1.731659 & -1.996361 \\
\hline
\end{tabular}




\begin{tabular}{cccc}
\hline $\mathrm{H}$ & 6.511169 & 4.154884 & 1.316081 \\
$\mathrm{H}$ & 4.453295 & 2.803893 & 1.517282 \\
$\mathrm{H}$ & 4.428294 & -2.846610 & -1.483568 \\
$\mathrm{H}$ & 6.502731 & -4.177545 & -1.320792 \\
$\mathrm{H}$ & 7.823600 & -1.711722 & 1.936950 \\
$\mathrm{H}$ & 5.752054 & -0.385978 & 1.775850 \\
\hline
\end{tabular}


Table S10 Atom coordinates and absolute energies for 3a Standard orientation

\begin{tabular}{|c|c|c|c|}
\hline \multirow{2}{*}{ Atomic Type } & \multicolumn{3}{|c|}{ Coordinates (Angstroms) } \\
\hline & $\mathbf{X}$ & Y & $\mathbf{Z}$ \\
\hline $\mathrm{C}$ & -6.872154 & 3.041670 & -1.168493 \\
\hline $\mathrm{C}$ & -7.022936 & 1.962661 & -0.289537 \\
\hline $\mathrm{H}$ & -8.000024 & 1.700292 & 0.094119 \\
\hline $\mathrm{C}$ & -5.933569 & 1.194964 & 0.115669 \\
\hline $\mathrm{H}$ & -6.092782 & 0.365200 & 0.793987 \\
\hline $\mathrm{C}$ & -4.641601 & 1.476435 & -0.337995 \\
\hline $\mathrm{C}$ & -4.479774 & 2.566157 & -1.204387 \\
\hline $\mathrm{H}$ & -3.490609 & 2.810271 & -1.577006 \\
\hline $\mathrm{C}$ & -5.570639 & 3.326509 & -1.609208 \\
\hline $\mathrm{H}$ & -5.400516 & 4.155204 & -2.287136 \\
\hline $\mathrm{C}$ & -6.592740 & -3.148910 & -1.671784 \\
\hline $\mathrm{C}$ & -6.036718 & -2.068939 & -2.367595 \\
\hline $\mathrm{H}$ & -6.386504 & -1.817914 & -3.360225 \\
\hline $\mathrm{C}$ & -5.027093 & -1.286127 & -1.811990 \\
\hline $\mathrm{H}$ & -4.627184 & -0.454284 & -2.379777 \\
\hline $\mathrm{C}$ & -4.525617 & -1.552751 & -0.534653 \\
\hline $\mathrm{C}$ & -5.066089 & -2.642247 & 0.162195 \\
\hline $\mathrm{H}$ & -4.704252 & -2.871668 & 1.158818 \\
\hline $\mathrm{C}$ & -6.076789 & -3.416737 & -0.394502 \\
\hline $\mathrm{H}$ & -6.473791 & -4.242203 & 0.184764 \\
\hline $\mathrm{C}$ & 0.026638 & -5.627742 & 2.082138 \\
\hline $\mathrm{C}$ & -0.007911 & -4.816218 & 3.225036 \\
\hline $\mathrm{H}$ & -0.033416 & -5.271153 & 4.208330 \\
\hline $\mathrm{C}$ & -0.011187 & -3.426954 & 3.143597 \\
\hline $\mathrm{H}$ & -0.036658 & -2.839362 & 4.051644 \\
\hline $\mathrm{C}$ & 0.018040 & -2.793178 & 1.900081 \\
\hline $\mathrm{C}$ & 0.048191 & -3.588271 & 0.752018 \\
\hline $\mathrm{H}$ & 0.067953 & -3.120607 & -0.225739 \\
\hline $\mathrm{C}$ & 0.052504 & -4.978119 & 0.842662 \\
\hline $\mathrm{H}$ & 0.075893 & -5.548025 & -0.076535 \\
\hline $\mathrm{C}$ & 0.011254 & -1.287711 & 1.751031 \\
\hline $\mathrm{C}$ & -1.232553 & -0.709273 & 1.088125 \\
\hline $\mathrm{C}$ & -1.247557 & 0.690621 & 1.093971 \\
\hline $\mathrm{C}$ & -2.362049 & 1.369026 & 0.628806 \\
\hline $\mathrm{H}$ & -2.407113 & 2.448091 & 0.692155 \\
\hline $\mathrm{C}$ & -3.466689 & 0.676961 & 0.104984 \\
\hline $\mathrm{C}$ & -3.435293 & -0.734301 & 0.063430 \\
\hline $\mathrm{C}$ & -2.319023 & -1.407380 & 0.587759 \\
\hline $\mathrm{H}$ & -2.309180 & -2.489035 & 0.571812 \\
\hline $\mathrm{O}$ & 0.001037 & -0.731670 & 3.124207 \\
\hline $\mathrm{C}$ & 6.872152 & -3.041672 & -1.168493 \\
\hline $\mathrm{C}$ & 7.022935 & -1.962663 & -0.289537 \\
\hline $\mathrm{H}$ & 8.000022 & -1.700295 & 0.094119 \\
\hline $\mathrm{C}$ & 5.933568 & -1.194966 & 0.115668 \\
\hline $\mathrm{H}$ & 6.092780 & -0.365201 & 0.793986 \\
\hline $\mathrm{C}$ & 4.641600 & -1.476436 & -0.337997 \\
\hline
\end{tabular}




\begin{tabular}{|c|c|c|c|}
\hline $\mathrm{C}$ & 4.479773 & -2.566158 & -1.204390 \\
\hline $\mathrm{H}$ & 3.490607 & -2.810270 & -1.577009 \\
\hline $\mathrm{C}$ & 5.570637 & -3.326511 & -1.609209 \\
\hline $\mathrm{H}$ & 5.400514 & -4.155205 & -2.287137 \\
\hline $\mathrm{C}$ & 6.592741 & 3.148909 & -1.671786 \\
\hline $\mathrm{C}$ & 6.036710 & 2.068946 & -2.367602 \\
\hline $\mathrm{H}$ & 6.386489 & 1.817927 & -3.360236 \\
\hline $\mathrm{C}$ & 5.027085 & 1.286135 & -1.811997 \\
\hline $\mathrm{H}$ & 4.627169 & 0.454297 & -2.379788 \\
\hline $\mathrm{C}$ & 4.525617 & 1.552751 & -0.534656 \\
\hline $\mathrm{C}$ & 5.066097 & 2.642241 & 0.162197 \\
\hline $\mathrm{H}$ & 4.704265 & 2.871657 & 1.158823 \\
\hline $\mathrm{C}$ & 6.076798 & 3.416730 & -0.394500 \\
\hline $\mathrm{H}$ & 6.473806 & 4.242190 & 0.184770 \\
\hline $\mathrm{C}$ & -0.026637 & 5.627744 & 2.082134 \\
\hline $\mathrm{C}$ & 0.007913 & 4.816222 & 3.225032 \\
\hline $\mathrm{H}$ & 0.033419 & 5.271158 & 4.208326 \\
\hline $\mathrm{C}$ & 0.011189 & 3.426957 & 3.143595 \\
\hline $\mathrm{H}$ & 0.036661 & 2.839366 & 4.051642 \\
\hline $\mathrm{C}$ & -0.018039 & 2.793180 & 1.900079 \\
\hline $\mathrm{C}$ & -0.048192 & 3.588272 & 0.752016 \\
\hline $\mathrm{H}$ & -0.067955 & 3.120607 & -0.225741 \\
\hline $\mathrm{C}$ & -0.052505 & 4.978120 & 0.842658 \\
\hline $\mathrm{H}$ & -0.075895 & 5.548026 & -0.076540 \\
\hline $\mathrm{C}$ & -0.011253 & 1.287713 & 1.751031 \\
\hline $\mathrm{C}$ & 1.232553 & 0.709274 & 1.088124 \\
\hline $\mathrm{C}$ & 1.247557 & -0.690619 & 1.093970 \\
\hline $\mathrm{C}$ & 2.362048 & -1.369025 & 0.628804 \\
\hline $\mathrm{H}$ & 2.407112 & -2.448090 & 0.692154 \\
\hline $\mathrm{C}$ & 3.466688 & -0.676961 & 0.104981 \\
\hline $\mathrm{C}$ & 3.435292 & 0.734302 & 0.063428 \\
\hline $\mathrm{C}$ & 2.319024 & 1.407381 & 0.587757 \\
\hline $\mathrm{H}$ & 2.309181 & 2.489036 & 0.571811 \\
\hline $\mathrm{O}$ & -0.001035 & 0.731673 & 3.124207 \\
\hline $\mathrm{C}$ & 0.020453 & -7.158390 & 2.223735 \\
\hline $\mathrm{C}$ & -1.303139 & -7.598549 & 2.888582 \\
\hline $\mathrm{C}$ & 1.207962 & -7.602921 & 3.106813 \\
\hline $\mathrm{C}$ & 0.137695 & -7.871609 & 0.865698 \\
\hline $\mathrm{H}$ & -1.424686 & -7.155239 & 3.879939 \\
\hline $\mathrm{H}$ & -2.162497 & -7.302010 & 2.279780 \\
\hline $\mathrm{H}$ & -1.326657 & -8.686977 & 3.003198 \\
\hline $\mathrm{H}$ & 1.214674 & -8.692672 & 3.207960 \\
\hline $\mathrm{H}$ & 2.160762 & -7.296873 & 2.665216 \\
\hline $\mathrm{H}$ & 1.151136 & -7.176961 & 4.111346 \\
\hline $\mathrm{H}$ & -0.704012 & -7.636317 & 0.208276 \\
\hline $\mathrm{H}$ & 1.063704 & -7.608081 & 0.346458 \\
\hline $\mathrm{H}$ & 0.141030 & -8.954159 & 1.020296 \\
\hline $\mathrm{C}$ & -7.721507 & -4.024386 & -2.240554 \\
\hline $\mathrm{C}$ & -8.155729 & -3.581946 & -3.648347 \\
\hline $\mathrm{C}$ & -8.950729 & -3.938549 & -1.307453 \\
\hline
\end{tabular}




\begin{tabular}{|c|c|c|c|}
\hline $\mathrm{C}$ & -7.243909 & -5.491809 & -2.319074 \\
\hline $\mathrm{H}$ & -8.534895 & -2.556039 & -3.653279 \\
\hline $\mathrm{H}$ & -7.334374 & -3.646764 & -4.367684 \\
\hline $\mathrm{H}$ & -8.959528 & -4.232093 & -4.005177 \\
\hline $\mathrm{H}$ & -9.769698 & -4.548427 & -1.702058 \\
\hline $\mathrm{H}$ & -8.722825 & -4.298763 & -0.301314 \\
\hline $\mathrm{H}$ & -9.304394 & -2.906732 & -1.222738 \\
\hline $\mathrm{H}$ & -6.375227 & -5.585623 & -2.977455 \\
\hline $\mathrm{H}$ & -6.963852 & -5.879878 & -1.336705 \\
\hline $\mathrm{H}$ & -8.040763 & -6.129180 & -2.715531 \\
\hline $\mathrm{C}$ & -0.020453 & 7.158392 & 2.223729 \\
\hline $\mathrm{C}$ & 1.303140 & 7.598553 & 2.888574 \\
\hline $\mathrm{C}$ & -1.207960 & 7.602924 & 3.106809 \\
\hline $\mathrm{C}$ & -0.137696 & 7.871610 & 0.865692 \\
\hline $\mathrm{H}$ & 1.424689 & 7.155243 & 3.879931 \\
\hline $\mathrm{H}$ & 2.162498 & 7.302013 & 2.279771 \\
\hline $\mathrm{H}$ & 1.326659 & 8.686981 & 3.003189 \\
\hline $\mathrm{H}$ & -1.214673 & 8.692675 & 3.207954 \\
\hline $\mathrm{H}$ & -2.160760 & 7.296875 & 2.665213 \\
\hline $\mathrm{H}$ & -1.151133 & 7.176965 & 4.111342 \\
\hline $\mathrm{H}$ & 0.704009 & 7.636317 & 0.208269 \\
\hline $\mathrm{H}$ & -1.063707 & 7.608081 & 0.346453 \\
\hline $\mathrm{H}$ & -0.141032 & 8.954160 & 1.020288 \\
\hline $\mathrm{C}$ & 7.721509 & 4.024385 & -2.240555 \\
\hline $\mathrm{C}$ & 8.155693 & 3.581978 & -3.648370 \\
\hline $\mathrm{C}$ & 8.950750 & 3.938504 & -1.307484 \\
\hline $\mathrm{C}$ & 7.243930 & 5.491817 & -2.319023 \\
\hline $\mathrm{H}$ & 8.534839 & 2.556064 & -3.653341 \\
\hline $\mathrm{H}$ & 7.334325 & 3.646834 & -4.367689 \\
\hline $\mathrm{H}$ & 8.959498 & 4.232120 & -4.005197 \\
\hline $\mathrm{H}$ & 9.769720 & 4.548380 & -1.702092 \\
\hline $\mathrm{H}$ & 8.722875 & 4.298693 & -0.301330 \\
\hline $\mathrm{H}$ & 9.304403 & 2.906679 & -1.222806 \\
\hline $\mathrm{H}$ & 6.375237 & 5.585662 & -2.977385 \\
\hline $\mathrm{H}$ & 6.963896 & 5.879860 & -1.336637 \\
\hline $\mathrm{H}$ & 8.040785 & 6.129189 & -2.715475 \\
\hline $\mathrm{C}$ & 8.050063 & -3.904054 & -1.650076 \\
\hline $\mathrm{C}$ & 8.122774 & -3.857205 & -3.193056 \\
\hline $\mathrm{C}$ & 7.833761 & -5.364017 & -1.191838 \\
\hline $\mathrm{C}$ & 9.398222 & -3.417300 & -1.091965 \\
\hline $\mathrm{H}$ & 7.214414 & -4.249847 & -3.656510 \\
\hline $\mathrm{H}$ & 8.262609 & -2.830884 & -3.545088 \\
\hline $\mathrm{H}$ & 8.965474 & -4.457143 & -3.550878 \\
\hline $\mathrm{H}$ & 8.658224 & -5.997420 & -1.534874 \\
\hline $\mathrm{H}$ & 7.789108 & -5.427228 & -0.100610 \\
\hline $\mathrm{H}$ & 6.905047 & -5.780225 & -1.590014 \\
\hline $\mathrm{H}$ & 9.618343 & -2.391734 & -1.401688 \\
\hline $\mathrm{H}$ & 9.425056 & -3.458601 & 0.000479 \\
\hline $\mathrm{H}$ & 10.202740 & -4.056302 & -1.466831 \\
\hline $\mathrm{C}$ & -8.050065 & 3.904050 & -1.650077 \\
\hline
\end{tabular}




\begin{tabular}{llll}
\hline $\mathrm{C}$ & -8.122775 & 3.857200 & -3.193058 \\
$\mathrm{C}$ & -7.833765 & 5.364014 & -1.191841 \\
$\mathrm{C}$ & -9.398224 & 3.417295 & -1.091968 \\
$\mathrm{H}$ & -7.214415 & 4.249843 & -3.656511 \\
$\mathrm{H}$ & -8.262609 & 2.830878 & -3.545089 \\
$\mathrm{H}$ & -8.965475 & 4.457137 & -3.550881 \\
$\mathrm{H}$ & -8.658228 & 5.997415 & -1.534877 \\
$\mathrm{H}$ & -7.789113 & 5.427225 & -0.100612 \\
$\mathrm{H}$ & -6.905051 & 5.780222 & -1.590015 \\
$\mathrm{H}$ & -9.618344 & 2.391728 & -1.401690 \\
$\mathrm{H}$ & -9.425060 & 3.458597 & 0.000477 \\
$\mathrm{H}$ & -10.202743 & 4.056296 & -1.466834 \\
\hline
\end{tabular}


Table S11 Atom coordinates and absolute energies for 3b Standard orientation

\begin{tabular}{|c|c|c|c|}
\hline \multirow{2}{*}{ Atomic Type } & \multicolumn{3}{|c|}{ Coordinates (Angstroms) } \\
\hline & $\mathbf{X}$ & $\mathbf{Y}$ & $\mathbf{Z}$ \\
\hline $\mathrm{C}$ & -3.468174 & 0.662301 & -0.050342 \\
\hline $\mathrm{C}$ & -3.426371 & -0.749867 & -0.029975 \\
\hline $\mathrm{C}$ & -2.309503 & -1.389802 & 0.534459 \\
\hline $\mathrm{C}$ & -1.231888 & -0.663744 & 1.012670 \\
\hline $\mathrm{C}$ & -1.255874 & 0.735049 & 0.956119 \\
\hline $\mathrm{C}$ & -2.371330 & 1.383573 & 0.452187 \\
\hline $\mathrm{C}$ & 0.013458 & -1.205560 & 1.703279 \\
\hline $\mathrm{C}$ & 1.245179 & -0.627048 & 1.021055 \\
\hline $\mathrm{C}$ & 1.222418 & 0.771587 & 0.956744 \\
\hline $\mathrm{C}$ & -0.026784 & 1.369611 & 1.592449 \\
\hline $\mathrm{C}$ & 2.361349 & -1.318239 & 0.580155 \\
\hline $\mathrm{C}$ & 3.459351 & -0.644421 & 0.019346 \\
\hline $\mathrm{C}$ & 3.421072 & 0.764557 & -0.080039 \\
\hline $\mathrm{C}$ & 2.304543 & 1.453238 & 0.424672 \\
\hline $\mathrm{C}$ & -0.048021 & 2.879289 & 1.681048 \\
\hline $\mathrm{C}$ & 0.031523 & -2.703154 & 1.922074 \\
\hline $\mathrm{C}$ & 4.503721 & 1.559793 & -0.721630 \\
\hline $\mathrm{C}$ & 4.630603 & -1.457187 & -0.408299 \\
\hline $\mathrm{C}$ & -4.643321 & 1.434405 & -0.538259 \\
\hline $\mathrm{C}$ & -4.496574 & -1.607441 & -0.609057 \\
\hline $\mathrm{C}$ & -5.016028 & -2.692313 & 0.116472 \\
\hline $\mathrm{C}$ & -5.987639 & -3.520914 & -0.424108 \\
\hline $\mathrm{C}$ & -6.468969 & -3.292619 & -1.719339 \\
\hline $\mathrm{C}$ & -5.960785 & -2.220353 & -2.460339 \\
\hline $\mathrm{C}$ & -4.987595 & -1.394559 & -1.900886 \\
\hline $\mathrm{C}$ & -4.477676 & 2.496353 & -1.442086 \\
\hline $\mathrm{C}$ & -5.561222 & 3.238043 & -1.888838 \\
\hline $\mathrm{C}$ & -6.853042 & 2.941736 & -1.436655 \\
\hline $\mathrm{C}$ & -7.036806 & 1.897905 & -0.523387 \\
\hline $\mathrm{C}$ & -5.938680 & 1.158623 & -0.089604 \\
\hline $\mathrm{C}$ & 0.014101 & -3.277296 & 3.197877 \\
\hline $\mathrm{C}$ & 0.025299 & -4.656612 & 3.354548 \\
\hline $\mathrm{C}$ & 0.053226 & -5.499923 & 2.237720 \\
\hline $\mathrm{C}$ & 0.071336 & -4.938135 & 0.956449 \\
\hline $\mathrm{C}$ & 0.060879 & -3.552339 & 0.813553 \\
\hline $\mathrm{C}$ & -0.071263 & 3.628756 & 0.494328 \\
\hline $\mathrm{C}$ & -0.091472 & 5.012814 & 0.524323 \\
\hline $\mathrm{C}$ & -0.089344 & 5.691603 & 1.751042 \\
\hline $\mathrm{C}$ & -0.065220 & 4.957527 & 2.938857 \\
\hline $\mathrm{C}$ & -0.044524 & 3.561422 & 2.894583 \\
\hline $\mathrm{C}$ & 5.056269 & 2.675459 & -0.072109 \\
\hline $\mathrm{C}$ & 6.058162 & 3.429972 & -0.664661 \\
\hline $\mathrm{C}$ & 6.536265 & 3.092702 & -1.937210 \\
\hline $\mathrm{C}$ & 5.988253 & 1.992922 & -2.605961 \\
\hline $\mathrm{C}$ & 4.986701 & 1.242139 & -1.994630 \\
\hline $\mathrm{C}$ & 4.457570 & -2.579779 & -1.233999 \\
\hline $\mathrm{C}$ & 5.536962 & -3.354804 & -1.631548 \\
\hline
\end{tabular}




\begin{tabular}{|c|c|c|c|}
\hline $\mathrm{C}$ & 6.831581 & -3.031677 & -1.206657 \\
\hline $\mathrm{C}$ & 7.022880 & -1.925993 & -0.371086 \\
\hline $\mathrm{C}$ & 5.928561 & -1.154707 & 0.014656 \\
\hline $\mathrm{O}$ & -0.002999 & -0.590175 & 3.051303 \\
\hline $\mathrm{O}$ & -0.016908 & 0.871366 & 2.988053 \\
\hline $\mathrm{O}$ & 7.524654 & 3.887961 & -2.432597 \\
\hline $\mathrm{C}$ & 8.065830 & 3.580336 & -3.717397 \\
\hline $\mathrm{O}$ & 7.825325 & -3.846927 & -1.656531 \\
\hline $\mathrm{C}$ & 9.169984 & -3.557471 & -1.274065 \\
\hline $\mathrm{O}$ & 0.059837 & -6.835450 & 2.497773 \\
\hline $\mathrm{C}$ & 0.085488 & -7.742452 & 1.394804 \\
\hline $\mathrm{O}$ & -0.111570 & 7.049480 & 1.673836 \\
\hline $\mathrm{C}$ & -0.113796 & 7.803548 & 2.886804 \\
\hline $\mathrm{O}$ & -7.851937 & 3.718817 & -1.939253 \\
\hline $\mathrm{C}$ & -9.194936 & 3.445731 & -1.539165 \\
\hline $\mathrm{O}$ & -7.419023 & -4.162466 & -2.161125 \\
\hline $\mathrm{C}$ & -7.946413 & -3.988175 & -3.476163 \\
\hline $\mathrm{H}$ & -2.290600 & -2.471028 & 0.563912 \\
\hline $\mathrm{H}$ & -2.424205 & 2.464220 & 0.468488 \\
\hline $\mathrm{H}$ & 2.412128 & -2.393300 & 0.690804 \\
\hline $\mathrm{H}$ & 2.290123 & 2.533458 & 0.365681 \\
\hline $\mathrm{H}$ & -4.663619 & -2.878953 & 1.124955 \\
\hline $\mathrm{H}$ & -6.393841 & -4.351651 & 0.141133 \\
\hline $\mathrm{H}$ & -6.308714 & -2.020923 & -3.464904 \\
\hline $\mathrm{H}$ & -4.603392 & -0.568822 & -2.487647 \\
\hline $\mathrm{H}$ & -3.485888 & 2.733703 & -1.811250 \\
\hline $\mathrm{H}$ & -5.432498 & 4.049737 & -2.595376 \\
\hline $\mathrm{H}$ & -8.020123 & 1.653142 & -0.145354 \\
\hline $\mathrm{H}$ & -6.097960 & 0.352567 & 0.616272 \\
\hline $\mathrm{H}$ & -0.008483 & -2.645544 & 4.075429 \\
\hline $\mathrm{H}$ & 0.012115 & -5.104249 & 4.341335 \\
\hline $\mathrm{H}$ & 0.092266 & -5.558443 & 0.071026 \\
\hline $\mathrm{H}$ & 0.073880 & -3.132155 & -0.185280 \\
\hline $\mathrm{H}$ & -0.073196 & 3.119724 & -0.462484 \\
\hline $\mathrm{H}$ & -0.109180 & 5.592310 & -0.391063 \\
\hline $\mathrm{H}$ & -0.062097 & 5.451486 & 3.900846 \\
\hline $\mathrm{H}$ & -0.026485 & 3.010241 & 3.825178 \\
\hline $\mathrm{H}$ & 4.707616 & 2.942804 & 0.919524 \\
\hline $\mathrm{H}$ & 6.491997 & 4.283602 & -0.156859 \\
\hline $\mathrm{H}$ & 6.329561 & 1.712866 & -3.593100 \\
\hline $\mathrm{H}$ & 4.576928 & 0.389659 & -2.522803 \\
\hline $\mathrm{H}$ & 3.463549 & -2.838604 & -1.582117 \\
\hline $\mathrm{H}$ & 5.402816 & -4.214224 & -2.278117 \\
\hline $\mathrm{H}$ & 8.008764 & -1.656852 & -0.016991 \\
\hline $\mathrm{H}$ & 6.093850 & -0.299292 & 0.658459 \\
\hline $\mathrm{H}$ & 8.839108 & 4.325767 & -3.900103 \\
\hline $\mathrm{H}$ & 8.516981 & 2.582094 & -3.734408 \\
\hline $\mathrm{H}$ & 7.304486 & 3.651575 & -4.501977 \\
\hline $\mathrm{H}$ & 9.784311 & -4.319910 & -1.751863 \\
\hline $\mathrm{H}$ & 9.484005 & -2.568780 & -1.626798 \\
\hline
\end{tabular}




\begin{tabular}{lccc}
\hline $\mathrm{H}$ & 9.301684 & -3.618568 & -0.188327 \\
$\mathrm{H}$ & 0.084041 & -8.740413 & 1.831422 \\
$\mathrm{H}$ & -0.799723 & -7.625658 & 0.760420 \\
$\mathrm{H}$ & 0.991228 & -7.614382 & 0.792482 \\
$\mathrm{H}$ & -0.132505 & 8.850041 & 2.584712 \\
$\mathrm{H}$ & -1.000582 & 7.586871 & 3.492372 \\
$\mathrm{H}$ & 0.789236 & 7.615243 & 3.477629 \\
$\mathrm{H}$ & -9.815047 & 4.163640 & -2.074941 \\
$\mathrm{H}$ & -9.498039 & 2.429919 & -1.816117 \\
$\mathrm{H}$ & -9.330759 & 3.588667 & -0.461619 \\
$\mathrm{H}$ & -8.669442 & -4.791372 & -3.614275 \\
$\mathrm{H}$ & -8.455500 & -3.023670 & -3.580654 \\
$\mathrm{H}$ & -7.163699 & -4.073857 & -4.238017 \\
\hline
\end{tabular}


Table S12 Atom coordinates and absolute energies for 3c Standard orientation

\begin{tabular}{|c|c|c|c|}
\hline \multirow{2}{*}{ Atomic Type } & \multicolumn{3}{|c|}{ Coordinates (Angstroms) } \\
\hline & $\mathbf{X}$ & $\mathbf{Y}$ & $\mathbf{Z}$ \\
\hline $\mathrm{C}$ & -3.457958 & 0.638745 & -0.095590 \\
\hline $\mathrm{C}$ & -3.406617 & -0.771487 & -0.071273 \\
\hline $\mathrm{C}$ & -2.286713 & -1.406195 & 0.490013 \\
\hline $\mathrm{C}$ & -1.214913 & -0.668975 & 0.964716 \\
\hline $\mathrm{C}$ & -1.250121 & 0.729694 & 0.907183 \\
\hline $\mathrm{C}$ & -2.368412 & 1.371566 & 0.402138 \\
\hline $\mathrm{C}$ & 0.032417 & -1.195893 & 1.663508 \\
\hline $\mathrm{C}$ & 1.266078 & -0.611187 & 0.989591 \\
\hline $\mathrm{C}$ & 1.231380 & 0.787074 & 0.922582 \\
\hline $\mathrm{C}$ & -0.028392 & 1.372408 & 1.549244 \\
\hline $\mathrm{C}$ & 2.392286 & -1.295378 & 0.563627 \\
\hline $\mathrm{C}$ & 3.490472 & -0.608597 & 0.022045 \\
\hline $\mathrm{C}$ & 3.442391 & 0.798291 & -0.076784 \\
\hline $\mathrm{C}$ & 2.313423 & 1.479881 & 0.406181 \\
\hline $\mathrm{C}$ & -0.060481 & 2.883089 & 1.633715 \\
\hline $\mathrm{C}$ & 0.063138 & -2.693555 & 1.881869 \\
\hline $\mathrm{C}$ & 4.525420 & 1.602496 & -0.705050 \\
\hline $\mathrm{C}$ & 4.681839 & -1.405828 & -0.375214 \\
\hline $\mathrm{C}$ & -4.646234 & 1.400016 & -0.566869 \\
\hline $\mathrm{C}$ & -4.472694 & -1.630324 & -0.653935 \\
\hline $\mathrm{C}$ & -4.996970 & -2.703197 & 0.080638 \\
\hline $\mathrm{C}$ & -5.974690 & -3.523401 & -0.470003 \\
\hline $\mathrm{C}$ & -6.440525 & -3.291377 & -1.767945 \\
\hline $\mathrm{C}$ & -5.912019 & -2.226914 & -2.511633 \\
\hline $\mathrm{C}$ & -4.942483 & -1.406870 & -1.960419 \\
\hline $\mathrm{C}$ & -4.491714 & 2.462144 & -1.468758 \\
\hline $\mathrm{C}$ & -5.592696 & 3.202818 & -1.882488 \\
\hline $\mathrm{C}$ & -6.868624 & 2.899823 & -1.396970 \\
\hline $\mathrm{C}$ & -7.028518 & 1.843519 & -0.489088 \\
\hline $\mathrm{C}$ & -5.932470 & 1.102435 & -0.082403 \\
\hline $\mathrm{C}$ & 0.046816 & -3.252566 & 3.160666 \\
\hline $\mathrm{C}$ & 0.074714 & -4.636380 & 3.314222 \\
\hline $\mathrm{C}$ & 0.118171 & -5.472905 & 2.197002 \\
\hline $\mathrm{C}$ & 0.133916 & -4.911599 & 0.913021 \\
\hline $\mathrm{C}$ & 0.107060 & -3.536142 & 0.758168 \\
\hline $\mathrm{C}$ & -0.082161 & 3.619888 & 0.442323 \\
\hline $\mathrm{C}$ & -0.107647 & 5.007970 & 0.476878 \\
\hline $\mathrm{C}$ & -0.111647 & 5.679473 & 1.704276 \\
\hline $\mathrm{C}$ & -0.090893 & 4.943105 & 2.893699 \\
\hline $\mathrm{C}$ & -0.064632 & 3.555485 & 2.861232 \\
\hline $\mathrm{C}$ & 5.045782 & 2.722964 & -0.042433 \\
\hline $\mathrm{C}$ & 6.039287 & 3.493759 & -0.634940 \\
\hline $\mathrm{C}$ & 6.525932 & 3.162437 & -1.903483 \\
\hline $\mathrm{C}$ & 6.003759 & 2.047402 & -2.574329 \\
\hline $\mathrm{C}$ & 5.018234 & 1.277132 & -1.981361 \\
\hline $\mathrm{C}$ & 5.958775 & -1.082400 & 0.104647 \\
\hline $\mathrm{C}$ & 7.059948 & -1.847161 & -0.256282 \\
\hline
\end{tabular}




\begin{tabular}{|c|c|c|c|}
\hline $\mathrm{C}$ & 6.908206 & -2.947745 & -1.107218 \\
\hline $\mathrm{C}$ & 5.633812 & -3.280708 & -1.584350 \\
\hline $\mathrm{C}$ & 4.534222 & -2.520540 & -1.218644 \\
\hline $\mathrm{O}$ & 0.001664 & -0.580893 & 3.008195 \\
\hline $\mathrm{O}$ & -0.022528 & 0.878397 & 2.943351 \\
\hline $\mathrm{C}$ & 7.578807 & 3.989806 & -2.525183 \\
\hline $\mathrm{O}$ & 8.060554 & 3.786636 & -3.621408 \\
\hline $\mathrm{C}$ & 8.087797 & -3.750025 & -1.489026 \\
\hline $\mathrm{O}$ & 8.057156 & -4.718911 & -2.220674 \\
\hline $\mathrm{C}$ & 0.149402 & -6.940550 & 2.374309 \\
\hline $\mathrm{O}$ & 0.190751 & -7.741365 & 1.463244 \\
\hline $\mathrm{C}$ & -0.139537 & 7.157619 & 1.734192 \\
\hline $\mathrm{O}$ & -0.137222 & 7.826037 & 2.747012 \\
\hline $\mathrm{C}$ & -8.031380 & 3.697598 & -1.835136 \\
\hline $\mathrm{O}$ & -9.173490 & 3.514812 & -1.465129 \\
\hline $\mathrm{C}$ & -7.480717 & -4.167686 & -2.342211 \\
\hline $\mathrm{O}$ & -7.939429 & -4.054131 & -3.460970 \\
\hline $\mathrm{H}$ & -2.263023 & -2.487140 & 0.519818 \\
\hline $\mathrm{H}$ & -2.431015 & 2.451336 & 0.416358 \\
\hline $\mathrm{H}$ & 2.452755 & -2.369962 & 0.671477 \\
\hline $\mathrm{H}$ & 2.291462 & 2.559386 & 0.344143 \\
\hline $\mathrm{H}$ & -4.649596 & -2.880163 & 1.091978 \\
\hline $\mathrm{H}$ & -6.383414 & -4.345367 & 0.109131 \\
\hline $\mathrm{H}$ & -6.273748 & -2.060778 & -3.519570 \\
\hline $\mathrm{H}$ & -4.535668 & -0.587347 & -2.539958 \\
\hline $\mathrm{H}$ & -3.506490 & 2.696296 & -1.855395 \\
\hline $\mathrm{H}$ & -5.465417 & 4.018199 & -2.587582 \\
\hline $\mathrm{H}$ & -8.020098 & 1.622097 & -0.112019 \\
\hline $\mathrm{H}$ & -6.061848 & 0.292251 & 0.624403 \\
\hline $\mathrm{H}$ & 0.013573 & -2.614138 & 4.032311 \\
\hline $\mathrm{H}$ & 0.063424 & -5.067615 & 4.309994 \\
\hline $\mathrm{H}$ & 0.168162 & -5.568385 & 0.051919 \\
\hline $\mathrm{H}$ & 0.120130 & -3.105668 & -0.235899 \\
\hline $\mathrm{H}$ & -0.078007 & 3.102257 & -0.509437 \\
\hline $\mathrm{H}$ & -0.123154 & 5.573639 & -0.449138 \\
\hline $\mathrm{H}$ & -0.095492 & 5.473068 & 3.838854 \\
\hline $\mathrm{H}$ & -0.048468 & 2.995706 & 3.786034 \\
\hline $\mathrm{H}$ & 4.681271 & 2.976246 & 0.946516 \\
\hline $\mathrm{H}$ & 6.444309 & 4.354084 & -0.111453 \\
\hline $\mathrm{H}$ & 6.382504 & 1.802953 & -3.559896 \\
\hline $\mathrm{H}$ & 4.618100 & 0.417253 & -2.504162 \\
\hline $\mathrm{H}$ & 6.082918 & -0.232439 & 0.764228 \\
\hline $\mathrm{H}$ & 8.044754 & -1.592262 & 0.122126 \\
\hline $\mathrm{H}$ & 5.526462 & -4.134703 & -2.242946 \\
\hline $\mathrm{H}$ & 3.551142 & -2.775170 & -1.597934 \\
\hline $\mathrm{H}$ & 7.919762 & 4.846608 & -1.908307 \\
\hline $\mathrm{H}$ & 9.045825 & -3.402512 & -1.050801 \\
\hline $\mathrm{H}$ & 0.132644 & -7.282064 & 3.429094 \\
\hline $\mathrm{H}$ & -0.164237 & 7.641310 & 0.736491 \\
\hline $\mathrm{H}$ & -7.792725 & 4.510428 & -2.551613 \\
\hline
\end{tabular}


Table S13 Atom coordinates and absolute energies for 3d Standard orientation

\begin{tabular}{|c|c|c|c|}
\hline \multirow{2}{*}{ Atomic Type } & \multicolumn{3}{|c|}{ Coordinates (Angstroms) } \\
\hline & $\mathbf{X}$ & $\mathbf{Y}$ & $\mathbf{Z}$ \\
\hline $\mathrm{C}$ & -6.879163 & 2.933550 & -1.116272 \\
\hline $\mathrm{C}$ & -7.043987 & 1.864357 & -0.235159 \\
\hline $\mathrm{H}$ & -8.028265 & 1.621674 & 0.145502 \\
\hline $\mathrm{C}$ & -5.943139 & 1.109639 & 0.147046 \\
\hline $\mathrm{H}$ & -6.075751 & 0.280306 & 0.830426 \\
\hline $\mathrm{C}$ & -4.662697 & 1.413362 & -0.336042 \\
\hline $\mathrm{C}$ & -4.513770 & 2.496664 & -1.212084 \\
\hline $\mathrm{H}$ & -3.532289 & 2.739872 & -1.602150 \\
\hline $\mathrm{C}$ & -5.612755 & 3.253195 & -1.602771 \\
\hline $\mathrm{H}$ & -5.488248 & 4.080966 & -2.289609 \\
\hline $\mathrm{C}$ & -6.496187 & -3.196434 & -1.698835 \\
\hline $\mathrm{C}$ & -5.962432 & -2.124017 & -2.414313 \\
\hline $\mathrm{H}$ & -6.321086 & -1.904552 & -3.412211 \\
\hline $\mathrm{C}$ & -4.975132 & -1.335175 & -1.839550 \\
\hline $\mathrm{H}$ & -4.566714 & -0.501163 & -2.396261 \\
\hline $\mathrm{C}$ & -4.502085 & -1.606153 & -0.548112 \\
\hline $\mathrm{C}$ & -5.040536 & -2.694033 & 0.151878 \\
\hline $\mathrm{H}$ & -4.694042 & -2.909922 & 1.155885 \\
\hline $\mathrm{C}$ & -6.032640 & -3.485298 & -0.416265 \\
\hline $\mathrm{H}$ & -6.452656 & -4.314569 & 0.139020 \\
\hline $\mathrm{C}$ & 0.082140 & -5.573635 & 2.093942 \\
\hline $\mathrm{C}$ & 0.059961 & -4.795651 & 3.247309 \\
\hline $\mathrm{H}$ & 0.048513 & -5.267856 & 4.221099 \\
\hline $\mathrm{C}$ & 0.044071 & -3.406184 & 3.149559 \\
\hline $\mathrm{H}$ & 0.021337 & -2.809770 & 4.050840 \\
\hline $\mathrm{C}$ & 0.055111 & -2.790017 & 1.898245 \\
\hline $\mathrm{C}$ & 0.082930 & -3.582851 & 0.742946 \\
\hline $\mathrm{H}$ & 0.090164 & -3.114246 & -0.233615 \\
\hline $\mathrm{C}$ & 0.096900 & -4.967277 & 0.836474 \\
\hline $\mathrm{H}$ & 0.111306 & -5.572475 & -0.061622 \\
\hline $\mathrm{C}$ & 0.028129 & -1.284664 & 1.744494 \\
\hline $\mathrm{C}$ & -1.224958 & -0.725991 & 1.081591 \\
\hline $\mathrm{C}$ & -1.257516 & 0.673737 & 1.085377 \\
\hline $\mathrm{C}$ & -2.379172 & 1.339710 & 0.620357 \\
\hline $\mathrm{H}$ & -2.439689 & 2.417958 & 0.681813 \\
\hline $\mathrm{C}$ & -3.473422 & 0.631183 & 0.099477 \\
\hline $\mathrm{C}$ & -3.424819 & -0.778013 & 0.060195 \\
\hline $\mathrm{C}$ & -2.302589 & -1.439578 & 0.584064 \\
\hline $\mathrm{H}$ & -2.282759 & -2.520974 & 0.568623 \\
\hline $\mathrm{O}$ & 0.012486 & -0.728062 & 3.114725 \\
\hline $\mathrm{C}$ & 6.871679 & -2.938135 & -1.129945 \\
\hline $\mathrm{C}$ & 7.038966 & -1.867428 & -0.251095 \\
\hline $\mathrm{H}$ & 8.024296 & -1.624155 & 0.126459 \\
\hline $\mathrm{C}$ & 5.939212 & -1.111928 & 0.132733 \\
\hline $\mathrm{H}$ & 6.073730 & -0.281248 & 0.814107 \\
\hline $\mathrm{C}$ & 4.657473 & -1.416453 & -0.346377 \\
\hline $\mathrm{C}$ & 4.506095 & -2.501186 & -1.220215 \\
\hline
\end{tabular}




\begin{tabular}{|c|c|c|c|}
\hline $\mathrm{H}$ & 3.523542 & -2.744939 & -1.607222 \\
\hline $\mathrm{C}$ & 5.603967 & -3.258489 & -1.612570 \\
\hline $\mathrm{H}$ & 5.477502 & -4.087434 & -2.297636 \\
\hline $\mathrm{C}$ & 6.462853 & 3.219189 & -1.725535 \\
\hline $\mathrm{C}$ & 5.950706 & 2.132057 & -2.431279 \\
\hline $\mathrm{H}$ & 6.306918 & 1.915104 & -3.430126 \\
\hline $\mathrm{C}$ & 4.972411 & 1.333296 & -1.850908 \\
\hline $\mathrm{H}$ & 4.572904 & 0.493085 & -2.404767 \\
\hline $\mathrm{C}$ & 4.494614 & 1.605718 & -0.562694 \\
\hline $\mathrm{C}$ & 5.018267 & 2.706237 & 0.131397 \\
\hline $\mathrm{H}$ & 4.665427 & 2.926222 & 1.132242 \\
\hline $\mathrm{C}$ & 5.997127 & 3.507288 & -0.441971 \\
\hline $\mathrm{H}$ & 6.396625 & 4.353986 & 0.103235 \\
\hline $\mathrm{C}$ & -0.079516 & 5.575508 & 2.074967 \\
\hline $\mathrm{C}$ & -0.055436 & 4.801822 & 3.231182 \\
\hline $\mathrm{H}$ & -0.041709 & 5.277691 & 4.203145 \\
\hline $\mathrm{C}$ & -0.040420 & 3.411958 & 3.138659 \\
\hline $\mathrm{H}$ & -0.015993 & 2.818890 & 4.042118 \\
\hline $\mathrm{C}$ & -0.054333 & 2.791191 & 1.889696 \\
\hline $\mathrm{C}$ & -0.084406 & 3.579703 & 0.731505 \\
\hline $\mathrm{H}$ & -0.094097 & 3.107240 & -0.243179 \\
\hline $\mathrm{C}$ & -0.097341 & 4.964457 & 0.819801 \\
\hline $\mathrm{H}$ & -0.113351 & 5.566250 & -0.080558 \\
\hline $\mathrm{C}$ & -0.027882 & 1.285384 & 1.741017 \\
\hline $\mathrm{C}$ & 1.223102 & 0.724837 & 1.076178 \\
\hline $\mathrm{C}$ & 1.256034 & -0.674818 & 1.083561 \\
\hline $\mathrm{C}$ & 2.376739 & -1.341167 & 0.616542 \\
\hline $\mathrm{H}$ & 2.438001 & -2.419259 & 0.680146 \\
\hline $\mathrm{C}$ & 3.469395 & -0.633417 & 0.090806 \\
\hline $\mathrm{C}$ & 3.420381 & 0.775685 & 0.048419 \\
\hline $\mathrm{C}$ & 2.298735 & 1.437420 & 0.573564 \\
\hline $\mathrm{H}$ & 2.277668 & 2.518664 & 0.555360 \\
\hline $\mathrm{O}$ & -0.008284 & 0.732965 & 3.112767 \\
\hline $\mathrm{C}$ & -7.530188 & -4.075708 & -2.337085 \\
\hline $\mathrm{F}$ & -8.318665 & -3.398822 & -3.204007 \\
\hline $\mathrm{F}$ & -8.347201 & -4.655884 & -1.428235 \\
\hline $\mathrm{F}$ & -6.967003 & -5.089231 & -3.046265 \\
\hline $\mathrm{C}$ & -8.058443 & 3.783257 & -1.486651 \\
\hline $\mathrm{F}$ & -7.922947 & 4.350611 & -2.707893 \\
\hline $\mathrm{F}$ & -8.242820 & 4.805178 & -0.610150 \\
\hline $\mathrm{F}$ & -9.215435 & 3.081926 & -1.501157 \\
\hline $\mathrm{C}$ & -0.137941 & 7.072910 & 2.162445 \\
\hline $\mathrm{F}$ & -1.400481 & 7.539612 & 1.985602 \\
\hline $\mathrm{F}$ & 0.622194 & 7.669302 & 1.213121 \\
\hline $\mathrm{F}$ & 0.282259 & 7.540694 & 3.358075 \\
\hline $\mathrm{C}$ & 7.544298 & 4.077105 & -2.311402 \\
\hline $\mathrm{F}$ & 8.751097 & 3.834504 & -1.736664 \\
\hline $\mathrm{F}$ & 7.300181 & 5.397950 & -2.127503 \\
\hline $\mathrm{F}$ & 7.704228 & 3.886808 & -3.639268 \\
\hline $\mathrm{C}$ & 8.049875 & -3.788630 & -1.501966 \\
\hline
\end{tabular}




\begin{tabular}{lccc}
\hline F & 8.236144 & -4.809376 & -0.624536 \\
$F$ & 9.206961 & -3.087528 & -1.520287 \\
$F$ & 7.911329 & -4.357653 & -2.722109 \\
C & 0.141950 & -7.070641 & 2.187090 \\
$F$ & -0.618444 & -7.671393 & 1.240735 \\
$F$ & -0.276832 & -7.534127 & 3.384896 \\
$F$ & 1.404748 & -7.536933 & 2.011032 \\
\hline
\end{tabular}


Table S14Atom coordinates and absolute energies for $\mathbf{4 b}$ Standard orientation

\begin{tabular}{|c|c|c|c|}
\hline \multirow{2}{*}{ Atomic Type } & \multicolumn{3}{|c|}{ Coordinates (Angstroms) } \\
\hline & $\mathbf{X}$ & Y & $\mathbf{X}$ \\
\hline $\mathrm{O}$ & 0.013350 & 3.015836 & 0.885734 \\
\hline $\mathrm{O}$ & 0.008197 & -1.906841 & -1.360841 \\
\hline $\mathrm{H}$ & 0.009873 & -1.333764 & -2.138396 \\
\hline $\mathrm{O}$ & -8.337432 & 4.486793 & -0.138369 \\
\hline $\mathrm{O}$ & 8.433750 & -3.073216 & -1.513694 \\
\hline $\mathrm{O}$ & -8.421086 & -3.045064 & -1.538544 \\
\hline $\mathrm{O}$ & 8.366302 & 4.459191 & -0.135581 \\
\hline $\mathrm{C}$ & -1.254161 & 1.144856 & 0.171460 \\
\hline $\mathrm{C}$ & 1.277389 & -0.212506 & -0.187912 \\
\hline $\mathrm{C}$ & 0.011313 & 1.864082 & 0.466486 \\
\hline $\mathrm{C}$ & 1.274677 & 1.138635 & 0.176765 \\
\hline $\mathrm{C}$ & -4.936192 & -0.870488 & -0.772336 \\
\hline $\mathrm{C}$ & -2.462729 & 1.849717 & 0.213599 \\
\hline $\mathrm{H}$ & -2.426731 & 2.901377 & 0.470342 \\
\hline $\mathrm{C}$ & 4.951455 & -0.893199 & -0.751462 \\
\hline $\mathrm{C}$ & -2.477223 & -0.811385 & -0.502198 \\
\hline $\mathrm{H}$ & -2.479335 & -1.864757 & -0.751678 \\
\hline $\mathrm{C}$ & 2.491751 & -0.825166 & -0.485601 \\
\hline $\mathrm{H}$ & 2.490340 & -1.879103 & -0.732772 \\
\hline $\mathrm{C}$ & -1.261505 & -0.205061 & -0.197311 \\
\hline $\mathrm{C}$ & 3.708734 & -0.136991 & -0.445447 \\
\hline $\mathrm{C}$ & -5.005564 & -1.693390 & -1.900376 \\
\hline $\mathrm{H}$ & -4.157000 & -1.748038 & -2.573506 \\
\hline $\mathrm{C}$ & 2.485913 & 1.838558 & 0.221098 \\
\hline $\mathrm{H}$ & 2.453382 & 2.891099 & 0.474640 \\
\hline $\mathrm{C}$ & 3.704576 & 1.239082 & -0.089594 \\
\hline $\mathrm{C}$ & -3.682993 & 1.256060 & -0.102038 \\
\hline $\mathrm{C}$ & -4.908963 & 2.099689 & -0.087901 \\
\hline $\mathrm{C}$ & 0.013732 & -1.435896 & 2.314637 \\
\hline $\mathrm{H}$ & 0.046323 & -0.361036 & 2.449892 \\
\hline $\mathrm{C}$ & 6.076852 & -0.839036 & 0.089355 \\
\hline $\mathrm{H}$ & 6.055321 & -0.214218 & 0.973518 \\
\hline $\mathrm{C}$ & -0.007605 & -1.982383 & 1.023591 \\
\hline $\mathrm{C}$ & -3.691556 & -0.118703 & -0.463004 \\
\hline $\mathrm{C}$ & 0.005692 & -1.057657 & -0.206195 \\
\hline $\mathrm{C}$ & 7.270318 & -2.393322 & -1.327330 \\
\hline $\mathrm{C}$ & 4.933163 & 2.079031 & -0.077268 \\
\hline $\mathrm{O}$ & -0.077691 & -4.357527 & 4.452461 \\
\hline $\mathrm{C}$ & 7.214857 & -1.576162 & -0.188963 \\
\hline $\mathrm{H}$ & 8.078249 & -1.539878 & 0.464904 \\
\hline $\mathrm{C}$ & 5.019753 & -1.718391 & -1.877887 \\
\hline $\mathrm{H}$ & 4.171287 & -1.772954 & -2.551140 \\
\hline $\mathrm{C}$ & -7.204155 & 3.737621 & -0.054072 \\
\hline $\mathrm{C}$ & -6.149303 & -2.432871 & -2.201076 \\
\hline $\mathrm{H}$ & -6.162729 & -3.046822 & -3.091081 \\
\hline $\mathrm{C}$ & 6.162581 & -2.459842 & -2.177160 \\
\hline $\mathrm{H}$ & 6.175323 & -3.075387 & -3.066072 \\
\hline $\mathrm{C}$ & 6.923782 & 2.936497 & -1.175457 \\
\hline $\mathrm{H}$ & 7.586888 & 2.980634 & -2.031526 \\
\hline $\mathrm{C}$ & 5.796631 & 2.132692 & -1.184441 \\
\hline
\end{tabular}




\begin{tabular}{|c|c|c|c|}
\hline $\mathrm{H}$ & 5.577538 & 1.537506 & -2.062792 \\
\hline $\mathrm{C}$ & -5.772112 & 2.159022 & -1.195037 \\
\hline $\mathrm{H}$ & -5.554101 & 1.566455 & -2.075417 \\
\hline $\mathrm{C}$ & -6.897650 & 2.965054 & -1.183439 \\
\hline $\mathrm{H}$ & -7.560544 & 3.013503 & -2.039439 \\
\hline $\mathrm{C}$ & -6.355546 & 3.696474 & 1.055512 \\
\hline $\mathrm{H}$ & -6.564656 & 4.280115 & 1.941806 \\
\hline $\mathrm{C}$ & -5.221251 & 2.883878 & 1.025274 \\
\hline $\mathrm{H}$ & -4.576402 & 2.852301 & 1.896768 \\
\hline $\mathrm{C}$ & 6.382779 & 3.676828 & 1.060831 \\
\hline $\mathrm{H}$ & 6.592790 & 4.263294 & 1.945046 \\
\hline $\mathrm{C}$ & -7.256992 & -2.366565 & -1.351169 \\
\hline $\mathrm{C}$ & 5.246820 & 2.866450 & 1.033205 \\
\hline $\mathrm{H}$ & 4.601571 & 2.839484 & 1.904557 \\
\hline $\mathrm{C}$ & -6.061766 & -0.816103 & 0.068252 \\
\hline $\mathrm{H}$ & -6.039462 & -0.193040 & 0.953633 \\
\hline $\mathrm{C}$ & -0.052531 & -3.366475 & 0.890908 \\
\hline $\mathrm{H}$ & -0.070866 & -3.808424 & -0.095798 \\
\hline $\mathrm{C}$ & -0.055408 & -3.645165 & 3.292047 \\
\hline $\mathrm{C}$ & -0.008782 & -2.250476 & 3.433244 \\
\hline $\mathrm{H}$ & 0.006651 & -1.830790 & 4.432206 \\
\hline $\mathrm{C}$ & 7.231609 & 3.712456 & -0.048776 \\
\hline $\mathrm{C}$ & -0.075843 & -4.201343 & 2.012304 \\
\hline $\mathrm{H}$ & -0.110355 & -5.272978 & 1.869423 \\
\hline $\mathrm{C}$ & -7.200688 & -1.551278 & -0.211503 \\
\hline $\mathrm{H}$ & -8.064086 & -1.515051 & 0.442363 \\
\hline $\mathrm{C}$ & -8.707668 & 5.291151 & 0.981405 \\
\hline $\mathrm{H}$ & -9.630440 & 5.793067 & 0.692530 \\
\hline $\mathrm{H}$ & -7.944083 & 6.043686 & 1.205987 \\
\hline $\mathrm{H}$ & -8.893643 & 4.679356 & 1.870910 \\
\hline $\mathrm{C}$ & -8.532488 & -3.909072 & -2.670481 \\
\hline $\mathrm{H}$ & -9.526946 & -4.349424 & -2.608691 \\
\hline $\mathrm{H}$ & -7.781963 & -4.706498 & -2.640926 \\
\hline $\mathrm{H}$ & -8.442246 & -3.353308 & -3.610242 \\
\hline $\mathrm{C}$ & 8.544836 & -3.937876 & -2.645166 \\
\hline $\mathrm{H}$ & 7.793749 & -4.734759 & -2.615462 \\
\hline $\mathrm{H}$ & 9.538957 & -4.378916 & -2.582884 \\
\hline $\mathrm{H}$ & 8.455254 & -3.382465 & -3.585204 \\
\hline $\mathrm{C}$ & 8.737971 & 5.266833 & 0.981345 \\
\hline $\mathrm{H}$ & 7.976044 & 6.021957 & 1.202860 \\
\hline $\mathrm{H}$ & 9.661961 & 5.765591 & 0.690905 \\
\hline $\mathrm{H}$ & 8.922282 & 4.657955 & 1.873187 \\
\hline $\mathrm{C}$ & -0.154529 & -5.780728 & 4.374581 \\
\hline $\mathrm{H}$ & -1.068564 & -6.105479 & 3.865022 \\
\hline $\mathrm{H}$ & -0.173780 & -6.133120 & 5.405295 \\
\hline $\mathrm{H}$ & 0.719138 & -6.203369 & 3.866113 \\
\hline
\end{tabular}


Table S15 Atom coordinates and absolute energies for $\mathbf{4 b}$ ' Standard orientation

\begin{tabular}{|c|c|c|c|}
\hline \multirow{2}{*}{ Atomic Type } & \multicolumn{3}{|c|}{ Coordinates (Angstroms) } \\
\hline & $\mathbf{X}$ & $\mathbf{Y}$ & $\mathbf{Z}$ \\
\hline $\mathrm{C}$ & -9.534662 & -3.630218 & -1.137564 \\
\hline $\mathrm{H}$ & -10.268217 & -4.393402 & -0.880179 \\
\hline $\mathrm{H}$ & -9.322170 & -3.683423 & -2.210950 \\
\hline $\mathrm{H}$ & -9.940032 & -2.642685 & -0.891717 \\
\hline $\mathrm{C}$ & -7.294331 & -3.106909 & -0.466319 \\
\hline $\mathrm{C}$ & -7.216252 & -2.000528 & -1.318931 \\
\hline $\mathrm{H}$ & -8.041521 & -1.734509 & -1.965333 \\
\hline $\mathrm{C}$ & -6.058904 & -1.225542 & -1.340377 \\
\hline $\mathrm{H}$ & -6.015007 & -0.373047 & -2.007162 \\
\hline $\mathrm{C}$ & -4.959613 & -1.522703 & -0.528467 \\
\hline $\mathrm{C}$ & -5.053927 & -2.643242 & 0.313663 \\
\hline $\mathrm{H}$ & -4.222614 & -2.896259 & 0.962555 \\
\hline $\mathrm{C}$ & -6.199762 & -3.424270 & 0.347722 \\
\hline $\mathrm{H}$ & -6.274054 & -4.281958 & 1.006150 \\
\hline $\mathrm{C}$ & -9.219212 & 3.683151 & 1.372457 \\
\hline $\mathrm{H}$ & -9.985437 & 4.453430 & 1.289679 \\
\hline $\mathrm{H}$ & -8.734263 & 3.763365 & 2.351463 \\
\hline $\mathrm{H}$ & -9.687282 & 2.697317 & 1.274440 \\
\hline $\mathrm{C}$ & -7.221743 & 3.110065 & 0.176573 \\
\hline $\mathrm{C}$ & -6.939793 & 2.022377 & 1.010243 \\
\hline $\mathrm{H}$ & -7.584481 & 1.773381 & 1.842216 \\
\hline $\mathrm{C}$ & -5.808727 & 1.244920 & 0.770472 \\
\hline $\mathrm{H}$ & -5.604220 & 0.405296 & 1.423899 \\
\hline $\mathrm{C}$ & -4.935530 & 1.523196 & -0.285499 \\
\hline $\mathrm{C}$ & -5.230538 & 2.624108 & -1.105348 \\
\hline $\mathrm{H}$ & -4.575532 & 2.860829 & -1.936640 \\
\hline $\mathrm{C}$ & -6.355621 & 3.404628 & -0.883797 \\
\hline $\mathrm{H}$ & -6.587226 & 4.248657 & -1.523097 \\
\hline $\mathrm{C}$ & -0.102549 & 7.299208 & 2.158337 \\
\hline $\mathrm{H}$ & -0.062600 & 7.917737 & 3.054319 \\
\hline $\mathrm{H}$ & -1.061079 & 7.459081 & 1.652422 \\
\hline $\mathrm{H}$ & 0.717567 & 7.581835 & 1.489066 \\
\hline $\mathrm{C}$ & 0.016048 & 4.953648 & 1.683072 \\
\hline $\mathrm{C}$ & -0.120344 & 5.142197 & 0.307304 \\
\hline $\mathrm{H}$ & -0.227356 & 6.132704 & -0.113631 \\
\hline $\mathrm{C}$ & -0.122798 & 4.033492 & -0.546382 \\
\hline $\mathrm{H}$ & -0.241234 & 4.186110 & -1.611507 \\
\hline $\mathrm{C}$ & 0.012890 & 2.740521 & -0.056333 \\
\hline $\mathrm{C}$ & 0.146384 & 2.566166 & 1.329315 \\
\hline $\mathrm{H}$ & 0.252474 & 1.566701 & 1.736761 \\
\hline $\mathrm{C}$ & 0.148838 & 3.650853 & 2.188781 \\
\hline $\mathrm{H}$ & 0.250067 & 3.519419 & 3.259829 \\
\hline $\mathrm{C}$ & 0.000609 & 1.517954 & -0.985840 \\
\hline $\mathrm{C}$ & -1.270991 & 0.702394 & -0.776151 \\
\hline $\mathrm{C}$ & -1.269788 & -0.691843 & -0.784344 \\
\hline $\mathrm{C}$ & -2.490883 & -1.364879 & -0.685235 \\
\hline $\mathrm{H}$ & -2.490768 & -2.448503 & -0.714293 \\
\hline
\end{tabular}




\begin{tabular}{|c|c|c|c|}
\hline $\mathrm{C}$ & -3.716642 & -0.706072 & -0.569957 \\
\hline $\mathrm{C}$ & -3.710957 & 0.709175 & -0.515084 \\
\hline $\mathrm{C}$ & -2.487549 & 1.372420 & -0.632941 \\
\hline $\mathrm{H}$ & -2.481729 & 2.453555 & -0.576259 \\
\hline $\mathrm{O}$ & -8.375815 & -3.927553 & -0.358396 \\
\hline $\mathrm{O}$ & -8.298444 & 3.933729 & 0.310822 \\
\hline $\mathrm{O}$ & 0.031796 & 5.951806 & 2.609802 \\
\hline $\mathrm{O}$ & -0.069109 & 1.926016 & -2.372304 \\
\hline $\mathrm{C}$ & 9.534539 & 3.630326 & -1.138431 \\
\hline $\mathrm{H}$ & 10.268115 & 4.393508 & -0.881101 \\
\hline $\mathrm{H}$ & 9.321926 & 3.683567 & -2.211791 \\
\hline $\mathrm{H}$ & 9.939947 & 2.642789 & -0.892665 \\
\hline $\mathrm{C}$ & 7.294295 & 3.106962 & -0.466939 \\
\hline $\mathrm{C}$ & 7.216133 & 2.000602 & -1.319572 \\
\hline $\mathrm{H}$ & 8.041330 & 1.734616 & -1.966079 \\
\hline $\mathrm{C}$ & 6.058796 & 1.225599 & -1.340904 \\
\hline $\mathrm{H}$ & 6.014835 & 0.373123 & -2.007710 \\
\hline $\mathrm{C}$ & 4.959595 & 1.522718 & -0.528856 \\
\hline $\mathrm{C}$ & 5.053987 & 2.643242 & 0.313286 \\
\hline $\mathrm{H}$ & 4.222744 & 2.896232 & 0.962278 \\
\hline $\mathrm{C}$ & 6.199814 & 3.424286 & 0.347235 \\
\hline $\mathrm{H}$ & 6.274167 & 4.281960 & 1.005675 \\
\hline $\mathrm{C}$ & 9.219132 & -3.683355 & 1.372092 \\
\hline $\mathrm{H}$ & 9.985350 & -4.453637 & 1.289267 \\
\hline $\mathrm{H}$ & 8.734140 & -3.763666 & 2.351068 \\
\hline $\mathrm{H}$ & 9.687223 & -2.697519 & 1.274201 \\
\hline $\mathrm{C}$ & 7.221721 & -3.110121 & 0.176180 \\
\hline $\mathrm{C}$ & 6.939773 & -2.022483 & 1.009917 \\
\hline $\mathrm{H}$ & 7.584454 & -1.773547 & 1.841913 \\
\hline $\mathrm{C}$ & 5.808717 & -1.245000 & 0.770185 \\
\hline $\mathrm{H}$ & 5.604210 & -0.405419 & 1.423667 \\
\hline $\mathrm{C}$ & 4.935523 & -1.523203 & -0.285809 \\
\hline $\mathrm{C}$ & 5.230525 & -2.624071 & -1.105719 \\
\hline $\mathrm{H}$ & 4.575519 & -2.860739 & -1.937026 \\
\hline $\mathrm{C}$ & 6.355604 & -3.404609 & -0.884214 \\
\hline $\mathrm{H}$ & 6.587204 & -4.248603 & -1.523563 \\
\hline $\mathrm{C}$ & 0.102767 & -7.299183 & 2.158383 \\
\hline $\mathrm{H}$ & 0.062865 & -7.917707 & 3.054371 \\
\hline $\mathrm{H}$ & 1.061286 & -7.459031 & 1.652438 \\
\hline $\mathrm{H}$ & -0.717362 & -7.581839 & 1.489140 \\
\hline $\mathrm{C}$ & -0.015910 & -4.953629 & 1.683107 \\
\hline $\mathrm{C}$ & 0.120439 & -5.142184 & 0.307336 \\
\hline $\mathrm{H}$ & 0.227462 & -6.132691 & -0.113596 \\
\hline $\mathrm{C}$ & 0.122833 & -4.033485 & -0.546358 \\
\hline $\mathrm{H}$ & 0.241237 & -4.186108 & -1.611486 \\
\hline $\mathrm{C}$ & -0.012870 & -2.740515 & -0.056314 \\
\hline $\mathrm{C}$ & -0.146317 & -2.566153 & 1.329338 \\
\hline $\mathrm{H}$ & -0.252417 & -1.566687 & 1.736781 \\
\hline $\mathrm{C}$ & -0.148713 & -3.650834 & 2.188812 \\
\hline $\mathrm{H}$ & -0.249907 & -3.519395 & 3.259862 \\
\hline
\end{tabular}




\begin{tabular}{lccc}
\hline $\mathrm{C}$ & -0.000655 & -1.517954 & -0.985832 \\
$\mathrm{C}$ & 1.270960 & -0.702393 & -0.776238 \\
$\mathrm{C}$ & 1.269756 & 0.691843 & -0.784435 \\
$\mathrm{C}$ & 2.490857 & 1.364880 & -0.685414 \\
$\mathrm{H}$ & 2.490738 & 2.448505 & -0.714479 \\
$\mathrm{C}$ & 3.716626 & 0.706076 & -0.570225 \\
$\mathrm{C}$ & 3.710944 & -0.709173 & -0.515340 \\
$\mathrm{C}$ & 2.487528 & -1.372418 & -0.633112 \\
$\mathrm{H}$ & 2.481710 & -2.453552 & -0.576416 \\
$\mathrm{O}$ & 8.375777 & 3.927621 & -0.359121 \\
$\mathrm{O}$ & 8.298408 & -3.933810 & 0.310391 \\
$\mathrm{O}$ & -0.031604 & -5.951781 & 2.609844 \\
$\mathrm{O}$ & 0.068967 & -1.926027 & -2.372298 \\
$\mathrm{H}$ & 0.816098 & 2.207496 & -2.637964 \\
$\mathrm{H}$ & -0.816256 & -2.207527 & -2.637886 \\
\hline
\end{tabular}


Table S16 atom coordinates and absolute energies for $\mathbf{5 b}$ Standard orientation

\begin{tabular}{|c|c|c|c|}
\hline \multirow{2}{*}{ Atomic Type } & \multicolumn{3}{|c|}{ Coordinates (Angstroms) } \\
\hline & $\mathbf{X}$ & Y & $\mathbf{Z}$ \\
\hline $\mathrm{O}$ & -0.057543 & -3.416856 & 0.039877 \\
\hline $\mathrm{O}$ & 8.308096 & -4.515643 & -0.987601 \\
\hline $\mathrm{O}$ & -8.405411 & 3.217287 & 0.157942 \\
\hline $\mathrm{O}$ & 8.415161 & 2.895421 & 1.085310 \\
\hline $\mathrm{O}$ & -8.416184 & -4.562084 & -0.162062 \\
\hline $\mathrm{C}$ & 1.219307 & -1.422467 & -0.016063 \\
\hline $\mathrm{C}$ & -1.272215 & 0.020878 & 0.057416 \\
\hline $\mathrm{C}$ & -0.048197 & -2.194937 & 0.037971 \\
\hline $\mathrm{C}$ & -1.301593 & -1.400382 & 0.098493 \\
\hline $\mathrm{C}$ & 4.926548 & 0.736979 & 0.337584 \\
\hline $\mathrm{C}$ & 2.416142 & -2.106768 & -0.073752 \\
\hline $\mathrm{H}$ & 2.375244 & -3.184983 & -0.161194 \\
\hline $\mathrm{C}$ & -4.970924 & 0.857536 & 0.014031 \\
\hline $\mathrm{C}$ & 2.466333 & 0.639232 & 0.168794 \\
\hline $\mathrm{H}$ & 2.494738 & 1.699214 & 0.374732 \\
\hline $\mathrm{C}$ & -2.513698 & 0.685501 & -0.025602 \\
\hline $\mathrm{H}$ & -2.533172 & 1.762182 & -0.105318 \\
\hline $\mathrm{C}$ & 1.214064 & -0.001494 & 0.038359 \\
\hline $\mathrm{C}$ & -3.739764 & 0.023957 & 0.008632 \\
\hline $\mathrm{C}$ & 5.170613 & 1.917407 & -0.369353 \\
\hline $\mathrm{H}$ & 4.458698 & 2.251353 & -1.116364 \\
\hline $\mathrm{C}$ & -2.510413 & -2.065991 & 0.109687 \\
\hline $\mathrm{H}$ & -2.488052 & -3.148061 & 0.097484 \\
\hline $\mathrm{C}$ & -3.752724 & -1.397458 & 0.072662 \\
\hline $\mathrm{C}$ & 3.666311 & -1.453481 & -0.072669 \\
\hline $\mathrm{C}$ & 4.878913 & -2.259385 & -0.280228 \\
\hline $\mathrm{C}$ & -0.703873 & 2.944232 & 1.012605 \\
\hline $\mathrm{H}$ & -1.250785 & 2.432191 & 1.794438 \\
\hline $\mathrm{C}$ & -5.952320 & 0.698164 & 1.006949 \\
\hline $\mathrm{H}$ & -5.827592 & -0.062289 & 1.768473 \\
\hline $\mathrm{C}$ & 0.002723 & 2.191964 & 0.045213 \\
\hline $\mathrm{C}$ & 3.680074 & -0.041524 & 0.117055 \\
\hline $\mathrm{C}$ & -0.021386 & 0.731795 & 0.050582 \\
\hline $\mathrm{C}$ & -7.266198 & 2.488791 & 0.050234 \\
\hline $\mathrm{C}$ & -4.977637 & -2.214827 & 0.036271 \\
\hline $\mathrm{O}$ & 0.045895 & 6.346784 & 0.101911 \\
\hline $\mathrm{C}$ & -7.077829 & 1.501825 & 1.030268 \\
\hline $\mathrm{H}$ & -7.831814 & 1.386495 & 1.799752 \\
\hline $\mathrm{C}$ & -5.164974 & 1.849362 & -0.950229 \\
\hline $\mathrm{H}$ & -4.428428 & 1.984377 & -1.734877 \\
\hline $\mathrm{C}$ & 7.170788 & -3.841710 & -0.717855 \\
\hline $\mathrm{C}$ & 6.325062 & 2.670675 & -0.157381 \\
\hline $\mathrm{H}$ & 6.484432 & 3.571242 & -0.734764 \\
\hline $\mathrm{C}$ & -6.299862 & 2.659820 & -0.946218 \\
\hline $\mathrm{H}$ & -6.419105 & 3.407180 & -1.718669 \\
\hline $\mathrm{C}$ & -7.184728 & -2.704436 & -0.855899 \\
\hline
\end{tabular}




\begin{tabular}{|c|c|c|c|}
\hline $\mathrm{H}$ & -8.011450 & -2.471397 & -1.515838 \\
\hline $\mathrm{C}$ & -6.065348 & -1.899322 & -0.805198 \\
\hline $\mathrm{H}$ & -6.020075 & -1.024942 & -1.439989 \\
\hline $\mathrm{C}$ & 5.928020 & -1.806828 & -1.108666 \\
\hline $\mathrm{H}$ & 5.851292 & -0.843579 & -1.595538 \\
\hline $\mathrm{C}$ & 7.044590 & -2.584314 & -1.334037 \\
\hline $\mathrm{H}$ & 7.841379 & -2.245558 & -1.985114 \\
\hline $\mathrm{C}$ & 6.142243 & -4.309441 & 0.111450 \\
\hline $\mathrm{H}$ & 6.215684 & -5.269922 & 0.602389 \\
\hline $\mathrm{C}$ & 5.011080 & -3.527400 & 0.308866 \\
\hline $\mathrm{H}$ & 4.231530 & -3.901200 & 0.962550 \\
\hline $\mathrm{C}$ & -6.207523 & -4.181734 & 0.797621 \\
\hline $\mathrm{H}$ & -6.249898 & -5.054406 & 1.434267 \\
\hline $\mathrm{C}$ & 7.260002 & 2.247633 & 0.792851 \\
\hline $\mathrm{C}$ & -5.076114 & -3.375390 & 0.821173 \\
\hline $\mathrm{H}$ & -4.264847 & -3.644220 & 1.487832 \\
\hline $\mathrm{C}$ & 5.876864 & 0.328442 & 1.289630 \\
\hline $\mathrm{H}$ & 5.712504 & -0.578210 & 1.859299 \\
\hline $\mathrm{C}$ & 0.741106 & 2.893365 & -0.928016 \\
\hline $\mathrm{H}$ & 1.262534 & 2.340056 & -1.699352 \\
\hline $\mathrm{C}$ & 0.074713 & 5.006640 & 0.022094 \\
\hline $\mathrm{C}$ & -0.650451 & 4.320950 & 1.014719 \\
\hline $\mathrm{H}$ & -1.156737 & 4.900329 & 1.776951 \\
\hline $\mathrm{C}$ & -7.274436 & -3.851015 & -0.048146 \\
\hline $\mathrm{C}$ & 0.763393 & 4.278511 & -0.961331 \\
\hline $\mathrm{H}$ & 1.308105 & 4.781533 & -1.747720 \\
\hline $\mathrm{C}$ & 7.020301 & 1.070974 & 1.519180 \\
\hline $\mathrm{H}$ & 7.749281 & 0.762044 & 2.258763 \\
\hline $\mathrm{C}$ & 8.509508 & -5.803568 & -0.393099 \\
\hline $\mathrm{H}$ & 9.479163 & -6.143982 & -0.752357 \\
\hline $\mathrm{H}$ & 7.736134 & -6.510814 & -0.708762 \\
\hline $\mathrm{H}$ & 8.529899 & -5.736524 & 0.699142 \\
\hline $\mathrm{C}$ & 8.724202 & 4.098788 & 0.378446 \\
\hline $\mathrm{H}$ & 9.685677 & 4.430763 & 0.767441 \\
\hline $\mathrm{H}$ & 7.972984 & 4.874462 & 0.562298 \\
\hline $\mathrm{H}$ & 8.813948 & 3.916985 & -0.697829 \\
\hline $\mathrm{C}$ & -8.657888 & 4.244225 & -0.804300 \\
\hline $\mathrm{H}$ & -7.875452 & 5.010445 & -0.785764 \\
\hline $\mathrm{H}$ & -9.606999 & 4.692189 & -0.513575 \\
\hline $\mathrm{H}$ & -8.747671 & 3.831543 & -1.814727 \\
\hline $\mathrm{C}$ & -8.574232 & -5.753527 & 0.618039 \\
\hline $\mathrm{H}$ & -7.802456 & -6.490374 & 0.375164 \\
\hline $\mathrm{H}$ & -9.551372 & -6.150943 & 0.348836 \\
\hline $\mathrm{H}$ & -8.554318 & -5.530878 & 1.689506 \\
\hline $\mathrm{C}$ & 0.773745 & 7.125715 & -0.859944 \\
\hline $\mathrm{H}$ & 1.845126 & 6.911503 & -0.808478 \\
\hline $\mathrm{H}$ & 0.596136 & 8.163849 & -0.586328 \\
\hline $\mathrm{H}$ & 0.401696 & 6.947295 & -1.873113 \\
\hline
\end{tabular}


Table S17 Cartesian coordinates of optimized $\mathrm{O}_{2}$.

\begin{tabular}{|c|c|c|c|}
\hline \multirow{2}{*}{ Atomic Type } & \multicolumn{3}{|c|}{ Coordinates (Angstroms) } \\
\hline & $\mathbf{X}$ & $\mathbf{Y}$ & $\mathbf{Z}$ \\
\hline $\mathrm{O}$ & 0.000000 & 0.000000 & 0.603127 \\
\hline $\mathrm{O}$ & 0.000000 & 0.000000 & -0.603127 \\
\hline
\end{tabular}

